Hugo Mann

Theorie und Politik der

Steuerreform in der

Demokratie 
Hugo Mann

\section{Theorie und Politik der Steuerreform in der Demokratie}

Die Vertreter eines konstitutionellen Steuerreformansatzes lehnen die Reformempfehlungen der Theorie der optimalen Besteuerung (OT) mit dem Hinweis auf die mangelhafte Berücksichtigung politisch-ökonomischer Zusammenhänge ab. Sie befürchten, daß die Effizienzregeln der OT in der Hand eines Steuerstaates zur perfekten Ausbeutung der Steuerzahler mißbraucht werden könnten. Vor dem Hintergrund konzeptioneller Defizite der modernen, normativen Steuertheorie werden jene Faktoren untersucht, die bei der Durchsetzung rationaler Steuerreformen von ausschlaggebender Bedeutung sind. Die positive Analyse des demokratischen Steuerreformprozesses belegt, daß eine effiziente und gerechte Besteuerung der Zensiten nur durch eine Steuerverfassung gewährleistet werden kann. Der Autor entwickelt u.a. eine Verfassungsregel, die eine norminkonsistente Vergabe von Steuerprivilegien verhindern soll.

Hugo Mann wurde 1954 in Siegelsbach geboren. Studium der Volkswirtschaftslehre von 1976 bis 1980 an den Universitäten Karlsruhe und Heidelberg. Von 1981 bis 1985 Wissenschaftlicher Mitarbeiter bei Prof. Dr. Cay Folkers am Lehrstuhl für Finanzwissenschaft der Universität Hohenheim in Stuttgart. Seit Januar 1986 im Bereich Konzernrevision der BMW AG tätig. 
Theorie und Politik der Steuerreform in der Demokratie 


\section{Hohenheimer Volkswirtschaftlicheschriften}

Herausgegeben von

Prof.Dr. Cay Folkers

Prof.Dr. Klaus Herdzina

Prof.Dr.Franz Mehler †

Prof.Dr. Walter Piesch

Prof.Dr.Ingoschmidt

Prof.Dr. Helmut Walter

Prof.Dr. Josua Werner

\section{Band 4}

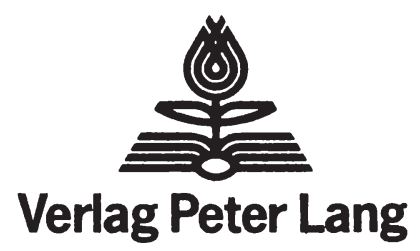

Frankfurt am Main · Bern · New York 


\section{Hugo Mann}

\section{Theorie und Politik \\ der \\ Steuerreform \\ in der Demokratie}

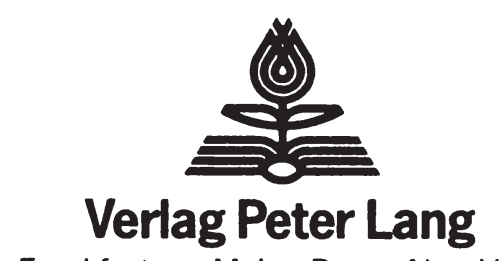

Frankfurt am Main · Bern · New York 
CIP-Kurztitelaufnahme der Deutschen Bibliothek

Mann, Hugo:

Theorie und Politik der Steuerreform in der

Demokratie / Hugo Mann. - Frankfurt am Main ;

Bern ; New York : Lang, 1987.

(Hohenheimer volkswirtschaftliche Schriften ; Bd. 4)

ISBN 3-8204-9464-2

NE: GT

Open Access: The online version of this publication is published on www.peterlang.com and www.econstor.eu under the international Creative Commons License CC-BY 4.0. Learn more on how you can use and share this work: http://creativecommons.org/ licenses/by/4.0.

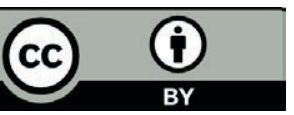

This book is available Open Access thanks to the kind support of ZBW - Leibniz-Informationszentrum Wirtschaft.

\author{
ISSN 0721-3085 \\ ISBN 3-8204-9464-2 \\ ISBN 978-3-631-75532-7 (eBook) \\ (C) Verlag Peter Lang GmbH, Frankfurt am Main 1987 \\ Alle Rechte vorbehalten.
}

Das Werk einschließlich aller seiner Teile ist urheberrechtlich geschützt. Jede Verwertung außerhalb der engen Grenzen des Urheberrechtsgesetzes ist ohne Zustimmung des Verlages unzulässig und strafbar. Das gilt insbesondere für Vervielfältigungen, Übersetzungen, Mikroverfilmungen und die Einspeicherung und Verarbeitung in elektronischen Systemen.

Druck und Bindung: Weihert-Druck GmbH, Darmstadt 
Inhaltsverzeichnis

Seite

I. EINLEITUNG

1. Problemstellung

2. Gang der Untersuchung

4

II. OPTIMALE STEUERREFORMEN UNTER EFFIZIENZASPEKTEN 7

1. Das Konzept der Theorie der optimalen Besteuerung (OT)

2. Die Modellstruktur 13

2.1. Der Konsumsektor 15

2.1.1. Das primale Optimierungsproblem 15

2.1.2. Das duale Optimierungsproblem 16

2.2. Der Produktionssektor 19

2.3. Der Regierungssektor 21

2.4. Die Marktgleichgewichtsbedingungen 23

2.5. Das Walras-Theorem 24

2.6. Die Normierung 25

2.7. Das staatliche Optimierungsproblem 26

3. Steuerregeln für optimale Steuersysteme 27

3.1. First-Best-Lösungen und Pauschalsteuern 28

3.2. Second-Best-Steuerregeln 30

3.2.1. Die Ramsey-Regel 31

3.2.2. Die Corlett-Hague-Regel 32

3.2.3. Proportionale Steuerregeln 34

3.2.4. Die inverse Elastizitätsregel 36

4. Allgemeine Grundsätze optimaler Steverreformen 37

5. Das Problem schrittweiser Steuerreformen 39

5.1. Die Ableitung des Wohlfahrtskriteriums 40

6. Die Interpretation der Steuerreformergebnisse 41

7. Die Relevanz des allgemeinen Gleichgewichtsmodells

8. Zusammenfassung 46 
III. DIE INFORMATIONSPROBLEMATIK OPTIMALER STEUER-

Seite

REFORMEN

1. Die empirische Sensibilität optimaler Steuerregeln

1.1. Zur Spezifikation von Nachfragesystemen

1.1.1. Additive Nutzenfunktionen

1.1.2. Homothetische Nutzenfunktionen 49

1.1.3. Stone-Geary-Funktionen 50

1.2. Weitere Probleme der empirischen Analyse 53

2. Zur finanzpolitischen Relevanz von Ein-Konsumenten-Modellen

IV. OPTIMALE STEUERREFORMEN UNTER VERTEILUNGSASPEKTEN 59

1. OT-Empfehlungen im Mehr-Personen-Modell 60

2. Zur Umverteilungswirkung alternativer sozialer Wohl fahrtsfunktionen

3. Fazit

V. INSTITUTIONELLE ASPEKTE VON STEUERREFORMEN UND KONSTITUTIONELLE STEUERREGELN

1. Alternative Hypothesen über die Verhaltensweise des Regierungssektors im Steuerreformprozeß

1.1. Der Leviathan-Steuerstaat 74

1.2. Das Modell 77

1.3. Die Steuerregeln des Leviathan 80

1.4. Die Konsequenzen der Maximalbesteuerung 84

2. Die Logik fiskalischer Verfassunasregeln 86

3. Konstitutionelle Steuerregeln von Brennan/ Buchanan

3.1. Prozedurale und ergebnisbezogene fiskalische Verfassungsregeln

3.2. Die Modellgrundlage 91

3.2.1. Begrenzungen des Steuerinstrumentariums und der Steuerstruktur

3.2.2. Einheitliche Steuerstrukturen für alle Steuerzahler

3.2.3. Zur Wahl der Steuerbemessungsgrundlage 96 
3.2.4. Die Begrenzung einzelner Steuersätze 100

3.2.5. Begrenzungen des Steueraufkommens 101

3.2.6. Zweckgebundene Steuern 104

4. Zur Kritik an den konstitutionellen Steuer-
regeln

5. Die weltanschauliche Basis der Steuerreformtheorien

5.1. Ein Vergleich der normativen Grundlagen der Steuerreformtheorien

109

5.2. Die Beurteilung des politischen Prozesses in der Steuerreformdiskussion

110

6. Aktuelle und optimierte Steuersysteme 111

7. Zusammenfassunq

VI. OPTIMALE STEUERREFORMENTSCHEIDUNGEN BEI UNSICHERHEIT

1. Problemstellung 115

2. Das Model1 115

2.1. Das Entscheidungskriterium 116

2.2. Leviathanregeln bei Unsicherheit 121

3. Interpretation und Konsequenzen des LeviathanModel $1 \mathrm{~s}$

4. Neue Regeln für den Leviathan?

VII. ZUR POSITIVEN FUNDIERUNG STAATLICHEN VERHALTENS

IM POLITISCH-OKONOMISCHEN STEUERREFORMPROZESS

1. Die Bedeutung fiskalischer Restriktionen für den finanzpolitischen Entscheidungsprozeß

1.1. Fiskalische Restriktionen in der Demokratie

1.2. Geltende Finanzverfassungen

1.3. Weitere Restriktionen staatlicher Steuerreformentscheidungen

1.4. Die staatliche Zielfunktion

1.5. Parteienwettbewerb und wachsender politischer Handlungsspielraum

1.5.1. Das relative Wachstum von Ausqaben und Einnahmen des Staatssektors aus empirischer Sicht 
Se ite

1.5.2. Ein Leviathan-Reformmodell unter Berücksichtigung von Wählerinteressen

1.5.3. Mehrheitswahlsysteme und wachsender finanzieller Handlungsspielraum der Politiker

1.5.4. Der Parteienwettbewerb und die politisch opportune Umverteilung von Steuerlasten

1.5.5. Bürokratietheorie und wachsende Budgets

2. Die Rolle der Steuerzahler im SteuerreformprozeB 159

2.1. Das Konsumentenverhalten

2.2. Das Produzentenverhalten

2.3. Die Rolle von Interessengruppen im SteuerreformprozeB

2.4. Individuelle Verteilungsvorteile als Steuerreformziel

2.5. Die Allokations- und Verteilungswirkungen von Steuerverguinstigungen

2.5.1. Zur Definition individueller Steuerprivilegien

2.5.2. Die Allokationswirkungen

2.5.3. Kapitalisierungseffekte und Steuerreform 176

2.5.3.1. Der Marktkapitalisierungseffekt

2.5.3.2. Der institutionelle Kapitalisierungseffekt 178

2.6. Zur Dynami.k von Steuerreformen

3. Konzeptionelle Mängel des politisch-ökonomischen Steuerreformprozesses

4. Zusammenfassung

\section{VIII.STEUERVERFASSUNG ALS STEUERREFORMPROGRAMM}

1. Die Funktion einer Steuerverfassung oder die Verfassungsregeln als institutionelle SecondBest-Reqein

1.1. Einstimmigkeit und Effizienz von Verfassungsregeln

1.2. Einstimmigkeit und Gerechtigkeit von Verfassungsregein

1.3. Verfassungsregeln und individuelle Freiheit

2. Konstitutionelle Steuerregeln für die Demokratie 197

2.1. Die Wahl der Steuerbemessungsgrundlage 198

2.2. Restriktionen für Steuersätze und Steueraufkommen 
Se ite

2.3. Die Forderung nach horizontaler Gerechtigke it

2.4. Das Gleichheitsprinzip von Hayek oder konstitutionelle Regeln für Steuerstrukturen

201

2.5. Die Integration von Steuer- und Ausgabenentscheidungen

204

2.6. Das Aquivalenzprinzip

206

2.7. Die Zweckbindung öffentlicher Einnahmen

207

2.8. Der materielle Haushaltsausgleich

208

2.9. Föderalismus und die Macht der Besteuerung

209

2.10. Sunset Legislation

210

2.11. Zur Notwendigkeit weiterer Verfassunasregeln

3. Die Forderung nach konstitutionellen Grenzen für die Steuerprivilegpolitik

3.1. Eine Verfassungsregel für Steuerprivilegien 214

3.2. Vorteile einer konstitutionellen Steuerprivilegregel

IX. ZUSAMMENFASSUNG

A: Zur Interpretation der Lagrange-Multiplikatoren 229

$B$ : Definition und Messung der Mehrbelastung

$C$ : Pauschalsteuern als Kennzeichen der direkten Besteuerung

D: Zur Ableitung der "Optimierungsergebnisse" (IV.14) mit Hilfe des dualen Maximierungsansatzes

E: Die Berechnung eines Kriteriums zur schrittweisen Erhöhung des Steueraufkommens

F: Zur Ableitung von Gleichung (VII.20) 
Se ite

Abbildung 1: Systematik der optimalen Steuertheorie

Abbildung 2: Ergebnisse alternativer Gerechtigkeitskriterien

Abbildung 3: Politische okonomie von Steuerreformen

Abbildung B: Das EV-Mehrbelastungsmaß im Falle einer Steuer auf Gut $x$

\section{VERZEICHN IS DER TABELLEN}

Tabelle 1: Entwicklung der Statsausgabenquote in der Bundesrepublik Deutschland

Tabelle 2: Entwicklung der Gesamtsteuerquote in der Bundesrepublik Deutschland 
I. EINLEITUNG

I.1. Problemstellung

Der Ruf nach Verbesserungen des Steuersystems ist permanenter Bestandteil in der politischen Diskussion aller gesellschaftichen Gruppen. Aufgrund ihrer differierenden ökonomischen Positionen beurteilen sie die Güte einer Steverreform naturgemäß unterschiedlich. Während die Zensiten ihre individuelle Steuerlast zu mindern trachten und daher insbesondere neu auferlegten Steuerzahlungen regelmäßig ablehnend gegenüberstehen, verfolgen Politi ker weitgehend das fiskalische Zie ${ }^{1}{ }^{1}$. Höhere Steuereinnahmen bedeuten für sie größere finanzielle und politische Entscheidungsspielräume, die sie zur Verfolgung ihrer Ziele benötigen. Man darf also getrost erwarten, daß die Beurteilung einer Steuerreform durch die Betroffenen beinahe ausschließlich durch ihre eigenen Vor-bzw. Nachteile diktiert wird. Aus diesem Grund wird hier auf eine enge, normbezogene Definition des Begriffs Steuerreform verzichtet. Stattdessen werden unter Steuerreformen alle strukturellen Anderungen des Steuersystems und der Steuergesetzgebung subsumiert.

Im Gegensatz zur interessenorientierten Finanzpolitik war es seit jeher Aufgabe der Finanzwissenschaft ${ }^{2}$ ), ein für die gesamte Volkswirtschaft rationales Steuersystem zu konzipieren. Rationale Steuersysteme sind dadurch gekennzeichnet, daß sie eine bestmögliche Annäherung an vorgegebene wirtschaftspolitische Ziele erlauben. Die Wahl der Zielvorgabe sowie die Festlegung von Zielprioritäten durch Politiker oder Wissenschaftler implizieren grundsätzlich Werturteile, die mit zunehmender Konkretisierung

1) Nach dem fiskalischen (budgetären) Ziel dienen Steuern dazu, "Deckungsmittel für diejenigen öffentlichen Ausgaben zu beschaffen, die nicht anderweitig finanziert werden können oder sollen". Neumark (1970), S. 19

2) In diesem Zusammenhang sind vor allem die Bestrebungen der deutschen Finanzwissenschaft anzumerken: Mann (1937), S. $115 \mathrm{ff}$. , Wagner (1890), Schäffle (1895), Gerloff (1926), Ritsch1'(1925), Schmölders (1949), Neumark (1970), Hailer (1971). Alteren Datums sind die vorklassischen Steuerregeln von Smith (1776). 
umstrittener werden. Die geringsten Meinungsunterschiede verursacht die Forderung nach einem effizienten Steuersystem, das die optimale Allokation der Ressourcen einer Volkswirtschaft am wenigsten beeinträchtigt. Dagegen wird die Diskussion um ein geeignetes Gerechtigkeitskriterium sehr heftig geführt ${ }^{1}$ ).

Obwohl rationale Steuersysteme durch eine Vielzahl weiterer Normen gekennzeichnet sein können ${ }^{2)}$, wird seit Anfang der siebziger Jahre vor allem im angelsächsischen Sprachraum die Analyse optimaler Steuern uberwiegend unter Effizienz- und Verteilungsaspekten vorgenommen. Wegen ihrer überragenden ökonomischen Bedeutung werden Allokations- und Distributionsziele auch im Mittelpunkt dieser Untersuchung stehen.

Im Gegensatz zu der traditionellen Vorgehensweise will die Theorie der optimalen Besteuerung (OT) rationale Steuersysteme in allgemeinen Gleichgewichtsmodellen entwerfen, die alle als relevant erachteten ökonomischen Beziehungen in Form von Gleichungen beinhalten. Obwohl der hohe Abstraktionsgrad häufig kritisiert wird, hat er den großen Vorteil, daß der Wissenschaftler alle für seinen Untersuchungszweck irrelevanten systembeziehungen vernachlässiạen kann.

Eine wesentlich gravierendere Kritik liegt auf der konzeptionellen Seite. Da die OT im Rahmen der normativen Theorie den Anspruch erhebt, Argumentationshilfen und Richtlinien für politische Entscheidungen liefern zu wollen, stellt sich die Frage, warum die Ergebnisse dieser klar strukturierten Analyse in der finanzpolitischen Praxis eine so geringe Rolle spielen. Obwohl in der Literatur bezüglich der Zielsetzung der OT unterschiedliche Auffassungen vertreten werden, darf man unterstellen, daß mit ihrer Hilfe letztendlich die Steuergesetzgebung über exakte

1) Den Kern des Problems skizziert Grossmann wie folgt:"daß Streit um die Gerechtigkeit im öffentlichen Haushalt ein Streit um materielle Interessen ist" (und) "daß jeder als gerechte Steuer die Steuer betrachtet, die von anderen bezahlt wird". Grossmann (1948), S. 35. Vgl. zu einer Diskussion neuerer Kriterien steuerlicher Gerechtigkeit Kap. IV dieser Arbeit.

2) Vgl. z.B. Neumark (1970), Haller (1971) 
durchstrukturierte Ziel-Mittel-Beziehungen beeinfluBt werden soll; ohne dieses Ansinnen wäre ihr ohnehin schon angezweifelter gesellschaftspolitischer Wert noch weniger deutlich.

Die Diskrepanz zwischen dem Anspruch der OT als Argumentationsbasis beabsichtigter Steueränderungen und ihrer gegenwärtig desillusionierenden Bedeutungslosigkeit in der steuerpolitischen Diskussion kann weitgehend durch konzeptionelle Mängel erklärt werden; diese bestehen vor allem in der Vernachlässigung der Informationsproblematik und politisch-ökonomischer Zusammenhänge. Damit stelit sich aier auch die Frage, welche Rolle die OT in der praktischen Finanzpolitik tatsächlich spielen kann. Denn die Ergebnisse einer Theorie, die von aktuellen Entscheidungsmechanismen abstrahiert, lassen sich im demokratischen WillensbildungsprozeB leicht miBbrauchen ${ }^{1)}$. Diese Gefahr besteht für die OT; sie operiert in einem politischen Vakuum, unterstellt aktionsarme Wirtschaftssubjekte und klammert deshalb das Problem der aktuellen Durchsetzbarkeit ihrer zum Teil wertvollen Ergebnisse - nicht zuletzt auch wegen des analytisch höchst anspruchsvollen $\mathrm{Ni}$ veaus ${ }^{2)}$ - aus.

Die vorliegende Arbeit greift einige Defizite der OT auf und versucht, durch Erweiterungen der Modellprämissen und durch die Modifikation einiger Verhaltenshypothesen herauszuarbeiten, welche Faktoren bei der Durchsetzung gesellschaftlich rationaler Steuerreformen von ausschlaggebender Bedeutung sind. Gleichwohl bleibt die OT das Referenzsystem einer rationalen Steuerpolitik; nur ein Vergleich zwischen demaktuellen und einem idealen Systemerlaubt ein Abwägen der Wirkungen aktueller Steueränderungen auf Effizienzund Verteilungsziele. Darüber hinaus kann die OT auch zur Analyse von Anderungen des wirtschaftlichen Datenkranzes dienen, die aus politischen oder konstitutionellen Reformabsichten resultieren ${ }^{3}$.

1) Vgl. dazu Frey (1976)

2) Vgl. Z.B. Schmidt (1980), S. 156

3) Vgl. Z.B. die Analysetechnik bei Brennan/Buchanan (1980) 
Deshalb wird zunächst die Vorgehensweise der OT vorgestellt.

Vorschläge zur operationalen Umsetzung normativer Ergebnisse in politische Handlungsanweisungen erfordern jedoch einen positiven politisch-ökonomischen Modellansatz. Anders als in der OT wird gefragt: Welche institutionellen Hemmnisse stehen rationalen Steuerreformen im Wege? Auf diese Weise werden Gründe für permanent unbefriedigende Steuerreformen offengelegt und Konzepte abgeleitet, die vom steuerpolitischen EntscheidungsprozeB als Grundlage zielorientierter Reformansätze ausgehen und daher auch die Chance für die Verwirklichung einiger der zuvor abgeleiteten qualitativen Ergebnisse beinhalten.

Unter dem Gesichtspunkt eigennütziger Steuerpolitiker setzen Brennan/Buchanan ${ }^{1)}$ der OT eine konstitutionelle Steuertheorie entgegen. Ihre konstitutionellen Steuerregeln sollen als Kontrolle staticher Steuermacht fungieren. Die Ergebnisse der konstitutionellen Steuertheorie stehen den OT-Regeln diametral gegenüber. Es stellt sich somit die Frage, welches der beiden Theoriegebäude die geeignetere Basis für finanzpolitische Reformempfehlungen liefert. Die Antwort darauf kann nur mit Hilfe eines positiven Modellansatzes gefunden werden. Es gilt daher, den Ablauf demokratischer Steuerreformprozesse zu analysieren. Dies impliziert eine exakte Spezifikation sowohl statiicher als auch individueller Steuerreformaktivitäten, um die Relevanz der konträren Theoriegebäude besser einschätzen zu lernen. Die positive Fundierung deckt wesentliche Schwächen des finanzpolitischen Willensbildungsprozesses in der Demokratie auf und führt zu Reformvorschlägen, die eine zieladäquate Steuerpolitik gewährieisten sollen.

\section{I.2. Gang der Untersuchung}

Die Kapitel II bzw. IV enthaltendie Analyse der Effizienz-bzw.Verteilungsaspekte der OT. Die Untersuchung beginnt mit der Darstel-

1) Brennan/Buchanan (1980) 
lung des OT-Konzeptes. Danach werden einige typische Strukturen der OT anhand eines Modells erläutert, wobei besonders die Verhaltensmuster der Reformbeteiligten (Konsumenten, Produzenten und Staat) interessieren. Neben einigen bekannten Standardergebnissen der OT können mit Hilfe der Ausgabenfunktion - ein in der deutschsprachigen finanzwissenschaftlichen Literatur bisher noch wenig verwendetes mikroökonomisches Instrumentarium - einige wichtige Erkenntnisse für die optimale schrittweise Reform eines Steuersystems entwickelt werden. Aufgabe des III. Kapitels ist es, die Informationsproblematik der OT-Modelle hervorzuheben. Dabei werden die wesentlichen Vor- und Nachteile der OT erörtert, und es wird gezeigt, daB sie allein keine geeignete Basis zur Ableitung finanzpolitischer Empfehlungen sein kann. Kapitel IV befaßt sich mit der Diskussion um den Konflikt zwischen Effizienzund Verteilungsaspekten in der OT. Die hieraus resultierenden Reformempfehlungen unterscheiden sich erwartungsgemä $B$ von jenen eines Ein-Konsumenten-Modells. Ferner wird deutlich, daß die Ergebnisse eines optimalen Steuersystems ganz entscheidend von den zugrundeliegenden Gerechtigkeitskriterien abhängen. In Kapitel V wird die konstitutionelle Theorie der Besteuerung von Brennan/Buchanan als Gegenposition zur OT auf der Basis eines Leviathan-Modells entwickelt. Von der Arbeitshypothese eines aufkommenmaximierenden Steuerstaates ausgehend werden einige fiskalische Verfassungsregeln abgeleitet sowie deren Vor- und Nachteile dargelegt. Kapitel V schließt mit einer empirischen Betrachtung aktueller Steuersysteme und einer kritischen Würdigung der Leviathanhypothese. Da die Leviathanregeln mit den aktuellen Steuerstrukturen nicht übereinstimmen, wird in Kap. VI der Versuch unternommen, diese Diskrepanz durch die Einbeziehung unsicherer Erwartungen über die Konsumentennachfrage zu erklären. Es wird ein Leviathanmodell bei Unsicherheit konzipiert, das zeigt, daB einige der Abweichungen zwischen aktuellen und optimierten Steuersystemen auf die Problematik von Informationsdefiziten im finanzpolitischen Planungsprozeß zurückzuführen sind. Dennoch stellt dieses Entscheidungsmodell bei Unsicherheit nur eine erste Annäherung an den aktuellen politischen Willensbildungsprozeß dar, weil es eine zu simple Zielfunktion des Fiskus unterstellt und 
keinerlei Wahlprozesse berücksichtigt. Im anschlieBenden Kapitel wird der Leviathanhypothese eine positive Analyse des staatlichen Verhaltens im politisch-ökonomischen SteuerreformprozeB gegenübergestellt, die die wesentlichen Charakteristika politischer Entscheidungsprozesse in der Demokratie aufzeigt und daher einen höheren Erklärungsgehalt als das Leviathanmodell für sich in Anspruch nehmen darf.

Abgesehen von einer positiven Fundierung staatlichen Verhaltens mangelt es der OT und der konstitutionellen Theorie der Besteuerung vor allem auch an einem Erklärungsmodell des Steuerzahlerverhaltens im Reformprozeß. In Kap. VII wird daher der Versuch unternommen, diese Lücke im Rahmen des politisch-ökonomischen Ansatzes zu schließen. Dabei zeigt sich, daß der demokratische Steuerreformprozeß sowohl durch eine allmähliche Erhöhung des Steueraufkommens als auch durch viele unsystematische und ineffiziente Entscheidungen gekennzeichnet ist.

Auf der Basis der vorangegangenen Untersuchungen wird im letzten Kapitel die Notwendigkeit von fiskalischen Verfassungsregeln herausgestellt, die insbesondere der Unsystematik des demokratischen Steuergesetzgebungsprozesses entgegenwirken sollen. Erst in zweiter Linie dienen sie der Begrenzung des Steueraufkommens. Nach der Analyse der Logik und Funktion dieser Regeln werden einige der bekanntesten fiskalischen Beschränkungen und ihre Wirkung auf den demokratischen ReformprozeB untersucht. Zum AbschluB der Arbeit wird eine Verfassungsregel zur systematisierung von Steuerprivilegien entwickelt. Mit ihrer Hilfe soll die Vergabe von Steuerprivilegien nach politischer opportunität verhindert und somit ein charakteristischer Mangel der demokratischen Steuerpolitik behoben werden. 


\section{OPTIMALE STEUERREFORMEN UNTER EFFIZIENZASPEKTEN}

In der wissenschaftlichen Steuerreformdiskussion spielt die Theorie der optimal taxation (OT) seit über einem Jahrzehnt, insbesondere in der angelsächsischen Literatur, eine bedeutende Rol1e. Sie stellt den Versuch einer Ergänzung und Weiterentwicklung der traditionellen normativen Steuerlehre unter Verwendung mathematischer Methoden dar.

Trotz bemerkenswerter theoretischer Fortschritte ist es dieser speziellen Ausprägung der normativen Steuerlehre nicht gelungen, einen erkennbaren EinfluB auf die praktische Steuerpolitik auszu-

üben ${ }^{1)}$. Dabei kann der theoretische Erkenntniswert der OT kaum bestritten werden. Erst durch diese formale Analyse werden viele Einsichten in die ökonomischen Wirkungszusammenhänge komplexer Steuersysteme offenbar.

Es stellt sich daher zwangsläufig die Frage, welche Gründe für die geringe Resonanz auBerhalb der theoretischen Finanzwissenschaft verantwortlich sind.

In diesem Zusammenhang lassen sich mehrere Punkte kritisieren:

(1) der mathematische Formalismus ${ }^{2}$ )

(2) die Einfachheit ${ }^{3}$ ) und Irrelevanz der Annahmen

(3) der auBerordentlich hohe Informationsbedarf 4 )

(4) die Vernachlässigung von Steuerinstitutionen ${ }^{5}$ )

1) Schmidt (1980), S. 156 "wegen der 'Sprachlosigkeit' im Hinblick auf anfällige Steuerreformen hat dieser neue Ansatz der Steuerlehre nicht selten reservierte Aufnahme und auch deutliche Kritik gefunden." Oder Folkers (1983b), S. 189

2) Schmidt (1980), S. 156

3) Vgl. zur Kritik an dieser Kritik Seidl (1983), S. $210 \mathrm{f}$.

4) "The central element in the theory is information," Mirrlees (1977), S.2

5) Vgl. Brennan/Buchanan (1980), S. 34 
(5) die exogene Betrachtungsweise des politischen Entscheidungsprozesses $^{1 \text { ) }}$

Im folgenden soll geprüt werden, ob die eben skizzierten Kritikpunkte prinzipiell berechtigt sind. Zu diesem Zweck wird zunächst die Konzeption der OT dargestellt und konkretisiert, welche Informationen zur Quantifizierung optimaler Steuerregein erforderlich sind. Dabei soll mit Hilfe eines OT-Modells demonstriert werden, daB Restriktionen der steuerlichen Rahmenbedingungen, d.h. Begrenzungen der Steuerbemessungsgrundlage und der Steuersätze, zu erheblichen Anderungen optimaler Steuerstrukturen führen können. Diese Oberlegungen verdeutlichen nicht nur die Abhängigkeit der OT-Empfehlungen von den speziellen Modellannahmen; sie führen darüber hinaus zu der Erkenntnis, daß in einer normativen Steuerlehre auch der politisch-ökonomische ProzeB und das daraus resultierende Spektrum potentieller Steuerinstitutionen berücksichtigt werden müssen, um steuerpolitisch relevante Empfehlungen zu erhalten.

Vorab sei aber schon darauf hingewiesen, daB das Abstraktionsniveau einer Theorie und die Verwendung mathematischer Methoden a priori keine Kriterien für oder gegen die Qualität der daraus resultierenden Ergeonisse sein können. In jeder ökonomischen Analyse muß formal oder verbal von bestimmten Gegebenheiten der Realität abstrahiert werden, um die wesentlichen Aspekte des betrachteten Problems herauszufiltern ${ }^{2}$ ). Mit zunehmender Annäherung an die Realität werden die verbalen bzw. formalen Modelle komplexer, weil die Zahl der Einflußfaktoren steigt. Die Forderungen nach Realitätsnähe und Modelleinfachheit stehen sich daher meist diametral gegenüber. Der hohe mathematische Aufwand in den OT-Modellen wird damit begründet, "daB die Mathematik ein nützliches Hilfsmittel bei der Analyse ökonomischer Probleme sein kann und unvoreingenommen dort verwendet werden sollte, wo der Ein-

1) Folkers (1983b), S. $189 \mathrm{ff}$.

2) Vgl. zur Diskussion der Grundsatzprobleme mathematischer Modelle z.B. Brunner (1984), S. $308 \mathrm{ff}$. 
satz eine schnellere und leichtere Durchdringung komplexer ökonomischer Probleme erlaubt als eine rein verbale oder graphische Argumentation"1). Soweit mathematische Methoden in dieser Arbeit verwandt werden, finden sie ihre Rechtfertigung in dieser Argumentation.

\section{Das Konzept der Theorie der optimalen Besteuerung}

Bevor politische oder institutionelle Aspekte rationaler Steuerreformen analysiert werden können, müssen zunächst die ökonomischen Wirkungszusammenhänge von Steueränderungen bekannt sein. Für die Aufdeckung dieser Zusammenhänge ist die Theorie der optimal taxation in hervorragender Weise geeignet. Die Stichworte optimal taxation (OT). Theorie der optimalen Besteuerung oder Neue Finanztheorie werden synonym verwandt und stehen für die streng formalisierte Behandlung der herkömmlichen finanzwissenschaftlichen Frage, wie ein gutes (optimales) Steuersystem ausgestaltet sein sollte. Die OT-Modelle basieren auf den Annahmen der allgemeinen Gleichgewichts-und Wohlfahrtstheorie. Die mikroökonomisch fundierte Theorie der Besteuerung unterstellt Konsumentensouveränität und vollkommene Wettbewerbsmärkte, die dann ein Pareto-Optimum gewährleisten, wenn die Nutzen und Produktionsfunktionen der Konsumenten und Produzenten geeignete Eigenschaften besitzen ${ }^{2}$. Unter diesen idealen Voraussetzungen würde die Verfolgung des Allokationszieles keine staatlichen Eingriffe in den Wirtschaftsprozeß rechtfertigen. Offensichtlich existieren jedoch viele Arten des Marktversagens, die eine effiziente Allokation der Ressourcen verhindern und ein statiches Güterangebot erforderlich machen ${ }^{3)}$. Will der stat beispielsweise öffentliche Güter bereitstellen, muß er zuvor durch den Einsatz seiner steuerlichen Instrumente in die Lage versetzt werden, Ressourcen vom privaten in den öffentlichen Sektor zu transferieren. Die Erzielung von Einnahmen ist unter diesem Aspekt

1) Rose/Wiegard (1983), S. 128

2) Vgl. dazu Z.B. Varian (1981), Kan. 3

3) Vgl. Bernholz (1972), Kap. 5 
kein eigenständiges Ziel rationaler Finanzpolitik; sie isteine Vorbedingung für die Erfüllung der Allokationsaufgabe ${ }^{1}$ ). Damit ist allerdings noch nichts über die Höhe eines optimalen Steueraufkommens bzw. Ausgabenniveaus und über eine optimale Steuerstruktur gesagt.

Die wesentlichen Zusammenhänge des Konzepts der OT werden in Abb. 1 graphisch verdeutlicht. Außerhalb des symbolisch angedeuteten Modellrahmens sind jene Informationen aufgereiht, die einem allwissenden Diktator oder dem Planungsbüro modellexogen zufließen müssen, damit sie ihre Optimierungsaufgabe erfüllen können (Pfeil 1). Implizit wird daher unterstellt, daB die mit diesen Vorgaben verbundenen Informations- und Entscheidungsprobleme bereits gelöst sind.

Zu den wichtigsten Informationen gehort die Kenntnis des optmalen Niveaus öffentlicher Güter und Dienstleistungen ${ }^{2}$ ). Bei gegebenen Preisen ist damit auch das optimale Ausgabenniveau bzw. Steueraufkommen determiniert.

Weiterhin müssen die Ziele bzw. Normen der Besteuerung bekannt sein. Es muß beispielsweise entschieden werden, ob das rationale Steuersystem ausschlieBlich nach Effizienz-oder aber auch nach Gerechtigkeits - und Verteilungskriterien konzipiert werden soll. Das einführende OT-Modell beschäftigt sich zunächst nur mit dem Effizienzaspekt, um später die Wirkungen der Gerechtigkeits- und Verteilungsziele auf die optimale Steuerstruktur leichter isolieren zu können. Die Vorteilhaftigkeit dieser Trennung wird besonders deutlich, wenn man später die Verteilungsaspekte in Form einer sozialen Wohlfahrtsfunktion in die Analyse einbezieht.

Ein effizientes Steuersystem ist dadurch gekennzeichnet, daß der

1) Vgl. Albers (1977), S. 130

2) Nur in wenigen Modellen wird die optimale Menge öffentlicher Güter modellendogen bestimmt. Vgl. Stiglitz/Dasgupta (1971). Die Modelle erlauben allerdings keine Quantifizierung optimaler Güterbündel. 
Staat den Nutzen eines repräsentativen Konsumenten unter Beachtung der gegebenen Restriktionen maximiert. Neben den Konsumentenpräferenzen und der Höhe des optimalen Steueraufkommens muB er vor allem berücksichtigen, welches steuerliche Instrumentarium überhaupt zur Zielrealisierung zur Verfügung steht. Die Einengung des zulässigen Steuerinstrumentariums oder der steuerbemessungsgrundlage hat erheblichen Einfluß auf die Struktur der optimalen Steuersysteme und verweist die OT in den Bereich der Theorie des Zweitbesten. Variationen der steuerpolitischen Rahmenbedingungen selbst sind jedoch das Ergebnis eines dynamischen politischen Prozesses, der nur auBerhalb des Modells erklärt werden kann, andererseits aber durch die Steuerzahler beeinfluBt wird. Auch aus diesem Blickwinkel heraus erscheint es sinnvoll und notwendig, Rückwirkungen steuerpolitischer Entscheidungen auf den politischen Entscheidungsprozeß näher zu untersuchen.

Wenn der wohlwollende Planer alle notwendigen Informationen besitzt, kann er sein spezifisches Optimierungsproblem (möglicherweise) lösen 1) (Pfeil 2). Steuerreformen werden durchgeführt, wenn die bisherige Steuergesetzgebung mit der optimalen Steuerstruktur nicht oder nicht mehr übereinstimmt. Die Reformen werden sich auf die Struktur der relativen Preise niederschlagen (Pfeil 3). Die Konsumenten und Produzenten passen sich nun den neuen Marktpreisen an (Pfeil 4). Das neue Systemgleichgewicht ist dadurch gekennzeichnet, daB weitere Steuerreformen unter den gegebenen Bedingungen zu keiner Wohlfahrtserhöhung führen können.

In Abb. 1 fällt zunächst der erhebliche exogene Informationsbedarf des "wohlwollenden Planers" auf. Besondere Aufmerksamkeit verdienen auch die (zu) einfachen ökonomischen Reaktionsmuster der Steuerzahler und das uneigennützige Verhalten der Regierung. Produzenten und Konsumenten sind völlig apolitisch und haben keinen Einfluß auf die steuerpolitischen Entscheidungen des Staates. Genauer gesagt, in den Modellen der OT fallen keine politischen

1) Möglicherweise deshalb, weil nicht alle Optimierungsaufgaben Lösungen bzw. nur eine Lösung haben. 
Abb. 1: Systematik der optimalen Steuertheorie

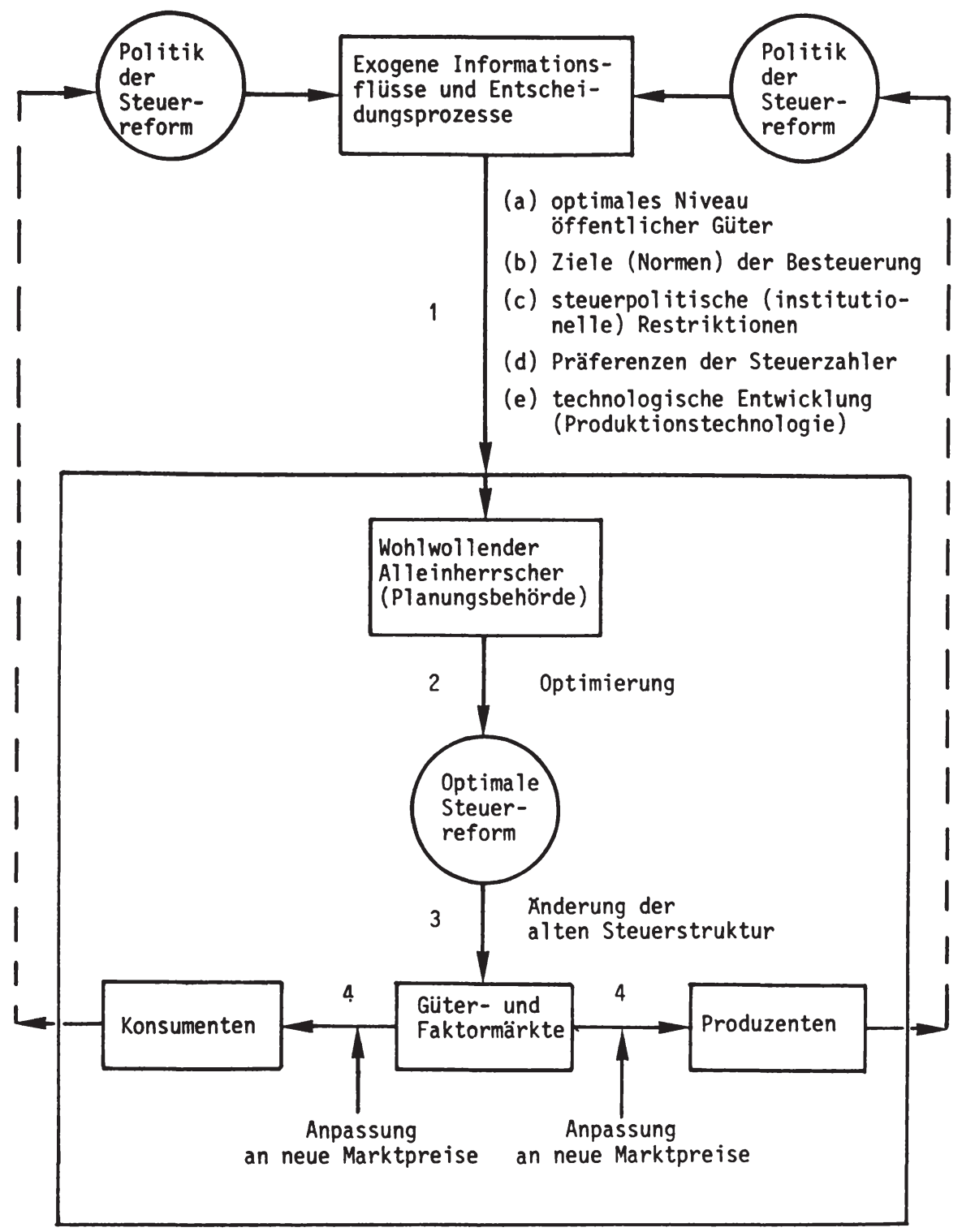


Entscheidungen, sondern ausschließlich ökonomische. Der Staat hat nur das Interesse der Steuerzahler im Auge, ohne machtpolitische Ambitionen zu entwickeln. Diese institutionelle Interpretation des OT-Konzepts impliziert eine ideale Funktionsweise des Parteienwettbewerbs, in dem letztlich nur die Wählerpräferenzen zum Ausdruck kommen. Derartige Vorstellungen von politischen Entscheidungsprozessen werden der Realität kaum gerecht.

Die normative Steuertheorie muß daher um informationstechnische, institutionelle und politische Aspekte ergänzt werden. Dadurch ergeben sich z.T. erhebliche Modifikationen gegenüber den OT-Empfehlungen. Die Grundlage der weiteren Diskussion rationaler Steuerreformen ist ein einfaches OT-Modell, das in vielerlei Hinsicht modifiziert werden kann und daher mehreren Untersuchungszwecken dient. Am Beispiel dieses Modells wird konkretisiert, welchen EinfluB alternative Steuerinstrumente, institutionelle Verhaltensweisen und Informationsmängel auf die optimale Steuerplanung haben. Die Ergebnisse führen zu dem Schluß, daß die exogenen Informations- und Entscheidungsprozesse ( $A b b$. 1) in einer umfassenden Steuerreformanalyse nicht vernachlässigt werden dürfen, da sie selbst durch die modellendogenen optimierungsprozesse tangiert werden können.

\section{II.2. Die Modellstruktur}

Die Allokationsaspekte der OT können mit Hilfe des Konzeptes der Ausgabenfunktion ${ }^{1)}$ untersucht werden. Die Ausgabenfunktion gibt die Mindestausgaben an, die ein Konsument bei gegebenen Marktpreisen aufbringen muß, um ein bestimmtes Nutzenniveau zu realisieren. Dieser "duale"2) mikroökonomische Ansatz wurde vor allem

1) Eine umfassende Anwendung der Ausgabenfunktion in der or geht auf Dixit (1975) zurück. Weitere wichtige Beiträge stammen von Dixit/Munk (1977), Munk (1978) und Munk (1980).

2) Diamond/Mc Fadden (1974) führten dieses Konzept erstmals in die theoretische Finanzwissenschaft ein. Die folgenden Ausführungen zur Dualität beruhen im wesentlichen auf Varian (1981). Grundlegend sind die Arbeiten von Hotelling (1935), Roy (1947), Samuelson (1947), KcKenzie (1957). Wichtig sind auch die neueren Ergebnisse von Darough/Southey (1977), Deaton (1979), Blackorby/Diewert (1979), Weymark (1980), Diewert (1982) 
in der deutschsprachigen Literatur bisher weitgehend vernachlässigt, obwohl sein Pendant in der Produktionstheorie schon lange bekannt ist ${ }^{1}$. Dualität ${ }^{2}$ zwischen der Ausgabenfunktion und der indirekten Nutzenfunktion ${ }^{3}$ ) bedeutet, daß beide Funktionen das Präferenzsystem eines Konsumenten (unter bestimmten Bedingungen) eindeutig beschreiben. Daher ist mit der Formulierung einer Ausgabenfunktion auch eine direkte (und indirekte) Nutzenfunktion ${ }^{4}$ ) beschrieben, die den üblichen mathematischen Anforderungen genügt. Die Wahl eines optimalen Faktor- und Konsumgüterbündels kann somit durch zwei alternative optimierungsansätze demonstriert werden.

Obwohl die Regierung mit der Besteuerung normalerweise mehrere wirtschaftspolitische Ziele verfolgen wird, beschränkt sich die folgende Analyse auf das Allokationsziel der Besteuerung. Dies ist auch deshalb notwendig, weil die Ausgabenfunktion kein geeignetes Instrument zur Untersuchung von Distributionsfragen ist ${ }^{5)}$; dafuir ist sie in der Effizienzanalyse um so wirkungsvoller. Um einsichtige Steuerregeln herzuleiten, sollte das Modell nicht nur eine formal durchschaubare Struktur erhalten, sondern auch die wesentlichen Sektoren einer Volkswirtschaft umfassen und diese klar strukturieren. Zu diesem Zweck teilt sich das in dieser Arbeit konzipierte Modell in drei Aktivitätszentren: Konsum-, Produktions- und Statssektor.

1) Eine Ausnahme findet sich bei Wiegard (1978), S. $100 \mathrm{ff}$.

2) Streng davon zu unterscheiden ist der mathematische Dualitätsbegriff. Der Begriff wird hier trotzdem verwendet, weil er sich in der Literatur etabliert hat.

3) Die indirekte Nutzenfunktion verwenden beispielsweise: Dixit (1970), Diamond/Mirrlees (1971a und b), Stiglitz/Dasgupta (1971), Green (1975), Sandmo (1976) und Atkinson/Stiglitz (1980). Ramsey (1927) verwandte die direkte Nutzenfunktion. Die einzelnen Begriffe werden im folgenden noch näher erläutert.

4) In den bekannten Modellen der OT wird üblicherweise die indirekte Nutzenfunktion zur Ableitung optimaler Steuersysteme verwendet. Vgl. beispielsweise Dixit (1970)

5) Das Problem liegt in der ungewichteten Aggregation der individuellen Wohlfahrtsverluste, wodurch Verteilungsaspekte im Gegensatz zur sozialen Wohlfahrtsfunktion ausgeklammert werden. Vgl. Z.B. Tresch (1981), S. $85 \mathrm{ff}$. 


\section{II.2.1. Der Konsumsektor}

Um den Effizienzaspekt der OT-Analyse vollkommen zu extrahieren und um alle Aggregationsprobleme zu vermeiden, sei von der Fiktion eines repräsentativen Wirtschaftssubjektes ausgegangen ${ }^{1)}$. Dieser Konsument zeichnet sich vor allem durch passives Reagieren auf alle ökonomischen und politischen Anderungen in einer dem Planer vorher bekannten Art und Weise aus. Er hat keinen Einfluß auf steuerpolitische Entscheidungen und tritt als Wähler nicht in Erscheinung. In Kapitel VII wird diese Hypothese aufgehoben und ein realistischeres Bild der Konsumentenreaktionen auf steuerpolitische Entscheidungen gezeichnet. Zunächst beschränken sich seine Aktivitäten auf das folgende Optimierungsproblem.

\section{II.2.1.1. Das primale Optimierungsproblem}

Der Konsument maximiere seine Nutzenfunktion

$$
\text { (II.1) } u=u\left(x_{1}, \ldots, x_{n}\right)=u(x)
$$

unter der Nebenbedingung einer gegebenen Budgetrestriktion ${ }^{2}$ )

$$
\text { (II.2) } \quad \sum_{i} q_{i} x_{i}=m \quad i=1, \ldots, n
$$

wobei u das Nutzenniveau, $x=\left(x_{1}, \ldots, x_{n}\right)$ den Güter- und Faktormengenvektor, $q=\left(q_{1}, \ldots, q_{n}\right)$ den Konsumentenpreisvektor und $m$ einen fixen Einkommensbestandteil (z.B. aus Gewinneinkommen, staatlichen Transfers oder fixem Faktorangebot) bezeichnen.

1) Aquivalent wäre die Vorstellung von Individuen, die in allen ökonomischen Belangen (Nutzenfunktion, Einkommen U.s.w.) identisch sind.

2) Der Güter- und Faktormengenindex $i$ läuft hier und im folgenden von 1 bis $n$, so daB auch das Summenzeichen als $\sum^{n}$ zu lesen ist, sofern keine Anderungen angegeben werden. 
Guiter- und Faktormengen unterscheiden sich in der formalen Handhabung dadurch, daß die Faktoren mit negativem Vorzeichen in die Modellgleichungen eingehen. Als Ergebnis des Maximierungsansatzes erhält man die Konsumgüternachfrage-bzw. Faktorangebotsfunktionen des Individuums

$$
\text { (II.3) } \quad x_{i}=x_{i}(q, m) \text {. }
$$

Aus (II.1) und (II.3) resultiert die indirekte Nutzenfunktion, die die Abhängigkeit des Nutzenniveaus von den Konsumentenpreisen und $m$ widerspiegelt:

$$
\text { (I I.4) } u[x(q, m)] \equiv v(q, m)
$$

Die Inverse der indirekten Nutzenfunktion bei gegebenem Preisvektor q wird als Ausgabenfunktion bezeichnet:

$$
\text { (II.5) e }(q, u) \text {. }
$$

Die Funktion $e(q, u)$ gibt die Minimalausgaben an, die ein Konsument beim Preisvektor q aufbringen muß, um ein bestimmtes Nutzenniveau u zu realisieren ${ }^{1)}$. Bezeichnet man das maximale Nutzenniveau aus (II.1) und (II.2) mit $u^{\star}$ und gilt $u^{\star}=\bar{u}$ bei identischem Preisvektor $q$ in (II.3) und (II.5), dann stimmen die Minimalausgaben zur Erzielung von $\bar{u}$ gerade mit m überein.

\section{II.2.1.2. Das duale Optimierungsproblem}

Gleichung (II.5) kann auch durch folgende Minimierungsaufgabe, die in der Literatur üblicherweise als Dualitätsansatz bezeichnet wird, abgeleitet werden:

$$
\text { (II.6) min.: } \underset{i}{. \sum_{i}} \mathrm{x}_{i}
$$

1) Vgl. zur Interpretation Z.B. Varian (1981), S. $92 \mathrm{ff}$. 
(II.7) u.d.N.: $u(x)=\bar{u}$.

Aus der korrespondierenden Lagrange-Funktion

(II.8) $L=\sum_{i} q_{i} x_{i}+\xi[\bar{u}-u(x)]$

resultieren die Bedingungen erster Ordnung

(II.9) $\frac{\partial L}{\partial x_{i}}=q_{i}-\xi \frac{\partial u(x)}{\partial x_{i}}=0$

und

(II.10) $\frac{\partial L}{\partial \xi}=\bar{u}-u(x)=0$.

Löst man das Gleichungssystem (II.9), (II.10) nach den Variablen $x_{i}$ auf, so erhält man die einkommenskompensierten Angebots- und Nachfragefunktionen ${ }^{1}$ ).

(II.11) $x_{i}^{c}=x_{i}(q, \bar{u})$.

Setzt man (II.11) in die Zielfunktion (II.6) der Minimierungsaufgabe ein, resultiert die Ausgabenfunktion

(II.12) $e(q, \bar{u})=\sum_{i} q_{i} x_{i}(q, \bar{u})=m$,

deren numerischer Wert dem Pauschaleinkommen m in (II.2) entspricht, sofern $\bar{u}=u^{*}$ und $q$ dem Preisvektor des primalen Optimierungsproblems gleicht. Die ersten partiellen Ableitungen der Ausgabenfunktion nach ihren Argumenten $q_{j}$ entsprechen genau den

1) Die Begriffe einkommenskompensierte, konditionale bzw. Hicks'sche Angebots- und Nachfragefunktionen werden in der Literatur synonym verwandt. 
kompensierten Angebots- bzw. Nachfragefunktionen ${ }^{1)}$ :

$$
\frac{\partial e(q, \bar{u})}{\partial q_{i}}=x_{i}(q, \bar{u})=x_{i}^{c} .
$$

Die zweiten partiellen Ableitungen der Ausgabenfunktion (oder die ersten der $x_{j}^{c}$ ) ergeben:

(I I. 14) $\quad \frac{\partial^{2} e(q, \bar{u})}{\partial q_{j} \partial q_{k}}=\frac{\partial x_{i}(q, \bar{u})}{\partial q_{k}}=\left.\frac{\partial x_{i}}{\partial q_{k}}\right|_{\bar{u}}=s_{i k}$

Sie sind mit den Substitutionseffekten der slutsky-Gleichung ${ }^{2}$ ) identisch:

$$
\frac{\partial x_{i}}{\partial q_{k}}=\left.\underbrace{\left.\frac{\partial x_{i}}{\partial q_{k}}\right|_{\bar{u}}}_{\begin{array}{l}
\text { Substitutions- } \\
\text { effekt }
\end{array}} \underbrace{-x_{k} \frac{\partial x_{i}}{\partial m}}_{\begin{array}{l}
\text { Einkommens } \\
\text { effekt }
\end{array}}\right|_{q}
$$

Bei gegebenem Preisvektor $q$ und $\bar{u}=u^{\star}$ sind die Güter-bzw. Faktormengen beider Optimierungsaufgaben im Haushaltsgleichge wicht identisch, so daB sich die normalen und kompensierten Angebots-bzw. Nachfragefunktionen schneiden. Die kompensierte Nachfrage-(Angebots-) Funktion gibt an, welche Güter-(Faktor-)Mengen der Konsument bei alternativen Preisen nachzufragen (anzubieten) bereit ist, wenn er gleichzeitig durch Pauschalsteuertransfers so kompensiert wird, daB er auf demselben Nutzenniveau verbleibt.

Aus (II.6) und (II.7) läßt sich zeigen, daß die Hicks'schen Nachfragefunktionen homogen vom Grade null in den Konsumentenpreisen sind, indem man alle Preise mit einer Konstanten multi-

1) Vgl. zu den Eigenschaften der Ausgabenfunktion z.B. Varian (1981), S. 94 ff. 2) Siehe Z.B. Henderson/Quandt (1983), S. 29 
pliziert und die Minimierungsaufgabe erneut durchführt. Daher folgt aus dem Euler-Theorem für homogene Funktionen:

( I I. 16)

$$
\sum_{k=1}^{n} q_{k} \frac{\partial x_{i}^{c}}{\partial q_{k}}=0, \quad \text { für } i=1, \ldots, n \text {. }
$$

Zusammenfassend können die bisher abgeleiteten Zusammenhänge folgendermaßen veranschaulicht werden ${ }^{1)}$ :

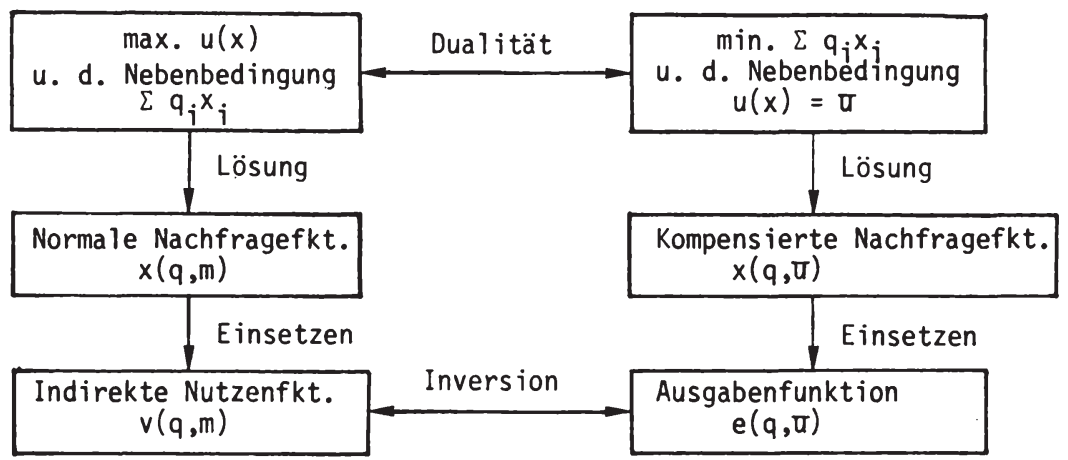

Die wesentlichen Eigenschaften der Ausgabenfunktion können wie folgt zusammengefaBt werden ${ }^{2}$ ):
(a) e $(a, u)$ ist nicht-abnehmend in $q$,
(b) $e(q, u)$ ist homogen vom Grade eins in $q$,
(c) $e(a, u)$ ist konkav in $q$ und
(d) $e(a, u)$ ist stetig in $q$ für $q>0$.

\section{II.2.2. Der Produktionssektor}

Da in dieser Arbeit vorrangig Besteuerungsprobleme auf der Konsumentenseite analysiert werden sollen, wird von produktionstechni-

1) Die Eraphik folgt der Darstellung bei Deaton/Muellbauer (1980), S. 38

2) Varian (1981), S. 94 
schen Schwierigkeiten abstrahiert ${ }^{1)}$, so daß von effizienter Produktion im privaten Bereich ausgegangen werden soll. Diese strenge Voraussetzung kann in der Realität nicht aufrecht erhalten werden. Das impliziert, daß der Fiskus zusätzliche Informationen benötigt, um seine Optimierungsaufgabe zu lösen. Die Vereinfachung erscheint hier trotzdem gerechtfertigt, weil sie die spätere Argumentation nicht tangiert.

Die Produktionsmöglichkeiten der privaten Unternehmer lassen sich demnach durch eine aggregierte Produktionsfunktion beschreiben:

$$
(\text { II.17) } \quad F=F(y) \text {, }
$$

wobe $i$

$$
\left(\text { II.18) } \quad y=\left(y_{1}, \ldots, y_{n}\right)\right.
$$

den Güterangebots-bzw. Faktormengenvektor im aggregierten Produktionssektor darstellt. Unter der Voraussetzung, daB alle Unternehmen Gewinnmaximierer sind und auf allen Märkten vollkommener Wettbewerb herrscht, kann man von der Fiktion eines repräsentativen Unternehmens ausgehen, das die aggregierten Gewinne $\sum_{i} p_{i} y_{i} \geqq 0$ bei gegebener Transformationsfunktion (II.17)zu maximieren sucht ${ }^{2}$ ).

Der Einfachheit halber werden im folgenden eine lineare Produktionstechnologie und damit konstante Produzentenpreise unterstellt, so daß die Gewinne langfristig null werden.

$$
\text { (II.19) } \quad \sum_{i} p_{i} y_{i}=0 \quad i=1, \ldots, n
$$

1) Vgl. zu diesem Problemkreis z.B. Dasgupta/Stiglitz (1972), Diamond/Mirrlees (1971a und b), Mirrlees (1972) oder Hahn (1973)

2) Der private Produktionsbereich muß unter den genannten Voraussetzungen immer auf der Transformationsfunktion produzieren. Die in dem Unternehmen eingesetzten Faktoren seien wiederum negativ definiert. 
Die Annahme konstanter Produzentenpreise ${ }^{1)}$ ist deshalb notwendig, weil sich bei der weniger restriktiven Annahme konstanter Skalenerträge nur mengenwertige Angebotsfunktionen ableiten lassen, d.h., daß der gegebene Preisvektor in mehrere Mengenvektoren abgebildet wird ${ }^{2}$. Konstante Produzentenpreise garantieren außerdem, daß das repräsentative Unternehmen seine produzierten Mengen permanent an die Marktnachfrage anpaßt. Das Reaktionsmuster des repräsentativen Produzenten ist wie im Falle der Konsumenten durch die Annahme eines einzigen Zieles gekennzeichnet, nämlich Gewinnmaximierung. Eingriffe in den politischen Entscheidungsproze $\beta$ finden nicht statt.

\section{II.2.3. Der Regierungssektor}

In der vorliegenden Modellökonomie erfüllt der Staat die klassische Aufgabe der unentgeltichen Bereitstellung eines Bündels öffentlicher Güter ${ }^{3)} \bar{z}$, das aufgrund eines nicht näher beschriebenen Entscheidungsprozesses als gesellschaftlich wünschenswert erachtet wird. Die Vernachlässigung kollektiver Entscheidungen

1) Die Annahme konstanter Produzentenpreise impliziert konstante Skalenerträge. Sofern man abnehmende Skalenerträge zuläßt, können aus

$L=\sum p_{i} x_{i}-\gamma[F(y)], i=1, \ldots, n$, die bekannten Güterangebots - und Faktornachfragefunktionen $y_{i}(p)$ der Unternehmen abgeleitet werden, die homogen vom Grade null in $p$ sind. Durch Einsetzen von $y_{j}(p)$ in (II.18) folgt die Gewinnfunktion $\pi(p)=\sum p_{i} y_{i}(p)>0$, die das Pendant zur Ausgabenfunktion darstellt. Bei abnehmenden Skalenerträgen können also Gewinne entstehen, die den Haushalten als Pauschaleinkommen zufließen. Die hier abgeleiteten Modellergebnisse, aber auch die Art der Normierung ändern sich nicht, wenn die positiven Gewinne (die Pauschaleinkommen der Haushalte) zu $100 \%$ besteuert werden, und wenn das Steueraufkommen niedriger liegt als das vom Staat geplante. Im allgemeinen wird es jedoch notwendig sein, alle Variablen in Abhängigkeit von den Produzentenpreisen zu schreiben. In diesen Fällen darf jedoch nur noch ein Preis normiert werden. Vgl. dazu Z.B. Munk (1978)

2) Vgl. dazu Wiegard (1978), S. 21 oder Tresch (1981), S. 70. Das Problem mengenwertiger Funktionen könnte allerdings dadurch umgangen werden, da $B$ man mit der inversen Angebotsfunktion arbeitet und statt der Preise und des Pauschale inkommens die Mengen als Instrumentenvariablen wählt. Vgl. zu dieser Vorgehenswe ise Wiegard (1976)

3) Im Sinne von Samuelson (1954) 
ist nicht nur die logische Konsequenz des Ein-Konsumenten-Modells, sondern beruht vor allem auf der politischen Abstinenz der Wirtschaftssubjekte ${ }^{1)}$. In diesem Fall wird daher unterstellt, daB die Optimierung des Kollektivgüterangebots durch einen allwissenden Planer erfolgt, der die Präferenzen der besteuerten Individuen kennt. Abweichungen von den optimalen Gütermengen haben zwar keinen direkten EinfluB auf die Optimierung der Finanzierungsseite bzw. Steuerstruktur, aber sie reduzieren das Nutzenniveau des repräsentativen Individuums. Wenn daher in einigen OT-Modellen von der Steuerfinanzierung irgendwelcher staatlicher Ausgaben ${ }^{2)}$ die Rede ist, muß man sich vergegenwärtigen, daß diese nicht zwingend im Interesse des Steuerzahlers liegen, da letztlich nur die Struktur der Steuersätze, nicht aber deren Höhe optimiert wird. Die Bedeutung der Ausgabenniveaus für Steuerreformempfehlungen wird noch ausführlich diskutiert, wenn alternative Verhaltensweisen des Fiskus in das Modell eingehen. Aufgrund der exogenen Fixierung der Budgethöhe ist aber ein umfassender optimierungsansatz nicht möglich; insofern handelt es sich hier um ein Partialmodell staatlicher Aktivitäten. Mit Blick auf diesen Vorbehalt ist die gedankliche Trennung von Ausgaben- und Einnahmeseite des staatlichen Budgets durchaus fruchtbar, weil die simultane Quantifizierung des optimalen Kollektivguterniveaus und eines optimalen steuersystems nur schwer möglich ist.

Es sei ferner angenommen, $d a \beta$ auch die Produktion von $\bar{z}$ im Staatssektor erfolgt, wobei nicht zwingend ein effizienter Faktoreinsatz vorausgesetzt wird: $\bar{z}=z\left(y_{i}^{z}\right)$. Es sei lediglich angenommen, da $B$ die staatlichen Produktionseinheiten ohne Gewinn oder Verlust arbeiten. D.h. die im privaten Haushaltsbereich gekauften Faktormen-

1) Die Hypothesen über die Zielsetzungen und Reaktionsmuster der Wirtschaftssubjekte im Mehr-Personen-Mode11 unterscheiden sich nicht von jenen in Abschnitt II.2.1.

2) Vgl. Z.B. Munk (1978), S. 3 "The Government needs fixed quantities of a number of commodities for some unspecified purposes..." Inwieweit ein Steuersystem als rational bezeichnet werden kann, das zwar die Mehrbelastung minimiert, aber Steuern für suboptimale Ausgaben erhebt, soll hier nicht weiter erörtert werden. 
gen $y_{j}^{z}$ werden über einen kostendeckenden Preis $p_{z}$ für $z$ dem Verwaltungsapparat in Rechnung gestellt1):

(II.20) $\quad p_{z} \bar{z}=-\sum_{i} p_{i} y_{i}^{z}$.

Ober die in der Produktion eingesetzten Faktormengen hinaus werden vom Fiskus zur Bereitstellung von $z$ (darunter kann man z.B. auch die statliche Verwaltungstätigkeit verstehen) weitere fix vorgegebene Güter- und Faktormengen $y_{j}^{g}$ von den privaten Haushalten benötigt, die er zu Marktoreisen erwerben muß. Zur Erfüllung seiner Allokations- (und Distributions-)aufgaben erhebt der Fiskus Güter- und Faktorstevern:

(II.21) $\quad t_{i}=q_{i}-p_{i}$.

AuBerdem kann er Pauschalsteuern bzw. -transfers ${ }^{2}$ m einsetzen, sofern keine expliziten Restriktionen bezüglich der Steuerstruktur existieren. Die Budgetrestriktion des Staates lautet daher

$$
\sum_{i} t_{i} x_{i}^{c}-m=-\sum_{i} p_{i} y_{i}^{g}+p_{z} \bar{z}=\bar{T}^{c} .
$$

\section{II.2.4. Die Marktgleichgewichtsbedingungen}

Im Gleichgewicht müssen die angebotenen und nachgefragten Mengen auf allen Märkten übereinstimmen, so daß unter Berücksichtigung von (II.3), (II.18) und den von statlicher Seite in fixem Ausmaß nachgefragten Inputmengen $y_{i}^{g}, y_{i}^{z}$ folgt:

1) Wiederum seien Inputs $y_{i}^{z}$ negativ definiert.

2) Sofern es sich um Pauschalsteuern hande1t, ist m negativ definiert. Im Falle von Pauschaltransfers positiv. 
(II.23) $\left.\quad x_{i}(q, \bar{u})=y_{i}+y_{i}^{g}+y_{i}^{z} \quad i=2, \ldots, n^{1}\right)$

Unter diesen Bedingungen lassen sich alle endogenen Größen des Modells in Abhängigkeit von $q$ und u darstellen; sie werden damit zu Kontrollvariablen der Regierung. Man kann diese Tatsache auch durch das totale Differential von Gleichung (II.21) deutlich machen:

(I I.24) $\quad d t_{i}=d q_{i}-d p_{i}=d q_{j}$;

d.h. die Regierung determiniert durch die Steuersätze die Konsumentenpreise.

\subsubsection{Das Walras-Theorem}

Werden alle Budgetrestriktionen von den Wirtschaftssubjekten erfüllt, und sind alle Märkte außer einem im Gleichgewicht, dann befindet sich auch dieser letzte Markt im Gleichgewicht. Dies impliziert auch umgekehrt: Wenn alle Märkte im Gleichgewicht sind und alle Wirtschaftssubjekte außer einem ihre Budgetgleichung erfüllen, dann ist auch die Budgetgleichung der letzten Einheit er$f u \dot{u} 11 t^{2)}$.

Die Feststellung bedeutet, daß man z.B. aus allen anderen Budgetrestriktionen und den Marktgleichgewichtsbedingungen die Budgetrestriktion des States oder eines anderen Aktors dieser okonomie ableiten kann. Durch Einsetzen von (II.21) und (II.23) in die Ausgabenfunktion des Haushalts und Addition der Budgets von Unterneh-

1) Da es sich um ein Modell mit kompensierten Marktgleichgewichten handelt, muß auf einem Markt für die notwendigen Kompensationen gesorgt werden, so da $B$ lediglich $n-1$ Märkte geräumt sein müssen. Vgl. zu dieser Problematik Tresch (1981), S. 78 f. Setzt man, wie oben erwähnt, konstante Produzentenpreise voraus, so passen sich die angebotenen Güter- und Faktormengen grundsätzlich an die Nachfrageseite an; die explizite Modellformulierung wurde jedoch gewählt, um ohne größeren Aufwand auch Modelle mit abnehmenden Skalenerträgen berücksichtigen zu können.

2) Vgl. Diamond/Mirrlees (1971a), S. 14 
men und staticher Produktionsstäte folgt:

(II.25)

$$
\sum_{i}\left(p_{i}+t_{i}\right)\left(y_{i}+y_{i}^{g}+y_{i}^{z}\right)=m+\sum_{i} p_{i} y_{i}+\sum_{i} p_{i} y_{i}^{z}+p_{z} \bar{z}
$$

bzw.

$$
\sum_{i} t_{i} x_{i}^{c}+\sum_{i} p_{i} y_{i}^{g}=m+p_{z} \bar{z} .
$$

Damit resultiert auch die statiche Budgetrestriktion (II.22).

\section{II.2.6. Die Normierung}

Die abgeleiteten Angebotsfunktionen und die kompensierten Nachfragefunktionen sind homogen vom Grade null in den Produzentenbzw. Konsumentenpreisen. Multipliziert man die Produzenten- bzw. Konsumentenpreise mit jeweils einer Konstanten, so ändern sich die angebotenen bzw. nachgefragten Güter - und Faktormengen nicht. Wählt man eine Konstante $k>0$ zur Multiplikation der Produzentenpreise derart, daß der Preis eines Inputs (z.B. Arbeit) eins wird, und wählt man eine geeignete Konstante $k^{\prime}>0$, so daB der Preis des Faktors Arbeit im Konsumentenpreisvektor ebenfalls eins wird, dann bedeutet dies, daß Arbeit das Numérairegut darstellt und wegen $p_{1}=q_{1}=1$ nicht besteuert würde. Im Falle konstanter Skalenerträge ${ }^{1)}$ ist dies die gebräuchlichste Normierung in der OTLiteratur; die Fixierung eines Produzenten- und eines Konsumentenpreises ist gleichzeitig eine notwendige Voraussetzung für eine eindeutige Lösung dieses allgemeinen Gleichgewichtsmodells ${ }^{2}$ ). Je nach Untersuchungsziel ist es allerdings möglich, jedes andere Gut im Modell als numéraire zu wählen, ohne daß das systemgleich-

1) In diesem Modell werden nicht nur konstante Skalenerträge, sondern zusätzlich konstante Produzentenpreise vorausgesetzt.

2) Vgl. dazu Munk (1978), S. 3 ff. 
gewicht davon beeinflußt würde ${ }^{1)}$. Im Gegensatz zu den kompensierten Nachfragefunktionen sind die Marktnachfragefunktionen homogen vom Grade null in Preisen und Einkommen ${ }^{2)}$, so daB der Einkommensbestandteil $m$ in der Budgetrestriktion bzw. Nachfragefunktion ebenfalls mit der Konstanten multipliziert werden muB, wenn die nachgefragten Mengen und damit das Systemgleichgewicht unverändert bleiben sollen; m bzw. ein Vielfaches davon wird somit in der Budgetrestriktion bzw. uiber die Marktnachfragefunktion automatisch berücksichtigt. Wählt man dagegen den dualen Optimierungsansatz, so muß der in den Hicks'schen Nachfragefunktionen unberücksichtigte fixe Einkommensbestandteil in einer zusätzlichen Restriktion erfaßt werden. Im allgemeinen Gleichgewicht muß nämlich gelten, daß m genau den minimalen Ausgaben nach Steuern (beim Gleichgewichtspreisvektor $q$ und dem zu maximierenden Nutzenniveau u) entspricht:

(II.26) $\quad m=e(q, u)$.

Der numerische Wert der Ausgabenfunktion ergibt die Größe von m in der Budgetrestriktion (II.2), wenn das in (II.7) fixierte Nutzenniveau dem aus dem primalen optimierungsproblem maximal erreichbaren Nutzenniveau entspricht.

\section{II.2.7. Das staatliche Optimierungsproblem}

Die Struktur eines rationalen Steuersystems kann dadurch bestimmt werden, daß die Regierung das Nutzenniveau des repräsentativen Konsumenten maximiert, wobei die Budgetrestriktion des Wirtschaftssubjekts und die des Staates die Nebenbedingungen des Optimierungsansatzes ${ }^{3)}$ darstellen:

1) Zur Normierung allgemeiner Gleichgewichtsmodelle stehen neben der oben genannten Möglichkeit noch andere Normierungsarten zur Verfügung. Vgl. dazu Munk (1978), S. $3 \mathrm{ff}$. Oder Munk (1980), S. 759

2) $\mathrm{Vgl}$. Henderson/Quandt (1983), S. $19 \mathrm{f}$.

3) Der bekannte alternative Optimierungsansatz mit $\mathrm{Hilfe}$ der indirekten Nutzenfunktion wird im Anhang A dargestel1t. Eine ausführliche Interpretation findet sich Z.B. bei Sandmo (1976) oder Atkinson/Stiglitz (1980), Kap. 12 und 14. $\mathrm{Vgl}$. zu einem Optimierungsansatz mit zunehmenden Skalenerträgen Munk (1978). 
(II.27)

$$
\begin{aligned}
L & =u+\mu[m-e(q, u)]+ \\
& +\theta\left\{\sum_{i=2}^{n} t_{i} x_{i}(q, u)+\sum_{i=1}^{n} p_{i} y_{i}^{g}-m-p_{z} \bar{z}\right\}
\end{aligned}
$$

mit $t_{1}=0$, wenn das erste Gut nicht besteuert wird ${ }^{1)}$. Die Bedingungen erster Ordnung erhält man durch partielles Differenzieren von (II.27) nach allen Kontrollvariablendes Staates (q), dem Maximanden $(u)$ und den Lagrange-Multiplikatoren $(\mu, \theta)$, wobei allerdings $d_{q}=0$ berücksichtigt werden muß:

(II.28) $\quad \frac{\partial L}{\partial q_{k}}=-\mu \frac{\partial e}{\partial q_{k}}+\theta \sum_{i} t_{i} \frac{\partial x_{i}^{c}}{\partial q_{k}}+\theta x_{k}^{c}=0$

mit $i=2, \ldots, n$ und $k=2, \ldots, n$

(II.29) $\frac{\partial L}{\partial m}=\mu-\theta=0$

(II.30) $\quad \frac{\partial L}{\partial u}=1-\mu \frac{\partial e}{\partial u}+\sum_{i} t_{i} \frac{\partial x_{i}^{c}}{\partial u}=0$

(II.31) $\quad \frac{\partial L}{\partial \mu}=m-e(q, u)=0$

(II.32) $\quad \frac{\partial L}{\partial \theta}=\sum_{i} t_{i} x_{i}(q, u)+\sum_{i} p_{i} y_{i}^{g}-m-p_{z} \bar{z}=0$

II.3. Steuerregeln für optimale Steuersysteme

Am Beispiel der folgenden OT-Regeln sollen vor allem die Wirkungen von alternativen steuerpolitischen Restriktionen, d.h. Begrenzungen der Steuerinstrumente, der Steuerbemessungsgrundlagen sowie

1) Daher laufen die Indizes $i, k$ in den folgenden Gleichungen von $2, \ldots, n$, sofern nicht explizit auf Anderungen hingewiesen wird. Die Summenzeichen sind entsprechend zu lesen: $\sum_{i, k=2}^{n}$ 
des individuellen Nachfrageverhaltens auf die optimale Steuerstruktur verdeutlicht werden. Die Analyse belegt, daB bereits geringfügige Anderungen der Modellannahmen zu erheblichen Konsequenzen für die OT-Empfehlungen führen können.

\section{II.3.1. First-Best-Lösungen und Pauschalsteuern}

Zunächst kann das bekannte Ergebnis abgeleitet werden, daß die ausschlieBliche Erhebung von Pauschalsteuern optimal wäre ${ }^{1)}$. Unter Berücksichtigung von (II.13) und (II.16) in (II.28) folgt:

(II.33) $\quad \sum_{i} t_{i} s_{i k}=\frac{-(\theta-\mu)}{\theta} x_{k}^{c}$

b zw.

(II.34) $\quad \sum_{i} t_{i} s_{i k}=k x_{k}^{c}$ oder

$$
\frac{\sum_{i} t_{i} s_{i k}}{x_{k}^{c}}=k \quad \text { mit } k=-\frac{(\theta-\mu)}{\theta} \text {, }
$$

wobei $k$ negativ ${ }^{2}$ ) und unabhängig von der linken Seite in (II.35) ist. Genau dasselbe Ergebnis erhält man durch die Minimierung der Mehrbelastung unter der Nebenbedingung eines gegebenen Steueraufkommens ${ }^{3)}$. Als Mehrbelastung oder excess burden bezeichnet man jene Nutzenverluste, die über die reine Steuerzahlung hinaus durch die Verzerrung der relativen Preise und den daraus resultierenden Substitutionseffekten verursacht werden. Die Minimierung der Mehrbelastung ist deshalb auch eine alternative Formulierung der Opti-

1) Vgl. Z.B. Atkinson/Stiglitz (1980), S. 429

2) Der Nachweis dieser Behauptung sowie die Interpretation der hier verwendeten Lagrange-Multiplikatoren finden sich in Anhang $A$.

3) Vgl. dazu Anhang B. 
mierungsaufgabe (II.27). Setzt man (II.29) in (II.33) ein, folgt, daß alle Güter- und Faktorsteuerbeträge im Optimum gleich null sein müssen. Es resultiert die

\section{OT-Rege 1 1:}

Um ein First-Best-0ptimum zu erreichen, muß der Fiskus $\bar{T}^{c}$ ausschließlich durch Pauschalsteuern finanzieren.

Weil die Slutsky-Matrix negativ definit und ihre Determinante daher ungleich null ist, gibt es für alle $t(t=0)$ eine eindeutige Lösung. Der Vorzug einer Pauschalsteuer beruht auf ihrer Neutralität gegenüber dem System relativer Preise, so daB keine Substitutionseffekte entstehen, die für Nutzeneinbußen, genauer für die Mehrbelastung, konstitutiv sind. Das Ergebnis erscheint recht einfach; es hat jedoch weitreichende, über dieses Ein-Konsumenten-Modell hinausgehende Konsequenzen, denn Pauschalsteuern $(m<0)$ sind sowohl Charakteristika des hier konzipierten Modells, als auch das Kennzeichen der direkten Besteuerung ${ }^{1}$. Das Effizienzziel sollte daher der direkten Besteuerung vorbehalten sein ${ }^{2}$. Dies steht im Gegensatz zu vielen traditionellen ökonomischen Vorstellungen ${ }^{3)}$. Innerhalb dieser Modellökonomie, die lediglich die effizienteste Steuerlösung zum Ziel hat, muß konsequenterweise eine Pauschalsteuer erhoben werden. Die beste Steuer wäre hier tatsächlich eine einheitliche Kopfsteuer auf alle identischen Individuen, so daß Probleme einer differenzierten Pauschalbesteuerung gar nicht auftreten. Warum aber, so muB man sich fragen, ist diese einfache Art der Besteuerung in der aktuellen Steuergesetzgebung und in der politischen Diskussion ohne Bedeutung? Warum stellt man statt dessen fest, $d a B$ es in beinahe allen existierenden Einkommensteuersystemen irgendwelche Freibeträge und damit Pauschaltransferelemente gibt?

1) Vgl. Anhang $C$ oder zu einer ausführlichen Interpretation Atkinson (1977), S. $590 \mathrm{ff}$.

2) Vgl. zu diesem Ergebnis auch ein Mehr-Personen-Modell, Atkinson/Stiglitz $(1980)$, S. 432

3) Vgl. Z.B. Fromm/Taubman (1973), S. 139 
Diese Fragen können natürlich nur modellexogen beantwortet werden, beispielsweise mit dem Hinweis darauf, daB hier Verteilungsgesichtspunkte vernachlässigt werden. Speziell auf dieses Argument wird im Zusammenhang mit dem Mehr-Personen-Modell eingegangen. Allerdings kann gezeigt werden, daß der Verteilungsaspekt allein kein ausreichendes Argument gegen Pauschalsteuern sein kann. Vielmehr steht zu vermuten, daß die außerordentlich einfache Pauschalsteuer auch politisch nicht durchsetzbar sein wird, weil ihre Einführung ohne die Berücksichtigung der ökonomischen Verhältnisse des Steuerzahlers auf den Widerstand der Bevölkerung stoßen würde. Zunächst sei von der Tatsache ausgegangen, daß das gesamte Steueraufkommen mittels Pauschalsteuern nicht finanziert werden kann, da dies einen zu radikalen Eingriff in das bestehende Steuersystem bedeuten würde. Die Begrenzung oder das Verbot von Pauschalsteuern entsprechen einer Restriktion des Steuerinstrumentariums und machen das staatliche Optimierungsproblem zu einem Second-Best-Problem. In den folgenden Abschnitten soll überprüft werden, wie die OT-Regeln unter diesen Bedingungen modifiziert werden müssen.

\section{II.3.2. Second-Best-Steuerrege In}

Damit man Second-Best-Regeln ableiten kann, muß die oben angeführte Möglichkeit der Pauschalbesteuerung entweder entfallen $(m=0)$ oder aber so eingeschränkt werden, daß $\bar{T}$ nicht voll über die maximal erzielbaren Pauschalsteuern finanziert werden kann $(m=\bar{m}<\bar{T})$. Durch diese Einschränkungen des Steuerinstrumentariums erhält man die bekannte Pigou'sche Problemstellung, die Ramsey ${ }^{1)} 1927$ untersuchte. Ferner wird wiederum unterstellt, da $B$ die Regierung ein bestimmtes Steueraufkommen erzielen will. Mit $m=0$ wird der Wert der Ausgabenfunktion (II.12) gleich null und

1) Vg1. dazu Ramsey (1927). "The problem I propose to tackle is this: given revenue is to be raised by proportionate taxes on some or all uses of income, the taxes on different uses being nossibly at different rates; how should these rates be adjusted in order that the decrement of utility may be a minimum; [...] Nevertheless we shall find that the obvious solution that there should be no differentiation is entirely erroneous." Ramsey (1927), S. 47 
$m$ verschwindet in der staatlichen Budgetrestriktion. In jedem Fall ( $m=\bar{m}$ oder $m=0$, d.h. ohne Variationsmöglichkeit der Pauschalsteuern) kann die_optimale Güter- und Faktorsteuerstruktur, die notwendig ist, um $T$ teilweise oder ganz zu finanzieren, aus (II.28) abgeleitet werden. Da Pauschalsteuern nicht mehr erhoben werden durfen, handelt es sich im folgenden um Second-Best-oder Feasible-First-Best-Model1 $e^{1 \text { ) }}$.

\section{I.3.2.1. Die Ramsey-Rege I}

Gleichung (II.35) erlaubt die Charakterisierung optimaler Steuerstrukturen durch die bekannte Ramsey-Steuerregel.

Der Zähler gibt näherungsweise die gesamte Mengenänderung der Güter und Faktoren an, die aufgrund aller Steuerbetragsvarianten erfolgen:

$$
\Delta x_{k}^{c}=\sum_{i=2}^{n} t_{i} \cdot \frac{\Delta x_{k}^{c}}{\Delta t_{i}}=\sum_{i=2}^{n} \Delta q_{i} \frac{\Delta x_{k}^{c}}{\Delta q_{i}}
$$

Die relative Mengenänderung $\Delta x_{k}^{C} / x_{k}$ muß dann gemäß (II.35) jeweils der Größe k entsprechen. Es gilt somit:

\section{OT-Regel 2:}

Die Ramsey-Rege 12) [Gleichung (II.35)] charakterisiert ein Optimum derart, daß eine gleiche prozentuale Anderung aller Steuerbeträge pro Mengeneinheit $z u$ einer gleichen prozentualen Anderung der Gleichgewichtsmengen aller besteuerten Güter und Faktoren

1) Vgl. Samuelson (1958), S. 540

2) Zur Ramsey-Regel siehe z.B. Tresch (1981), S. 326 f., Rose/Wenzel/Wiegard (1981), S. 76, Atkinson/Stiglitz (1980), S. 373. Diese Regel gilt auch für ein beliebig großes Steueraufkommen. Der Aussagegehalt des ursprünglichen Theorems wird jedoch dadurch eingeschränkt, daß dt $=t$ gilt und die Steuerbeträge pro Mengeneinheit gegen null gehen, so daß auch das zu erzielende Steueraufkommen ganz gering sein muß. Vgl. Z.B. Wiegard (1976), S. 205 oder Mirrlees (1975), S. 27, Ramsey (1927), S. 47, Krause-Junk/v. Oehsen (1981) und insbesondere Green (1975), S. 370 
führen muß, wobei der Konsument so kompensiert wird, daß er sein ursprüngliches Nutzenniveau realisiert.

Leider gibt die Ramsey-Regel nicht direkt die relative Höhe der einzelnen Steuersätze an, sondern beschreibt die Wirkung eines optimalen Steuersystems auf prozentuale Nachfrageänderungen. Dies bedeutet, daß man die optimale Struktur von vornherein zumindest approximativ kennen müßte, um ein Optimum zu garantieren. Anders ausgedrückt: Mit Hilfe der Ramsey-Regel kann (in der Tendenz) nur überprüft werden, "ob ein existierendes Steuersystem optimal ist"1) Ebenso kann a priori nicht festqestellt werden. ob ein einheitliches oder differenziertes Steuersystem optimal ist. Es ?äßt sich jedoch schon jetzt vermuten, daß die Anhebung aller Steuerbeträge um einen einheitlichen Prozentsatz $\left(d . h \cdot d t_{i}=d t\right)$ nur in Ausnahmefällen zu einer prozentual gleichen Reduktion der Gleichgewichtsmengen der besteuerten Güter und Faktoren führt. Allerdings kann man sofort sehen, daß aus einer Begrenzung von Pauschalsteuern $(0<\overline{\mathrm{m}}<\overline{\mathrm{T}})$ erhebliche Änderungen beim Einsatz des übrigen Steuerinstrumentariums resultieren. Die Ramsey-Regel erlaubt keine zusätzlichen intuitiven Einsichten in die optimale Steuerstruktur, so daB es sinnvoll erscheint, weitere ökonomische Implikationen der OT zu illustrieren. Als Beispiel wird zunächst das Problem von Corlett und Hague behandelt.

\section{II.3.2.2. Die Corlett-Hague-Rege ${ }^{2 \text { ) }}$}

Es sei Arbeit $\left(x_{1}\right)$ der einzige Faktor und die Zahl der Güter sei auf $x_{2}$ und $x_{3}$ beschränkt. Nach einigen Umformungen erhält man aus ( I I . 34):

(II.37) $\frac{t_{2}}{q_{2}}=\tau_{2}=k^{\prime}\left(\sigma_{22}+\sigma_{33}+\sigma_{21}\right)$

1) Sandmo (1976)

2) Vgl. Corlett/Hague $(1953 / 54)$, S. $21 \mathrm{ff}$. oder zu einer einfacheren Interpretation Sandmo (1976), S. 46 


$$
\frac{t_{3}}{q_{3}}=\tau_{3}=k^{\prime}\left(\sigma_{22}+\sigma_{33}+\sigma_{31}\right)
$$

sowie

(II.38) $\tau_{2}>\tau_{3}$, wenn $\sigma_{31}<\sigma_{21}$

wobei $\tau_{i}=t_{i} / p_{i}$ die auf den jeweiligen Konsumentenpreis bezogenen Steuersätze, $\sigma_{22}$ bzw. $\sigma_{33}$ die kompensierten Preiselastizitäten der Güter 2 bzw. 3 und $\sigma_{21}$ bzw. $\sigma_{31}$ die kompensierten Kreuzpreiselastizitäten zwischen den Gütern 2 bzw. 3 und Arbeit sind. Aus (II.37) folgt:

OT-Rege $13:$

Aus (II.37) bzw. (II.38) ergibt sich, daB im Optimum das Gut mit der geringeren kompensierten Kreuzpreiselastizität der Nachfrage in Bezug auf den Preis für Arbeit am höchsten besteuert werden sol1. Dies impliziert, daB das Gut mit der stärksten Komplementaritätsbeziehung zur Freizeit am höchsten besteuert werden muB ${ }^{1}$ ). Die Steuerregel wird intuitiv einsichtig, wenn man Freizeit als Gut definiert, das nicht besteuert werden kānn. Die Steuer auf das Komplementärgut wirkt jedoch wie eine indirekte steuer und verzerrt daher das relative Preisgefüge weniger stark, so daß die für die Mehrbelastung konstitutiven Substitutionseffekte nur in kleinerem Umfang auftreten ${ }^{2}$ ).

Eine Verallgemeinerung der intuitiven ökonomischen Interpretation

1) Daher läßt sich auch vermuten, daß die Erhöhung einheitlicher Mehrwertsteuersätze, gemessen am Pareto-Kriterium suboptimal ist.

2) Diese Oberlegungen könnten nun den Schluß nahelegen, alle Güter inklusive Freizeit mit einem einheitlichen Steuersatz zu besteuern, um das relative Preisgefüge zu erhalten und daher keine Substitutionseffekte aus der Besteuerung zu verursachen. Die Folge wäre allerdings e in Steueraufkommen von nul1, sofern die Regierung keine anderen Einnahmen zur Verfijgung stehen. Vgl. hierzu beispielsweise Sandmo (1974), S. 702. Zum Problem direkte versus indirekte Steuern siehe auch Little (1951). 
leitet Boadway in einem modifizierten Modellansatz ab: "... When some goods are untaxable, the tax rates for the remaining goods must be increased more for those goods which are most complementary for those goods one would most like to tax, but cannot"1). Die Corlett-Hague-Regel macht die Abhängigkeit der OT-Regeln von den individuellen Substitutionsreaktionen der Individuen besonders deutlich. Um die Steuersätze zu quantifizieren, müßten alle kompensierten Kreuzpreiselastizitäten geschätzt werden. Auf die Problematik der empirischen Ermittlung dieser Daten wird noch ausführlich eingegangen.

\section{II.3.2.3. Proportionale Steuerregeln}

Die steuerpolitische Bedeutung der kompensierten Nachfragereaktionen auf Anderungen der relativen Preise wird offenkundig, wenn man unterstellt, die kompensierten Kreuzpreiselastizitäten $\tau_{21}$ und $\tau_{31}$ seien gleich. Aus (II.51) folgt sofort $\tau_{2}=\tau_{3}$, d.h. eine einheit1 iche Gütersteuer wäre optimal ${ }^{2}$ ).

Unter der Voraussetzung eines vollkommen unelastischen Arbeitsangebots erhält man dasselbe Ergebnis. Zu diesem Zweck wird (II.12) nach $q_{k}$ differenziert:

$$
x_{k}^{c}+\sum_{i} q_{i} \frac{\partial x_{i}^{c}}{\partial q_{k}}+\frac{\partial x_{1}^{c}}{\partial q_{k}}=0
$$

1) "This constitutes a generalization of the Corlett and Hague theorem in which only one untaxed good exists", Boadway (1975), S. 282. Ein Ansatz, der die indirekte Nutzenfunktion verwendet, findet sich bei Stiglitz/Dasgupta (1971); die Ergebnisse sind jedoch schwerer zu interpretieren.

2) Dieses Ergebnis läßt sich verallgemeinern. Es gilt immer bei homothetischen Nutzenfunktionen, die schwach separabel zwischen Arbeit und den besteuerten Gütern (Faktoren) sind. Vgl. zu separablen Nutzenfunktionen Goldman/Uzawa (1964), S. $387 \mathrm{ff}$. Homothetische Nutzenfunktionen haben die Eigenschaft, daß die Nachfrage aller Güter um den gleichen Prozentsatz steigt, wenn das Einkommen prozentual erhöht wird. "Ein solches Verhalten stimmt mit unseren empirischen Beobachtungen aber nicht überein". Rose/Wiegard (1983), S. 50 
und in (II.34) eingesetzt:

(II.40) $\quad \sum_{i=2}^{n}\left(t_{i}-k q_{i}\right) s_{i k}=0$.

Dies ergibt

OT-Rege 1 :

Wenn die Mengen des unbesteuerten Gutes (z.B. Freizeit) $x_{1}$ auf Preisvariationen der besteuerbaren Güter und Faktoren nicht reagieren, ist eine proportionale Steuerstruktur $\left(t_{i}=k q_{j}\right)$ optimal ${ }^{1}$. Das Ergebnis gilt dann, wenn der letzte Term in (II.39) null wird, d.h. wenn die Nachfrage nach Freizeit (gegenüber allen anderen Konsumentenpreisen) völlig unelastisch ist.

Dies wird auch intuitiv einsichtig, wenn man bedenkt, daß ein proportionaler Steuersatz $t$ auf alle Güter und Faktoren mit Ausnahme von z.B. Arbeit genau einer alleinigen Besteuerung dieses Faktors Arbeit zum Steuersatz $t$ ' entspricht. Das völlig unelastische Arbeitsangebot ist aber eine ideale Steuerbemessungsgrundlage im Sinne der OT, da eine Steuer auf das konstante Arbeitsangebot keine Substitutionseffekte verursacht und daher in ihrer Wirkung einer Pauschalsteuer entspricht.

Die ökonomische Relevanz von Steuersystemen mit einheitlichem Steuersatz ist äußerst gering, weil die Voraussetzungen mit den empirischen Beobachtungen nicht übereinstimmen. Abgesehen von der empirischen Evidenz könnten beispielsweise allgemeine Verbrauchsteuern schon wegen steuerlicher Restriktionen bzw. institutioneller Hemmnisse nicht zu empfehlen sein. Werden einige Güter und Faktoren aus modellexogenen Gründen nicht bestevert $\left(z, B, x_{j}, j=m+1, \ldots, n\right)$, so erhält man aus (II.12) statt (II.39):

1) Vgl. z.B. Dixit (1970), S. $196 \mathrm{ff}$. 
(II.41) $x_{k}^{c}+\sum_{i=2}^{m} q_{i} \frac{\partial x_{i}^{c}}{\partial q_{k}}+\sum_{j=m+1}^{n} q_{j} \frac{\partial x_{j}^{c}}{\partial q_{k}}+q_{1} \frac{\partial x_{1}^{c}}{\partial q_{k}}=0$

und statt (II.40):

(II.42) $\quad \sum_{i=2}^{m}\left(t_{i}-k q_{j}\right) s_{i k}=k \sum_{j=m+1}^{n} q_{j} s_{j k}$, mit $s_{1 k}=0$,

d.h. eine proportionale Steuerstruktur wird in der Regel nicht mehr optimal sein. Das Ergebnis veranschaulicht wiederum die Bedeutung der Restriktion des Steuerinstrumentariums (Steuerbemessungsgrundlage oder Steuersätze) auf die Struktur eines optimalen Steuersystems. Wenn aus politischen Gründen der Faktor Arbeit und z.B. Grundnahrungsmittel nicht mehr besteuert werden duirfen, so kann der Steuerausfall nicht einfach durch die Erhöhung von bisher proportionalen Steuersätzen ausgeglichen werden; statt dessen muB die optimale Steuerstruktur völlig neu konzipiert werden, wobei wiederum die individuellen Preis-und Kreuzpreiseffekte, entsprechend der oben zitierten Boadway-Regel, die entscheidende Rolle spielen.

\section{II.3.2.4. Die inverse Elastizitätsregel}

Nun fehlen häufig die Informationen über die kompensierten Kreuzpreiselastizitäten oder aber sie werden der Einfachheit halber vernachlässigt $\left(s_{i k}=0\right)$. Wenn die Güternachfrage (Faktorangebot) nur noch vom eigenen Preis abhängt, läßt sich eine bekannte OTRegel ableiten, die wegen ihrer restriktiven Annahmen äußerst zurückhaltend interpretiert werden muß. Nimmt man an, daß alle Güter und Faktoren nur vom eigenen Preis abhängen, d.h. die Kreuzpreiseffekte null sind $\left(s_{i k}=0\right)$, so vereinfacht sich (II.34) zu

(II.43) $\quad t_{i} \frac{\partial x_{i}^{c}}{\partial q_{i}}=k x_{i}^{c}$ 
und nach Umformen folgt ${ }^{1}$ :

(I I.44) $\frac{t_{i}}{q_{i}}=\frac{k}{\varepsilon_{i}}$ mit $\varepsilon_{i}=\frac{\partial x_{i}}{\partial q_{i}} \frac{q_{i}}{x_{i}}$.

Dies ergibt

OT-Regel 5 :

Wenn alle Güter und Faktoren nur von ihrem eigenen Preis abhängig sind, dann sollten die Güter (Faktoren) mit der niedrigsten kompensierten Nachfrage- (Angebots-)elastizität am höchsten besteuert werden. Sind alle $\varepsilon_{i} g l e i c h$, so ist eine proportionale Steuerstruktur optimal.

Die inverse Elastizitätsreqel hat eine intuitive ökonomische Interpretation. Je unelastischer die Güternachfrage auf eine Steuererhöhung reagiert, um so kleiner bleibt das Mehrbelastungsdreieck unterhalb der Nachfragekurve 2 ). Die der inversen Elastizitätsregel zugrunde liegenden Annahmen sind allerdings recht unrealistisch, so daB sie kaum als politische Richtschnur dienen können ${ }^{3)}$. Trotz dieser etwas unbefriedigenden Erkenntnis erlauben die OT-Regeln 1 - 5, unabhängig von ihrer spezifischen Ausgestaltung, einige allgemeine Bemerkungen in Bezug auf optimale Steuerreformen.

\section{II.4. Allgemeine Grundsätze für optimale Steuerreformen}

Die bisherigen Oberlegungen zur Struktur optimaler Steuersysteme haben illustriert, daB die OT-Regeln äUBerst empfindlich auf Variationen des Steuerinstrumentariums und der zur Verfügung stehenden Informationen reagieren. Ober Beschränkungen der Steuerbemessungsgrundlage und der Steuersätze wird aber im politischen Pro$z e B$, und daher modellexogen, entschieden.

Die Beobachtung und Analyse des laufenden politischen Prozesses

1) Vgl. Z.B. Sandmo (1976), S. 45

2) $\mathrm{Vgl}$. Anhang $B$

3) Vgl. zur Kritik z.B. von Oehsen (1982), S. $102 \mathrm{ff}$. oder zur Modifikation der Regel Tresch (1981), S. 330 f. 
impliziert, daß sinnvolle Steuerreformempfehlungen nur im Rahmen politisch vorgegebener bzw. akzeptabler Steuerinstrumente erfolgreich sein können. Die wesentliche Aufgabe einer auch über längere Zeit hinweg optimalen Steuerstrukturanalyse und der darauf aufbauenden Steuerreformen besteht darin, langfristig akzeptable steuerpolitische Rahmenbedingungen zu identifizieren. Die Analyse des politischen Prozesses von Steuerreformen ist daher ein unverzichtbarer Bestandteil einer anwendungsorientierten optimalen Steuertheorie.

Ohne das Herausfiltern langfristig konstanter Rahmenbedingungen ist eine auf lange sicht angelegte Steuerreformstrategie undenkbar. Denn die vielfachen politischen Eingriffe in die Steuerstruktur müßten simultan von ebenso häufigen Umgestaltungen des Steuersystems begleitet werden, um ein optimales system zu garantieren. Solche permanenten und teilweise radikalen Kursänderungen sind jedoch weder ökonomisch erwünscht noch politisch durchsetzbar. Sie würden einen gigantischen Verwaltungsaufwand verursachen und individuelle ökonomische Entscheidungen mit einem unkalkulierbaren Risiko versehen. Bei jeder steuerreform sollte daher berücksichtigt werden:

Steuerreformregel 1:

Vor jeder Steuerstrukturänderung sollte ein möglichst umfassendes, langfristiges und politisch akzeptiertes Steuerinstrumentenpotential definiert werden. Dies impliziert einen Konsens über die Steuerbemessungsgrundlage und den Einsatz verschiedener Steuerarten (Pauschalsteuern, Gütersteuern, Faktorsteuern u.a.).

Langfristig akzeptable Steuerinstrumente hängen jedoch nicht nur von ihrer Kompatibilität mit dem politischen Prozeß ab, sondern auch davon, welche Informationen zur Realisierung einer optimalen Steuerstruktur notwendig sind, und welches historisch gewachsene Steuersystem vor der Reform existiert. Die steuerpolitische Bedeutung der historischen Komponenten wird nicht nur durch die Behauptung, daß alte Steuern gute Steuern seien ${ }^{1)}$, belegt, sondern

1) Vgl. zu dieser Carnard'schen Steuerregel Schmölders/Hansmeyer (1980), S. 50 
vor allem durch die Erfahrung, daß Steuerstrukturen nur in den seltensten Fällen radikal reformiert werden. Dies hat $z$ wei Gründe: Erstens brechen Politiker ganz allgemein nur ungern mit lieb gewordenen Traditionen, und zweitens wehren sich die durch die Reform unvorteilhaft getroffenen Individuen vehement gegen eine zu starke Lastumverteilung. Darüber hinaus werden endlich beschlossene Steueränderungen mit weitreichenden obergangsregelungen versehen, die gewährleisten, daß ökonomische Entscheidungen, die auf der alten Gesetzesgrundlage gefällt wurden, so revidiert werden können, daB möglichst geringe negative Auswirkungen für die Betroffenen entstehen.

In der vorangegangenen OT-Analyse blieb das Problem schrittweiser Reformen ausgeklammert. Es konnte nur geprüft werden, wann ein Optimum erreicht ist, ohne bezug auf die historischen Gegebenheiten und ohne Handlungsanweisungen für kleine Steuerreformen, die im politischen EntscheidungsprozeB tatsächlich eine Rolle spielen könnten. Im nächsten Abschnitt soll nun dargestel1t werden, daB die OT auf dem Gebiet der schrittweisen Steuerreformtheorie ein gutes Stück vorangekommen ist, daß andererseits aber alle Probleme der exogenen Informationsflüsse und Entscheidungsprozesse nach wie vor bestehen bleiben.

\section{II.5. Das Problem schrittweiser Steuerreformen ${ }^{1}$ )}

Green illustriert den Mangel der vorangegangenen OT-Analyse wie folgt: "A description of Canterbury Cathedral, no matter how detailed, is of little use to me if I am seeking directions to Canterbury from London"2). "Die für die aktuelle Steuerpolitik eigentlich relevante Frage lautet also: welche kleinen strukturellen Anderungen des im Ausgangsgleichgewicht realisierten Systems

1) Dieses Problem wurde erst in neuerer Vergangenheit von der Finanztheorie betrachtet. Als grundlegende Beiträge sind zu nennen: Dixit (1975), Dixit/ Munk (1977), Green (1975), sowie Wiegard (1980). Weiterfuihrende theoretische Abhandlungen finden sich bei Guesnerie (1977), Hatta (1977), Diewert (1978) und Weymark (1978), (1979), (1981), Dixit (1979), Tirole/Guesnerie (1981), Pazner/Sadka (1981).

2) Green (1975), S. 370 
[ von ] Steuern führen zu einer Verbesserung?" ${ }^{1]}$.

\section{II.5.1. Die Ableitung des Wohlfahrtskriteriums}

Zur Ableitung des Wohlfahrtskriteriums greift man auf die staatliche Budgetrestriktion und die Ausgabenfunktion des Haushaltes zurück, die alle modellrelevanten Beziehungen beinhalten. Dieses reduzierte Gleichungssystem erlaubt die Erfassung potentieller Steuerbetragsänderungen, wenn die Variation aller $t_{j}$ berücksichtigt wird. Das totale Differential der staatichen Budgetrestriktion

$$
\sum_{i=2}^{n} t_{i} \frac{\partial x_{i}^{c}}{\partial t_{k}} d t_{k}+x_{k}^{c} d t_{k}-d m=0 \quad k=2, \ldots, n
$$

bzw.

$$
\sum_{i=2}^{n} \sum_{k=2}^{n} t_{i} s_{i k} d t_{k}+\sum_{k=2}^{n} x_{k}^{c} d t_{k}-d m=0
$$

und das totale Differential der Ausgabenfunktion

(I I.47) $\quad \sum_{i=1}^{n} \frac{\partial e}{\partial q_{i}} d q_{i}=d m$

bzw.

$$
\sum_{i=1}^{n} x_{k}^{c} d q_{i}=d m \quad \text { mit } d q_{1}=d t_{1}=0
$$

beinhalten demnach alle Preisvariationen der Modellgleichungen. Durch Einsetzen von (II.48) in (II.46) sowie unter Berücksichtigung von $d t_{i}=d q_{i}$ erhält man das gesuchte Wohlfahrts-

1) Diese Fragestellung geht auf Wiegard (1980), S. 1 f. zurück. Wiegard behandelt allerdings nur Probleme der schrittweisen Verbesserung der Struktur indirekter Steuern. 
kriterium.

(II.49) $\quad d W=\sum_{i k} t_{i} s_{i k} d t_{k}$,

das Nutzenniveauänderungen aufgrund kleiner Steuerbetragsvariationen aufzeigt ${ }^{1}$ ). Formal ausgedruickt lautet das Problem: Gibt es Anderungen der $t_{k}$, so daß dW >0 gilt?

Es kann gezeigt werden, daß Anderungen der Steuerbeträge pro Mengeneinheit genau dann zu einer Wohlfahrtssteigerung führen, wenn die rechte Seite von (II.49) größer als null wird ${ }^{2}$.

\section{II.6. Die Interpretation der Steuerreformergebnisse}

Wie im vorigen Abschnitt wird nunmehr eine proportionale Senkung aller Steuersätze unterstellt $\left(d t_{j}=-b t_{j}\right)$, weshalb (II.49) die Form

(II.50) $\quad-\underset{i k}{b \sum_{i k}} t_{i k} t_{k}>0$

annimmt.

Da die Matrix der Substitutionseffekte negativ definit ist, wird (II.50) positiv, und eine gleiche proportionale Senkung der Steuersätze pro Mengeneinheit erhöht das Nutzenniveau des Konsumenten $^{3)}$. Demnach gilt:

OT-Rege 16 :

Eine schrittweise proportionale Senkung aller Steuersätze ist

1) Vg1. auch Dixit (1975), S. 107 oder Wiegard (1978), S. 105

2) $\mathrm{Vgl}$. dazu eine ausführliche Ableitung des Wohlfahrtskriteriums mit Hilfe der indirekten Nutzenfunktion, Mann (1983). S. $28 \mathrm{ff}$. Zum Bewe is siehe z.B. Wiegard (1978), S. $188 \mathrm{ff}$.

3) Dieses Ergebnis leitet z.B. Green (1975), S. 363 ab. Dixit (1975), S. 122 ff., zeigt, daß das Ergebnis auch im Falle abnehmender Skalenerträge gilt. 
effizienzsteigernd, wenn der daraus resultierende Steuerausfall durch eine entsprechende Erhöhung der Pauschalsteuern kompensiert wird. Dies bedeutet auch, daB eventuell bestehende Pauschaleinkommen der Haushalte zugunsten der vorgeschlagenen Steueränderung abgebaut werden müßten, und, wenn möglich, auch Pauschalsteuern eingeführt werden sollten ${ }^{i)}$.

Allerdings führt nicht jede Substitution von Gütersteuern durch Pauschalsteuern zu Wohlfahrtsverbesserungen. Gleichung (II.50) kann nämlich auch folgendermaßen geschrieben werden:

$$
\begin{aligned}
& \left(t_{1} s_{11} d t_{1}+\ldots+t_{n} s_{n n} d t_{n}\right)+ \\
& \left(t_{1} s_{12} d t_{2}+\ldots+t_{1} s_{1 n} d t_{n}\right)+ \\
& \text { - } \\
& \text { - } \\
& \text { - } \\
& \text { - } \\
& \left(t_{n} s_{n 2} d t_{2}+\ldots+t_{n} s_{n(n-1)} d t_{n-1}\right)>0 .
\end{aligned}
$$

Nimmt man eine Steuersenkung $\left(d t_{i}<0\right)$ an, dann wird für $\left(\partial x_{i} / \partial p_{i}=s_{i}<0\right)$ die erste Zeile in (II.51) gröBer als null.

Bestehen zwischen einem Teil der besteuerten Güter Substitutionsbeziehungen der Form $\left(\partial x_{i} / \partial p_{k}=s_{i k}>0\right)$, während $z$ wischen den übrigen die Kreuzpreiselastizitäten vernachlässigt werden können, so wird der Restausdruck von (II.51) negativ. Je nach Stärke der Preiseffekte und Steuersatzänderungen kann (II.51) kleiner als null werden, d.h. eine Substitution von indirekten Steuern durch Pauschalsteuern kann Wohlfahrtsverluste nach sich ziehen ${ }^{2}$. Dieses Ergebnis mag verwundern, nachdem in OT-Regel 1 abgeleitet wurde, daB die Finanzierung des Steueraufkommens durch Pauschal-

1) Vgl. Z.B. Munk (1978), S. 12, siehe auch die OT-Regel in Anhang A.

2) Vgl. dazu Wiegard (1980), S. 8 oder auch Atkinson/Stiglitz (1980), S. 383 
steuern erfolgen sollte. Die SchluBfolgerung, daB aus jeder Steuerstrukturänderung zugunsten von Pauschalsteuern Wohlfahrtsgewinne resultieren, ist falsch. Sie ist nur gewährleistet, wenn die Güter- und Faktorsteuern nicht beliebig geändert werden, sondern derart, daß sie bei jedem Pauschalaufkommensniveau eine optimale Second-Best-struktur aufweisen.

Gleichung ( II.51) belegt, daB eine effizientere Form eines suboptimalen Steuersystems nur unter Berücksichtigung und Kenntnis der Substitutions- und Komplementaritätsbeziehungen zwischen den Gütern (Faktoren) gelingen kann ${ }^{1)}$. Dies bedeutet, daB man für kleine ebenso wie für große Steuerreformen in der Regel dieselben Plandaten benötigt ${ }^{2}$. Demnach gilt:

Steverreformregel 2 :

Steuerreformen im Sinne der OT können nur dann erfolgreich sein, wenn alle relevanten Verhaltensparameter der Besteuerten bekannt sind. Dies impliziert die Kenntnis von Preis-, Kreuzpreis- und Einkommenselastizitäten.

Diese Steuerreformregel ist deshalb von größerer finanzpolitischer Bedeutung, weil die Tatsache berücksichtigt wurde, daB Steuerstrukturänderungen nur inkrementalistisch vonstatten gehen und nur ausgehend von dem aktuellen, historisch gewachsenen Zustand des Steuersystems möglich sind. Daraus resultiert schließlich:

1) Vgl. Wiegard (1980), S. 11

2) Im Falle von Pauschalsteuern benötigen kleine Reformen, also eine sukzessive Erhöhung der Pauschalsteuern, mehr Informationen als eine große Steuerreform, die das gesamte Steueraufkommen durch die Pauschalsteuer erbringt. Die Bedeutung der Substitutionseffekte wird auch klar, wenn man von einer einheitlichen Besteuerung ausgehend sukzessive eine optimale, differenzierte Steuerstruktur erreichen will. Mit dem Wohlfahrtskriterium (II.49) läßt sich beispielsweise auch nachweisen, daß eine sukzessive Erhöhung der Steuern auf jene Güter, die zu dem unbesteuerten Gut komplementär sind, das Wohlfahrtsniveau steigern, wenn gleichzeitig die Substitutionsguiter niedriger besteuert werden. Vgl. Z.B. Wiegard (1980), S. 12 
Steverreformregel 3 :

Vor jeder noch so kleinen Steuerstrukturänderung müssen die Steuerinstrumente, die Steuerbemessungsgrundlage und das Nachfrageverhalten der Individuen bekannt sein (Steuerreformregel 1 und 2 ). Im Gegensatz zu den OT-Regeln 1 - 5 ist nun nicht mehr allein eine als optimal identifizierte Steuerstruktur von Bedeutung, sondern vor allem das aktuelle Steuersystem. Dabei ist zu beachten, daB nicht jede Umstrukturierung in Richtung eines optimalen Steuersystems tatsächlich zu einer Wohlfahrtsverbesserung führt.

Neben den qualitativen Aussagen der einzelnen Steuerregeln ist aber auch ihre Quantifizierung von größter Bedeutung, weil nur so das AusmaB der Steuersatzunterschiede zwischen den einzelnen Gütern bzw. Gütergruppen bestimmt werden kann. Bevor im folgenden die Problematik der empirischen Ermittlung optimaler Steuerstrukturen exemplarisch aufgezeigt wird, scheinen einige Bemerkungen zur Relevanz des allgemeinen Gleichgewichtsmodells angebracht.

\section{II.7. Die Relevanz des allgemeinen Gleichgewichtsmodells}

Die Ableitung optimaler Steuerreformen im allgemeinen Gleichgewichtsmodell hat den Vorteil, daB alle Güter und Faktoren sowie die wesentlichen Aktivitätszentren einer Volkswirtschaft simultan beruicksichtigt werden können. Es ist daher möglich, alle Märkte und ihre gegenseitigen Einflußfaktoren bei der Optimierung zu berücksichtigen, wenngleich dies häufig zu sehr komplizierten OTRegeln führt.

Gleichzeitig weist das Modell jedoch erhebliche Mängel in bezug auf die Problemstellung auf. Erstens bezieht sich das komparativ statische Modell auf eine geschlossene Volkswirtschaft und berücksichtigt Marktunvollkommenheiten und die Produktionsseite der Volkswirtschaft nur sehr unvollkommen. Obgleich die Unzulänglichkeiten prinzipiell behebbar sind, muß auf den ungeheueren Informationsbedarf hingewiesen werden, wenn tatsächlich alle Marktunvollkommenheiten und die produktionstechnische Seite modelijert 
werden müßten ${ }^{1)}$. Der oben erwähnte Vorteil eines umfassenden Modells hat daher den Nachteil hoher Informationskosten ${ }^{2}$ ). Eine weitere wesentliche Schwäche des Modells rührt aus der Vernachlässigung der Transaktionskosten. Wesentliche Kosten, die bei jeder Steuerreformdiskussion im Mittelpunkt stehen ${ }^{3)}$, wie $z . B$. Verwaltungskosten ${ }^{4}$ ). Steuererhebungskosten, Kontrollkosten oder Fragen der Steuerkriminalität ${ }^{5}$ ) werden ausgeklammert. Darüber hinaus müßten die nicht unerheblichen Informations- und Anpassungskosten der Zensiten berücksichtigt werden. Dennoch ist der Wert der theoretischen Erkenntnisse durch das vorliegende Modell offenkundig. Wird die OT als Grammatik von Steuersystemzusammenhängen verstanden, kann sie auch wertvolle Dienste in der praktischen Finanzpolitik leisten ${ }^{6)}$. Dagegen wird der Wunsch nach einer konkreten Ausgestaltung, d.h. einer Ouantifizierung der OT-Regeln, Utopie 7) bleiben.

1) Vgl. dazu Sohmen (1976), S. $436 \mathrm{f}$.

2) Erst in jüngster Zeit werden Auswirkungen von Unsicherheit auf die optimalen Steuerentscheidungen berücksichtigt. Dabei muß zwischen unsicheren Entscheidungen der Regierung über die Ausgestaltung eines optimalen Steuersystems (vgl. Z.B. Kap. VII dieser Arbeit) und der Unsicherheit der Steuerzahler über künftige Entwicklungen unterschieden werden. Vgl. im Zusammenhang mit letzterer Problemstellung Z.B. die Arbeiten von Eaton/ Rosen (1980 a und b) oder Varian (1981). Diamond/Helms/Mirrlees (1980), zeigen mit ihren Simulationen, daß die Berücksichtiqung von Unsicherheit zu äußerst sensiblen und komplexen Ergebnisstrukturen führt.

3) Vgl. Z.B. Ben-Porath/Bruno (1977), S. 289

4) Vgl. Haller (1973/74), S. 31. Nur wenige OT-Arbeiten beschäftigen sich explizit mit der Verwaltungsproblematik. Es ist daher die Ausnahme, wenn $z . B$. bei Stern (1982) Informationsmängel in der Steuerverwaltung analysiert werden. Externe Effekte, Besteuerung und administrative Kosten werden bei Pol insky/Shavell (1982) analysiert. Institutionelle Fragen, wie z.B. Steuerharmonisierung in der EG waren für die Steuerreformkommission (1971), S. $34 \mathrm{f}$. ein wesentlicher Gesichtspunkt.

5) Fragen der Steuervermeidung und Steuerhinterziehung werden i.d.R. im Rahmen der Spieltheorie und Risikoanalyse berücksichtigt. Vgl. z.B. Allingham, Sandmo (1972), Friedland, Maital, Rutenberg (1978), Baldry (1979), Christiansen (1980) oder Koskela (1983)

6) Vgl. Hahn (1973), S. 106

7) Vgl. zu einer kritischen Einschätzung Broome (1975), S. 649 ff. 


\section{II.8. Zusammenfassung}

Am vorliegenden Modell sollen Vor- und Nachteile der optimal taxation aufgezeigt werden. Ferner soll überprüft werden, ob sie als finanzpolitische Basis für Steuerreformen dienen kann. Es wurde demonstriert, daB optimale Steuerregeln im wesentlichen von der Art des Steuerinstrumentariums, der Steuerbemessungsgrundlage und den Präferenzen der Individuen abhängen. Variationen dieses Datenkranzes führen daher im allgemeinen zu Anderungen der optimalen Steuerregeln. Da auf politischer Ebene permanente Variationen des Datenkranzes vorgenommen werden, müßten ununterbrochen neue optimale Steuersysteme ermittelt und angestrebt werden. Wollte man die optimalen Endzustände mit kleinen Reformen erreichen, hieBe das permanente Richtungsänderungen kleiner Steuerreformen. Bei näherer Betrachtung der theoretischen, verwaltungs- und informationstechnischen Schwierigkeiten und Kosten für die Ermittlung eines einzigen optimalen Steuersystems oder einer einzigen optimalen Steuerreform wird die Utopie des Unterfangens schnell deutlich. Daraus folgt unmittelbar die wichtige Erkenntnis, daß vor jeder Steuerreform langfristig konstante Rahmenbedingungen vorgegeben werden müssen, die auf politischer Ebene nicht beliebig variiert werden können. Aber selbst wenn diese für eine langfristige Reformstrategie notwendigen Voraussetzungen erfuilt sind, bleiben vielfältige Informationsprobleme bestehen, so daß die Quantifizierung optimaler Steuerregeln kaum eine realistische Perspektive darstellt. 


\section{DIE INFORMATIONSPROBLEMATIK OPTIMALER STEUERREFORMEN}

Die im vorangegangenen Kapitel abgeleiteten OT-Regeln machen den hohen Informationsbedarf der optimal taxation (OT) deutlich. Aus finanzpolitischer sicht sind vor allem die Informationsrisiken einer quantitativen Ausgestaltung optimaler Steuersysteme von Interesse. Die Informationserfordernisse des vorliegenden Modells bestehen im wesentichen aus:

1) der Entwicklung eines adäquaten Modellrahmens

2) der Spezifikation eines geeigneten Angebots - und Nachfragesystems und

3) der ökonometrischen Schätzung der Elastizitätswerte.

Punkt 1) zielt auf die grundsätzliche Frage, ob innerhalb des Modellrahmens tatsächlich alle für eine Steuerreform relevanten Daten sowie politische und ökonomische Restriktionen (vgl. Abb. 1, S. 12) berücksichtigt werden. Damit ist auch die finanzpolitische Bedeutung der OT-Modelle angesprochen, die im AnschluB an die Informationsproblematik diskutiert wird.

\section{II.1. Die empirische Sensibilität optimaler Steuerregeln}

III.1.1. Zur Spezifikation von Nachfragesystemen

Die bisher abgeleiteten Steuerregeln zeigen die Steuersätze in Abhängigkeit von den Preis-, Kreuzpreis-oder Einkommenselastizitäten der Angebots- und Nachfragefunktionen. Die Elastizitäten wiederum werden durch die Präferenzen der Individuen determiniert, die in der okonomie üblicherweise durch Nutzenfunktionen formalisiert werden. Um Nutzenfunktionen für ökonometrische Schätzungen von Nachfragesystemen brauchbar zu machen, müssen die verwendeten Funktionen ganz bestimmten Anforderungen genügen. Die unter der Nebenbedingung einer Budgetrestriktion abgeleiteten Nachfragefunktionen, die für die ökonometrischen Schätzungen die Modellgrundlage bilden, spiegeln dann natürlich diese Funktionseigenschaften 
wider ${ }^{1}$. Im folgenden soll gezeigt werden, daß die Wahl bestimmter Nutzenfunktionstypen die ökonometrischen Schätzungen und damit vor allem die für die optimalen Steuersätze wichtigen Substitutionsbeziehungen entscheidend beeinflußt ${ }^{2}$ ). Zu diesem Zweck werden beispielhaft drei Funktionstypen vorgestellt, die in der okonometrie sehr häufig für Schätzungen von Nachfragesystemen verwendet werden. Dies liegt wohl auch daran, daß die Zahl der mathematisch akzeptablen Nutzenfunktionen eng begrenzt ist ${ }^{3)}$. Die Darstellung der Schwierigkeiten einer geeigneten Spezifikation von Nachfragesystemen soll jedoch nicht zu der Auffassung führen, daß die Ermittlung von Elastizitätswerten sinnlos oder gar unmöglich sei. Vielmehr soll gezeigt werden, daß es großer Anstrengungen bedarf, um verläßliche Schätzungen durchführen zu können.

\section{II.1.1.1. Additive Nutzenfunktionen}

Der Funktionstyp mit den restriktivsten Eigenschaften wird durch additive Nutzenfunktionen repräsentiert. Additivität von Nutzenfunktionen bedeutet, daß der Grenznutzen eines jeden Gutes unabhängig von den konsumierten Mengen aller anderen Güter ist ${ }^{4}$. Die Klasse von Nutzenfunktionen, die additive Präferenzordnungen repräsentieren, läßt sich dann wie folgt schreiben:

$$
\text { (III.1) u }=\sum_{i} f_{i}\left(x_{i}\right)
$$

mit der Bedingung

1) "This is important since the validity of any set of estimated elasticities must depend upon the appropriateness of the postulates adopted in the models used to derive these estimates." Brown/Deaton (1972), S. 1157

2) "It should then be clear that the choice of demand model itself has important implications; strong a priori notations are built into the analysis by the choice of model and these will interact with the data to yield results which to some extent will be affected by the models chosen." Brown/Deaton (1972), S. 1152

3) Vgl. Phlips (1974), S. 93

4) Vg1. Brown/Deaton (1972), S. 1166 
(III.2) $\frac{\partial^{2} u}{\partial x_{i} \partial x_{j}}=0$.

Wählt man nun beispielsweise die Nutzenfunktion

(III.3) $\quad u=\sum_{i} \log x_{i}$

und maximiert sie unter der Nebenbedingung

(III.4) $\quad y=\sum_{i} p_{i} x_{i}$,

so erhält man Nachfragefunktionen, die lediglich das exogene Einkommen und den eigenen Preis des jeweiligen Gutes als Argument in der Funktion enthalten:

(III.5) $\quad x_{i}=x_{i}\left(p_{i}, y\right)$.

Natürlich verschwinden unter diesen Bedingungen die Kreuzpreiseffekte, so daß im Falle der Gültigkeit dieser Nachfragefunktionen die inverse Elastizitätsregel zur Anwendung gelangen würde ${ }^{1)}$. Die Funktionstypen, die gleichzeitig direkte oder indirekte Additivität aufweisen, haben gleiche Einkommenselastizitäten und, wegen der fehlenden Kreuzpreiselastizitäten, gleiche eigene Preiselastizitäten ${ }^{2}$. Nutzenfunktionen dieser Art sind eine Subklasse der homothetischen Nutzenfunktionen.

\section{1.1.2. Homothetische Nutzenfunktionen}

Die Klasse der homothetischen Nutzenfunktionen ist in bezug auf das beobachtbare Konsumentenverhalten ebenfalls sehr restriktiv, so daß sie nicht als Grundlage zur Schätzung der gesuchten Elastizitätswerte herangezogen werden kann.

1) Vgl. Atkinson/Stiglitz (1972)

2) Vgl. Phlips (1974), S. 65 f. 
Eine Funktion wird homothetisch genannt, wenn sie in der Form $u=F\left[f\left(x_{1}, \ldots x_{n}\right)\right]$ geschrieben werden kann, wobei $F$ eine positive, endliche, stetige und streng monoton zunehmende Funktion einer Variablen mit $F(0)=0$ ist und $f$ eine homogene Funktion von $n$ Variablen ${ }^{1)}$ bezeichnet. Zu den homothetischen Nutzenfunktionen gehört beispielsweise auch eine Nutzenfunktion vom Cobb-Douglas-Typ:

(III.6) $\quad u=\sum_{i} B_{i} \log x_{i}$, mit $\sum_{i} B_{i}=1$.

Da homothetische Nutzenfunktionen nur bis auf eine monoton zunehmende Transformation bestimmt sind, genügen sie gleichzeitig den Bedingungen homogener Funktionen, die einheitliche Einkommenselastizitäten besitzen. Dies bedeutet, daß ein konstanter Anteil der Gesamtausgaben für jedes in der Nutzenfunktion enthaltene Gut ausgegeben wird. In der Indifferenzkurvengeometrie bedeutet dies, daß die Engel-Kurven Geraden sind, die durch den Ursprung gehen, und daB die Nachfrage nach den Gütern entlang dieser Engel-Kurve ausgedehnt bzw. eingeschränkt wird. Die aus diesen Nutzenfunktionen ableitbaren Nachfragefunktionen implizieren Ausgabenproportionalität; ein solches Nachfrageverhalten widerspricht jedoch dem beobachtbaren Konsumentenverhalten ${ }^{2}$ ).

\section{III.1.1.3. Stone-Geary-Funktionen}

Der dritte Funktionstyp, der diese Nachteile umgehen soll, besteht aus separablen Nutzenfunktionen ${ }^{3)}$, die ihrerseits wiederum in streng separable und schwach separable Nutzenfunktionen untergliedert werden. Es sei an dieser Stelle nur auf die am wenigsten restriktiven, schwach separablen Nutzenfunktionen eingegangen, da

1) Vgl. z.B. Phlips (1974), S. 86 oder Lau (1970); zu den Implikationen für optimale Steuerstrukturen siehe z.B. Wiegard (1976), S. $208 \mathrm{f}$.

2) Vgl. Phlips (1974), S. 88, Brown/Deaton (1972), S. 1167

3) Zur Verwendung von separablen Nutzenfunktionen in der Nachfrageanalyse siehe Z.B. Goldman/Uzawa (1964), S. 387 - 398. Dort werden die Eigenschaften von drei Separabilitätskonzepten diskutiert, sowie die Bedingungen z:r Gruppierung von Gütern. 
die streng separablen Nutzenfunktionen unter bestimmten Bedingungen mit den additiven Nutzenfunktionen im ökonomischen Sinne identisch sind. Separable Nutzenfunktionen zeichnen sich dadurch aus, daß fütergruppenaggregate in den Nutzenfunktionen erscheinen. Separabilität impliziert dann, daß der Grenznutzen von Gütern, die zu einer Gruppe gehören, unabhängig von den konsumierten Mengen aller anderen Güter außerhalb dieser Gruppe ist. Die Funktionen haben weiterhin die Eigenschaft, daB innerhalb der Gruppen und zwischen den fruppen als ganzen beliebig substituiert werden kann, da $B$ aber Restriktionen bestimmter Güter auf Veränderungen außerhalb ihrer Grupde mit den Gruppeneigenschaften konform gehen müssen. Die Klasse der schwach separablen Nutzenfunktionen läßt. sich dann wie folgt schreiben:

(III.7)

$$
u=f\left[u_{1}\left(x_{1}\right), \ldots, u_{n}\left(x_{n}\right)\right] \text { mit } i=1, \ldots, n \text {, }
$$

wobei $x_{i}$ der Gütermengenvektor der $i$-ten Gruppe ist.

Eine für ökonometrische Schätzungen sehr häufig verwendete Nutzenfunktion der so definierten Klasse ist die Stone-Geary-Funktion ${ }^{1}$ :

$$
u=\sum_{i} \beta_{i} \log \left(x_{i}-\gamma_{i}\right) \text {, mit } \sum_{i} B_{i}=1
$$

Maximiert man die Stone-Geary-Funktion unter der Nebenbedingung der Budgetrestriktion, so lassen sich die Nachfragefunktionen

$$
x_{i}=\gamma_{i}+\frac{\beta_{i}}{D_{i}}\left(y-\sum_{k} p_{k} \gamma_{k}\right)
$$

bzw.

$$
p_{i} x_{i}=\gamma_{i} p_{i}+\beta_{i}\left(y-\sum_{k} p_{k} \gamma_{k}\right)
$$

ableiten, die auch als ineares Ausgabensystem bekannt sind ${ }^{2}$ ). Die

1) Dieser Funktionstyp ist vor allem mit den Arbeiten von Stone (1954), Geary $(1950 / 51)$ und Samuelson (1947/48) verbunden.

2) Vgl. Brown/Deaton (1972), S. 1196 f., Phlips (1974), S. 125 
Nachfragefunktionen können dann gemäß Samuelson ${ }^{1)}$ wie folgt inter pretiert werden: Die Ausgaben für Gut $i$ sind durch $p_{j} x_{i}$ gegeben. Sie werden einerseits in die minimalen Ausgaben $p_{i} \gamma_{j}$ zerlegt, die zur Erhaltung des Existenzminimums notwendig sind. Die Summe aller Minimalausgaben $\sum_{i} p_{i} \gamma_{i}$ kann als Subsistenzeinkommen aufgefaßt werden. In ökonometrischen Schätzungen sind dann die Mindestgütermengen $\gamma_{i}$ und die Konstanten $\beta_{i} z u$ ermitteln.

Die Stone-Geary-Funktionen zeigen jedoch eine (merkwürdige) Elastizitätseigenschaft, die ein Steuersystem von vornherein mitbestimmt. Da die dem 1 inearen Ausgabensystem zugrundeliegende StoneGeary-Funktion schwach separabel zwischen Arbeit (dem Numérairegut) und den Konsumgütern ist, werden sich mit Hilfe der RamseyRegel arundsätzlich uneinheitliche Verbrauchsteuersätze ergeben ${ }^{2}$. Einheitliche Steuersätze wären daher nur zufällig optimal ${ }^{3)}$. Es läßt sich nämlich zeigen, daß die Stone-Geary-Funktionen lediglich Substitutionsqüter im Hicks-Allen-Sinn ${ }^{4}$ definieren. Nach Hicks werden Substitutionsgiter durch einen positiven Kreuzpreiseffekt definiert; und in der Tat zeigen alle Kreuzpreiseffekte der Stone-Geary-Funktionen einen positiven Wert ${ }^{5}$ ). Die Hicks'sche Definition bringt zum Ausdruck, daß ein konsument, derdurch Ein-

1) Val. Samuelson $(1947 / 48)$, S. $88 \mathrm{f}$.

2) $\mathrm{Vgl}$. Z.B. Deaton (1981), S. 1251. Deaton weist auch im Mehr-Personen-Model1 nach, daß: "under weak separability no discrimination is required so that in the presence of an optimal non-linear income tax, commodity taxation is necessary." Deaton (1981), S. 1252

3) Durch die Kombination der Eigenschaften homothetischer und schwach separabler Nutzenfunktionen kann die Bedeutung der in ökonometrischen Schätzungen unterstellten Nutzenfunktionen weiter verdeutlicht werden. Setzt man nämlich homothetische Nutzenfunktionen in den Konsumgütern voraus, wie die Annahme, daß die Nutzenfunktionen schwach separabel zwischen Arbeit und Freizeit seien, dann sind einheitliche Steuersätze optimal. Zum Bewe is siehe Sandmo (1974), S. 705 oder in modifizierter Form Wiegard (1976), S. 208 f.

4) Zur ausführlichen Diskussion der Hicks-Definition siehe Z.B. Brown/Deaton (1972), S. 1196 oder Phlips (1974), S. $77 \mathrm{ff}$. und zur Definition siehe Hicks (1939), Kap. III, S. 42 - 52

5) "It may seem odd that since complementary goods are defined in terms of the theory, a system [...] should be unable to take account of this phenomenon." Brown/Deaton (1972), S. 1196. Zu einem ausführlichen Bewe is dieser Behauptung siehe Phlips (1974), S. $60-62$ 
kommenskompensationen immer auf der gleichen Indifferenzkurve gehalten wird, bei einem Preisanstieg dazu veranlaßt wird, Gut 2 gegen Gut 1 zu substituieren. Im Falle der Substitute bedeutet dies, daß ein Konsument, der für den Preisanstieg bei Butter einkommensmäßig so kompensiert wird, daß er stets auf der gleichen Indifferenzkurve bleibt, vom Gut Margarine mehr kauft. Der Ausschluß von Komplementärgütern wie z.B. Kaffee und Zucker oder Freizeit- und Campingausrüstung, deren Elastizitätswerte in der Theorie der optimalen Besteuerung eine große Bedeutung haben, ist sicher ein wesentlicher Nachteil für die oben abgeleiteten Nachfragesysteme ${ }^{1)}$. Ein Beleg für die Sensitivität der Elastizitätswerte in bezug auf die Spezifikation von Nachfragesystemen findet sich bei Abbott/Ashenfelter ${ }^{2}$ ). Ihre Schätzungen zeigen, daB die kompensierten eigenen Preiselastizitäten und Kreuzlohnelastizitäten von Modell zu Modell zum Teil erheblich variieren. Harris/ Mackinnon ${ }^{3)}$ bestätigen diese Resultate. Sie untersuchen mittels einer Stone-Geary-Nutzenfunktion die Effekte der Änderungen von Substitutionselastizitäten auf die Steuersätze, wobei ein gegebenes Steueraufkommen erzielt werden muß. Geschätzt wird ein lineares Ausgabensystem mit fixem Arbeitseinkommen und konstanten Produzentenpreisen. Ihre Ergebnisse zeigen, daß die Steuersatzänderungen mit zunehmenden absoluten Elastizitätswerten zunehmen. Außerdem hängt die Struktur der Steuersätze entscheidend von der Höhe des gewünschten Steueraufkommens ab.

\section{II.1.2. Weitere Probleme der empirischen Analyse}

Neben der Kritik an den äußerst restriktiven Modellspezifikationen seien noch die Schwierigkeiten der Identifikation und Aggregation

1) Zu weiteren Schätzproblemen der I inearen Nachfragesysteme siehe Brown/ Deaton (1974), S. 1197

2) Vgl. Abbott/Ashenfelter (1976), insbesondere S. 399

3) "They [the sample estimation (vom Autor hinzugefuigt)] suggest that optimal tax rates are exceedingly sensitive to the specification of the model used to drive them." Harris/Mackinnon (1979), S. 197. "Thus optimal tax theory should be used with extreme caution until a great deal more is known about the specification of realistic systems of consumer demand and labour supply functions." Harris/Mackinnon (1979), S. 212 
von Nachfragefunktionen erwähnt. Das Identifikationsproblem tritt auf, weil nur die am Markt erzielten Preise beobachtet werden können, die lediglich den Schnittpunkt der Angebots- und Nachfragekurve zu einem bestimmten Zeitpunkt anzeigen. Das Herausfiltern der relevanten Nachfragekurven ist daher nur unter bestimmten Voraussetzungen oder Hypothesen wie z.B. der Konstanz der Produzentenpreise möglich ${ }^{1}$ ).

Das Aggregationsproblem besteht darin, daB die individuellen Nachfragefunktionen nur unter sehr restriktiven Bedingungen zu einer Gesamtnachfragefunktion aggregiert werden können. Umgekehrt bedeutet dies, daß aus den aggregierten Daten vieler Haushalte keinesfalls auf ein mit der Nachfragetheorie kompatibles, individuelles Konsumentenverhalten rückgeschlossen werden kann. Damit die am Markt ermittelten Aggregate so interpretiert werden durfen, als seien sie die Ergebnisse der Entscheidungen eines einzelnen, nutzenmaximierenden Konsumenten, müßten die Engel-Kurven aller Individuen parallele Geraden sein ${ }^{2}$, d.h. alle Güter müBten die gleichen Einkommenselastizitäten aufweisen. Diese Voraussetzungen widersprechen dem beobachtbaren Konsumentenverhalten.

Da die angedeuteten Probleme beim gegenwärtigen Stand der Theorie nicht oder nur sehr unvollkommen gelöst werden können, pflegt man sie in der Praxis zu übergehen. Beispielsweise werden die aggregierten Daten auf ein "repräsentatives" Wirtschaftssubjekt bezogen, wenngleich sich dessen Verhalten nicht aus dem konkreten Verhalten der individuellen Nachfrager ableiten läßt ${ }^{3}$. In Konsequenz kann eine optimale Steuerreform nur für diesen Repräsentanten, nicht aber für den individuellen Konsumenten abgeleitet werden. Es erscheint zumindest fraglich, ob die Inkonsistenz zwischen Makrodaten und Mikrotheorie eine tragbare Basis für die optimale Steuertheorie bietet ${ }^{4}$ ).

1) Vgl. Deaton/Brown (1972), S. 1188

2) Vgl. Phlips (1974), S. 98, Deaton/Muellbauer (1980), S. 158

3) Vgl. Phlips (1974), S. 100

4) Vgl. Deaton/Muellbauer (1980), S. 148 
Die skizzierten Problempunkte sollen nicht zur Resignation vor der empirischen Ermittlung von Elastizitätswerten führen. Im Gegenteil, die empirische Forschung ist trotz ihrer Mängel unverzichtbar, um die praktische Relevanz theoretischer Oberlegungen wenigstens in der Tendenz testen zu können. Ebenso wertvoll erscheinen die Sensitivitätsanalysen im Rahmen der OT-Modelle. Durch siewerden Unsicherheitsbereiche einer quantitativen Ausqestaltung der OT-Regeln besonders deutiich.

Trotz hilfreicher Einsichten auf der quantitativen Seite optimaler Steuersysteme müssen die Ergebnisse der ökonometrischen Schätzungen sehr skeptisch beurteilt und noch vorsichtiger interpretiert werden ${ }^{1)}$. Denn abgesehen von der Frage nach der ökonomischen Bedeutung der beispielsweise aus Zeitreihen stammenden Daten (Identifikationsproblem, Aggregationsproblem), ist die Spezifikation der Nachfragesysteme, die ihrerseits nur sehr restriktive Verhaltensweisen der Konsumenten implizieren, von ausschlaggebender Bedeutung für die abzuleitenden Elastizitätswerte. Die Elastizitätswerte, aber auch andere GröBen wie z.B. die Höhe des Budgets und die Zahl der im Modell berücksichtigten Güter bzw. Gütergruppen, determinieren die Struktur der Steuersätze. Der starke Einfluß der Modellspezifikation auf die ökonometrischen Schätzungen nährt den Verdacht, daß die Elastizitätswerte nicht aus dem aktuellen Nachfrageverhalten der Konsumenten ermittelt werden, sondern durch willkürliche, ungetestete Hypothesen, die sich in der Modellspezifikation niederschlagen ${ }^{2}$. Aus finanzpolitischer sicht ergeben sich daher folgende Forderungen:

1) Vgl. Z.B. die Schätzungen von Deaton (1975), (1978) oder von Parks (1969), die die Elastizitätswerte mit Hilfe verschiedener Nachfragesysteme schätzen. Der Vergleich zeiqt, daß: "The estimates of income and own compensated price elasticities showed statistically insignificant differences for some commodities, but for others the differences were large and significant." Parks (1969), S. 649

2) Vgl. Deaton (1981), S. 1245: "In consequence, it is likely that empirically calculated tax rates, based on econometric estimates of parameters, will be determined in structure, not by the measurement actually made, but by arbitrary, untested (and even unconscious) hypotheses chosen by econometricians for practical convenience." 
(1) Es sollten Methoden entwickelt werden, die eine exakte Messung der relevanten Parameter zulassen, statt durch implizite Funktionsannahmen die Steuerstruktur von vornherein $z u$ determinieren ${ }^{1}$ ).

(2) Geeignete Modellspezifikationen müsen daher sehr viel realitätsnähere Annahmen bei der Formulierung von Nutzenfunktionen berücksichtigen.

(3) Ferner muß es möglich sein, die Elastizitäten einer ausreichend großen Zahl von Gütern bzw. Gütergruppen zu bestimmen. Eine beliebig starke Desaggregation der Güter ist mit den bisherigen Modellen nicht möglich. Die Problematik wird deutlich, wenn man bedenkt, daß bei 20 fiütergruppen schon 400 Preiselastizitäten ermittelt werden müssen.

(4) Für finanzpolitische Entscheidungen ist es weiterhin wichtig, daß die Elastizitätswerte über längere Zeit konstant sind und klare Trends aufweisen. Diese Forderungen sind bisher nicht erfüllt, und es wird auch in Zukunft in vielen Bereichen schwierig sein, verläßliche Elastizitätswerte zu ermitteln, weil technischer Fortschritt und Präferenzänderungen die Komplementarität bzw. Substitutionsbeziehungen zwischen einzelnen Gütern und Gütergruppen ändern.

Als Fazit bleibt festzuhalten, daB in der Okonometrie kein Nachfragemodell Anwendung findet, das das aktuelle Konsumentenverhalten in akzeptabler Weise widerspiegelt. Unterstellt man, daß die ökonometrischen Methoden wesentlich verbessert werden können, wird die angeführte Kritik (hoffentlich) nur temporärer Natur sein ${ }^{2}$ ).

1) Vgl. auch Deaton (1981), S. 1259

2) In neueren ökonometrischen Arbeiten versucht man daher, einen anderen Weg zu gehen, indem man nicht mehr die gesamte Nachfragefunktion als verfügbar voraussetzt, sondern mit einer begrenzten Anzahl von Beobachtungen über das Konsumentenverhalten auszukommen versucht. Der Verzicht auf eine vorgegebene Funktionsform muß jedoch damit erkauft werden, daß die nicht-parametrischen Tests für große Datenmengen nicht gelöst werden können.Vgl. dazu Varian (1983), S. 99 f. und die dort angegebene Literatur. 
Auf absehbare Zeit scheint es jedoch wenig sinnvoll zu sein, die wenigen, mit erheblichen Mängeln behafteten, empirischen Untersuchungen als Ausdruck der Realität anzusehen. Vor der quantitativen Ausgestaltung der OT-Regeln und ihrer finanzpolitischen Akzeptanz sind noch viele theoretische und praktische Probleme der Informationsgewinnung zu lösen.

\section{II.2. Zur finanzpolitischen Relevanz von Ein-Konsumenten-Modellen}

Die OT-Regeln im Ein-Konsumenten-Modell beruhen auf der Fiktion eines repräsentativen Individuums innerhalb einer Gesellschaft identischer Wirtschaftssubjekte ${ }^{1)}$. Die Effizienzregeln des Ein-Konsumenten-Modells sind daher uneingeschränkt nur für eine spezifische Präferenzstruktur gültig. In der Realität unterscheiden sich die Präferenzen der Individuen in einer Gesellschaft z.T. erheblich, so daB die Anwendung einer einzigen OT-Regel auf alle Individuen nicht zwingend zu einer Pareto-Verbesserung führen muB. Für abweichende Präferenzmuster wird die allgemein gültige Regel definitionsgemäß suboptimal sein. Je stärker das Konsumentenverhalten innerhalb einer Gesellschaft differiert, um so geringer wird der Spielraum für eine einheitliche Effizienzregel ${ }^{2}$ ).

Die Behauptung leuchtet sofort ein, wenn man in einer Zwei-Konsumenten-Gesellschaft davon ausgeht, daß ein Individuum $A$ ein homothetisches Indifferenzkurvensystem besitzt, das zwischen Arbeit und Konsumguitern schwach separabel ist, während das Individuum $B$ eine Stone-Geary-Nutzenfunktion aufweist. Im ersten Fall wäre ein einheitliches Verbrauchsteuersystem optimal, während im zweiten Fall einheitliche Steuersätze suboptimal sind. Geht man von einem Verbrauchsteuersystem mit einheitlichen Steuersätzen aus, würde die Differenzierung der Verbrauchsteuer gemäß dem Präferenzmuster des Individuums $B$ das Individuum $A$ benachteiligen. UnterläBt man

1) Ein Modell mit einer Zwei-Klassenökonomie wurde von Mirrlees (1975) entwickelt.

2) $\mathrm{Vgl.zu}$ einer ausführlichen Diskussion des allokativen Spielraumes einer Steuerreform von Oehsen (1983), insbesondere S. 24. 
eine Reform, bleibt Individuum B benachteiligt. Ideal wäre ein individuell differenziertes steuersystem, in dem beide ihr bisheriges steueraufkommen erbringen, aber Individuum B ein nach seinem Präferenzmuster differenziertes Verbrauchsteuersystem erhält.

Aus empirischer und finanzpolitischer sicht hätte ein solches Unterfangen keine Realisierungschance. Darüber hinaus 1 äßt sich unter institutionellen Aspekten zeigen, daB ein solches Steuersystem prinzipiell unerwünscht ist, weil es, abgesehen von der Chance für eine Pareto-Verbesserung, auch die Möglichkeiten für die totale Ausbeutung der Individuen durch den Staat zuläßt ${ }^{1}$ ).

Der vorgetragenen Kritik mag man entgegenhalten, daß diese strenge (theoretische) Auslegung des Pareto-Kriteriums keine sinnvolle Grundlage für eine praktische Reformpolitik ist, weil in der Realität jede Steuerreform sowohl Effizienz- als auch Verteilungsaspekte miteinander verbindet. Dem Einwand ist insoweit beizupflichten, als er darauf aufmerksam macht, daß Verteilungsargumente in der finanzpolitischen Diskussion häufig eine größere Rolle spielen als Effizienzaspekte. Im folgenden Kapitel werden deshalb ausführlich die Verteilungsargumente einer Steuerreform diskutiert. Indessen bleibt die Kritik an den theoretischen, informationstechnischen und praktischen Problemen des vorliegenden Modelis bestehen.

1) Vgl. dazu Kapitel $V$ dieser Arbeit 
Distributionsargumente sind nicht nur Teil jeder Steuerreformdebatte, sie sind meist sogar der eigentliche Motor der Steuerreformdynamik ${ }^{1)}$. Folgerichtig haben sie auch Eingang in die Theorie der optimalen Besteuerung (OT) gefunden. Und dies hat seinen guten Grund: Zwar läßt die OT aus finanzpolitischer Perspektive einige Wünsche offen, andererseits aber besteht einer ihrer größten Erfolge in der Präzisierung des Konflikts zwischen Effizienz- und Verteilungszielen. Im folgenden spielt die Illustration dieses Konflikts jedoch eine untergeordnete Rolle. Wichtiger erscheint die Diskussion um die Bedeutung alternativer Wohlfahrtsfunktionen als normative Basis der Verteilungszielsetzungen.

Allerdings kann in diesem Zusammenhang auf eine ausführliche, formale Abhandlung der Verteilungswirkung alternativer sozialer Wohlfahrtsfunktionen verzichtet werden, weil der Vergleich zwischen OT- und Leviathanregeln, der im folgenden Kapitel vorgenommen wird, zeigt, daß die Åquivalenz der Regeln nur für die Effizienzregeln der OT gilt. Die Verteilungsregeln der OT sind mit den Empfehlungen für den Leviathan nicht kompatibel und spielen deshalb in den folgenden Untersuchungen eine untergeordnete Rolle. Außerdem lassen sich die wesentlichen Modifikationen der Effizienzregeln, die aus den Verteilungszielsetzungen resultieren, bereits anhand einiger Beispiele veranschaulichen.

Im Vordergrund steht die Frage, ob und wie konsensfähige Verteilungsnormen abgeleitet werden können, die langfristig normkonsistente, d.h. verteilungsgerechte Steuerreformen ermöglichen. Wie im Ein-Konsumenten-Modell kann die Antwort nicht modellendogen, sondern nur auf politischer Ebene gegeben werden, weil dort die kollektiven Entscheidungen über Verteilungsnormen fallen. In den Verteilungsmodellen der OT wird diese Problematik aber explizit ausgeklammert. Statt dessen werden die Verteilungsnormen von einem exogenen Entscheidungsträger vorgegeben, um das Problem der

1) Vgl. auch Kap. VIII dieser Arbeit. 
Aggregation differierender Werturteile zu umgehen. In der Wohlfahrtsökonomie ist diese Vorgehensweise durchaus üblich. Ihr muß unzweifelhaft ein hoher theoretischer Erkenntniswert zuaeschrieben werden, der wesentlich zum Verständnis ökonomischer Zusammenhänge in der normativen Steuertheorie beiträgt. Aus finanzpolitischer sicht ist aber zu prüfen, ob nicht gerade infolge der Vernachlässigung der kollektiven Entscheidungsprozesse nur steuerpolitisch irrelevante Reformempfehlungen abgeleitet werden können ${ }^{1)}$. Auf diesen Problemkreis wird am Ende des Kapitels eingegangen.

\section{IV.1. OT-Empfehlungen im Mehr-Personen-Modell}

Das Ein-Konsumenten-Modell kann mit kleinen Modifikationen auf Personen erweitert werden ${ }^{2)}$. Die optimale steuerstruktur wird nun jedoch nicht mehr allein durch die Präferenzen der Konsumenten bestimmt, sondern auch durch die ihnen zugeordneten Wohlfahrtsgewichte. Die soziale Gewichtung der Präferenzen einzelner Gesellschaftsmitglieder erfolgt durch die Spezifikationeiner individualistischen, sozialen Wohlfahrtsfunktion (SWF), die dadurch gekennzeichnet ist, daß der gesellschaftliche Wohlfahrtsindex von den Nutzenindizes der einzelnen Gesellschaftsmitglieder abhängt. Eine individualistische SWF hat daher die Form ${ }^{3}$ ):

1) Damit ist u.a. auch auf das Arrow-Theorem hingewiesen, das besagt, daß es unter bestimmten, sachlich begründeten Bedingungen nicht möglich ist, die unterschiedlichen Wertvorsteliungen mehrerer Individuen zu einer logisch konsistenten Wertordnung dieser Individuen als Gruppe zu aggregieren. Siehe Arrow (1951), Sen (1970), Ng (1982), Kap. 5 oder Sohmen (1976), Kap. 10. Zu einer Kritik des Arrow-Problems siehe Little (1952) und Samuelson (1967). Die vielfältigen Versuche, dem Arrow-Dilemma zu entfliehen, zeigen seine überragende Bedeutung für Probleme kollektiver Entscheidungsprozesse. Siehe Z.B. Kemp/Ng (1976) oder Parks (1976)

2) Vgl. Atkinson/Stiglitz (1980), Kap. 14, Tresch (1981), S. $350 \mathrm{ff}$.

3) Gemeint sind hier Bergson-Wohlfahrtsfunktionen. Siehe Bergson (1938).

Diese Wohlfahrtsfunktion entspricht der Wertordnung eines einzelnen Individuums der Gesellschaft. Sohmen interpretiert diesen Sachverhalt folgendermaßen: "Der Begriff 'Wohlfahrtsfunktion' ist zunächst nichts weiter als eine Leerformel, die nicht mehr hergeben kann, als man ihr an einschränkenden Annahmen auferlegt." (S. 15) und "daß es sich bei der Manipulation mit Wohlfahrtsfunktionen, [...], nur um Gedankenexperimente eines bestimmten Betrachters handeln kann, von denen man kaum mehr erwarten darf als eine gewisse Hilfe bei der Ordnung und Disziplinierung seiner (eigenen) Gedanken". Sohmen (1976), S. 337 
(IV.1) $\quad W=W\left(v_{1}, \ldots, v_{H}\right)$.

Mit einer gegebenen sozialen Wohlfahrtsfunktion können alternative Steuersysteme sowohl nach Effizienz-als auch nach Verteilungsgesichtspunkten eindeutig bewertet werden. Die statliche Maximierungsaufgabe im Mehr-Personen-Modell lautet dann: Maximiere die gesellschaftliche Wohlfahrt unter der Nebenbedingung, da $B$ ein bestimmtes Steueraufkommen $\bar{T}$ realisiert wird ${ }^{1}$ ).

Sofern keine Informationsprobleme existieren und das Steuerinstrumentarium nicht eingeschränkt wird, sind differenzierte Pauschalsteuern optimal, die an den individuellen Fähigkeiten der Steuerzahler anknüpfen ${ }^{2}$. Die einfachste direkte Besteuerung würde also sowohl das Effizienz- als auch das Gerechtigkeitsziel ohne Allokationsverluste erfüllen.

Individuelle Fähigkeiten entziehen sich meist der objektiven Erfassung durch die Finanzbehörden. Differenzierte Pauschalsteuern sind daher technisch und politisch nicht durchsetzbar. Infolgedessen wird der Lohnsatz als Indikator für die individuellen Fähigkeiten empfohlen.

Würde man aus diesem Grunde das Steuerinstrumentarium und die Steuerbemessungsgrundlage derart einschränken, daß lediglich eine optimale nichtlineare Einkommensteuer (mit variablem Grenzsteuersatz) zulässig wäre, und würde man gleichzeitig unterstellen, daß die Nutzenfunktionen der Individuen schwach separabel zwischen Arbeit und Freizeit seien, so könnte das Effizienz- und Verteilungsziel ohne zusätzliche Steuern erreicht werden ${ }^{3)}$.

Die OT-Empfehiungen ändern sich drastisch, wenn die Einkommensteuer nur einen linear-progressiven Tarif aufweisen darf, und die

1) Vgl. Z.B. Atkinson/Stiglitz (1976), S. 60 f. Weitere grundlegende Beiträge stammen von Diamond/Mirrlees (1971b), Feldste in (1972) oder Boadway (1976). In der deutschsprachigen Literatur finden sich einige der wichtigsten Ergebnisse bei Rose/Wiegard (1983).

2) Vgl. Rose/Wiegard (1983), S. 90

3) Vgl. Atkinson/Stiglitz (1976), S. $67 \mathrm{f}$. 
Steuerbemessungsgrundlage auf alle Konsumgüter ausgedehnt wird. Besitzen die Nutzenfunktionen der Individuen nicht die (unrealistischen) Eigenschaften der Separabilität bzw. Ausgabenproportionalität, so muß die lineare Einkommensteuer und hier speziell der Pauschalsteueranteil für das Effizienzziel eingesetzt werden, während die differenzierten Verbrauchsteuern das Verteilungsziel weitmöglichst realisieren sollen ${ }^{1) 2}$ ).

Am Beispiel von Nahrungsmitteln und Luxusgütern lassen sich die ökonomischen Implikationen am leichtesten interpretieren ${ }^{3)}$. Gütergruppen werden von verschiedenen Einkommensklassen in unterschiedlichen Proportionen konsumiert. Die differenzierten Steuersätze verzerren die relativen Preise zwischen Nahrungsmitteln und Luxusguitern und treffen daher indirekt die verschiedenen Einkommensgruppen. Die Steuer auf Nahrungsmittel wird tendenziell das Realeinkommen der schlecht verdienenden Personen stärker reduzieren als das der Personen mit hohem Einkommen, die einen relativ geringen Teil der Nahrungsmittel konsumieren. Dementsprechend trifft die Steuer auf Luxusgüter die oberen Einkommensklassen stärker. Kann man nun den sozialen Grenznutzen des Einkommens verschiedener Einkommensgruppen gewichten (d.h. einen Grenzwert $\partial W / \partial v_{h}$ beimessen), so kann man auch die relativen Effekte der marginalen Anderungen der Steuer auf die soziale Wohlfahrt bewerten, weil die Einkommenseffekte der Steuern auf verschiedene Einkommensgruppen bewertbar werden ${ }^{4}$. Um die Verteilungswirkungen des Gutes einschätzen zu können, muß daher bekannt sein, in welchem Verhältnis es von verschiedenen Einkommensgruppen konsumiert wird und wie der soziale Grenznutzen des Einkom-

1) Vgl. Atkinson/Stiglitz (1980), S. 432

2) Verzichtet man gänzlich auf eine Einkommensteuer und vernächlässigt alle Kreuzpreiseffekte, so erhält man die erweiterte inverse Elastizitätsregel. Val. Z.B. Sandmo (1976), S. 49. Die Regel konterkariert die inverse Elastizitätsregel nach Effizienzgesichtspunkten (Kap. II. Gleichung II.44) um so mehr je stärker das Verteilungsziel berücksichtigt werden muß. Allerdings müssen die Umverteilungswirkungen reiner indirekter Steuern tendenziell sehr gering eingeschätzt werden. Vgl. dazu Sah (1983), S. 101

3) Vgl. auch Boadway/Wildasin (1984), S. 278

4) Formal entspricht diese Oberlegung der "distribution characteristic" eines Gutes. Vgl. Feldstein (1972), S. $32 \mathrm{ff}$. 
mens verschiedener Gruppen bewertet werden sol $1^{1}$. Wenn man bei steigendem Einkommen von einem abnehmenden sozialen Grenznutzen des Einkommens ausgeht, muß das Verteilungsziel in bezug auf Preis änderungen von Nahrungsmitteln verstärkt berücksichtigt werden. Ein Ansteigen der Preise für Nahrungsmittel würde daher die soziale Wohlfahrt eher reduzieren als ein Anstieg der Preise für Luxusgüter. Ceteris paribus müßten daher die Steuersätze auf Luxusgüter höher sein als jene auf Nahrungsmittel.

Während die Konsumgewohnheiten verschiedener Gruppen möglicherweise betrachtet werden können, ist die soziale Grenzbewertung des Konsums dieser Gruppen keine objektiv meßbare Größe, sondern resultiert aus einer vorgegebenen Wohlfahrtsfunktion, deren Entwicklung als modellexogener Entscheidungsprozeß aufgefaßt werden muß. Das optimale Steuersystem hängt von der Gestalt der SWF ab, die ihrerseits die Konkretisierung bestimmter Verteilungsnormen darstellt. Das Problem kann an der inversen Elastizitätsregel veranschaulicht werden ${ }^{2)}$. Der folgende Abschnitt hat den Zweck, die Wirkung unterschiedlicher Wohlfahrtsfunktionen auf das Ausmaß der Umverteilung zu illustrieren.

IV.2. Zur Umverteilungswirkung alternativer sozialer Wohlfahrtsfunktionen

Die Bedeutung alternativer Wohlfahrtsfunktionen für die Umverteilung soll in einem Zwei-Konsumenten-Modell graphisch veranschaulicht werden. In Abbildung 2 wird der Nutzen des Individuums A auf der Ordinate, der des B auf der Abszisse abgetragen. Abbildung 2 verdeutlicht, daß die endgïltige Nutzenverteilung auf einer willkürlich fixierten Nutzengrenze durch den Verlauf der Wohlfahrtsfunktion bestimmt wird.

Sofern die Gewichtung der Nutzen der Gesellschaftsmitglieder durch individualistische Wohlfahrtsfunktionen zum Ausdruck kommt,

1) Zur Ableitung von Wohlfahrtsgewichten siehe Harsanyi (1955) oder konkretere Vorschläge von Havemann (1965), Margl in (1967), Weisbrod (1968) oder McGuire/Garn (1969)

2) Vgl. Z.B. Sandmo (1976), S. $49 \mathrm{f}$. 
kann sie durch irgendeine Spezifikation der Funktion ${ }^{1)}$

(IV.2) W $=\left(\sum_{h=1}^{H} v_{h}^{\rho}\right)^{\frac{1}{\rho}}$

wiedergegeben werden, wobei $\rho$ eine konstante Größe darstellt, die als Indikator für die Aversion des wohlwollenden Planers gegenüber Nutzendifferenzen dient.

Die spezielle Ausprägung

(IV.3) W $\quad \sum_{h} v_{h}$

entspricht den klassischen utilitaristischen Vorstellungen von Bentham 2), die die Maximierung der Summe der individuellen Nutzen fordern. Sie kann mit $\rho=1$ aus (IV.2) gewonnen werden $^{3}$ ) und ist in Abbildung 2 als Gerade mit einer Steigung von -1 eingezeichnet. Eine SWF mit $\rho=1$ impliziert die gleiche gesellschaftliche frenzbewertung der Nutzen oder die völlige Indifferenz gegenüber Ungleichheit ${ }^{4}$ ). Sie tangiert $z$ wischen $B$ und $B^{\prime}$ unendlich viele Punkte, so da $B$ kein eindeutiges Wohlfahrtsoptimum bestimmt werden kann. Wenn $\rho$ unendlich groß wird, erhält man das Rawls'sche Maximim-Kriterium, welches die Maximierung der Wohlfahrt des am schlechtesten gestellten

1) Vgl. dazu Atkinson (1973), S. 94, Feldstein (1973), Feldstein (1976a), S. 80 , Bradford/Rosen (1976), S. 97 oder Atkinson/Stiglitz (1980). S. 340

2) Vgl. Bentham (1907) und Mill (1921)

3) Im folgenden wird das utilitaristische Prinzip im weitesten Sinne verstanden, so daß auch andere quasi-konkave wohlfahrtsfunktionen erlaubt sind. $\mathrm{Vgl}$. Feldstein (1976a), S. 80. Auch die dem Leistungsfähigkeitsprinzip zugrundeliegenden Opfertheorien können als Spezialfälle in eine soziale Wohlfahrtsfunktion integriert werden. Dies ist jedoch nur unter Einschränkungen möglich. Beispielsweise darf die Besteuerung keine Anderung des Sozialproduktes bewirken sowie keine Substitutionseffekte verursachen und entspricht damit einer Pauschalbesteuerung. Da das Leistungsfähigkeitsprinzip noch anderer schwerwiegender Kritik unterliegt, wird auf eine Diskussion in diesem Zusammenhana verzichtet. $\mathrm{Vgl}$. zu einer ausführlichen Diskussion und Kritik Pohmer (1970), Haller (1973/74), Littmann (1970), (1977), Schmidt (1980), Rose/Wiegard (1983), S. $54 \mathrm{ff}$.

4) Vgl. Tresch (1981), S. 25 
Individuums der Gesellschaft fordert ${ }^{1}$ ). Wie die Graphik zeigt, ist die Rawls'sche SWF durch eine rechtwinklige Form mit dem Optimalpunkt $R$ gekennzeichnet. Eine andere, beliebige Wahl des Parameters $\rho$ kann der Wohlfahrtsfunktion eine in vielen Graphiken abgebildete konkave Form mit dem optimalpunkt $W$ geben. Je nach der Parameterwahl wird sich der Verlauf der Wohlfahrtsfunktion und damit die gewünschte Nutzenverteilung ändern.

Wenn nur das optimale Güterbiundel $(\bar{z})$ effizient bereitgestellt wird und keine staatlichen Umverteilungsmaßnahmen ergriffen werden, könnte man sich $P$ als pareto-optimale Marktiösung vorstellen.

Schränkt der Staat sein Leistungsanaebot ein und gewährleistet lediglich Grundrechte sowie das Recht auf Vertragsfreiheit, wird beispielsweise nur Punkt $N$ auf der Nutzengrenze erreicht werden können ${ }^{2)}$. Diese Lösung erscheint jedoch unrealistisch, weil sich beide Individuen durch die zusätzliche Bereitstellung öffentlicher Güter bis zum Punkt $P$ verbessern können ${ }^{3)}$.

Eine andere extreme Gerechtigkeitsnorm ist das egalitaristische Prinzip, das Umverteilungsaktivitäten solange fordert, bis alle Individuen dasselbe Nutzenniveau erreicht haben. Es mißt daher der nutzenmäßgigen Gleichstellung aller Individuen per se die größte Bedeutung bei. Mit der Gleichverteilung der Nutzen wird beispielsweise Punkt $E$ in Abbildung 2 erreicht 4 ). Die Gleichverteilung aller Nutzen kann jedoch schwerwiegende Auswirkungen auf die Motivation der Individuen haben. Das Steuerinstrumentarium, das diese Verteilung erreichen soll, wird erhebliche Substitutionseffeke verursachen und vermutlich auch eine gravierende Reduktion der Leistungsbereitschaft der produktivsten Individuen. Das erarbei-

1) Siehe Rawls (1971), (1974) und zu einer Erweiterung des Prinzips Sen (1970), S. 138

2) Siehe Nozick (1974), insbesondere S. IX

3) Val. Atkinson/Stiglitz (1980), S. 337 f. bzw. Buchanan/Tullock (1962), S. 189 ff.

4) An diesem Beispiel erkennt man, daß das Rawls'sche Kriterium nicht zwangsläufig zu einer egalitären Verteilung führen muß. Vgl. Atkinson/Stiglitz $(1980)$, S. 341 
Abb.2 Ergebnisse alternativer Gerechtigkeitskriterien

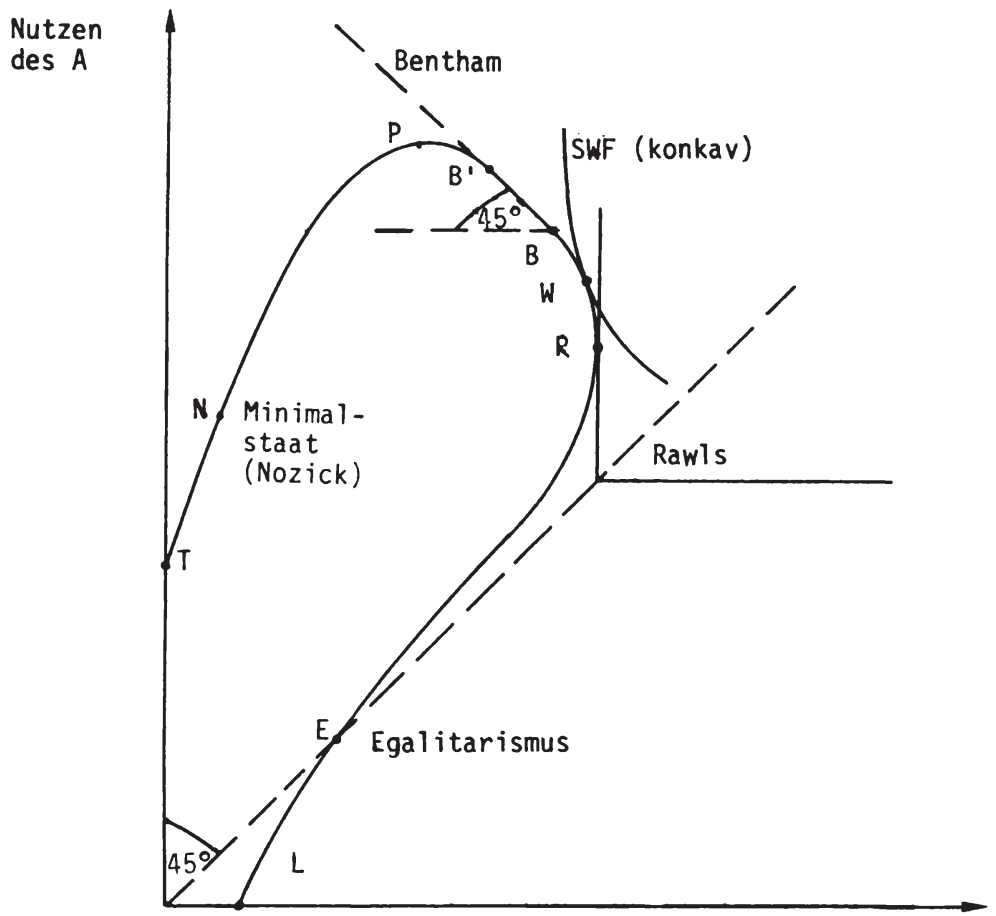

Nutzen des B

Abb. 2 beruht auf Fig. 11-1 in Atkinson/Stiglitz $(1980)$, S. 338 
tete Sozialprodukt ist nicht unabhängig von seiner Verteilung. Für Punkt E gilt ebenfalls, daß sich beide Individuendurcheine Reduktion der Umverteilungsmaßnahmen besserstellen können. Aus diesem Grund wird in der weiteren Untersuchung davon ausgegangen, daß die beiden Extrempositionen ( $E$ und $N$ ), diemit individualistischen Wohlfahrtskriterien nicht kompatibel sind, für demokratische Gesellschaftsordnungen kaum eine Rolle spielen werden.

Die aus Abbildung 2 resultierende Erkenntnis, daß sich je nach Wahl der Wohlfahrtsfunktion sehr unterschiedliche optimale Steuerinstrumente ableiten lassen, wird auch durch die Ergebnisse aus Simulationsmodellen bestätigt ${ }^{1)}$. Die Berechnungen von Harris/MacKinnon zeigen, daß durch die Einfühung der Wohlfahrtsfunktion $w=\frac{1}{\rho}\left(u_{A}^{\rho}+u_{B}^{\rho}\right)$ und bei unterschiedlichen Erstausstattungen zweier Individuen $A$ und $B$ die optimalen Steuersätze gegenüber der reinen Effizienzlösung (identische Individuen und Erstausstattung) stark abweichen. Insbesondere wird die Vermutung erhärtet, daß relativ unelastisch nachgefragte Güter (wie beispielsweise Nahrungsmittel) erheblich geringer besteuert werden sollten, wenn Verteilungsqesichtspunkte zu berücksichtigen sind. Die Variation der Parameterwerte $\rho$ (hier allerdings nur zwischen 0,1 und 0,9 ) hat keinen so bedeutsamen Einfluß auf die Steuersätze (im Beispiel maximal $6,9 \%)$; dies mag jedoch im wesentlichen an den unterstellten Stone-Geary-Nutzenfunktionen 1iegen ${ }^{2}$ ), deren postulierte Para-

1) $\mathrm{Vgl}$. dazu insbesondere die Arbeiten von Deaton (1977) und Harris/Mackinnon (1979). Weitere wichtige empirische Arbeiten bzgl. optimaler Einkommensteuern finden sich bei Mirrlees (1971), Atkinson/Stiglitz (1980), S. $421 \mathrm{f}$. oder Stern (1976). Einfache aber illustrative Beispiele für die Umverteilungswirkungen und die Sensitivität der Ergebnisse werden von Ricketts (1981). S. $30 \mathrm{ff}$. vorgeste11t.

2) Die Autoren selbst machen folgendermaßen darauf aufmerksam: "This may be in part because the utility function since it involves committet quantities, tends to benalize inequality regardless of the social welfare function. Or it may simply be that the demand system is very restrictive, so that there is no such scone for redistribution by altering tax rates." Harris/ Mackinnon (1979), S. 211. Vgl. dazu auch Atkinson/Stialitz (1980), S. $381 f$. Die Bedeutung der Parameterwerte $\rho$ in OT-Modellen und deren Sensitivität wird jedoch in Modellen optimaler Einkommensteuern, die andere Nutzenfunktionen unterstellen, eindrucksvoll bestätigt. Val. dazu Stern (1976), Tab. 3 
meterwerte ebenso wichtig für die Höhe der Steuersätze sind wie das von der Regierung geplante Steueraufkommen und die Ausgangsverteilung der individuellen Fähigkeiten oder ihrer Indikatoren (Löhne). Die numerischen Exempel von Harris/Mackinnon veranschaulichen die Sensitivität der optimalen Steuersätze in bezug auf alternative Zielfunktionen. Aus dieser Oberlegung ergibt sich:

\section{Steuerreformregel 4 :}

Vor jeder Steuerreform sind die gewünschten Verteilungsziele genau zu spezifizieren, da divergierende Gerechtigkeitsideale erhebliche Variationen der Steuersätze nach sich ziehen können.

Anhand des oben skizzierten Konflikts zwischen direkter und indirekter Besteuerung erlaubt die OT weitere qualitative Einsichten in die Effizienz- und Gerechtigkeitsstruktur eines optimalen Steuersystems ${ }^{1)}$. Es konnte festgehalten werden, daß es a priori keine Ziel-Mittel-Beziehung derart gibt, daß die Einkommensteuer den Gerechtigkeitszielen und die Verbrauchsteuer den Effizienzzielen der Besteuerung dienen müßten. Ob direkte oder indirekte Steuern zu präferieren sind, hängt vielmehr vom Steuerinstrumentarium, der Steuerbemessungsgrundlage und den unterstellten Nutzenfunktionen ab. Existieren keine finanzpolitischen oder steuertechnischen Restriktionen, dann ist eine einfache, an den individuellen Fähigkeiten gemessene Kopfsteuer die beste Besteuerungsform. Als wesentliche Voraussetzung für konsistente Steuerreformen folgt:

Steuerreformrege 1 5:

Vor jeder Steuerreform sind ein adäquater Modelirahmen, ein rea1 istisches Angebots- und Nachfragesystem, das Steuerinstrumentarium und die Verteilungszielsetzung genau zu spezifizieren, denn

1) Vgl. auch Little (1951). Zu einem allgemeinen Oberblick über neuere Erkenntnisse der Theorie der Besteuerung siehe Bradford/Rosen (1976), Stiglitz/Boskin (1977), Musgrave (1981b), Musarave (1983a), Atkinson/ Stiglitz (1980) oder Rose/Wiegard (1983) 
jede Anderung innerhalb dieses Datenkranzes führt in der Regel zu erheblichen Modifikationen der optimalen Steuerregeln. Mit anderen Worten, vor jeder Steuerreform sind alle Restriktionen und die zu maximierende Zielfunktion festzulegen.

Trotz der Evidenz dieser Regel wird ihren weitreichenden Implikationen in der finanzpolitischen Praxis vielfach keine genügende Bedeutung beigemessen. Aber auch bei gutem willen der Entscheidungsträger sind in der Realität kaum jemals die Voraussetzungen (insbesondere die Informationsanforderungen) erfiul1t ${ }^{1}$ ), die ein umfassendes Optimierungskalkül ermöglichen. So stellt sich die Frage, wie die auf individuellen Nutzen basierenden Prinzipien steuerli cher Gerechtigkeit in konkrete Steuergesetze transformiert werden können, solange keine exakte kardinale Nutzenmessung und keine interpersonellen Nutzenvergleiche möglich sind ${ }^{2)}$. Abgesehen von der Informationsproblematik bleibt ferner offen, inwieweit interpersonelle Nutzeninterdependenzen vernachlässigt werden sollen. Beispielsweise werden Nutzen aufgrund von Altruismus, Neid, Schadenfreude u.ä. aus moralischen Gründen üblicherweise vernachlässigt ${ }^{3)}$. Diese Berücksichtigung ethischer Restriktionen in den utilitaristischen Wohlfahrtsfunktionen verlangt nach neuen Werturteilen und legt gleichzeitig auch eine theoretische Schwäche des Ansatzes offen ${ }^{4}$. Abgesehen von den theoretischen Voraussetzungen einer Optimierungsaufgabe muß aber auch geprüft werden, ob eine individuelle Nutzenmessung im Auftrage des Fiskus, unter Berück-

1) Vgl. Z.B. Sohmen (1976), S. 436 f. oder Ng (1977), S. 1, die ausdrücklich auf die prohibitiven Informations - und Administrationskosten von SecondBest-Lösungen hinweisen.

2) Die Analyse kardinaler Nutzenmessung ist insbesondere mit Neumann/Morgenstern (1947) verbunden. Für einen überblick über die neueren Entwicklungen siehe Sen (1977) oder Boadway/Bruce (1984), Kap. 5.

3) Vgl. Z.B. Feldstein (1976a), S. 81; Altruismus und Verteilungsfragen bzw. Neid und Steuerprogression werden bei Hochman/Rodgers (1969) bzW. Bös/ Tillmann (1983) diskutiert.

4) Vgl. zu einer ausführlichen Diskussion weiterer Schwächen des wohlfahrtstheoretischen Ansatzes Sen (1979). Ein bisher unqelöstes Problem resultiert aus der Erkenntnis, daß horizontale Gerechtigkeit und die Maximierung einer SWF zu Zielkonflikten führen können. Val. dazu Atkinson/Stiglitz (1980), S. $13 \mathrm{ff}$., Stiglitz (1982), insbesondere Abschnitt 3 und Musgrave (1976) 
sichtigung aller individueller Umstände überhaupt wünschenswert ist. Zu diesem Zweck müssen die institutionellen Aspekte einer optimalen steuerreform näher untersucht werden, welche in der OT bisher beinahe völlig ausgeklammert wurden. Im folgenden Kapitel wird gezeigt, daß vollkommene Information über die individuellen Nutzenfunktionen der Steuerzahler zum Mißbrauch staatlicher Steuermacht beitragen kann. Die normative Steuertheorie kann daher bestenfalls als Versuchslabor verstanden werden, in dem nach optimalen steuerpolitischen Empfehlungen für alternative Idealökonomien (Rahmenbedingungen) und Wertvorstellungen gesucht wird ${ }^{1}$ ). Die Tatsache, daß neben neueren Gerechtigkeitsprinzipien der Besteuerung die traditionellen opfertheorien unter bestimmten Bedingungen in die Wohlfahrtsfunktion integriert werden können, beweist diesen Anspruch eindrucksvo 1 ${ }^{2}$ ). Höher gesteckte Erwartungen, wie zum Beispiel die Quantifizierung der einzelnen OT-Regeln, werden jedoch regelmäBig wegen prinzipieller theoretischer und empirischer Unzulänglichkeiten der OT-Modelle enttäuscht werden müssen ${ }^{3)}$.

1) Dabei können bereits a priori die Konsequenzen der Adaption spezifischer SWF zumindest in der Tendenz beurteilt werden. Ricketts (1981), S. $30 \mathrm{ff}$. macht in einem numerischen Beispiel die Schwierigkeiten deutlich, die aus der Verbindung gerechter Regeln mit gerechten Ergebnissen resultieren. Dabei geht er von drei Individuen mit identischen Nutzenfunktionen aus, die sich lediglich in ihren Fähigkeiten unterscheiden. Einkommen und Freizeit sind Argumente der Nutzenfunktion, die ihrerseits Argumente der sozialen Wohlfahrtsfunktion sind. Maximiert man nun die klassische Wohlfahrtsfunktion nach Bentham unter der Nebenbedingung einer gegebenen Produktionsfunktion, so erhält man gegenüber einer rein marktmäßigen Lösung recht kuriose Resultate. Beispielsweise müßte das am wenigsten produktive Individuum nicht mehr arbeiten, erhielte das doppelte Einkommen und das dreifache Nutzenniveau, während das produktivste Individuum weniger Einkommen erhielte, mehr arbeiten müße und erhebliche Nutzeneinbußen akzeptieren sollte. Obwohl man die Summe der individuellen Nutzen maximiert hat, handelt es sich hier tatsächlich um eine Sklavenhaltergesellschaft. Die Ergebnisse sind zwar im Bentham'schen Sinne optimal, ob jedoch Regeln akzeptiert werden, die zu diesen Ergebnissen führen, bleibt zu bezweifeln. Vgl. zu dieser Diskussion auch Kap. V. Abs. 3, dieser Arbeit.

2) Vgl. Hahn (1973), S. 106. Der OT fällt daher, wie bereits erwähnt, eine bescheidenere, aber immer noch wesentliche Rolle zu.

3) Albers bezeichnet die "Optimierungsversuche" gar als Irrwege. Albers (1977), S. 126. Diese Einschätzung hält Wiegard für voreilig, Wiegard (1982), S. 161. Eine scharfe (und wahrscheinlich überzogene) Verurteilung der Wohlfahrtsökonomik findet sich auch bei Woll (1984), S. 32 f. 


\section{IV.3. Fazit}

Das vorliegende Kapitel demonstriert, daB optimale Steuerregeln neben dem Steuerinstrumentarium, der Steuerbemessungsgrundlage und individuellen Präferenzen auch von gesellschaftlichen Gerechtigkeitsnormen abhängen, die sich durch eine soziale Wohlfahrtsfunktion spezifizieren lassen. Im Mehr-Personen-Modell müssen daher nicht nur die in Kap. II beschriebenen Informationsprobleme gelöst werden, vielmehr ist die Wahl einer sozialen Wohlfahrtsfunktion (bzw. Verteilungsnorm) eine zusätzliche Voraussetzung für eine umfassende Optimierung.

Jede Anderung der relativen Verteilungsgewichte zwischen den Bevölkerungsgruppen führt daher zu Modifikationen der OT-Regeln. Die naheliegende Forderung, da $B$ mit jeder Variation der Rahmenbedingungen eine neue optimale Reformstrategie oder gar eine neue optimale Steuerstruktur konzipiert werden sollte, muB nicht nur als völlig unrealistisch zurückgewiesen werden, sie ist auch ineffizient, weil die hohen Reformkosten aller Steuerzahler, die Reformgewinne einzelner i.d.R. übersteigen ${ }^{1}$ ).

Die Schaffung normkonsistenter, langfristiger Zielvorgaben ist jedoch, ebenso wie die Durchsetzung optimaler Steuerregeln, von der Funktionsfähigkeit des politischen Prozesses abhängig. In der OT werden jedoch mit der Hypothese des allwissenden, wohlwollenden Alleinherrschers die politisch-institutionellen Aspekte von Steuerreformen ausgeklammert, obwohl gerade sie in der steuerpolitischen Praxis die entscheidende Rolle spielen. Denn schlieblich entscheiden die politischen Vorgaben über Verteilungsziele oder Steuersatzrestriktionen daruber, welche Reformstrategie oder welches Steuersystem optimal ist. Im folgenden ist daher zu klären, welche Rolle die politischen Institutionen und Entscheidungsmechanismen für die Durchsetzung optimaler Steuerreformen spielen.

1) I.d.R. werden Steuerrechtsänderungen und Ungerechtigkeiten mit sich bringen. Vgl. Hochman (1974) und Kap. VII dieser Arbeit. 
V. INSTITUTIONELLE ASPEKTE VON STEUERREFORMEN UND KONSTITUTIONELLE STEUERREGELN

Ein gravierender Einwand gegen die traditionelle normative Steuertheorie, insbesondere aber gegen die OT, richtet sich gegen die Vernachlässigung wesentlicher institutioneller Aspekte ${ }^{1)}$. Der Vorwurf zielt vor allem auf die unrealistischen, wenn nicht gar inkonsistenten Annahmen über das Verhalten der politischen Entscheidungsträger. Obwohl in den OT-Modellen nutzenmaximierende Konsumenten und gewinnmaximierende Produzenten unterstelltwerden, besteht die Regierung aus einem wohlwollenden und allwissenden Diktator, der in selbstloser Weise ein gesellschaftliches optimum realisiert. Ein Blick in Geschichte und Gegenwart lehrt, daB dieses Bild politischer Entscheidungsmechanismen in keiner Weise der Realität entspricht.

An dieser Stelle könnte man entgegnen, daß es nicht Aufgabe der normativen Theorie sei, ein bestimmtes Politikerverhalten zuanalysieren; statt dessen soll die OT Aussagen darüber liefern, wie sicheine Regierung verhalten sollte, wenn sie eine optimale Steuerreform im Sinne vorgegebener finanzpolitischer Ziele realisieren will. Diese Sichtweise hat jedoch in Hinblick auf praxisrelevante Steuerreformempfehlungen nur dann Berechtigung, wenn man überhaupt von monolithischen Entscheidungen und gutwilligen politischen Institutionen ausgehen könnte, die in der Lage wären, optimale Steuerregeln im Sinne der postulierten Ziele umzusetzen. Leider zeigt die Erfahrung, daß diese Voraussetzungen eher die Ausnahme als die Regel sind. Die Unzufriedenheit gegenüber ungerechtfertigten Entscheidungen des Fiskus kommt sowohl durch wachsenden Bürgerprotest ${ }^{2)}$ als auch durch zunehmende kritik in der

1) Vg1. Z.B. Littmann (1977), S. 104. Er kritisiert die große "Diskrepanz zwischen den Handlungsvorschriften der normativen Theorie und dem konkreten Verhalten der staatlichen Planer". Dadurch verkümmert der "normative Ansatz zu einer esoterischen oder gar sterilen Konstruktion."

2) Beispielhaft dafür steht die Steuerzahlerrevolte in den USA. Vgl. dazu Z.B. Citrin (1978), Oakland (1979), Kuttner (1980), Ladd/Tideman (1981), Folkers (1983a). Benkert (1983). In der Bundesrepubl ik Deutschland wird der Bürgerprotest durch den Bund der Steuerzahler organisiert und artikuliert. Vgl. z.B. Bund der Steuerzahler (1983) 
wissenschaftlichen Diskussion ') zum Ausdruck. Akzeptiert man diesen Einwand nicht, muB man sich den Vorwurf gefallen lassen, Steuerregeln für das Paradies ableiten zu wollen, deren aktuelle Realisierung prinzipiell nicht möglich ist und - unter bestimmten Bedingungen - auch nicht erwïnscht wäre.

Eine institutionelle Theorie von Steuerreformen muB sich daher vorrangig mit der Frage beschäftigen, welche Reformaktivitäten aus dem politischen Entscheidungsprozeß typischerweise zu erwarten sind. Durch die positive Fundierung der institutionellen Steuertheorie lassen sich dann möglicherweise institutionelle Regelnableiten, die den Gesetzgeber zu normkonsistentem Handeln zwingen. Institutionelle Regeln sind somit als Restriktionen staticher Institutionen, hier speziell von steuerinstitutionen, zu verstehen.

Das optimale Funktionieren von Steuerinstitutionen ist Voraussetzung für optimale Steuerreformen bzw. Steuersysteme. Die folgende Analyse zeigt, daB OT-Regeln ohne die Berücksichtigung der Ausgabenseite des Budgets nicht in jedem Falle wïnschenswerte Ergebnisse liefern, weil die Gefahr besteht, daß ausgerechnet die praktikabelsten Effizienzregeln für eigennützige politische Zielsetzungen miBbraucht werden können. Das Dilemma, daß unterschiedliche Motivationen statlichen Handelns mit ein und derselben Steuerregel kompatibel sind, wird insbesondere im Zusammenhang mit der konstitutionellen Steuertheorie von Brennan/Buchanan ${ }^{2}$ diskutiert. Um der Problematik unkontrollierter Steueraufkommenserhöhungen zu begegnen, schlagen Brennan/Buchanan daher fiskalische Restriktionen für den Fiskus vor. Eine Analyse ihrer konstitutionellen Begrenzungsvorschläge zeigt aber, daß konkrete Beschränkungen der Steuerbemessungsgrundlage oder der Steuersätze nur im Falle eines Steuerstaates ökonomisch gerechtfertigt erscheinen, der das Steueraufkommen zu maximieren wüscht. Schließlich werden die normative und politische Basis der konstitutionellen und normativen Steuertheo-

\footnotetext{
1) Vgl. zur aktuellen Diskussion Z.B. Neumark (1982), (1983) oder Littmann (1984)

2) Vgl. Brennan/Buchanan (1980)
} 
rie gegenübergestellt und analysiert. Dabei wird erneut deutlich, da $B$ insbesondere die Empfehlungen quantitativer, konstitutioneller Steuerregeln von alternativen Erwartungen über das potentielle Verhalten des Fiskus im laufenden politischen ProzeB abhängen.

V.1. Alternative Hypothesen über die Verhaltensweise des Regierungssektors im steuerreformprozeB

Steuerpolitische MiBstände werden in der Regel staatlichem Versagen angelastet. Würde fiskalisches und gesellschaftliches Interesse übereinstimmen, dirfte es eigentlich keine steuerpolitisch motivierten Proteste geben 1). Es ist also naheliegend, die Divergenz zwischen Wählerinteressen und Steuerstaat in den unterschiedlichen Zielsetzungen beider Gruppen zu suchen. Ein erster Schritt zu einer realistischeren Einschätzung fiskalischer Steuerreformentscheidungen besteht in der Ablehnung der Hypothese eines "wohlwollenden Planers". Erst die Integration alternativer Hypothesen über das Verhalten von politischen Institutionen erlaubt Aussagen darüber, welche Steuerreformregeln im politischen Spektrum zu erwarten sind und inwieweit diese Regeln von jenen der optimalen Besteuerung abweichen. SchlieBlich erhält man auf diese Weise ersten Aufschluß darüber, wie Institutionen beschaffen sein müssen, damit Abweichungen von optimalen Steuerreformprogrammen minimiert werden.

\section{V.1.1. Der Leviathan-Steuerstaat}

Um die Konsequenzen eigennütziger staatlicher Zielsetzungen klarer isolieren zu können, wird vorerst noch ein monolithischer Regierunassektor unterstellt, der seine steuerpolitischen Vorstellungen unbeeinfluBt formulieren und verfolgen kann, Dieses Bild staatijcher (Steuer-) Machtausubung deckt sich mit den institutionellen Imolikationen des OT-Konzeptes (val. Abb. 1), die der Realität nicht entsprechen. Wie gezeigt werden kann, existieren nicht nur direkte Handlungsanweisungen der Regierung, sondern vielfältige

1) Vgl. Folkers (1983a), S. $62 \mathrm{ff}$. 
Einflüsse von Seiten der Bürokratie, Wähler und Interessengruppen, die zu erheblichen Modifikationen der vom Fiskus ursprünglich intendierten Steuerregeln führen können. DaB zunächst trotzdem das einfache Bild eines Alleinherrschers beibehalten wird, liegt darin begründet, daß die Ziel-und Handlungskategorien der einzelnen Reformbeteiligten auf diese Weise eindeutig herausgearbeitet werden können.

Wie aber soll die neu zu konzipierende Zielfunktion des Staates formuliert werden? Um den Kontrast zur Hypothese eines "wohlwollenden Planers" in der OT am anschaulichsten demonstrieren zu können, sollten die potentiellen negativen Handlungsweisen des Fiskus durch eine Verhaltenshypothese beschrieben werden, die gleichzeitig als Basis für eine weitere positive Analyse fiskalischer Aktivitäten dienen kann. Diese Vorgehensweise erlaubt es, diejenigen Steuerregeln zu identifizieren, die der Bürger im schlimmsten Fall zu erwarten hat. Auf der Grundlage dieser Ergebnisse können schließlich steuerpolitische Empfehlungen - genauer fiskalische Verfassungsregeln - abgeleitet werden, die die unerwünschten Auswüchse schlechter Institutionen verhindern sollen ${ }^{1}$.

Will man die genannten Anforderungen an die Zielfunktion berücksichtigen, treten vor allem zwei Eigenschaften staticher Steuermacht in den Vordergrund: Erstens besitzt der Staat ein verfassungsrechtlich verankertes Finanzmonopol (vgl. z.B. Art. 105 GG der Bundesrepublik Deutschland) und zweitens wird die Gesetzgebungskompetenz von nutzenmaximierenden Politikern wahrgenommen. Eine problematische Verbindung beider Funktionen ergibt sich dann, wenn man unterstellt, daß der Nutzen der finanzpolitischen Entscheidungsträger mit dem Niveau ihrer Gehälter, sowie mit anderen im Amt gewährten Vergünstigungen und diese wiederum mit der Höhe des Steueraufkommens eindeutig positiv korreliert sind. Ohne zusätzliche Verfassungsschranken könnte der Staat seinen monopolistischen Spielraum in der Steuergesetzgebung analog zum privaten Monopol für einige

1) Vgl. Brennan/Buchanan (1980), S. 26 
Zwecke einsetzen ${ }^{1}$ ). Unter diesen Voraussetzungen kann die Fiktion eines Staates, dessen Ziel es ist, das Steueraufkommen zumaximieren, im folgenden kurz Leviathan ${ }^{2}$ genannt, als erste Annäherung an ein realistischeres Bild staticher Steuerpolitik verstanden werden.

Diese These wurde bereits vor einigen Jahren in der deutschsprachigen Literatur diskutiert ${ }^{3)}$. In jüngster Vergangenheit wurde sie insbesondere von Brennan/Buchanan ${ }^{4}$ in den Mittelpunkt der Steuerreformdiskussion gestellt. Dabei nehmen Brennan/Buchanan für sich in Anspruch, mit Hilfe der Leviathanthese aktuelles, in der Realität beobachtbares Regierungsverhalten zu beschreiben ${ }^{5}$. Der Auffassung von Brennan/Bucharian wird hier nur insoweit gefolgt werden, als auch den Mitgliedern des Regierungsapparates Nutzenmaximierung unterstellt werden muß.

Der Leviathan wird als die unerwüschteste Steuerinstitution interpretiert, deren Steuerregeln zusammen mit den OT-Regeln die Bandbreite potentiellen statichen Verhaltens darstellen. Steueraufkommensmaximierung wird daher nicht als typische Verhaltenswe ise aktueller Regierungen verstanden ${ }^{6)}$, sondern im Sinne einer

1) Vgl. zu einer umfassenden Rechtfertigung des Leviathanmodells Brennan/ Buchanan (1980), S. $13 \mathrm{ff}$. Die Problematik wird aber auch in Abschnitt V.2. nochmals aufgenommen.

2) Der Begriff "Leviathan" stammt aus dem Hebräischen ( 1 iwjatan = Der Gewundene) und bezeichnet ein drachenartiges Ungeheuer, das sogar Sonne und Mond zu verschlingen trachtet. Die Begriffsbestimmung im hier verwendeten Sinne geht auf Hobbes (1651) zurück, vgl. Buchanan (1975), S. 147

3) Vgl. Gandenberger (1966)

4) Die These vom böswilligen, ausbeutenden Diktator geht auf frühere Arbeiten der italienischen Autoren de Viti, de Marco zurück. Vgl. Brennan/ Buchanan (1980), S. 15 und die dort in Fußnote 1 angegebene Literatur. Nicht zitiert werden dort die Arbeiten von Gandenberger (1966) und (1975/76).

5) "At this level of discourse, our analysis is necessarily more positive. We introduce models of how governments do behave or how they may be predicted to behave (regardless of the advice that they may be advanced by public finance economics)." Brennan/Buchanan (1980), S. 1

6) Tatsächlich relativieren auch Brennan/Buchanan (1980) im Laufe ihrer Arbeit die Bedeutung dieser Verhaltenshypothese für die positive Analyse des aktuellen Regierungsverhaltens. 
äuBerst negativen Referenzsituation ${ }^{1)}$. Es ist nun zu prüfen, welche Steuerregeln ein nur durch ökonomische Vorgaben restringierter Leviathan verfolgen würde, wie sie sich von den OT-Ergebnissen unterscheiden, und ob das zu erwartende Verhalten des Steuerstaates in der Realität beobachtbar ist.

\section{V.1.2. Das Mode 11}

Um die Konsequenzen des Leviathanverhaltens isoliert herausarbeiten zu können, wird das eingangs formulierte OT-Modell, abgesehen von kleineren technischen Modifikationen ${ }^{2}$, mit lediglich einer wesentlichen Anderung übernommen: Die statliche Zielsetzung besteht nun darin, das Steueraufkommen zu maximieren. Analog wie in Kap. II wird unterstellt, daß der repräsentative Steuerzahler seinen Nutzen unter der Nebenbedingung seiner Budgetrestriktion zu maximieren sucht.

(v.1) $\max u(x)$ u.d.N. $q_{i} x_{i}=m \quad i=1, \ldots, n$

Setzt man die daraus ableitbaren (normalen) Marktnachfragefunktionen in die Budgetrestriktion des Haushalts ein, so folgt:

1) In diesem Zusammenhang sei darauf hingewiesen, daß staatliche Verteilungsaktivitäten möglicherweise zu noch niedrigeren Nutzenniveaus der Steuerzahler führen können, als es durch die Steueraufkommensmaximierung des Leviathan möglich wäre. Das gilt z.B. im Falle der Gleichverteilung (Egalitarismus). Die Problematik resultiert aus der Tatsache, daß diese nichtindividualistische Verteilungsnorm keine individuellen Substitutionseffekte und daher auch nicht die Motivationsstruktur der Individuen berücksichtigt, sondern nur die Gleichverteilung als solche anstrebt. In diesem Falle wäre also mit einem sehr niedrigen Sozialprodukt und einem entsprechend geringen Steueraufkommen zu rechnen ( $\mathrm{vgl}$. auch Abb. 2 in Kap. IV). Es ist daher denkbar, daß in einem Leviathanstaat mehr ökonomische Aktivitäten durchgeführt werden als in einem Staatswesen, das eine sehr gravierende Einkommensumverteilung anstrebt.

2) Im Gegensatz zu Kap. II wird hier vom primalen Optimierungsansatz ausgegangen, obwohl dies für die Ergebnisse ohne Bedeutung ist. Vgl. für die Herleitung des identischen staatlichen Optimierungsergebnisses Anhang D. Die Modifikation ist allein deshalb sinnvoll, weil sie einerseits eine einfachere ökonomische Interpretation zuläßt und andererseits den Vergleich mit den Ergebnissen in Abschnitt VI.3.3. erleichtert. 
(v.2) $\sum_{i=1}^{n} a_{i} x_{i}(q)=m$.

Im Produktionssektor werde wiederum effizient produziert, so daß die langfristigen Gewinne der Unternehmen bei konstanten Produzentenpreisen null werden:

(v.3) $\sum_{i=1}^{n} p_{i} y_{i}=0$.

Der Staat produziere ein vorgegebenes öffentliches Güterbündel $\left(\bar{z}_{\text {min }}\right)$ in eigener Regie, da man von der oberlegung ausgeht, da $B$ ein Minimum an Gegenleistung für die eingezogenen Steuern erbracht werden mu ${ }^{1}{ }^{1}$. Der wahre Leviathan zeigt sich allerdings darin, daß er mit Hilfe des maximierten Steueraufkommens private Güter und Faktoren aus privater Verwendung für eigene Zwecke zu absorbieren sucht. Das maximale Steueraufkommen $T^{\max }$ muß daher im Systemgleichgewicht den mit Marktpreisen bewerteten Güterund Faktorkäufen entsprechen:

(v.4) $\quad T^{\max }=p_{z} z+\sum_{i=1}^{n} p_{i} y_{i}^{G}$.

Es sollen abgesehen von Kopfsteuern lediglich Gütersteuern untersucht werden, so da $\beta$ es sich anbietet, die konsumenten- und Produzentenpreise so $z u$ normieren, daB der Faktor Arbeit $\left(x_{1}\right)$ unbesteuert bleibt und daher als numéraire $\left(t_{1}=0\right)$ dient. Für alle übrigen Güter gilt dann:

(v.5) $\quad a_{i}-p_{i}=t_{i} \quad i=2, \ldots, n$.

SchlieBlich müssen noch die Marktgleichgewichtsbedingungen

$$
x_{i}=y_{i}+y_{i}^{z}+y_{i}^{G} \quad i=1, \ldots, n
$$

1) Vgl. Brennan/Buchanan (1980), Kap. 2 
erfüllt sein, damit alle modellrelevanten Bedingungen formuliert sind. Gemäß dem Walras-Theorem $1 a ̈ B t$ sich aus den Gleichungen $(V .2),(V .3),(V .5)$ und $(V .6)$ explizit die Budgetgleichung des Staates ableiten ${ }^{1)}$ :

(v.7) $\quad \sum_{i=2}^{n} t_{i} x_{i}(a)-m=\sum_{i=1}^{n} p_{i} y_{i}^{z}+\sum_{i=1}^{n} p_{i} y_{i}^{G}=T$.

Einziges Ziel des allwissenden Leviathan ist die Maximierung des Steueraufkommens, wobei er die Reaktionen der Steuerzahler auf die Anderung der Marktdaten in seinen Kalkuil einbeziehen muß. Wenn der Staat bei vollkommener Information über Preis- und Mengenreaktionen in der Lage ist, $T z u$ maximieren, und zudiesem Ziel alle Steuersätze beliebig variiert, kann er als "eingeschobener Doppelmonopolist"2) interpretiert werden. Dieser Leviathan greift weder in den ProduktionsprozeB ein, noch hat er Steuerverwaltungs- oder Erhebungskosten ${ }^{3)}$. Auf der Angebots- und Nachfrageseite sehen sich die statichen Instanzen polypolistischen Verhaltensweisen gegenüber. Da der Leviathan im Falle fixer Produzentenpreise ausschließlich die Konsumentenpreise manipuliert, entspricht seine "Steuerabsatzfunktion" dem Ausdruck

(v.8) $\quad x_{i}=x_{j}(\bar{p}+t)$,

der wie die inverse Preisabsatzfunktion eines Monopolisten interpretiert werden kann. Der Einfachheit halber und um die hier re-

1) Durch Einsetzen von (V.5) und (V.6) in (V.3) folgt:

$\sum_{i=1}^{n}\left(a_{i}-t_{i}\right)\left(x_{i}-y_{i}^{z}-y_{i}^{G}\right)=m\left(\right.$ mit $\left.t_{i}=0\right)$.

Subtrahiert man davon (V.2), d.h.

$\sum_{i=1}^{n} q_{i} x_{i}-\sum_{i=2}^{n} t_{i} x_{i}-\sum_{i=1}^{n} q_{i} x_{i}=\sum_{i=1}^{n} p_{i} y_{i}^{z}+\sum_{i=1}^{n} p_{i} y_{i}^{G}+m$, so erhäit man (V.7).

2) Vgl. dazu Gandenberger (1975/76), insbesondere S. 409

3) Vgl. zu den Problemen der Steuertechnik oder Finanzkontrolle z.B. Schäfer (1977). 
sultierenden Ergebnisse mit jenen der OT vergleichen zu können, sei von konstanten Produzentenpreisen $\bar{p}$ ausgegangen, so da $B$ der Leviathan den Charakter eines reinen Angebotsmonopolisten auf weist ${ }^{1}$. Der Ausdruck

(v.9) $\quad \sum_{i=2}^{n} p_{i} x_{i}(a)$

entspricht der Kostenfunktion des Leviathan, wenn man davon aus geht, daß der Gesamterlös $\sum a_{j} x_{j}(a)$ zunächst dem Fiskus zufließt, der dann die mit fixen Produzentenpreisen bewertete Nachfrage im Sinne von Kosten (entgangenem Gewinn) an die Unternehmen weiterleitet. Die Budgetrestriktion des Leviathan (V.7) kann folglich als die zu maximierende Steuergewinnfunktion interpretiert werden ${ }^{2}$ ).

Nach der Beschreibung der Modellgrundlagen können nun die Steuerregeln für den Leviathan abgeleitet werden.

\section{V.1.3. Die Steuerreqeln des Leviathan}

Gemäß (V.7) lauten die Bedingungen erster Ordnung für ein Steueraufkommensmaximum:

(v.10) $\quad \frac{\partial T}{\partial q_{k}}=\sum_{i=2}^{n} t_{i} \frac{\partial x_{i}}{\partial q_{k}}+x_{k}=0 \quad k=2, \ldots, n$

und

(v.11) $\quad \frac{\partial T}{\partial m}=\sum_{1=2}^{n} t_{i} \frac{\partial x_{i}}{\partial m}-1=0$.

Unter Berücksichtigung der Slutsky-Gleichung

1) Vgl. dazu auch Gandenberger (1966), S. 1 ff.

2) $\mathrm{Vgl}$. Gandenberger (1975/76), insbesondere S. $406 \mathrm{f}$. 
(v.12) $\frac{\partial x_{i}}{\partial q_{k}}=s_{i k}-\frac{\partial x_{i}}{\partial m} x_{k}$

wird $(v .10) \mathrm{zu}$

(v.13) $\sum_{i=2}^{n} s_{i k} t_{i}=-x_{k}+\sum_{i=2}^{n} t_{i} \frac{\partial x_{i}}{\partial m} x_{k}$.

Erweitert man (V.11) mit $x_{k}$ und setzt das Ergebnis in (V.13) ein, so folgt:

(v.14) $\quad \sum s_{i k} t_{i}=0$.

Daraus resultiert:

Leviathan-Rege 1 :

Im Maximum sollten alle Steuerbeträge $t_{i}$ null sein und die Steuern ausschlieBlich durch maximale Kopfsteuern erzielt werden.

Der wesentliche Unterschied zur OT-Regel besteht somit nicht in der Art, sondern in der Höhe der Kopfsteuern. Pauschalsteuern als solche sind in der OT die effizientesten Steuern - gleiches gilt aus der Sicht des Leviathan ${ }^{1)}$. Sie sind definitionsgemäß unabhängig von ökonomischen Größen und erlauben daher einen direkten Steuerzugriff, ohne Konsequenzen auf ökonomische Verhaltensweisen fürchten zu müssen. Abstrahiertman von der (unrealistischen) Modellvorstellung, welche besagt, daß der Leviathan immer das Minimum an Leistungen erbringe, das ihm die Herrschaft sichert, so erhebt sich die Frage, ob sich die Steuerzahler durch eine Pauschalsteuer völlig ausplündern ließen, zumal eine an kein ökonomisches Kriterium gebundene Steuer gegen traditionelle Gerechtigkeitsvorstellungen verstößt, so daß über kurz oder lang auch

1) $\mathrm{Vgl.zu}$ einer graphischen Ableitung des Ergebnisses Brennan/Buchanan.(1977), S. 261 
machtpolitische Konsequenzen für den Leviathan vorstellbar wären ${ }^{1)}$. Dies gilt insbesondere dann, wenn die Kopfsteuer hart an das Existenzminimum heranreicht und jede ökonomische Aktivität, die Einkommen erbringt, zur Sinnlosigkeit verurtei1t $t^{2}$. Aspekte des Ungehorsams von Steuerzahlern, wie z.B. die Substitution legaler durch illegale Einkünfte aus der Schattenwirtschaft, werden in diesem Modell ebensowenig berücksichtigt wie in den OT Modellen. Beide Modellkonzeptionen beinhalten nur ökonomische Restriktionen (Budgetrestriktionen, vgl. Abb. 1) und sind daher im Grunde unpolitische Ansätze, deren Regeln gleichermaßen schwierig in die Realität umzusetzen sind. Der politisch-ökonomische Ansatz unterstellt keine rein passiven Reaktionen der Steuerzahler auf die ausbeutenden Steuerregeln des Leviathan. Eher ist zu erwarten, daß die Zensiten über direkte oder indirekte Wege aktiv in den steuerpolitischen Entscheidungsprozeß eingreifen ${ }^{3)}$. Aus dieser Sicht ist es sehr ungewiß, bis zu welcher Aufkommenshöhe die effizienztheoretischen Pluspunkte einer Kopfsteuer erhalten bleiben. Denn gerade die offenkundige Ungerechtigkeit von aufkommensmaximalen Pauschalsteuern und die Tatsache, daß sie an keine ökonomischen Kriterien anknüpfen, erschwert die Einschätzung, wann Konterreaktionen der Zensiten eintreten. Es scheint jedoch klar, daßein Leviathan diese Steuereinkommensquelle soweit wie möglich nutzen wird.

Diese Empfehlung folgt aber auch aus den OT-Modellen eines wohlwollenden Diktators. Die bisher vermutete Vorteilhaftigkeit von Pauschalsteuern muß also bezweifelt werden. Sie hängt entscheidend von der Zielfunktion des finanzpolitischen Entscheidungsträgers ab. Gerade dieses Beispiel zeigt, wie wichtig eine positive Fundierung von politischen Institutionen für die Aussagekraft der normativen Steuertheorie ist. Ist es daher aufgrund dieser Er-

1) Aspekte des Ungehorsams von Steuerzahlern oder Steuerzahlerrevolten (wie in den USA geschehen) werden bei Brennan/Buchanan (1980) als demokratisches Mittel zur Bekämpfung des Leviathan kaum beachtet.

2) Dabei wird unterstellt, daß keine Naturalabgaben an den Staat geleistet werden müssen. Somit können auch Kopfsteuern nur aus Gewinn, Einkommen oder Bestandsgrößen (Vermögen) finanziert werden.

3) Vgl. dazu Kap. VII dieser Arbeit. 
kenntnisse sinnvoll, Kopfsteuern überhaupt als Steuerinstrumente in Erwägung $z u$ ziehen $^{1)}$ ? Diese Frage soll im AnschluB an die Ergebnisse des folgenden Abschnittes beantwortet werden.

Um weitere Zusammenhänge zum OT-Modell herzuleiten, sei davon ausgegangen, da $B$ der Leviathan aus irgendwelchen Gründen keine Kopfsteuer erheben und der Einfachheit halber nur die Verbrauchsteuern variieren kann. Die für den Leviathan optimale Steuerstruktur resultiert aus (V.12). Wegen der symmetrie der Substitutionseffekte und unter Berücksichtigung von

(v.15) $\quad k=\sum_{i=2}^{n} t_{i} \frac{\partial x_{i}}{\partial m}-1$

folgt aus $(V .10)$ :

(v.16) $\frac{\sum_{i=2} t_{i} s_{k i}}{x_{k}}=k$.

Leviathan-Regel 2:

Gleichung (V.16) entspricht genau der OT-Regel (II.35). Aus (V.16) folgt, daB der Leviathan und der wohlwollende Planer im jeweiligen Optimum dieselben Steuerregeln einhalten sollten ${ }^{2}$.

Ebenso wie im Drei-Güter-Fall von Corlett/Haque kann nun deutlich gemacht werden, daß ein zur Freizeit komplementäres Gut höher besteuert werden sollte als ein Gut, das zur Freizeit in Substitutionsbeziehung steht. Dies ist ökonomisch einleuchtend, da statt eines hoch besteuerten Freizeitsubstitutes lieber Freizeit konsu-

1) In OT-Modellen wird eine Steuer, die an den individuellen Fähigkeiten und an der maximal zur Verfügung stehenden Arbeitskraft ankriüpft als optimal bezeichnet. Vgl. Rose/Wiegard (1983). S. 90. Diese einer Pauschalsteuer entsprechende Einkommensteuer wäre im Sinne des Leviathan aber ein optimales Ausbeutungsinstrument und daher nicht zu empfehlen.

2) Dieses Ergebnis wurde in anderer Weise bei Brennan/Buchanan (1980), S. $80 \mathrm{ff}$. oder bei Blankart/Bongaerts (1981), S. $14 \mathrm{ff}$. abgeleitet. 
miert würde, die jedoch kein Steueraufkommen erbrächte. Der Leviathan wird daher solche Substitutionsvorgänge durch eine mög1 ichst hohe Besteuerung von freizeitkomplementären Gütern zu verhindern suchen. Eine einfacher zu interpretierende Steuerregel erhält man, wenn die Kreuzpreiseffekte der verschiedenen Angebotsund Nachfragekurven vernachlässigt werden. Die modifizierten Bedingungen erster ordnung lauten dann:

(v.17) $\quad \frac{\partial T}{\partial q_{i}}=\frac{\partial x_{i}}{\partial q_{i}} t_{i}+x_{i}=0$.

Leviathan-Rege 1 3:

Aus (V.17) ergibt sich die bereits bekannte inverse Elastizitätsrege ${ }^{1}$ )

$$
\frac{t_{i}}{p_{i}}=\tau_{i}=-\frac{1}{\varepsilon_{i i}} \text {, }
$$

die besagt, daß diejenigen Güter am höchsten besteuert werden sollten, die die geringste (prozentuale) Nachfrageänderung aufgrund einer (einprozentigen) Preisänderung aufweisen.

Die Ergebnisse zeigen, daß die Effizienzregeln der OT und die Leviathanregeln unter gleichen ökonomischen Voraussetzungen von ihrer struktur her identisch sind.

\section{V.1.4. Die Konsequenzen der Maximalbesteuerung}

Die Gleichungen (V.16) und (V.18) bestätigen, daß die Effizienzregeln der OT mit den Leviathanregeln übereinstimmen. Die effizienztheoretischen Empfehlungen der optimalen Steuertheorie wüden daher den Wüschen eines einkommensmaximierenden Leviathans tendenziell entsprechen, allerdings mit dem Unterschied, daB $T^{\max }$ normalerweise größer als $\bar{T}$ sein wird. Das bedeutet, daß

1) Vgl. Kap. II, Gleichung (II.44). 
von der Steuerstruktur allein nicht mehr auf das tatsächliche Regierungsverhalten rückgeschlossen werden kann. Nur der Beweis. daß $\bar{T}$ kleiner $T^{\max }$ und $T^{\max }$ tatsächlich die obergrenze des erzielbaren steueraufkommens ist, impliziert steueraufkommensmaximierung im umfassenden sinne ${ }^{1}$ ).

$T^{\max }$ setzt also voraus, daß der Leviathan eine umfassende Steuerbemessungsgrundlage und das komplette Steuerinstrumentarium einsetzen wird. Diese Zielrichtung deckt sich exakt mit den steuerpolitischen Ratschlägen der OT. In Kap. II wurde nämlich gezeigt, daß jede Einschränkung der Steuerbemessungsgrundlage und des Steuerinstrumentariums ( $z . B$. das Verbot von Pauschalsteuern) zu suboptimalen bzw. Second-Best Ergebnissen führt. Die gesellschaftlichen Wohlfahrtsverluste rühren somit nicht aus der Art der Besteuerung, sondern aus der eigennützigen Absorption oder Verschleuderung von Steuergeldern, die der Differenz zwischen maximalem und optimalem Steueraufkommen entsprechen. Die Vernachlässigung der Ausgabenseite des Budgets macht deutlich, daß OT-Empfehlungen allein keine Garantie für eine umfassende, optimale Lösung sind.

OT-Effizienzregeln sind also nur dann eindeutig vorteilhaft, wenn der Fiskus tatsächlich ein optimales Steueraufkommen erzielen möchte. Dagegen sind sie für den Leviathan eine notwendige Voraussetzung, um die Zensiten perfekt ausbeuten zu können. Der Zusammenhang belegt, daß wohlgemeinte Steuerratschläge ohne die Kontrolle der exekutierenden Steuermacht erhebliche Gefahren für die Zensiten bergen. Daraus ergibt sich:

Steuerreformregel 6 :

Unter der Voraussetzung von unbeschränkten, eigennutzorientierten Steuerinstitutionen kann der beliebige Einsatz von Steuerinstrumenten (umfassende Steuerbemessungsgrundlage, Pauschalsteuern)

1) Gemeint ist, daß in diesem Falle tatsächlich die umfassendste Steuerbemessungsgrundlage gegeben sein muß, d.h. im vorliegenden Modell definieren alle Güter und Faktoren die Steuerbemessungsgrundlage, die dem Leviathan als Entscheidungsbasis dient. Vgl. auch Brennan/Buchanan (1980), Kap. 3 
nicht empfohlen werden. Statt dessen bedarf es kontrollierbarer Restriktionen der Steuermacht des Leviathan. Das impliziert im Sinne der OT suboptimale Regeln, die das Steueraufkommen ( $z$. B. durch Beschränkungen der Steuerbemessungsgrundlage und der Steuersätze) limitieren. Diese Steuerregel steht in eindeutigem Widerspruch zu Steuerregel 1.

Hält man also den Leviathan für eine realistische Gefahr, müßte eigentlich gerade die Einhaltung von OT-Regeln verhindert werden, um der perfekten Ausbeutung zu entgehen. Brennan/Buchanan haben daher versucht, fiskalische Verfassungsregeln abzuleiten, die rationale Steuerzahler auf konstitutioneller Ebene wählen würden, um sich in den Folgeperioden vor dem Zugriff des Leviathan zu schützen. Im folgenden sollen zunächst die Logik sowie die Funktion konstitutioneller Steuergrenzen und im Anschluß daran ihre ökonomischen wirkungen näher untersucht werden.

\section{V.2. Die Logik fiskalischer Verfassungsrege In}

Ein permanent spürbares Machtinstrument des Staates besteht in seinem Recht, Steuern zu erheben. Das Leviathanmodell veranschaulicht, daß dieses Machtpotential nur schwer kontrollierbar ist, aber leicht mißbraucht werden kann. "Seen in this way the power to 'tax' is simply the power to 'take'"1).

Brennan/Buchanan schlagen daher vor, die Aktivitäten des Leviathan durch eine Steuerverfassung zu begrenzen ${ }^{2}$ ), die einen Grundkonsens (fast) aller Steuerzahler repräsentiert. Einstimmige Entscheidungen auf konstitutioneller Ebene stellen das Kriterium für

1) Vgl. Brennan/Buchanan (1980), S. 8

2) Vgl. Brennan/Buchanan (1977), Brennan/Buchanan (1978) und Brennan/ Buchanan (1980). Die aktuelle Bedeutung von Steuergrenzen wird durch die Proposition 13 in Kalifornien vom 6.Juni 1978 dokumentiert. 
eine gute Verfassungsregel dar ${ }^{1}$. Die Entscheidungen auf der Verfassungsebene haben den Charakter von Entscheidungen über Spielregeln (konstitutionelle Entscheidungen) im Gegensatz zu Wahlhandlungen innerhalb eines Spiels, dessen Spielregeln gegeben sind (periodische Entscheidungen). Brennan/Buchanan unterstellen in der konstitutionellen Situation, daß die Wirtschaftssubjekte ähnlich der Rawls'schen Idee ${ }^{2)}$, hinter dem Schleier der Unwissenheit ${ }^{3)}$ ihre eigene Position als Steuerbürger nicht identifizieren können. In dieser Situation müssen die Individuen Steuerrege $n$ und Institutionen (Spielregeln) wählen, die die Aktivitäten eines Leviathan in den postkonstitutionellen Perioden, d.h. im politischen Alltag, auf ein gewünschtes Maß begrenzen und gleichzeitig auf Dauer in Kraft bleiben. Der Verfassunascharakter fiskalischer Regeln dient daher sowohl dem Staat als auch den Steuerzahlern als langfristige Orientierungs - und Entscheidungshilfe. Nach Brennan/Buchanan besteht die Notwendigkeit fiskalischer Verfassungsregeln keineswegs nur bei der Existenz eines Leviathan, sondern auch in demokratischen Staatswesen, da die in den publicchoice-Modellen unterstellte Bereitstellung öffentlicher Güter gemäß den Präferenzen des Medianwählers oder seines Repräsentaten eine zu naive Darstellung aktiver Regierungspolitik darstellt ${ }^{4}$.

1) Vg1. Buchanan (1979), S. 351. Dieses Kriterium "To define as 'good' that which emerges from agreement among free men, independently of instrinsic evaluation of the outcome itself" wurde vor allem in Buchanan (1975), "The Limits of Liberty", entwickelt. Im Gegensatz hierzu sind die ethischen Normen der OT exogen gegeben (Effizienz-Distribution).

2) Vgl. Rawls (1971), Brennan/Buchanan (1979), S. 11

3) Nach Brennan/Buchanan (1977), S. 255 genügt es bereits, daß die Individuen höchst unsicher über ihre eigene zukünftige Position sind. Der wesentliche Unterschied zur Rawls'schen Idee liegt allerdings darin, daß die Individuen nicht gleich sein müssen und der Abstimmungsprozeß selbst das Entscheidungskriterium impliziert. Zur Analyse der Struktur des social contract siehe Buchanan (1977), S. $81 \mathrm{ff}$.

4) Brennan/Buchanan (1977), S. 258: "Confronted with public sectors of modern scope [...] the democratic-limits model of government fiscal constraint seems to become increasingly naive". Trotz des tiefen Mißtrauens gegenüber staatlicher Macht (The Power to Tax) befuirworten Brennan/Buchanan staatliche Zwangsgewalt. Dazu gehört auch das staatliche Steuermonopol, um das free-rider-Problem bei der Bereitstellung öffentlicher Giuter zu umgehen. 
Wie noch ausführlich analysiert werden wird, sind Mehrheitswahlsysteme keineswegs eine Garantie dafür, daß die Wählerpräferenzen angemessen zur Geltung kommen. Nur in einem Fallekönnten Wahlprozesse eine vollständige Kontrolle der Regierung garantieren: bei Einstimmigkeit; diese Regel spielt jedoch in der politischen Praxis aus gutem Grunde kaum eine Rolle ${ }^{1)}$.

Der Vorteil fiskalischer Verfassungsregeln besteht nun darin, daß sie durch einfache Mehrheit nicht außer kraft gesetzt werden können, so daß der politische Handlungsspielraum effektiv beschränkt wird ${ }^{2}$. Darüber hinaus erwartet man von ihnen, daß sie als letzte Bastion gegen Politikversagen und ausufernde Budgets fungieren, wenn die Kontrolle durch den Wahlprozeß und andere Verfassungsregeln nicht gewährleistet ist ${ }^{3}$ ). Die vom Bürger an die Regierung abgetretene Steuer- und Ausgabenmacht muß wirkungsvoll restringiert werden, ohne jedoch die notwendige Flexibilität fiskalischer Entscheidungen zu zerstören. "Die Logik konstitutioneller fiskalischer Restriktionen besteht somit darin, mögliche Unvollkommenheiten des politischen Systems in bezug auf finanzwirtschaftliche Entscheidungen zu kompensieren und von den Bürgerwünschen abweichende Ergebnisse so weit als möglich zu verhindern"4). Diese Neuorientierung des normativen Ansatzes besteht in den Entwurf einer Verfahrenslehre für den politischen Prozeß der Steuerbestimmung anstelle der bisher üblichen Ergebnislehre optimaler Steuerstrukturen ${ }^{5}$.

Die Vertreter der Leviathanhypothese sehen den Machmißbrauch durch einen viel zu großen, zu schnell wachsenden und ineffizienten Staatssektor belegt ${ }^{6)}$. Dementsprechend wurden vielfältige fiskalische Re-

1) Vgl. Brennan/Buchanan (1980), S. 6

2) Vgl. hierzu Brennan/Buchanan (1979), S. 12 ff., Brennan/Buchanan (1980), Kap. 1, Brennan (1981), S. $121 \mathrm{ff}$.

3) Siehe Z.B. das Problem zyklischer Mehrheiten. Downs (1957), Kap. 10

4) Folkers (1983a), S. 30

5) Folkers (1983b), S. 190

6) Zur Kritik siehe Musgrave (1981a) 
ge In für die Ausgaben- ${ }^{1)}$ und Einnahmenseite sowie für ein effizienteres Haushaltswesen entworfen. In bezug auf das Leviathanmodell interessieren vorrangig konstitutionelle Beschränkungen der Macht der Besteuerung, wenngleich sie mit Ausgabenbegrenzungen und Budgetierungsverfahren in einem engen Zusammenhang stehen und nicht immer eindeutig voneinander zu trennen sind ${ }^{2}$ ). Welche fiskalischen Verfassungsregein sich aus dem Leviathanmodell ableiten lassen und welche ökonomischen Wirkungen von ihnen ausgehen, ist Gegenstand der folgenden Analyse.

V.3. Konstitutionelle Steuerregeln von Brennan/Buchanan

Nachdem die Logik konstitutioneller Steuerregeln dargelegt wurde, erhebt sich die Frage, welche fiskalischen Regeln von rationalen Individuen auf konstitutioneller Ebene verabschieden würden, wenn in den Folgeperioden die Gefahr eines Leviathan existiert.

V.3.1. Prozedurale und ergebnisbezogene fiskalische Verfassungsrege $1 n$

Brennan/Buchanan nennen grundsätzlich zwei Kategorien fiskalischer Restriktionen, die die unerwünchten Aktivitäten des Leviathans korrigieren sollen ${ }^{3)}$ :

a) prozedurale fiskalische Beschränkungen

b) ergebnisbezogene fiskalische Beschränkungen.

ad a) Verfahrensmäßige oder prozedurale fiskalische Beschränkungen versuchen, "durch veränderte Regeln des finanzwirtschaftli-

1) Spezifische Ausgabengrenzen als Pendant zu den Steuergrenzen fordert z.B. Wildavsky (1980). Ein effizientes Haushaltswesen soll durch verschiedene Budgetierungsverfahren wie PPBS, ZBB oder durch die neuerdings diskutierte sunset legislation erreicht werden. Vgl. dazu Z.B. Langner (1983), Wildavsky (1975), Folkers (1983a)

2) Vgl. Folkers (1983a), S. 23 ff. und Buchanan (1980a), S. $83 \mathrm{ff}$.

3) Vgl. dazu Brennan/Buchanan (1980), S. $192 \mathrm{ff}$., Buchanan (198Ca), S. 83 ff. und zu einer Systematik fiskalischer Beschränkungen Folkers (1983a), S. 32 ff. 
chen Entscheidungsprozesses auf erwünschte Eraebnisse hinzuwirken"1). Zu diesen Ansätzen zählen z.B. ${ }^{2}$ ):

- Integration von Steuer- und Ausgabenentscheidungen;

- Zweckbindung öffenticher Einnahmen;

- Separation der unterschiedlichen fiskalischen Funktionen und der einzelnen Ebenen des föderativen Staates;

- materieller Haushaltsausgleich;

- spezielle Mehrheiten für Steuer- und Ausgabenentscheidungen;

- sunset legislation;

- Forderung nach einer proportionalen Einkommensteuer.

Einige der prozeduralen Regeln werden im Zusammenhang mit dem politisch-ökonomischen Steuerreformmodell noch ausfürlich diskutiert ${ }^{3}$ ).

ad b) Ergebnisbezogene fiskalische Beschränkungen "wirken direkt auf die Ergebnisse selbst ein, indem sie bestimmte Forderungen in Form von Höchstgrenzen festlegen, die von den politischen Entscheidungsträgern nicht überschritten werden dürfen"4). Dazu gehören quantitative Restriktionen, die "das Aufkommen einzelner Steuern oder des gesamten steuersystems, Steuerbemessungsgrundlagen und Steuersätze betreffen" 5 ). Ihnen kommt im Rahmen des Leviathanmodells besondere Bedeutung $z u$, weil sie dem Ziel der Steueraufkommensmaximierung unmittelbar entgegenwirken. Sie steht daher im Mittelpunkt der folgenden Analyse.

1) Folkers (1983a), S. 43

2) Vgl. Folkers (1983a), S. 33

3) Vgl. Kap. VII dieser Arbeit.

4) Folkers (1983a), S. 43

5) Folkers (1983a), S. 44 


\section{V.3.2. Die Modellgrundlage}

Die wichtigsten ergebnisbezogenen Begrenzungsregeln von Brennan/ Buchanan 1) können aus den Ergebnissen des vorliegenden Leviathanmodells abgeleitet werden.

Um die Steuerzahler ruhig zu halten, wird vom maximalen Steueraufkommen $T^{\max }$ ein gewisser Anteil $\alpha T^{\max }$, mit $0<\alpha<1$, zu Finanzierungen öffentlicher Güter und Dienste verausgabt, wobei $\bar{\alpha}$ exogen gegeben sei. Das restliche Steueraufkommen fließt dem Einkommen des Leviathans zu.

Unter diesen Voraussetzungen werden rationale Individuen auf der konstitutionellen Ebene das Steuerinstrumentarium, die Steuerbemessungsgrundlage und die Steuersätze derart beschränken, bis in den Folgeperioden

$$
\text { (V.19) } \quad \bar{\alpha} T^{\star}=Z^{*}
$$

realisiert wird, d.h. bis das maximal erzielbare Steueraufkommen gerade ausreicht, um ein optimales Bündel öffentlicher Güter $Z^{*}$ zu finanzieren. T* bezeichnet also das Steueraufkommen, das bei optimalen fiskalischen Restriktionen vom Leviathan maximal erzielt werden kann. Wenn $T^{\max }$ für das Maximalaufkommen steht, das der Leviathan ohne geeignete Restriktionen erheben könnte, d.h. Wenn $T^{\max } z . B$. den maximalen Kopfsteuern in $V .1 .3$. entspricht, so gilt:

$(v .20) \quad \bar{\alpha} T^{\star}<\bar{\alpha} T^{\max }$,

"since it is inconceivable that anyone could ever anticipate an 'efficient' public private sector mix that would require all potential income above subsistence for government purposes" 2 ). Die folgenden Begrenzungsregeln beschäftigen sich daher mit der Frage, wie $T^{*}$ angesichts eines Leviathans erreicht werden kann.

1) Vgl. Brennan/Buchanan (1980), S. $37 \mathrm{ff}$.

2) Brennan/Buchanan (1977), S. 262 
V.3.2.1. Begrenzungen des Steuerinstrumentariums und der Steuerstruktur.

Zunächst kann man festhalten, daB entsprechend Steuerreformregel 5 auch aus (V.19) folgt, daB Z.B. Kopfsteuern prinzipiell verboten werden müßten, eine Empfehlung, die den OT-Regeln vollkommen widerspricht. Um maximale Kopfsteuern erheben zu können, bedarf es einer umfassenden Steuerbemessungsgrundlage, die sowohl das monetäre Einkommen als auch die monetären Aquivalente der "nonmarket production of valued end-products" 1 ) enthält. Für den künftigen Steuerzahler wäre es daher rational zumindest das nicht-marktmäBig bewertete Einkommen als Steuerbemessungsgrundlage auszuschlieBen. Auch diese Empfehlung widerspricht den OT-Regeln, da die alleinige Besteuerung der monetären Einkommensströme zu Verzerrungen gegenüber den Freizeitaktivitäten des Zensiten und damit zu einer Mehrbelastung führt.

Aber, selbst bei AusschluB von Kopfsteuern und bei ausschließlicher Besteuerung monetärer Einnahmen-oder Ausgabenströme ${ }^{2)}$ bleibt dem Staat ein sehr groBes Ausbeutungspotential erhalten. Beispielsweise kann er durch die Einführung einer ideal regressiven Verbrauchsteuerstruktur die gesamte Konsumentenrente der Steuerzahler abschöpfen. Zu diesem Zweck müßte er für jede nachgefragte Gütereinheit den maximalen Steuerbetrag ermitteln, der so beschaffen sein müßte, daß der Kaufpreis (Produzentenpreis plus Steuerbetrag) für die jeweilige Gutseinheit nur geringfügig unter der maximalen Zahlungsbereitschaft für diese Gutseinheit liegen würde. Der rationale Steuerzahler würde sich daher auch Beschränkungen für die Steuersatzstruktur wünschen und regressive Steuern verbieten ${ }^{3)}$. Erlaubte man der Regierung nur einen proportionalen Steuersatz für alle Gütereinheiten auf einem Markt, aber differenzierte Steuersätze für

1) Brennan/Buchanan (1977), S. 261

2) Es sei nochmals daran erinnert, daß in diesem Modell z.B. von der Ersparnisund Vermögensbildung abstrahiert wird, wenngleich auch diese Größen einer marktmäBigen Bewertung zugänglich sind und in der aktuellen Diskussion um Steuergrenzen eine entscheidende Rolle spielen. Vgl. z.B. zur Diskussion um Proposition 13 Folkers (1983a), S. 151.

3) Vgl. Brennan/Buchanan (1977), S. 262 
verschiedene Märkte, so würde die optimale Steuerstruktur für den Leviathan exakt der Regel (V.16) entsprechen. Die Forderung nach einer allgemeinen Verbrauchsteuer mit einem auf allen Märkten gleichen Steuersatz hat deshalb automatisch eine Senkung des Steueraufkommens gegenüber einer differenzierten Verbrauchsbesteuerung zur Folge. Eine zusätzliche Minderung des Steueraufkommens ließe sich durch bestimmte progressive steuerstrukturen erreichen. Am Beispiel einer indirekt progressiven Einkommensteuer (= proportionaler Tarif mit einem fixen Freibetrag) beweisen Brennan/ Buchanan diese Behauptung, die analog auch auf Gütermärkte mit Iinearen Nachfragefunktionen übertragen werden kann. Ausgangspunkt der oberlegungen sind die Bedingungen erster Ordnung für ein Steueraufkommensmaximum auf einem Markt ohne Substitutionsoder Komplementärbeziehungen ${ }^{1)}$ :

(v.17) $\quad \frac{\partial T}{\partial t}=\frac{\partial x}{\partial q} t^{\star}+x^{\star}=0$,

wobei $t$ * den maximalen proportionalen Steuersatz auf alle Guitereinheiten dieses Marktes darstellt und * die im Maximum realisierten Größen kennzeichnet. Ohne die Berücksichtigung von Kreuzpreiseffekten gilt. für die Gütermengenänderung $\Delta x$ aufgrund der Steuererhebung:

$$
\Delta x=\frac{\partial x}{\partial q} \Delta q=\frac{\partial x}{\partial q} t \text {, mit } \Delta q=t \text {, }
$$

so daß statt (V.17) auch

(V.22) $\Delta x^{*}+x^{*}=0$

bzw.

( V.23)

$$
x_{i}^{\star}-x^{0}=-x^{*}
$$

und daher

1) Diese Annahme wird später aufgegeben. 
(v.24) $\quad x^{*}=\frac{1}{2} x^{0}$ oder $\Delta x^{*}=-\frac{1}{2} x^{0}$

geschrieben werden kann.

Das maximale steueraufkommen entspricht somit:

(v.25) $\quad T^{*}=t^{*} \Delta x^{*}=\frac{1}{2} t^{*} x^{0}$

und wegen (V.24) gilt:

(v.26) $\quad t^{*}=-\frac{x^{*}}{\frac{\partial x}{\partial q}}=\frac{1}{2} \frac{\frac{x^{0}}{q} q}{\frac{\partial x}{\partial q}}=\frac{1}{2 \varepsilon^{0}} q$,

so daB folgt:

(v.27) $\quad T^{*}=\frac{1}{4 \varepsilon^{0}} q x^{0}$, mit $\varepsilon^{0}=\frac{\Delta x^{0}}{x^{0}} / \frac{\Delta q}{q}$.

Im Falle einer indirekt progressiven Steuersatzstruktur auf demselben Markt hätte der statliche Optimierungsansatz die Form:

(v.28) $\quad T_{E}=t_{E}\left(x(q)-x_{E}\right)=t_{E} x(q)-t_{E} x_{E}$,

wobei $x_{E}>0$ den fixen Freibetrag symbolisiert. Die Bedingungen erster Ordnung für ein Maximum lauten:

(v.29) $\quad \frac{\partial T_{E}}{\partial t_{E}}=\frac{\partial x}{\partial q} t_{E}^{\star}+x^{\star}-x_{E}=\Delta x^{\star}+x^{\star}-x_{E}=0$.

Durch Einsetzen von

(v.30) $\quad \Delta x^{*}=\left(x^{*}-x_{E}\right)-\left(x^{0}-x_{E}\right)$

in (V.29) folgt: 
$(v .31) \quad\left(x^{*}-x_{E}\right)-\left(x^{0}-x_{E}\right)+x^{*}-x_{E}=0$

bzw.

(v.32) $\left(x^{\star}-x_{E}\right)=\frac{1}{2}\left(x^{0}-x_{E}\right)$.

Analog zur Berechnung von (V.26) resultiert aus (V.29) und (V.32) der maximale Steuersatz

$(\mathrm{V} .33)$

$$
\begin{aligned}
& t_{E}^{*}=\frac{\left(x^{*}-x_{E}\right)}{x^{*}} \cdot \frac{1}{\varepsilon^{\star}} q=\frac{1}{2} \frac{\left(x^{0}-x_{E}\right)}{x^{0}} \cdot \frac{1}{\varepsilon^{0}} q, \\
& \text { mit } \varepsilon=\frac{\Delta x^{0}}{x^{0}} / \frac{\Delta q}{q},
\end{aligned}
$$

und das maximale Steueraufkommen

$$
T *=t *\left(x^{\star}-x_{E}\right)=t * \frac{1}{2}\left(x^{0}-x_{E}\right)=\frac{1}{4} \frac{\left(x^{0}-x_{E}\right)^{2}}{x^{0}} \cdot \frac{1}{\varepsilon^{0}} q \cdot
$$

Aus einem Vergleich von $t^{\star}$ und $t_{E}^{\star} b z w . T^{*}$ und $T_{E}^{\star}$ ergeben sich die Beziehungen:

(v.35) $\quad t_{E}^{*}=\frac{1}{2} \frac{x^{0}-x_{E}}{x^{0}} \cdot \frac{1}{\varepsilon} q<\frac{1}{2} \frac{1}{\varepsilon} q=t^{*}$

bzw.

(V.36) $\quad T_{E}^{*}=\frac{1}{4} \frac{\left(x^{0}-x_{E}\right)^{2}}{x^{0}} \cdot \frac{1}{\varepsilon^{0}} q<\frac{1}{4} x^{0} \cdot \frac{1}{\varepsilon^{0}} q=T^{\star}$,

womit die obige Behauptung bewiesen wäre ${ }^{1}$ ).

1) Vgl. zu diesem Ergebnis Brennan/Buchanan (1977), S. 265 
Progressive Steuerstrukturen bewirken also i.d.R. eine stärkere Beschränkung des maximalen Steueraufkommens als proportionale Tarife $^{1)}$ und sind daher als Verfassungsregel zum Schutz vor dem Leviathan prinzipiell geeignet ${ }^{2}$ ).

\section{V.3.2.2. Einheitliche Steuerstrukturen für alle Steuerzahler}

Bisher wurden die maximierenden Steuerstrukturen nur in bezug auf ein repräsentatives Individuum abgeleitet. Tatsächlich gibt es aber sehr verschiedene Präferenzstrukturen, weshalb der Leviathan zur perfekten Realisierungeines Steueraufkommensmaximums auch zwischen den Steuerzahlern selbst differenzieren müßte ${ }^{3)}$. Jeder Zensit würde dann ganz individuell ausgebeutet. Infolgedessen bedeutet eine einheitliche Steuerstruktur für alle Individuen eine effektive Beschränkung des staatlichen Steuermonopols. Die Forderung nach einem gleichen Steuerrecht für alle impliziert insbesondere die Gleichbehandlung von Individuen in der gleichen ökonomischen Situation, nicht aber eine gleiche Steuerlast für alle. Die Forderung nach horizontaler Gerechtigkeit, die in der normativen Steuerlehre auf einem ethischen Postulat beruht, wird nun mit einem rationalen Entscheidungskalkül der Bürger auf konstitutioneller Ebene begründet. Steuersysteme, die nach individuellen Präferenzen differenzieren, sollten deshalb verfassungsrechtlich verboten $\operatorname{sein}^{4}$ ).

\section{V.3.2.3. Zur Wahl der Steuerbemessungsgrundlage}

Akzeptiertmandie bisher abgeleiteten Verfassungsregeln, die auch

1) Die Aussage erhält man auch im Falle linear ansteigender Grenzsteuersätze. Vgl. Brennan/Buchanan (1980), S. $52 \mathrm{ff}$. Brennan/Buchanan belegen aber auch, daß diese Ergebnisse nicht prinzipiell auf beliebige Progressionstarife übertragen werden können, wenn man verschiedene Individuen bzw. Präferenzstrukturen berücksichtigen muß. In diesen Fällen kommt es auf die individuellen Nachfrageelastizitäten an, ob das maximale Steueraufkommen größer oder kleiner als T* wird. Vgl. Brennan/Buchanan (1980), S. $49 \mathrm{ff}$.

2) Vgl. Brennan/Buchanan (1977), S. 266

3) Vgl. dazu die analoge Optimierunasstrategie der OT in Abschnitt III.2.

4) Vgl. Brennan/Buchanan (1980), S. 68 
aus der Sicht der traditionellen Steuerlehre kaum abzulehnen wären, so erhebt sich die Frage, welche Regeln zusätzlich in Kraft treten sollten, um das Steueraufkommen auf das gewünschte Niveau zu reduzieren, sofern dies notwendig sein sollte.

Nach Brennan/Buchanan sollte sich die Wahl dieser Regeln an dem Effizienzkriterium orientieren, d.h. wenn zwei Steuerstrukturen mit verschiedenen Begrenzungsregeln dasselbe maximale steueraufkommen erbringen, so ist jene Struktur zu präferieren, welche die geringste Mehrbelastung in den Folgeperioden erzeugt ${ }^{1}$ ). In bezug auf das vorliegende Leviathan-Modell mit einer differenzierten Güterbesteuerung kommen Brennan/Buchanan zu dem Ergebnis, daB im Falle linearer Nachfragefunktionen, unabhängig vom Umfang der Steuerbemessungsgrundlage, die Mehrbelastung die Hälfte des maximalen Steueraufkommens beträgt.

Das Ergebnis $1 \ddot{3}$ t sich im einfachsten Fall mit Hilfe der Gleichungen

$(v .26) \quad t^{*}=\frac{1}{2 \varepsilon^{0}} \mathrm{q}$

und

(V.25) $\quad T^{*}=\frac{1}{2} t * x^{0}$

bewe isen.

Unter der Voraussetzung linearer Kurvenverläufe entspricht die Mehrbelastung $\Delta W$ dem Harberger-Dreieck unterhalb der Nachfragefunktion:

(V.37) $\Delta W=\frac{1}{2} t^{\star} \Delta x *$. Im Maximum gilt:

1) Beispielsweise verursacht bei gleichem maximalem Steueraufkommen ein progressiver Steuertarif (mit Ausnahme der indirekten Progression) rege1mäBig eine höhere Mehrbelastung als eine proportionale Steuerstruktur. Vgl. Erennan/Buchanan (1977), S. $266 \mathrm{ff}$. 
$(V .38) \quad \Delta W^{\star}=\frac{1}{2} t^{\star} \Delta x^{*}$

und wegen (V.24) folgt:

(V.39) $\Delta W^{*}=-\frac{1}{2} t * \frac{1}{2} x^{0}=-\frac{1}{4} t * x^{0}$

bzw. 1)

$(V .40) \quad \Delta W^{*}=-\frac{1}{2} T^{*}$.

Dieses Ergebnis gilt auch für mehrere Güter mit linearer Nachfragefunktion, wenn man die Einkommenseffekte der Gütersteuern vernachlässigt ${ }^{2}$. In diesem Falle folgt aus (V.13):

$(v .41)^{3)} \quad \sum_{i} s_{i k} t_{i}=-x_{k}$ mit $k=1, \ldots, n$.

Unter Berücksichtigung der Symmetrie der Substitutionseffekte $s_{i k}=s_{k i}$ entspricht die Mengenänderung von $x_{k}$ aufgrund der Steuererhebung:

(V.42) $\sum s_{k i} t_{i}^{*}=\Delta x_{k}^{*}=-x_{k}^{*}$

und

(v.43) $\quad x_{k}^{\star}-x_{k}^{0}=x_{k}^{*}$

bzw.

1) Vgl. zu diesem Ergebnis Brennan/Buchanan (1977), S. 266

2) Brennan/Buchanan (1980), S. $80 \mathrm{ff}$. leiten das Ergebnis in modifizierter Form für den Zwei-Güter-Fall ab.

3) Das $i$ unter $\sum$ läuft wieder von 1 bis $n$ und wird im folgenden der Einfachheit halber nicht mehr aufgeführt. 
(v.44) $\quad x_{k}^{*}=\frac{1}{2} x_{k}^{0}$ oder $\Delta x_{k}^{*}=-\frac{1}{2} x_{k}^{0}$.

Für das maximale Steueraufkommen gilt:

$(v .45) \quad T^{\star}=\sum t_{i}^{\star} x_{i}^{*}=-\sum t_{i}^{*} \Delta x_{i}^{*}=\sum \frac{1}{2} t^{\star} x_{k}^{0} \cdot$

Die Mehrbelastung hat die Form:

$(V .46) \quad \Delta W^{*}=\sum \frac{1}{2} t_{i}^{*} \Delta x_{i}^{*}$

mit $\Delta x_{i}^{*}=\sum s_{k i} t_{i}^{*}=-\frac{1}{2} x_{k}^{0}$,

so daB wiederum gilt:

(V.47) $\Delta W^{*}=-\frac{1}{2} T^{*} \quad$ q.e.d.

Auf konstitutioneller Ebene könnte die Steuerbemessungsgrundlage dann so lange varijert werden, bis gerade das gewüschte Maximalaufkommen $T^{*}$ erzielt würde. Die Bemessungsgrundlage für $T^{\star}$ kann also unabhängig von der Bemessungsgrundlage einzelner fiuter und von deren Nachfrageelastizitäten fixiert werden.

Dieselben Ergebnisse erhält man auch im Falle einer proportionalen Einkommensteuer mit fixem Freibetrag $\left(x_{E}\right)$. Das maximal realisierbare Steueraufkommen entspricht:

(V.34) $\quad T_{E}^{\star}=\frac{1}{2} t^{\star}\left(x^{0}-x_{E}\right) \cdot$

Die Mehrbelastung beträgt unter Berücksichtigung von (V.30) und (v.32):

( .48$)$

$$
\Delta W_{E}^{\star}=\frac{1}{2} t^{\star}\left(x^{\star}-x_{E}\right)=\frac{1}{4} t *\left(x^{0}-x_{E}\right) \text {, }
$$


so daß wiederum gilt:

(V.49) $\quad \Delta W_{E}=\frac{1}{2} T_{E}^{\star}$.

Bei der Wahl der optimalen Steuerbemessungsgrundlage für $T$ * spielt es also keine Rolle, ob man sich auf eine proportionale oder indirekt progressive Einkommensteuer festlegt, da beide dasselbe Verhältnis von Mehrbelastung zu Steueraufkommen auszeichnet. Allerdings wäre die linear progressive Einkommensteuer mit entsprechendem Freibetrag $X_{E}$ ein sehr flexibles Instrument zur Begrenzung des Steueraufkommens. Bei anderen progressiven Steuertarifen sind die Ergebnisse jedoch keineswegs so eindeutig. Im allgemeinen muß man davon ausgehen, daß progressive Steuern normalerweise eine höhere Mehrbelastung erzeugen als proportionale Steuertarife bei gleichem maximalem Steueraufkommen ${ }^{1)}$. Daher sind proportionale und linear progressive Steuertarife die effizienteren strukturen, wenn $T^{*}$ durch die Reduktion der Steuerbemessungsgrundlage erzielt werden soll. Diese Vorschläge zur Begrenzung der Steuerbemessungsgrundlage stehen in eindeutigem widerspruch zur OT. ATlerdings bleibt bei Brennan/Buchanan offen, welche Teile der Steuerbemessungsgrundlage unter diesen Voraussetzungen von der Besteuerung ausgeschlossen werden sollten.

\section{V.3.2.4. Die Begrenzung einzelner Steuersätze}

Die Festlegung von Steuersätzen unterhalb der Maximalsätze reduziert bei gegebener Steuerbemessungsgrundlage nicht nur das maxi-

1) Vgl. Brennan/Buchanan (1977), S. 267. Für linear ansteigende Grenzsteuersätze gilt beispielsweise: $T^{*}=W^{\star} . V g l$. Brennan/Buchanan (1980), S. $42 \mathrm{ff}$. Das Spektrum progressiver Steuertarife ist allerdings so breit, daß es den Rahmen der Arbeit sprengen würde, sollte man sie einzeln in bezug auf ihr Maximalaufkommen und ihre Mehrbelastung hin untersuchen. Abgesehen davon ist die Wahl des Progressionsgrades nicht nur ein Effizienzproblem. Sie hängt auch auf konstitutioneller Ebene von Gerechtigkeitserwägungen $a b$, so daß der Wahlausgang (auch aufgrund des Arrow-Paradoxons) nicht determiniert ist. Progressive Steuertarife sollen hier auch deshalb ausgeklammert bleiben, weil das vorliegende Leviathanmodell zur Analyse von proportionalen Steuerstrukturen konzipiert wurde. Für die Zwecke dieser Untersuchung reicht es daher aus, auf die Analyse von Brennan/Buchanan (1980) zu verweisen. 
male Steueraufkommen, sondern auch die Mehrbelastung. Aus

$$
\Delta W=\frac{1}{2} \text { t } \Delta x=\frac{1}{2} \tau^{2} \varepsilon q x \text {, mit } \tau=t / q
$$

folgt, daß die Mehrbelastung mit dem Quadrat des Grenzsteuersatzes variiert; d.h. mit sinkendem Steuersatz vermindert sich die Mehrbelastung uberproportional. Im Falle eines Leviathan sind daher auch einzelne Steuersatzbegrenzungen prinzipiell wünschenswert ${ }^{1}$, solange das Steueraufkommen über $T$ * liegt. Auch dieses Ergebnis widerspricht den OT-Empfehlungen. Könnte man jedoch alle Steuersätze bei gegebener Steuerbemessungsgrundlage fixieren, so entspricht die Frage nach der effizientesten Steuersatzstruktur, mit deren Hilfe $T^{*}$ erreicht werden könnte, exakt jener der OT. Weiterhin impliziert ( $V .50)$, daB man $T$ * auch dadurch effizienter realisieren könnte, daß man alle maximalen Steuersätze reduziert und gleichzeitig die Steuerbemessungsgrundlage soweit ausdehnt, bis $T^{*}$ bei niedrigen Steuersätzen wieder erreicht wird. Da in der Ausgangsposition das maximale Steueraufkommen bei maximaler Mehrbelastung erhoben würde $\left(\Delta W=\frac{1}{2} T^{*}\right)$, wird logischerweise jede $\operatorname{Re}-$ duktion von $t^{*}$ bei gleichem $T^{\star} z u$ einer Minderung der Mehrbelastung führen müssen. Die Analyse läßt den Schluß zu, daß geeignete Kombinationen von Steuerbegrenzungsregeln effizientere Ergebnisse liefern können als ihr isolierter Einsatz.

Die Ergebnisse zeigen ferner, daß auch auf konstitutioneller Ebene traditionelle Effizienzerwägungen angestellt werden sollten, da nicht jede Form der Steuerbegrenzung gleichermaßen effizient ist. Die Steuerwirkungsanalyse im allgemeinen Gleichgewichtsmodell muB daher auch Basis konstitutioneller Steuerreformvorschläge sein.

\section{V.3.2.5. Begrenzungen des Steueraufkommens}

Bei der Begrenzung des Steueraufkommens kann man zwischen spezifischen und allgemeinen Begrenzungen unterscheiden ${ }^{2}$ ). Sie können fer-

1) Vgl. Brennan/Buchanan (1980), S. $196 \mathrm{ff}$.

2) Vgl. Brennan/Buchanan (1980), S. $200 \mathrm{ff}$. oder Folkers (1983a), S. $45 \mathrm{ff}$. 
ner in absolute Größen oder bezogen auf eine andere ökonomische Größe, beispielsweise das Sozialprodukt (Anteilsgrenzen) ${ }^{1)}$, und in realen oder nominalen Größen ${ }^{2}$ ) ausgedrückt werden. Der Nachteil solcher Beschränkungen liegt in ihrer Starrheit und in ihren ungewissen allokativen Effekten.

Beispielsweise könnte man sich vorstellen, daß der Anteil einzelner Steuern am Gesamtaufkommen auf konstitutioneller Ebene so fixiert würde, daß er in den Folgeperioden nur mit $t^{\text {max }}$ erreicht werden kann, während das Aufkommen einer anderen Steuer berejts mit einem sehr niedrigen Steuersatz erzielt wird. T* wirde in diesem Fall ineffizienter realisiert als es durch andere Begrenzungsregeln möglich wäre. Diesem Verfahren haftet somit eine gewisse Willkür ${ }^{3}$ an, da auf konstitutioneller Ebene die Anderungen der ökonomischen Daten in den Folgeperioden nicht ausreichend berücksichtigt werden können.

Die Begrenzung des gesamten Steueraufkommens auf $T$ * oder aber die Festschreibung des Verhältnisses von T* zum Sozialprodukt zielt dagegen auf die Einschränkung der gesamten Statstätigkeit ab ${ }^{4}$. Diese Vorschriften würden weitgehend der Begrenzung der Staatsausgaben ${ }^{5)}$ entsprechen, falls zusätzlich der materielle Budgetausgleich gefordert würde. Andernfalls könnten weitere staatsaktivitäten statt durch Steuererhöhungen durch zusätzliche Kredite finanziert werden. Ohne bindende Kreditvorschriften stellt die Ausgabenbegrenzung die wirksamere Restriktion dar 6 ). Eine absolute Begrenzung von $\bar{T}^{\star}$ hat vor allem den Nachteil allzu großer Inflexibilität, da Veränderungen des Sozialprodukts sowie Inflations - und Bevölkerungsentwicklungen nicht berücksichtigt wer-

1) Vgl. Folkers (1983a), S. 44

2) $\mathrm{Vgl}$. ebenda

3) Vgl. zu einer skeptischen Einschätzung dieses Verfahrens Folkers (1983a), S. 45 und Grossekettler (1982), S. 507

4) Vgl. Buchanan (1979), S. 356

5) Vgl. zur Diskussion von Ausgabenbegrenzungen Wildavsky (1980), Folkers (1983a), S. $47 \mathrm{ff}$.

6) Vql. Folkers (1983a), S. 47 
den ${ }^{1)}$. Statt absoluter Steuergrenzen hat man daher die Festlegung von Anteilsgrenzen vorgeschlagen; d.h. das Steueraufkommen wird auf einen bestimmten Prozentsatz des Sozialprodukts festgelegt ${ }^{2}$. Auch wenn dieser Vorschlag eine bessere Anpassung an die Anderung ökonomischer Daten erlaubt, bleiben drei wichtige Fragen offen:

a) Wie kann ein optimaler Staatsanteil bestimmt werden ${ }^{3}$ ),

b) welche Steuerstruktur würde der Leviathan in diesem Falle wählen ${ }^{4)}$ und

c) welche Reaktionen des Leviathan würden durch Steuergrenzen ausgelöst?

ad a) Da die normative Frage der optimalen Statstätigkeit jedoch praktisch kaum bestimmbar ist, reduzieren sich die politisch bedeutsamen Konzepte auf den zweiten Typ der Anteilsbegrenzung, welcher von dem positiven politischen Argument ausgeht, Verminderungen der Statsquote seien nicht durchsetzbar, so daß nur eine Festschreibung der gegebenen Anteilswerte realisiert werden könne ${ }^{5)}$. Ob die These von der oberexpansion des Staatssektors ausreichend belegt ist und daher Steuergrenzen gerechtfertigt werden können, wird von Kritikern dieses Ansatzes heftig bestritten ${ }^{6}$.

ad b) Die Begrenzung des Steueraufkommens kann weder ineffiziente noch ungerechte Steuerstrukturen verhindern. Sollte diese Regelung in Kraft treten, wären ergänzende fiskalische Regeln, die auf die Struktur des Steuersystems einwirken, zwingend erforderlich, um der potentiellen Willkür des Leviathan zu begegnen.

1) Vgl. Folkers (1983a), S. 48

2) Vgl. Buchanan (1979), S. 356 oder Folkers (1983a), S. 49

3) Vgl. Buchanan (1979), S. 357

4) Vgl. Folkers (1983a), S. 51

5) Vgl. Folkers (1983a), S. 50

6) Vgl. Musgrave (1981a). Auf diesen Problembeweis wird in Kapitel VIII noch ausführlich eingegangen. 
ad c) Es ist zu erwarten, daB der Leviathan die Begrenzung des Steueraufkommens nicht passiv hinnehmen wird. Die erwünschte Reaktion wäre die Kostensenkung der staatlichen Leistungserstellung, die aus empirischer Erfahrung nur in sehr geringem Maße eintritt ${ }^{1}$ ).

Eine weitere erfolgversprechende Strategie des Leviathan besteht darin, die öffentlichen Leistungen dort zu kürzen, wo sie den unorganisierten Steuerzahler treffen ${ }^{2}$. Die daraus resultierende Unzufriedenheit der Steuerzahler kann dazu genützt werden, die Steuerbegrenzungen zu lockern oder ganz aufzuheben.

Die Beispiele verdeutlichen, daB isolierte konstitutionelle Steuerregeln kaum Aussicht auf Erfolg haben werden. Einen wirksamen Schutz vor dem Leviathan könnte daher nur eine Steuerverfassung mit mehreren sich ergänzenden Regeln bieten. In diesem Zusammenhang sollten jedoch nicht nur ergebnisbezogene Begrenzungsregeln Berücksichtigung finden. Wie die folgenden oberlegungen zeigen, können verfahrensmäßige fiskalische Beschränkungen einerseits eine flexiblere Anpassung von $T^{*}$ an sich ändernde ökonomische Entwicklungen in den postkonstitutionellen Perioden gewährleisten und andererseits das Ausbeutungspotential des Leviathan weiter reduzieren.

\section{V.3.2.6. Zweckgebundene Steuern}

Bisher stand die Begrenzung von $T^{\max }$ bei gegebenem $\bar{\alpha}$ im Vordergrund. Tatsächlich ist a jedoch nicht exogen gegeben, d.h. der Anteil des Steueraufkommens für die Bereitstellung von öffentlichen Gütern ( $\left.\alpha T^{\star}\right)$ kann durch geeignete fiskalische Verfahrensregeln beeinflußt werden. Der Fiskus wird versuchen, $\alpha$ und damit den gemeinnützigen Anteil am Steueraufkommen soweit wie möglich zu senken. Um die Minimierung der Menge öffentlicher

1) Vgl. Folkers (1983a), S. $108 \mathrm{ff}$.

2) In demokratischen Staatswesen sind Leistungskürzungen vor allem bei unorganisierten bzw. politisch unbedeutsamen Gruppen beliebt. Vgl. Folkers (1983a), S. 106 oder Benkert (1983), S. 475 
Güter ( $z$ ) zu verhindern ${ }^{1}$ ), sollte die Steuerbemessungsgrundlage komplementär zu z gewählt werden. Kann sie von den Steuerzahlern frei variiert werden, ist es der Regierung nur dann möglich, das Steueraufkommen zu erhöhen, wenn sie durch zusätzliche Bereitstellung von $z$ einen Anreiz schafft, der die Steuerzahler veranlaBt, die Steuerbemessungsgrundlage auszuweiten, d.h. die zu z komplementären, besteuerbaren Güter vermehrt nachzufragen. Ein optimum wäre dann erreicht, wenn bei vorgegebener Steuerstruktur das gesamte Steueraufkommen für die gewünschten öffentlichen Güter ausgegeben wird ${ }^{2)}$. Eine Schwierigkeit dieses Ansatzes liegt ähnlich wie beim Aquivalenzprinzip darin, daB es keine Komplementarität zwischen bestimmten öffentlichen Gütern und einer denkbaren Steuerbemessungsgrundlage gibt, oder daB diese auch im Maximum zu gering ist, um das benötigte Steueraufkommen und damit $z$ zu realisieren. Die Einengung des politischen Spielraums dagegen ist in einem Leviathanstaat nicht nur kein Nachteil, sondern das Ziel der Zweckbindung (earmarking) ${ }^{3}$ ) von Steuern ${ }^{4}$ ).

Im Rahmen der vorliegenden Modellkonzeption sind fiskalische Begrenzungen der Steuergewalt unverzichtbare Bestandteile einer Verfassung. Allerdings wird gegenüber der konstitutionellen Theorie der Besteuerung Kritik geübt, die sich teils auf den Ansatz selbst, teils auf die von Brennan/Buchanan gezogenen Schlußfolgerungen bezieht.

1) Vgl. Brennan/Buchanan (1978), S. 306

2) Vgl. Brennan/Buchanan (1978), S. $306 \mathrm{ff}$. Die hinter dem "veil of ignorance" gesellschaftlich gewünschte Menge öffentlicher Güter wird i.d.R. größer sein als die Menge, die der Leviathan aus Eigeninteresse bereitstellen würde. Ein Minimum an z.B. Verteidigung oder Gesetzgebung wird allerdings jeder Staat bereitstellen. Vgl. auch Brennan/Buchanan (1978), S. 314. Das Niveau des öffentlichen Güterbiundels wird jedoch auch in der konstitutionellen Situation nicht immer eindeutig bestimmbar sein, da dies auch von der Risikoeinstellung der Individuen abhängt. Vgl. dazu die Kritik in V.2.4.

3) Vgl. Brennan/Buchanan (1978), S. 317

4) Ganz anders verhält es sich im Falle wohlwollender, allwissender Planer. $\mathrm{Vgl}$. Brennan/Buchanan (1979), S. 11: "In this institutional setting [benevolent dictator (der Autor)] any constraints such as tax limitation (or electoral processes!) can only be viewed as undesirable restrictions, ranging from mildly irritating to wildly perverse." oder Buchanan (1979), S. 350 . 


\section{V.4. Zur Kritik an den konstitutionellen Steuerregeln}

Die once-and-forever-Fixierung der Steuerverfassung ist eine theoretische Fiktion, die wegen mangelnder Information zum Zeitpunkt ihrer Entstehung keine unbegrenzte Gültigkeit haben kann ${ }^{1)}$. Manche Autoren lehnen gar die gesamte Fiktion des "social contract" 2 ) ab, weil das gesellschaftliche Leben ein kontinuierliches spiel sei und aus keiner natürlichen Ausgangsposition (Buchanan (1976)) mit Hilfe eines Gesellschaftsvertrages gestartet werde. "There never was a contract, there is no contract, and there cannot be a contract at the base of social 1 ife" $^{3)}$. Es muß in bestimmten Zeitspannen eine Reform der Steuerverfassung durchgeführt werden, allerdings ohne daß ihr quasi-permanenter Standard berührt wird. Bei diesen Revisionen muß damit gerechnet werden, daß die Wahl der neuen Regeln ebensovon Selbstinteressen geleitetwirdwie Entscheidungen im Steueralltag. Die unsicheren Resultate aus der Regelsetzung werden miteinander verglichen. Wie bei langfristigen Kapitalinvestitionen werden die vielversprechendsten Regeln gewählt. "Self-interest is, in this case, an infinite regress, self-interest applied to the rules to determine the rules, and so on" 4 ).

Ein weiterer Kritikpunkt knüpft an der Tatsache an, daB moderne Regierungen durcheinen hohen Personalaufwand gekennzeichnet sind. Ohne Zutrittsschranken für den Staatsdienst bestehe eine groBe Wahrscheinlichkeit, daß ein Individuum in der postkonstitutionellen Phase der Leviathanklasse angehöre ${ }^{5)}$. Neben anderen Faktoren hängen seine konstitutionellen Entscheidungen aber auch von seiner Risikoeinstellung ab. Die Mitglieder der Verfassungsversammlung werden ihre Chance, später zur Leviathanklasse zu gehören, somit unterschiedlich einschätzen, weshalb eine verfassungsmäeige Obereinkunft aller Individuen nicht mehr gewährleistet ist. Nach Brennan/
1) Vgl. dazu Goetz (1980), S. 63
2) Die Idee des social contract aus dem Zeitalter der Aufklärung ist die Quelle des Buchanan-Ansatzes. Vgl. Bund (1984), S. $14 \mathrm{ff}$.
3) Gordon (1976), S. 588
4) Goetz (1980), S. 65
5) Val. hierzu West/Corke (1980), S. 395 
Buchanan ist der Grundkonsens jedoch Voraussetzung für eine Steuerverfassung. Geht man weiterhin davon aus, daB der Leviathan einen relativ großen Anteil an der Gesamtbevölkerung beschäftigt, müßte eine Verfassungsregel fordern, daß die Staatsbediensteten den gleichen Steuerregeln unterworfen werden wie alle anderen Bürger. Das Prinzip horizontaler Gerechtigkeit nach dem Nutzenkonzept ${ }^{1)}$ verlangt, daB Individuen, die vor der Besteuerung das gleiche Nutzenniveau realisieren, durch die Besteuerung dieselbe NutzeneinbuBe erleiden sollen. Dies impliziert, daB die Steuerbemessungsgrundlage, im Gegensatz zur Brennan/BuchananThese, alle nicht-monetären Nutzen einschlieBlich der Bequemlichkeit eines Amtes ${ }^{2}$ ) umfaBt.

Da diesen oberlegungen technische Schwierigkeiten entgegenstehen und andererseits die unproduktiven Rentengewinne ${ }^{3)}$ durch die Monopolstellung des Staates entstehen, scheint es konzeptionell vorteilhafter zu sein, wenn auf der konstitutionellen Ebene nichtfiskalische Rahmenbedingungen geschaffen werden ${ }^{4}$ ), die das Leviathanverhalten erst garnicht entstehen lassen ${ }^{5)}$. Denn gerade hinter dem veil of ignorance bietet sich die Chance, einen optimalen institutionellen Rahmen zu schaffen ${ }^{6}$ ). Der Leviathan dagegen ist eine denkbar schlechte politische Institution 7). Antizipierende Individuen wür-

1) Vgl. Feldstein (1976a), Musgrave (1976)

2) Vgl. West/Corke (1980), S. 400. Umfassende Steuerbemessungsgrundlagen sind nicht nur aus Effizienzgründen wünschenswert, sondern können auch als Gerechtigkeitsnormen interpretiert werden. Vgl. dazu Hettich (1979), der Simons' "comprehensive tax base" als Gerechtigkeitsregel interpretiert. Vgl. Simons (1938)

3) Vgl. zu diesem Problem Z.B. Buchanan (1980b), Krueger (1974), oder Buchanan/Tollison/Tullock (1980)

4) Die beinahe ausschließliche Fixierung von Brennan/Buchanan auf fiskalische konstitutionelle Schranken und die Behauptung, daß diese den Leviathan ernsthaft beschränken könnten, erscheint wenig begründet und eher auf Glaubenssätzen zu beruhen: "But our whole construction is based on the belief, or faith, that constitutions can work, and that tax rules imposed within a constitution will prevail." Brennan/Buchanan (1980), S. 10

5) Einige nichtfiskalische Möglichkeiten zur Entmonopolisierung der Bürokratie finden sich bei West/Corke (1980), S. $400 \mathrm{ff}$.

6) Vgl. West/Corke (1980), S. 400

7) Vgl. Brennan/Buchanan (1980), S. 186 
den deshalb ihr Augenmerk primär auf die Funktionsfähigkeit von politischen Institutionen richten und versuchen, mögliche negative Ausprägungen der staatlichen Organisation durch verfassungsrechtlich verankerte, politische Verfahren einzudämmen.

Dazu gehört beispielsweise auch die Stärkung des Parteienwettbewerbes in der Demokratie, damit die Wählerpräferenzen möglichst unverfälscht durch die gewählten Entscheidungsträger berücksichtigt werden ${ }^{1)}$. Je besser es gelingt, die Regierung im Sinne der Wählerwünsche zu beeinflussen, um so weniger restringierend muissen die konstitutionellen Steuerregeln sein. Hier wird deutlich, daB die Konsensfähigkeit und die Eindeutigkeit der Steuerregeln von Brennan/Buchanan auf der Vorhersehbarkeit des politischen Versagens bzw. auf der Unzweifelhaftigkeit der Steuermaximierungshypothese beruhen. Unterstellt man dagegen andere Zielfunktionen des Fiskus oder kann ihn über politisch-institutionelle Regeln gar zu wohlwollendem Verhalten zwingen, resultieren natürlich völlig andere steuerpolitische Ratschläge. Dies ist eine Folge der Eigenschaften konstitutioneller Steuerregeln, die einerseits leitend, aber gleichzeitig beschränkend wirken können. Für einen wohlwollenden Alleinherrscher wirken sie regelmäßig einschränkend und verhindern ein First-Best-optimum; im Falle des Leviathan sind sie Leitlinien der Poltik und beschränken gleichzeitig seine Möglichkeiten, immer mehr Steuern zu erheben.

Die konstitutionelle Wahl stellt also eine Entscheidung über Institutionen dar. Im Gegensatz dazu wird in der OT ausschlieBlich die staatliche Zielfunktion maximiert, ohne zu fragen, ob der Fiskus überhaupt im gesellschaftlichen Interesse agiert. Die beiden Theoriegebäude weichen somit nicht nur in ihren steuerpolitischen Empfehlungen voneinander ab; sie unterscheiden sich vor allem auch in ihren weltanschaulichen Grundlagen.

1) Diese Möglichkeit wird auch von Brennan/Buchanan eingeräumt: "With some reform of this latter type [constitutional changes in the procedures or rules through which political decisions are made (der Autor)] resort to direct fiscal constraints may be neither necessary nor desirable." Brennan/Buchanan (1980), S. 154 
V.5. Die weltanschauliche Basis der Steuerreformtheorien

Die gegenwärtige Kontroverse in der Steuerreformdebatte zwischen Vertretern der OT und einer institutionellen Steuertheorie resultiert auf den ersten BIick aus unterschiedlichen Erwartungen über gegenwärtige und zukünftige Aktivitäten des Staates bzw. aus ihren Vorstellungen über die Wirkungsweise und Wirksamkeit der demokratisch politischen Prozesse. Sie unterscheiden sich ferner durch die Kriterien, die einer Steuerreform zugrundeliegen.

V.5.1. Ein Vergleich der normativen Grundlagen der Steuerreformtheorien

Die normative Basis der OT wird üblicherweise durch den Utilitarisrismus im weiteren Sinne repräsentiert ${ }^{1}$ ), dessen Ziel es ist, die Summe der Nutzen aller Gesellschaftsmitglieder zumaximieren $^{2)}$. Wer die "ungerechte" Besteuerungsnorm bzw. SWF verbindlich festlegen soll, bleibt abgesehen von den bereits erörterten theoretischen Mängeln eine ungelöste Frage der Wohlfahrtsökonomie. Ober läßt mandie Wahl der gesellschaftlichen Zielfunktionen der Regierung, bleibt die Gefahr eines Leviathan bestehen. Nur eine einstimmig verabschiedete Wohlfahrtsfunktion würde die Diskriminierung souveräner Individuen verhindern. Wie also sol1 man Normen oder "letzte Werte" erhalten, deren Spezifikation notwendig ist, um Reformvorschläge abzuleiten? Ersthinter dem Schleier der Umwissenheit ist es möglich, einen Konsens über bestimmte Normen zu finden. Allerdings muB man dann auch die Resultate, die aus diesen Normen folgen, akzeptieren ${ }^{3)}$. Die Modifikation einer Norm ist erst dann möglich, wenn man wegen neuer Informationen auf der gleichen Ebene wiederum einen neuen Kontrakt einstimmig beschließen würde.

1) Vgl. Gleichung (IV.2), S. 64 und Abb. 2, S. 66

2) Vgl. z.B. Stiglitz/Boskin (1977), S. $295 \mathrm{ff}$.

3) Vgl. z.B. Hayek (1981), S. $31 \mathrm{ff}$. Um eine Norm zu akzeptieren, sollten natürlich potentielle Ergebnisstrukturen ohne Bezug auf konkrete individuelle Situationen abschätzbar sein. Hierin liegt mit Einschränkungen eine wichtige Aufgabe der OT. Vgl. in diesem Zusammenhang Kap. IV Abs. 3 und Kap. VII dieser Arbeit. 
Brennan/Buchanan setzen an diesem Punkt an und schlagen die Einstimmigkeitsregel als Norm für eine gute Steuerverfassung vor. Eine derart konzipierte Steuerverfassung solle das Ziel aktueller Steuerreformbestrebungen sein. Nur sie bietet Sicherheit vor einer möglichen Ausbeutung durch die staatliche Steuergewalt. Allerdings lassen sich die Ergebnisse von Brennan/Buchanan, wenn überhaupt, nur dann ableiten, wenn man künftig einen Leviathan vermutet. Unterstellt man wohlwollende Regierungen, so wäre eine Einigung, z.B. auf das Rawls-Kriterium ${ }^{1)}$, unter bestimmten $\mathrm{Be}-$ dingungen denkbar. Einstimmigkeit und OT-Normen müssen sich daher nicht prinzipiell widersprechen. Die Frage, welche Bedingungen auf Verfassungsebene erfüllt sein müßten, damit ideale konstitutionelle Steuerregeln geschaffen werden könnten, muB als unbeantwortet gelten ${ }^{2)}$.

V.5.2. Die Beurteilung des politischen Prozesses in der Steuerreformdiskussion

Die Befürworter des OT-Ansatzes klammern Fragen der Funktionsfähigkeit demokratischer Prozesse aus und unterstellen zumindest implizit, daB die Regeln der OT jederzeit durchsetzbar seien. GemäB dieser Unterstellung wäre es lediglich notwendig, den Stand der Wirtschaft in jeder Periode festzustellen und dann mit Hilfe irgendwelcher politisch akzeptierter bzw. vorgegebener Normen (Effizienz- und Distributionskriterien) die optimalen Steuerregeln zu entwerfen. Eine Reform dieser Art muB daher von Periode zu Periode stattfinden und ist von ihrer Natur her nicht langfristig angelegt. Im Gegenteil, jede langfristige Fixierung der Regeln würde keinen optimalen Zuschnitt auf sich verändernde ökonomische Situationen zulassen. Eine Steuerreform, die eine Steuerverfassung zum Ziel hat, wäre suboptimal und daher unerwünscht.

Brennan/Buchanan bemängeln an der "orthodoxen" OT-Analyse, daB sie

1) Vgl. Rawls (1971) und Brennan/Buchanan (1980), S. XII

2) $\mathrm{Vgl}$. zu einem Aspekt dieser Problematik, nämlich zur Frage, wie eine ideale verfassunggebende Versammlung beschaffen sein sollte Hayek (1981), S. $154 \mathrm{ff}$. 
weder ein Verständnis der beobachtbaren politisch-fiskalischen Prozesse bietet, noch dazu in der Lage sei, Reformen vorzuschlagen, die dem Staatsbürger verständlich sind und von ihm akzeptiert werden ${ }^{1)}$. Ferner unterstellen sie, daB weder der politische ProzeB im Sinne der Wählerpräferenzen funktioniere, noch daB eine periodische Adjustierung der Steuergesetzgebung gemäB den sich ändernden ökonomischen Daten möglich sei. Wenn aber in einer Demokratie keine optimalen Steuerregeln ableitbar sind, und wenndie Regierung, selbst imfalle ihrer Existenz, nicht danach handeln würde, d.h. wenn sie sich nicht an den Präferenzender Wähler orientiert, dann können Steuerreformabsichten, die ähnliche Strukturen aufweisen wie sie inder OT vorgeschlagen werden, eine maximale Ausbeutung der Steuerzahler ermöglichen. Derartige Gefahren sollen durch langfristige, konstitutionelle Regelungen vermindert werden.

Die vorangegangenen Oberlegungen machen deutlich, daß rationale Steuerreformen einerseits von den gewählten Kriterien und andererseits von den gegebenen politischen Institutionen und Entscheidungsmechanismen abhängen. Welchen steuerpolitischen Empfehlungen gefolgt werden kann, hängt somit davon ab, ob die Thesen vom wohlwollenden oder ausbeutenden Diktator die aktuellen politischen Gegebenheiten in adäquater Weise widerspiegeln.

Da Politikern - wie allen anderen Individuen - nutzenmaximierendes Verhalten unterstellt werden muB, scheidet die OT-These vom wohlwollenden Planer als Basis für eine positive steuertheorie aus. Der folgende Vergleich zwischen Leviathan-Regeln und aktuellen Steuerstrukturen soll einen ersten AufschluB darüber geben, inwieweit der Leviathan die geeignetere Arbeitshypothese darstellt.

\section{V.6. Aktuelle und optimierte Steuersysteme}

Ein gewichtiges Argument gegen die Leviathan-Hypothese (im übrigen auch gegen die These vomwohlwollenden Planer) rührt aus den $A b-$ weichungen der aktuellen Steuergesetzgebung gegenüber jener Steuerpolitik, die ein Leviathan-Staat verfolgen wïrde. Es sei noch ein-

1) Vgl. Brennan/Buchanan (1980), S. XII 
mal daran erinnert, daß seine Steuerpolitik darauf ausgerichtet wäre, eine möglichst umfassende steuerbemessungsgrundlage und ein optimal differenziertes Steuersystem zu integrieren. Diese Merkmale optimierter Steuersysteme sind im aktuellen Steuersystem nicht oder nur fragmentarisch zu erkennen ${ }^{1)}$. Aus dem Vergleich zwischen realer Steuerstruktur und zugegebenermaßen idealisierten Leviathanregeln läßt sich daher schließen, daß die Maximierungsergebnisse, werden sie überhaupt angestrebt, politisch nicht durchsetzbar sind ${ }^{2}$ ).

Die Behauptung läßt sich am Beispiel der Verbrauch-und Einkommensteuern belegen. Nach den Leviathan- (und OT-) Regeln wären eine möglichst umfassende Steuerbemessungsgrundlage und ein differenziertes Verbrauchsteuersystem optimal. Tatsächlich aber existiert z.B. in der Bundesrepublik nur ein rudimentär differenziertes Verbrauchsteuersystem mit interpersonell gleichen Steuersätzen, dessen Aufkommenspotential allein schon aufgrund der herabgesetzten Steuersätze für Grundnahrungsmittel noch nicht erschöpft ist ${ }^{3}$ ). Ferner entspricht die Steuerbemessungsgrundlage der geqenwärtigen Einkommensteuer keinesfalls den Wünschen eines Leviathan, weil sie durch eine Vielzahl von Steuerbefreiungen, Steuervergünsti-

1) "Das Steuerrecht der Gegenwart ist nicht nur kein werk aus einem theoretischen Guß, es läßt auch seine ethischen Grundlagen nicht (mehr) erkennen". Tipke (1976). S. 294

2) "Was vor allem deprimiert, ist, daß wie in der Vergangenheit, so auch gegenwärtig wieder (oder noch) ohne jedes Konzept gearbeitet wird ...". "Wieder und wieder haben bei uns wie im Ausland Wissenschaftler aber auch manche Praktiker darüber geklagt, daß wir kein Steuersystem haben, das diesen Namen verdient, das heißt an gewissen obersten Prinzipien und Zielen ausgerichtet ist". Neumark (1983), S. 74. Vgl. zu ähnlicher Kritik auch Littmann (1984), insbesondere S. 73.

3) Auch die Entwicklung der Steuerquoten (ohne Sozialabgaben) weist keine eindeutig steigende Tendenz auf. In der Steuerquote allein kommt die staatliche Dispositionsgewalt jedoch nicht eindeutig zum Ausdruck. Vielmehr sollten auch alle steuerähnlichen Abgaben mitberücksichtigt werden (z.B.Sozialversicherung). Eine so ermittelte Zwangsabgabenquote istallerdings in den letzten Jahren deutlich gestiegen. Val. Z.B. Caesar (1980), S. 13 und 14, Obersichten $2 a$ und $2 b$. Die maximale Äbgabenquote ist aber nach Meinung der meisten Okonomen bisher nur in Ausnahmefällen (z.B. Schweden, vgl. Stuart (1981)) erreicht. Val. zu empirischen Ergebnissen insbesondere Fullerton (1982) und zur Diskussion z.B. Barro (1984), S. 361, Neumark (1981), S. 384 oder Folkers (1983b), S. 203 ff. Trotzdem kann auch nach Meinung vieler Kritiker der sogenannten "Laffer-curve" die Steuerbelastung gemessem am Sozialprodukt bereits zu hoch sein. Vgl. z.B. Neumark (1983), S. 77 oder Fullerton (1982), S. 20 
gungen oder sonstigen Sonderregelungen gekürzt ist ${ }^{1}$. Auch die augenblickliche Diskussion um den Wildwuchs von Subventionen ist ein augenscheinlicher Beweis für eine effizienz- und verteilungspolitisch zielinadäquate Steuerpolitik. Wie bereits in Kapitel III erläutert wurde, stellen bei der Realisierung optimaler Steuerstrukturen vor allem die Informationsdefizite ein erhebliches Hindernis dar. Das gilt ingleichem Maße für den Leviathan. Inwieweit steuerpolitische Entscheidungen bei Unsicherheit fürdie systematische Diskrepanz zwischen aktuellen und optimierten Steuersystemen verantwortlich sind, soll im folgenden analysiert werden.

\section{V.7. Zusammenfassung}

Die vorliegende Untersuchung demonstriert, daß die Fehlspezifikation statlichen Verhaltens zu steuerpolitischen Empfehlungen führen kann, die keineswegs im Interesse der Steuerzahler liegen. Optimale Effizienzregeln der OT können in den Händen eines Leviathanstaates zum perfekten Ausbeutungsinstrument werden. Aus dieser Perspektive muß die Vernachlässigung suboptimaler (politischer) Steuerinstitutionen, wie dies im OT-Ansatz der Fall ist, als schwerwiegender Nachteil gewertet werden. Denn steuerpolitische Entscheidungsträger, die einen nicht unbeträchtlichen spielraum zur Durchsetzung eigener Interessen haben und daher auch nicht gezwungen sind, Wählerpräferenzen durch adäquates politisches Handełn zu verfolgen, gehören zur Realität politischer systeme.

Sieht man von der Diskrepanz zwischen Leviathan- und OT-Steueraufkommen einmal ab, so erscheint es besonders problematisch, daß sich die Strukturen beider Steuersysteme gleichen und somit die Kontrolle des Fiskus außerordentlich schwierig wird. Deshalb stellt sich die Frage, ob institutionelle Störungen, die dem politischen system inhärent und oftmals unabänderbar sind, ein Abweichen von OT-Empfehlungen ratsam erscheinen lassen, um poten-

1) Vgl. Folkers (1983b), S. 203 
tieller Ausbeutung zu entgehen und damit eine gesellschaftiche Wohlfahrtssteigerung zu erreichen. Demnach gilt es, eine möglichst weitgehende Kongruenz zwischen politischem Handeln und Wählerwillen zu erzeugen. Zu diesem Zweck stehen quantitative und verfahrensmäßige Regelungen zur Verfüqung. Es muß jedoch damit gerechnet werden, $d a \beta$ die institutionalisierte Regelbindung des Fiskus, gemessen an den Ergebnissen der OT, normalerweise zu suboptimalen Ergebnissen führt. Die Wohlfahrtsdifferenz zwischen beiden Ergebnissen kann somit als Ausmaß der Kontrollkosten staatlicher Steuermacht interpretiert werden. Der effektive gesamte Wohlfahrtsgewinn der Steuerverfassung dürte angesichts eines Leviathan jedoch beträchtlich sein.

Um institutionelle Regeln dem Zugriff eigensüchtiger Regierungen zu entziehen, müssen sie Verfassungsrang erhalten, so daß sie nur noch von qualifizierten Mehrheiten modifiziert werden können. Welche konkreten Steuerregeln gewählt werden, hängt von den Erwartungen über den laufenden politischen Prozeß ab. Beispielsweise sind für die Brennan/Buchanan-Regeln ganz speziell die Leviathanthese und die Tatsache verantwortlich, daß es in diesem Modell nur ökonomische und keinerlei politische Restriktionen gibt. Werden andere Institutionen und neben den ökonomischen auch politische Restriktionen unterstellt, so ändern sich die konstitutionellen Regelempfehlungen. Auch die postulierte Einstimmigkeit bei Brennan/Buchanan würde dann nicht mehr gesichert sein. Gerade aus diesem Grunde bedarf es einer Oberprüfung der politisch-ökonomischen Relevanz des Leviathanmodells, das durch Pessimismus und tiefes Mißtrauen gegenüber staatlichen Eingriffen geprägt ist. Ein Vergleich zwischen den aktuellen und Optima 1-Steuersystemen belegt, daß das Leviathanmodell nur bedingt als Spiegelbild der Realität akzeptiert werden kann. Inwieweit sich diese Abweichung von der Realität durch die Annahme unvollkommener Informationen über das Nachfrageverhalten der Konsumenten erklären läßt, ist Gegenstand des folgenden Kapitels. 
VI.1. Problemstellung

In den bisherigen Modellen der optimalen Steuerplanung wurde vollkommene Information unterstellt. Die Realitätsferne dieser Annahme ist offenkundig. Sie ist daher als Grundlage finanzpolitischer Empfehlungen ungeeignet; denn die Individuen einer Gesellschaft treffen ihre ökonomischen und politischen Entscheidungen bei mehr oder minder großer Unsicherheit. Informationsiücken bei der Datenerfassung und -verarbeitung sind somit auch Charakteristika staatlicher Steuerplanung und von großer Bedeutung für die steuerpolitische Praxis').

Im letzten Kapitel wurde angedeutet, daß viele Steuerreformentscheidungen nur aufgrund erheblicher Informationsmängel zu erklären sind. Das folgende Modell erleichtert die Isolation der Risikokomponente steuertheoretischer optimierungskalküle, indem es einen relativ einfachen Vergleich mit dem Leviathanmodell in Kap. $V$ zuläßt. Dabei wird gezeigt, daß die Abweichungen zwischen den Leviathanregeln bei Sicherheit und Unsicherheit über das künftiage Nachfrageverhalten der Konsumenten entscheidend von der Risikoeinstellung der Entscheidungsträger abhänqen. Da OT- und Leviathanmodelle gleichgelagerte Entscheidungsprobleme aufweisen, ist es in diesem Zusammenhang völlig ausreichend, nur einen der beiden Modelltypen exemplarisch zu untersuchen. Außerdem wurde in Kap. II und $V$ gezeigt, daßihre optimierungskalküle auf derselben Informationsbasis, d.h. auf denselben ökonomischen Plandaten basieren.

\section{VI.2. Das Modell}

Abgesehen von der Einführung der Entscheidungskriterien bei Risiko bleibt die Grundstruktur des Ein-Konsumenten-Modells bei vollkomme-

1) Vgl. Z.B. Bartlett (1973): " ... first government cannot know for certain what they should do, and secondly they cannot know for certain what they are doing". 
ner Information erhalten. Die sich aus den risikobehafteten optimierungskalkiulen ergebenden Modifikationen werden im folgenden erläutert.

\section{VI.2.1. Das Entscheidungskriterium}

Ein wichtiges Entscheidungskriterium bei Risiko ist das Erwartungswertkriterium (auch $\mu$-Kriterium ${ }^{1}$ ) genannt). Nach diesem Kriterium ist diejenige Alternative optimal, die den Erwartungswert des Steueraufkommens maximiert. Können einzelnen Steuerstrukturen Wahrscheinlichkeitswerte zugeordnet werden, so erhält mandie folgende Steueraufkommensmatrix ${ }^{2}$ ), wobei die Symbole folgende Bedeutung haben: $A_{d}$ sind die alternativen Steuersysteme, $S_{e}$ diemöglichen Zustände der Gesellschaft, $W_{e}$ die diesen Zuständen zugeordneten Wahrscheinlichkeiten und $T_{\text {de }}$ die Steueraufkommen, die zu erwarten sind, wenn beim Steuersystem d die Umweltkonstellation e existiert.

Steueraufkommensmatrix ${ }^{3)}$ :

\begin{tabular}{|c|c|}
\hline$w_{e}$ & $w_{1} \ldots w_{e} \ldots w_{h}$ \\
\hline $\mathrm{S}_{\mathrm{e}}$ & $s_{1} \ldots s_{e} \ldots s_{h}$ \\
\hline$A_{1}$ & $T_{11} \cdots T_{1 e} \cdots T_{1 h}$ \\
\hline . & • \\
\hline - & • \\
\hline • & • \\
\hline$\cdot$ & $\cdot$ \\
\hline$A_{d}$ & $T_{d 1} \cdots T_{d e} \cdots T_{d h}$ \\
\hline
\end{tabular}

Die Steueraufkommensmatrix enthält die Niveaus der Steueraufkommen, die aus verschiedenen Steuersystemen bei alternativen Gesellschaftszuständen resultieren.

1) Vgl. dazu z.B. Schneeweiß (1967), S. 52 ff. oder Bitz (1981), S. 90 ff.

2) Die Steueraufkommensmatrix entspricht der Ergebnismatrix in der Entscheidungstheorie. Vgl. zur Ergebnismatrix Z.B. Pfohl/Braun (1981), S. 39

3) Die Steueraufkommensmatrix geht auf die Ergebnismatrix bei v. Neumann/ Morgenstern (1974) zurück. 
Da die Finanzexperten in unserem Beispiel die Eintrittswahrscheinlichkeit der $S_{e}$ kennen, handelt es sich um Entscheidungen unter Risikol). Der mathematische Erwartungswert $\mu$ lautet:

(VI.1) $\mu_{\mathrm{d}}=\sum_{\mathrm{e}} T_{\mathrm{de}} W_{\mathrm{e}}$

Um Problemen des $\mu-K r i t e r i u m s$ aus dem Wege zugehen ${ }^{2}$ ), und um die Risikoeinstellung von Entscheidungsträgern berücksichtigen zukönnen, wird zusätzlich die Standardabweichung in das Entscheidunaskriterium integriert. Die Standardabweichung isthier ein MaB für die Streuung der potentielien Steueraufkommen bei qegebenem Steuersystem und alternativen Reaktionender Steuerzahler; sie dient als Risikoma für die fiskalischen Entscheidungsträger. Die Risikoeignung des Leviathan hängt davon $a b$, welche Standardabweichung er bei beliebigen Alternativen mit demselben Erwartungswert präferiert. Kennt man die Entscheidungskriterien, so 1 äßt sich die Präferenzfunktion des Fiskus in Abhängigkeit von u und $\sigma$ als

$$
(V I .2) \quad u=u(\mu, \sigma)
$$

schreiben, wobei $\partial u / \mu \sigma$ den Grenznutzen des Risikos darstellt und

(VI.3) $\quad \frac{\partial u}{\partial \sigma}<0$ Risikoscheu,

(VI.4) $\quad \frac{\partial u}{\partial \sigma}=0 \quad$ Risikoneutralität und

(VI.5) $\quad \frac{\partial u}{\partial \sigma}>0$ Risikofreude

implizieren. Sofern die Standardabwichung relativ klein wird, kann das $\mu-\partial-P r i n z i p$ als Approximation beliebiger Nutzenfunktionen nach dem Bernoulliprinzip dienen ${ }^{3}$. Obwohl (VI.2) a priorinicht

1) Vgl. dazu Z.B. Salinger (1981), S. 42

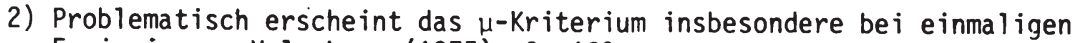
Ereignissen. Vgl. Laux (1975), S. 160

3) Vgl. Sinn (1980). S. $126 \mathrm{f}$. In etwas anderem Zusammenhang Samuelson (1970), S. 537-542 und Tsiang (1972) 
als repräsentative Präferenzstruktur staatlicher Entscheidungsträger interpretiert werden kann, stellt sie eine mögliche Zielfunktion dar. Das $\mu-\sigma-K r i t e r i u m ~ f i n d e t$ deshalb Eingang in diese Analyse, weil es ein potentielles Verhalten politischer Akteure charakterisiert und in anschaulicher Weise die Leviathan-Ergebnisse modifiziert ${ }^{1}$ ). Die Kriterien der Risikoneigung der staat1ichen Entscheidungsträger [Gleichungen (VI.3) - (VI.5)] sind allerdings dadurch gekennzeichnet, daB ihre Werte durch eine monotone Transformation der Präferenzfunktion beeinfluBt werden. Diesen Nachteil kann man durch ein alternatives MaB der Risikoneigung vermeiden, das aus dem totalen Differential der Zielfunktion $u(\mu, \sigma)$ gewonnen wird $\left.{ }^{2}\right)$ :

(VI.6) $\quad \frac{\partial u}{\partial \mu} d \mu+\frac{\partial u}{\partial \sigma} d \sigma=0$.

(VI.6) entspricht somit einer Bewegung entlang einer Indifferenzkurve. Das MaB der Risikoneigung wird durch die Grenzrate der Substitution zwischen dem mathematischen Erwartungswert und der Varianz der Zielgröße (z.B. Gewinn) definiert. Aus (VI.6) folgt:

(VI.7) $\quad \frac{d \mu}{d \sigma}=-\frac{\partial u / \partial \sigma}{\partial u / \partial \mu}=R$.

Das Risikoma $B$ des staatlichen Entscheidungsträgers gibt daher an, wie sich sein Nutzenniveau in Abhängigkeit von der Variation der Standardabweichung der Steuereinnahmen, gewichtet mit einer marginalen Zunahme des Erwartungswertes der Steuereinnahmen, ändert. Anders ausgedrückt entspricht $R$ dem "marginal reward for risk taking" ${ }^{3)}$. Unter der Voraussetzung $\partial u / \partial \mu>0$ und $\partial u / \partial \sigma \gtreqless 0 \mathrm{folgt}^{4}$ ):

1) Viele Autoren sind von der Oberlegenheit des $\mu-\sigma-K r i t e r i u m s$, verglichen mit anderen Kriterien, überzeugt. Vg1. Z.B. Magnusson (1969), S. 26 oder Sinn (1980), S. $126 \mathrm{ff}$.

2) Vgl. dazu Magnusson (1969), S. 26 f. Ein Modell mit eben diesem Maß der Risikoneigung findet sich bei Folkers, D. (1975), S. $19 \mathrm{ff}$.

3) Magnusson (1969), S. 27

4) Vgl. Magnusson (1969), S. 66 
(VI.8) R>0 Risikoscheu,

(VI.9) $\quad R=0$ Risikoneutralität,

(VI.10) $\quad R<0$ Risikofreude.

Die Risikopräferenzen hängen somit nach wie vor von der Einschätzung der Standardabweichung ab. Gleichung (VI.7) zeigt beispielsweise, daB die Risikofreude um so mehr wächst, je stärker das Nutzenniveau mit steigendem o zunimmt und jekleiner die positive Nutzenänderung mit steigendem $\mu$ ausfällt.

Gegeben seien $n$ Güter, deren Mengen durch $x_{i}(i=1, \ldots, n)$ symbolisiert werden. Der Einfachheit halber mögen die nachgefragten Mengen lediglich vom eigenen Preis des jeweiligen Gutes abhängen $\left(x_{i}=x_{j}\left(q_{j}\right)\right)$. Die Nachfrage nach diesen Gütern sei eine Zufallsvariable, deren Erwartungswert $\mu_{x_{i}}$ und Standardabweichung $\sigma_{x_{i}}$ folgende Eigenschaften besitzen $\left.{ }^{1}\right)^{i}$ :

(VI.11) $\quad \mu_{x_{i}}=\mu_{x_{i}}\left(a_{i}\right)$, mit $\frac{\partial \mu_{x_{i}}}{\partial q_{i}}<0$ und

(VI.12) $\quad \sigma_{x_{i}}=\sigma_{x_{i}}\left(a_{i}\right)$, mit $\frac{\partial \sigma_{x_{i}}}{\partial q_{i}} \gtreqless 0$.

Gleichung (VI.11) besagt, daB sich der Erwartungswert der Zufallsvariablen $x_{i}$ entsorechend einer fallenden Preis-Absatz-Funktion bei sicheren Erwartungen verhalten wird. Diese Hypothese drückt den normalerweise vorliegenden Sachverhalt aus, daB steigende Preise die nachgefragten Mengen sinken lassen und deshalb im stochastischen Nachfragemodell zu sinkenden Erwartungswerten der Nachfrage führen; umgekehrt verhält es sichbei sinkenden Preisen. Da ähnliche Wirkungsrichtungen von Preisänderungen auf die Standardabwei-

1) Eine ausführliche Diskussion über das Vorzeichen der Verteilungsparameter in einem stochastischen Ein-Produkt-Nachfragemodell findet sich Z.B. bei Folkers, D. (1975), S. $24 \mathrm{ff}$. 
chung nicht auf der Hand liegen, läßt Gleichung (VI.12) zunächst alle Möglichkeiten offen. Aus technischen Gründen muB allerdings angenommen werden, daB (VI.11) und (VI.12) jeweils stetige und zweimal differenzierbare Funktionen sind.

Die Gleichungen (VI.11) und (VI.12) implizieren die ebenfalls nicht unproblematische Annahme, daß die Verteilungsparameter $\mu_{x_{i}}$ und $\sigma_{x_{i}}$ nur vom eigenen Preis abhängen. In der Regel wird dieser Sachverhalt kaum erfüllt sein, da die Nachfrage nach einem Gut üblicherweise auch von anderen Güterpreisen mitbestimmt wird. Um diese Voraussetzung dennoch aufrechtzuerhalten, sei unterstellt, daß der Leviathan das Steueraufkommen lediglich für einen ausgewählten Kreis von Konsumgütern maximieren möchte. Diese Konsumgüter mögen sich durch eine starke Eigenpreisabhängigkeit auszeichnen, während Preisinterdependenzen unbekannt seien. Unter diesen Voraussetzungen stellen dieGleichungen (VI.11) und (VI.12) plausible Funktionalbeziehungen dar. Die Steuerbeträge pro Mengeneinheit ihrerseits sind problemlose Instrumentvariablen des Fiskus, da sie, wie im OT-Modell, die Differenz zwischenden Konsumentenund den fixen Produzentenpreisen darstellen. Die Steuereinnahmen des Staates auf den einzelnen Märkten ( $\left.T_{j} ; i=2, \ldots, n\right)$ sind ebenfalls Zufallsvariable, die sich aus den Steuerbeträgen multipliziert mit Mengeneinheiten ergeben. Daher lassen sich Erwartungswert und Standardabweichung der jeweiligen Steuergewinne in Abhängigkeit von den jeweiligen Marktpreisen schreiben:

(VI.13) $\quad \mu_{T_{i}}\left(q_{i}\right)=t_{i} \mu_{x_{i}}, b z w$.

$\left(\begin{array}{lll}\text { VI.14) } & \sigma_{T}\left(q_{i}\right)=t_{i} & \sigma_{x_{i}}\end{array}\right.$

Das gesamte Steueraufkommen

(VI.15) $\quad T=\sum_{i} t_{i} x_{i}(q)$

ist somit eine von den jeweiligen Steuereinnahmen abhängige Zufalls- 
variable, deren Verteilungsparameter folgende Form haben ${ }^{1)}$ :

(VI.16)

$$
\mu_{T}=\mu_{\left(\sum_{i} T_{i}\right)}=\sum_{i} \mu_{T_{i}}\left(q_{i}\right), b z w .
$$

(VI.17) $\sigma_{T}=\sigma_{\left(\sum_{i} T_{i}\right)}=\sqrt{\sum_{i=1}^{n} \sigma_{T}^{2}\left(q_{i}\right)+2 \sum_{i=2}^{n-1} \sum_{h=i+1}^{n} \operatorname{cov} T_{i} T_{h}}$

bzw.

(VI.17) $\quad \sigma_{T}=\sqrt{\sum \sigma_{T}^{2}\left(q_{i}\right)+\operatorname{cov}_{T}}$.

\section{VI.2.2. Leviathanregeln bei Unsicherheit}

Der Leviathan möchte auch im stochastischen Nachfragemodell das Steueraufkommen maximieren. Allerdings sind nun die Wirkungen seiner Steuerinstrumente auf die Nachfrage und somit auch auf das Steueraufkommen nicht mehr sicher.

In der Zielfunktion der staatlichen Entscheidungsträger finden sich daher die Verteilungsparameter des Steueraufkommens:

$(V I .18) \quad V(q)=.V\left(\mu \underset{i}{\left(\Sigma T_{i}\right)} \underset{i}{\left(\Sigma T_{i}\right)}\right)$

Die Präferenzfunktion erlaubt die Bildung einer Rangordnung alternativer Steuersysteme unter Berücksichtigung der jeweiligen Steuerverlustrisiken.

Die Bedingungen für ein Maximum von (VI.18) Iauten $^{2}$ ):

1) Vgl. Markowitz (1970), S. 94

2) Eine ausführliche Diskussion dieser Bedingungen findet sich für ein ähn1 iches Modell monopolistischer Preispolitik bei stochastischer Nachfrage im Falle einer Ein-Produkt-Unternehmung bei Folkers, D. (1975), S. 32 ff. Die Bedingungen zweiter Ordnung werden als erfuillt vorausgesetzt. 
(VI.19) $\quad \frac{\partial V}{\partial q_{i}}=\frac{\partial V}{\partial \mu\left(\sum_{i} T_{i}\right)} \cdot \frac{\partial \mu\left(\sum_{i} T_{i}\right)}{\partial q_{i}}+\frac{\partial V}{\partial \sigma\left(\sum_{i} T_{i}\right)} \cdot \frac{\left.\partial \sigma_{i} T_{i}\right)}{\partial q_{i}}=0$.

Nach einigen Umformungen ergibt sich ${ }^{1)}$

(VI.20) $\quad \tau_{i}=\frac{t_{i}}{a_{i}}=-\frac{1}{\varepsilon\left(\mu_{\left.x_{i}{ }^{\prime} q_{i}\right)}\right.}+\frac{1}{\varepsilon\left(\mu_{\left.x_{i}{ }^{\prime} q_{i}\right)}\right.} R \frac{1}{\mu_{x_{i}}}$

$$
\text { - }\left[\frac{1}{\sigma_{\left(\sum_{i} T_{i}\right)}}\left(\sigma^{2} x_{i} t_{i}+t_{i}^{2} \sigma_{x_{i}} \frac{\partial \sigma_{x_{i}}}{\partial q_{i}}\right)+\frac{\partial \operatorname{cov} T}{\partial q_{i}}\right] \text {. }
$$

Um (VI.20) mit dem deterministischen Ergebnis in Kap. II vergleichen zu können, wird die Risikokomponente neutralisiert. Die Standardabweichung des maximalen Steueraufkommens spielt dann keine Rolle im Kalkiil des Leviathan $(R=0)$. Gleichung (VI.20) reduziert sich deshalb zu der Optimalbedingung (VI.21).

(VI.21) $\quad \tau_{i}^{\prime}=\frac{t_{i}}{q_{i}}=-\frac{1}{\varepsilon_{\left(\mu_{x_{i}}{ }^{\prime} q_{i}\right)}}$,

die man auch dann erhalten würde, wenn die Nutzenfunktion des Leviathans lediglich vom Erwartungswert des Steueraufkommens abhängig wäre.

Für den Fall, daB der Erwartungswert der stochastischen Nachfrage mit der aktuellen Nachfrage übereinstimmt,

(VI.22) $\quad \mu_{x_{i}}=x_{i}$,

ergibt sich aus (VI.21) dieselbe Lösung für den maximalen Steuer-

1) Vgl. dazu Anhang $F$ 
satz wie im deterministischen Modell (IV.18).

Um die Abweichungen des "Risikosteuersatzes" $\tau_{j}$ (VI.20) vom optimalen ${ }^{1)}$ Steuersatz bei sicherheit bzw. bei Risikoneutralität des Entscheidungsträgers bestimmen zu können, müssen die Vorzeichen der Einflußfaktoren auf der rechten Seite von (VI.20) bekannt sein.

Unter Berücksichtigung von (VI.11) weiB man, $d a B \varepsilon_{\left(\mu_{x_{j}}, q_{j}\right)}$ ein negatives Vorzeichen besitzt. Der Erwartungswert der Nachfrage von $x_{i}$, die Varianz, sowie die Standardabweichungen und der Steuersatz haben definitionsgemäß ein positives Vorzeichen. Näherer Betrachtung beduirfen daher nur noch die Ausdrücke $R, \partial \tau_{x_{i}} / \partial q_{i}$ und $\operatorname{covv}_{T} / \partial q_{i}$. GemäB (VI.8), (VI.9) und (VI.10) drückt R die subjektive Risikoeinstellung des Entscheidungsträgers aus. Das Vorzeichen von $R$ kann nur bestimmt werden, wenn die Risikopräferenz des Finanzpolitikers bekannt ist.

Unterstellt man Politikern und Bürokraten Risikoscheu, und geht man von der noch näher zu erläuternden Annahme aus, daß die eckige Klammer auf der rechten Seite (VI.20) ein positives Vorzeichen besitzt, so kann man (VI.20) folgendermaßen interpretieren:

Da $\varepsilon_{\mu_{x_{i}}}<0, \sigma_{T}>0, \mu_{x_{i}}>0,[]>$.0 und $R>0$ gilt, wird der zweite Ausdruck auf der rechten Seite von (VI.20) kleiner als nu11. Dies wïrde bedeuten, daB ein risikoscheuer Leviathan immer unterhalb des gewinnmaximalen Steuersatzes bei Risikoneutralität bleibt ${ }^{2}$. Umgekehrt verhält es sich im Falle eines risikofreudigen Monopolstates.

Die Aussage gilt nur, wenn das Vorzeichen der eckigen Klammer von (VI.20) positiv ist. Das wiederum hängt

1) Optimal ist für den Leviathan jener Steuersatz $\tau_{j}$, der das maximale Steueraufkommen erbringt.

2) Bemerkenswert ist, daß in die Berechnung des optimalen Steuersatzes die Standardabweichung des gesamten Steueraufkommens bzw. das Steueraufkommensrisiko $\sigma_{T}$ eingeht. Das bedeutet, daß $\tau$, auch im Falle völliger Unabhängigkeit der Märkte, immer unter Berücksichtigung aller anderen Konsumentenpreise bestimmt wird. 
davon ab, wie die Standardabweichung der Nachfrage $\sigma_{x_{i}}$ und die Kovarianz cov $\operatorname{cof}_{T}$ Preisänderungen $\Delta q_{j}$ reagieren. Zumeist dürfte eine Preiserhöhung mit einer höheren Standardabweichung der Nachfrage einhergehen, weil steigende Konsumentenpreise zu einer stärkeren Nachfragestreuung ( $z$. B. in unterschiedlichen Konjunktursituationen) führen können. In diesen Fällen wären die beiden ersten Ausdrücke in der Klammer von (VI.20) eindeutig positiv. Es ist jedoch durchaus denkbar, $d a B \partial \sigma_{x_{i}} / \partial q_{i}<0$ wird, weil durch die Preiserhöhung "die Käufergruppe auf eine relativ preisunelastische und regelmäßig kaufende Stammkundschaft reduziert wird"1). A priori muß daher immer mit $\partial \sigma_{x_{i}} / \partial q_{i} \geqq 0$ gerechnet werden.

Neben diesem Unsicherheitsmoment bleibt auch die Anderung der Kovarianz zwischen den Zufallsvariablen aufgrund von Steuersatzvariationen ungewiB. Obwohl die untersuchten Güter keine Preisoder Produktionsinterdependenzen aufweisen, d.h. die Nachfrage der einzelnen Güter nur von ihrem eigenen Preis abhängt, können doch stochastische Korrelationen zwischen nachgefragten Gütermengen bestehen. Auch hier können a priori keine Reaktionen der Kovarianz ausgeschlossen werden.

Allerdings sprechen wegen der Modellkonzeption selbst einige oberlegungen dafür, die Anderungen der Kovarianz aufgrund von Preisänderungen unberücksichtigt zu lassen. Zur Verdeutlichung dieser Argumentation sei von nur zwei Gütern ausgegangen. Die Kovarianz $\operatorname{cov}_{T}$ wird nun $z u^{2}$ )

$\operatorname{cov}_{T_{1} T_{2}}=2 t_{1} t_{2} \operatorname{cov}_{x_{1} x_{2}}=2 t t_{2} \sigma_{x_{1}} \sigma_{x_{2}} \rho_{x_{1}} x_{2}$

$\rho_{x_{1} x_{2}}$ bezeichnet den Korrelationskoeffizienten (Wertebereich +1 bis -1), der ein standardisiertes Maß dafür ist, "in welchem Ausmaß die beiden Zufallsvariablen gleichartig auf Veränderungen der

1) Folkers, D. (1975), S. 26

2) Vgl. dazu Z.B. Bitz (1981), S. $110 \mathrm{ff}$. 
Uinweltzustände reagieren"1). Dieser Reaktionsmechanismus wird im vorliegenden Modell durch vielfältige Größen auf der Nachfrageseite bestimmt, die durch kleine Preisänderungen kaum beeinflußt werden dürten, so daßmanganzallgemein $\partial \rho_{x_{1} x_{2}} / \partial q_{1}=0$ erwarten kann. Darüber hinaus wurde aber im vorliegenden Modell davon ausgegangen, daß Steuersatzvariationen $\partial t_{1}$ keinen Einfluß auf den Erwartungswert und die Standardabweichung von $x_{2}$ haben, d.h. es galt $\mu_{x_{1}}=$ const. und $\sigma_{x_{1}}=$ const. Unter diesen Bedingungen scheint es sehr unwahrscheinlich, daß sich die Verteilungsfunktion von $x_{2}$ durch die Steuersatzvariation von $x_{1}$ derart geändert haben soll, daß exakt dieselben Verteilungsparameterwerte realisiert werden. Unterstellt man deshalb vorgegebene zweidimensionale Verteilungsfunktionstypen ${ }^{2}$ ) für die Zufallsvariablen $x_{j}, i=1,2$, eine Voraussetzung, die beim vorliegenden zweidimensionalen Entscheidungskriterium plausibel erscheint, so implizieren konstante Parameterwerte $\mu_{x_{1}}$ und $\sigma_{x_{1}}$ eine von der konkreten Ausprägung $x_{2}\left(q_{2}\right)$ unabhängige spezifische Verteilungsfunktion. In diesen Fällen wären die Zufallsvariablen $x_{1}$ und $x_{2}$ stets unkorreliert bzw. unabhängig ${ }^{3}$, weswegen ihre Kovarianz verschwindet. Dieser Sachverhalt liegt insbesondere bei Zufallsvariablen vor, die normalverteilt sind ${ }^{4}$. Unter Berücksichtigung dieser Oberlegungen läBt sich aus (VI.20) für einen risikoscheuen Leviathan folgende Aussage ableiten: Der optimale Steuersatz $\tau_{i}$ wird unterhalb von $\tau_{i}^{i}$ liegen, wenn der Leviathan vermutet, daB er durch diese Steuersatzsenkung sein Gesamtrisiko verringern kann. Gemäß seiner Präferenzfunktion gewichtet er diese Risikosenkung höher als die Abweichung vom maximal erreichbaren Erwartungswert des Steueraufkommens. Umgekehrt wird ein risikofreudiger Leviathan höhere Steuersätze erheben, solange er vermutet, daß er bei Erhöhung des Gesamtrisikos sein Steueraufkommen erhöhen kann.

1) Bitz (1981), S. 113

2) Dazu gehören z.B. Gleichverteilungen und Normalverteilungen. Vgl. Pawlowski $(1971)$, S. $153 \mathrm{ff}$.

3) Vgl. zu dieser Definition Pawlowski (1971), S. 50 oder Bamberg/Baur (1980), S. 126

4) In ökonometrischen Schätzungen wird die Normalverteilung von Zufallsvariablen sehr häufig unterstellt. Vgl. Schneeweiß (1978), S. $37 \mathrm{ff}$. 
Wie das Leviathan-Modell bei Sicherheit, liefert auch dieses Modell keinen umfassenden Ansatz für die Optimierung von Steuersätzen. Seine Schwächen liegen vor allem in der Vernächlässigung von Kreuzpreiseffekten und vielfältigen institutionellen Restriktionen, die für steuerpolitische Entscheidungen von größter Bedeutung sind. Deshalb steht nicht die Ableitung optimaler Leviathanregeln bei Unsicherheit im Vordergrund, sondern es geht vor allem um die Implikationen mangelnder Informationen für statiche Steuerentscheidungen, die im folgenden näher interpretiert werden.

\section{VI.3. Interpretation und Konsequenzen des Leviathan-Modells}

Wenn der Leviahtan, wie jeder aktuelle finanzpolitische Entscheidungsträger, realistischerweise unter Informationsmängeln leidet, muB davon ausgegangen werden, daß die Entscheidungen über Höhe und Struktur der Monopol-Steuersätze von jenen abweichen, die im deterministischen Modell ein Steueraufkommensmaximum garantieren. Da man bei Politikern und Bürokraten i.d.R. Risikoscheu erwarten darf, spricht vieles dafür, daß die Konsumentenpreise bei Unsicherheit unter den Cournot-Preisen bei vollkommener Information liegen werden. Es ist daher zu vermuten, daB das steueraufkommen eines Leviathan, der keine vollkommenen Informationenbesitzt, zwischen dem maximal möglichen Steueraufkommen eines deterministi schen Modells und dem Steueraufkommen eines wohlwollenden Planers liegen wird. Die Gefahr der totalen Ausbeutung durch einen risikoscheuen und wenig informierten Leviathan dirfte somit geringer sein als sie im Leviathanmodell von Brennan/Buchanan eingeschätzt wird, auch wenn das Leviathan-Steueraufkommen normalerwe ise über das gesellschaftich optimale MaB hinausgehen wird.

Die vorliegenden Ergebnisse tragen zu einer positiven Steuertheorie insowe it be $i$, als sie verdeut lichen, daB klar strukturierte Steuerregeln selbst im Falle eines eindeutig zielorientierten Leviathan nicht zu erwarten sind. Variierende Rahmenbedingungen und Risikobereitschaft lassen in weiten Bereichen eher "zufällige" Steuerpolitik erwarten. Zufällig ist diese Politik allerdings nur im Ver- 
gleich zum deterministischen Modell. Bei Unsicherheit über das Nachfrageverhalten der Konsumenten kann sie dagegen rational sein. Ein risikoscheuer Leviathan wird auf Teile seines potentiell erzielbaren Steueraufkommens verzichten, wenn er durch ein risikoärmeres steuersystem sein Gesamtrisiko verringern kann ${ }^{1)}$. Dies resultiert aus der Tatsache, daß die unterschiedlichen Steuerbemessungsgrundlagen nicht nur durch den Erwartungswert des Steueraufkommens, sondern auch unter Berücksichtiqung des damit verbundenen Risikos bewertet werden.

Bedenkt man ferner, daß die Höhe und Struktur der Steuersätze in diesem Modell c.p. von der Risikoeinstellung des Entscheidungsträgers abhängen, wird auch erklärlich, warum unterschiedliche Steuersätze auch bei gleicher erwarteter Steuerpreiselastizität der Nachfrage aus einem rationalen Leviathanverhalten resultieren können. Gemäß (VI.20) ist dafür die Wirkung der Steuerbetragsvariation auf die Standardabweichung bzw. auf das RisikomaB R verantwortlich. Berücksichtigt man in diesem Zusammenhang, daß im steuerpolitischen Willensbildungsprozeß sehr viele Personen mit divergierender Risikoneigungmitwirken und die Mitwirkenden im Laufe des Verfahrens häufiger wechseln, sei es, weil sie abgewählt werden oder aber aufgrund der Fluktuation in den Mitarbeiterstäben ${ }^{2)}$, so sind Entscheidungen unter Unsicherheit selbst bei gleicher Information eine Quelle unsystematischer, subjektiver Entscheidungen. Diese "Zufälligkeiten" des steuerpolitischen Entscheidungsprozesses lassen kaum noch eine Prüfung der Frage zu, ab aktuelle Steuerreformen tatsächlich normbezogen sind. Dies um so mehr, wenn man bedenkt, daß die Korrelationen zwischen Märkten nur sehr unvollkommen einzuschätzen sind - und das selbst dann, wenn man, wie im vorliegenden Mode11, wesentliche Komplementaritäts-und Substitutionsbeziehungen zwischen den Märkten vernachlässigt und unterstellt, daß die nachgefragten Mengen der einzelnen Güter nur vom

1) Vgl. zur Interpretation des Risikomaßes R, das die Zunahme des Erwartungswertes mißt, die man mit einer gleichmäßigen Ausdehnung des gesamten optimal strukturierten Risikoportefeuilles erreichen kann, Sinn (1980), S. 275

2) So wechselte der Vorsitz im Assistant Secretary for Tax Policy in den USA zwischen 1968 und 1977 sechsmal. Vgl. Reese (1980), S. 14 
eigenen Preis abhängen. Die Prognostizierbarkeit staatlichen Handelns wird somit stark eingeschränkt.

\section{VI.4. Neue Regeln für den Leviathan?}

Aus diesen Implikationen ergeben sich einige wichtige finanzpolitische Konsequenzen: Je besser die Information der staatlichen Planer ist, um so kritischer hängt das Wohl und Wehe der Bürger von der wahren Zielsetzung der staatlichen Organe ab. Wohlwollende staatliche Planer werden die aggregierten Wohlfahrtsverluste minimieren, böswillige Diktatoren werden die Ausbeutung des Volkes perfektionieren können. Da es jedoch keine Möglichkeit zu geben scheint, eine Gesellschaft ein für allemal vor despotischen Regierungen zu bewahren, können Informationsdefizite staatlicher Organe grundsätzlich als Schutz vor staatlicher Ausbeutung interpretiert werden. Gerade optimale Kopfsteuern haben diesen JanusKopf. Sie erfordern ein Maximum an Informationen, da zu ihrer Optimierung nicht nur diverse Einkommensbestandteile, sondern auch individuelle Neigungen und Fähigkeiten der Zensiten durch die Finanzbehörde ermittelt werden müssen. Kopfsteuern ermöglichen somit einen unabwendbaren statlichen Zugriff. Im Falle wohlwollender Planer führt dies zu einem gesellschaftlichen Optimum. Im Falle eines Leviathan führt dies zur perfekten Ausbeutung. Aus diesem Grund erscheinen individuelle Kopfsteuern und Steuerreformvorschläge, die einen zu hohen Informationsbedarf haben, als ungeeignet. Das impliziert insbesondere das Verbot der Einzelerfassung individueller Fähigkeiten und Merkmale, an welche die maximalen Kopfsteuern geknüpft werden könnten. Damit ist auch die Notwendigkeit des Datenschutzes gegenüber dem Fiskus begründet.

In diesem Zusammenhang kann auch künftig die traditionelle Vorgehensweise verteidigt werden, nach der nicht die individuellen Nutzenniveaus selbst gemessen werden, sondernderen Indikatoren, die lediglich eine begrenzte Zahl persönlicher Daten erfordern; diese Indikatoren, wie z.B. Einkommen und Konsum sollten so gewählt werden, daB sie eine möglichst eindeutige Beziehung zuderwirtschaft- 
1 ichen Situation bzw. Nutzenposition des einzelnen Zensiten erwarten lassen. Das bedeutet jedochnicht, daß sie nach individuellen Präferenzen modifiziertwerden sollten, d.h. es sollte bei der Wahl $z$. B. des Einkommens als Steuerbemessungsgrundlage keine Rolle spielen, mit welcher Arbeitsfreude oder welchem Arbeitsleid es erwirtschaftet wurde, da zur Ermittlung dieser psychologischen Faktoren die gesamte Persönlichkeit des Zensiten durchleuchtet werden müBte. Ebenso sollte die Konsumtion gleicher Gütermengen durch verschiedene Individuen nicht unterschiedlich gewichtet werden, denn die hierfür notwendigen persönlichen Daten der Steuerzahler könnten von einem Monopolstaat zu weiterer Ausbeutung und Kontrolle mißbraucht werden. Die Begrenzung der Steuerbemessungsgrundlage auf marktmäßig bewertbare Größen, wie z.B. Einkommen oder Guiter, erfährt somit eine weitere Begründung.

Die vorangegangenen Argumente lassen Steuerreformregel 5 auch unter der realistischen Annahme unvollkommener Information uneingeschränkt bestehen. Verfassungsregeln sind eine notwendige Voraussetzung zur Einschränkung fiskalischer Willkuir und Unberechenbarkeit, die im Falle von Informationsdefiziten schon aus wechselnder Risikoeinstellung der Entscheidungsträger resultieren können.

Trotzdem lassen sich mit diesem Modelleine Vielzahl von systematischen Unzulänglichkeiten des Steuersystems nichterklären. Insbesondere ist es nicht möglich, die systemimmanenten Mängel des demokratischen Reformprozesses herauszufiltern. Einem umfassenden Erklärungsanspruch würde das Modell auch deshalb nicht gerecht, weil hier lediglich die Unsicherheitsaspekte ökonomischer Art untersucht werden, die ihrerseits nur einen Teil der komplexen Steuerreformabläufe darstellen. Aus denselben Gründen kann das Modell auch nicht als umfassender Optimierungsansatz gewertet werden. statt dessen wird der Versuch unternommen, im Sinne eines positiven Modellansatzes einige jener Komponenten herauszufiltern, die fürdie steuerpolitischen Entscheidungen bei Unsicherheit von kritischer Bedeutung für den Fiskus sein können und daher auch immer wieder in Steuerreformprozesse eingeflossen sind. Daneben könnten die Ergebnisse auch als Entscheidungshilfen für einen un- 
vollkommen informierten Leviathan interpretiert werden. Die vielfältigen Steuervergünstigungen, Steuerbefreiungen, Subventionen und niedrigen Steuersätze bei Grundnahrungsmitteln sind jedoch eindeutige Belege dafür, daß die Politiker in der Realität keine Leviathanstrategie verfolgen (können). Der Hinweis auf Informationsmängel würde hier keine Erklärung liefern, da jede Einengung der Steuerbemessungsgrundlage, auch bei unvollkommener Information, das Ausbeutungspotential des Leviathan vermindert. Die Begrenzung der Steuersätze auf z.B. Grundnahrungsmittel scheint ebenfalls mit Leviathaninteressen kaum vereinbar, da es seit langem zum ökonomischen Allgemeinwissen gehört, daß diese Güter eine sehr unelastische Preiselastizität der Nachfrage besitzen.

Offensichtlich ergeben sich für den Fiskus neben den Informati-. onsdefiziten weitere finanzpolitische Restriktionen, wie z.B. wahltaktische oder verteilungspolitische Zwänge, die er im laufenden demokratischen Willensbildungsprozeß berücksichtigen muß. Zur Erkiärung aktueller Steuerpolitik erscheint es somit notwendig, zusätzlich jene Einflüsse seitens konkurrierender Parteien, der Steuerzahler und anderer Gruppen zu analysieren, welche den steuerpolitischen Entscheidungsprozeß mitprägen. 
VII. ZUR POSITIVEN FUNDIERUNG STAATLICHEN VERHALTENS IM POLITISCH-OKONOMISCHEN STEUERREFORMPROZESS

Im vorangegangenen Kapitel konnte demonstriert werden, da $\beta$ die Ableitung und Beurteilung steuerpolitischer Empfehlungen nicht nur von der Steuerbemessungsgrundlage, dem Steuerinstrumentarium und den Präferenzen der Zensiten abhängen, sondern auch von dem Verhalten bzw. von der Zielfunktion des Fiskus. Vor der Ableitung von Optimalregeln sollte daher ein realistisches Bild der politischen Entscheidungsträger sowie der Entscheidungsmechanismen gezeichnet werden ${ }^{1)}$. Die Vorstellungen von wohlwollenden oder böswilligen Diktatoren erfüllen diese Voraussetzungen nicht. Obwohl die Modelle aus theoretischer Sicht sehr aufschluBreich sein mögen, sind sie kein adäquater Spiegel der Realität. In der vorliegenden Arbeit werden sie daher auch als ökonomische Extrempositionen ohne politischen Inhalt interpretiert, deren Ergebnisse allein auf ökonomischen Gegebenheiten basieren. Tatsächlich aber werden steuerpolitische Entscheidungen nicht nur von ökonomischen, sondern auch von politischen $Z$ wängen beeinfluBt ${ }^{2}$ ). Im folgenden soll daher die Frage beantwortet werden, welche Faktoren dem politischökonomischen Steuerreformprozeß die wesentlichen Impulse

1) Diese Forderung hat in einem allgemeineren, philosophischen aber auch wirtschaftswissenschaftlichen Rahmen eine lange Tradition. Schon Machiavelli traf "die grundlegende und berühmte Unterscheidung zwischen dem 'wirklichen Wesen der Dinge' und den bloß 'vorgestellten Freistaaten und Alleinherrschaften, von denen man in Wirklichkeit weder etwas gesehen noch gehört hat'. Damit ist gesagt, daß Moral und politische Philosophen bis dahin ausschließlich über letztere geredet und dabei versäumt hatten, Richtlinien für die reale Welt zu liefern, in welcher der Fürst handeln mußte". Hirschmann (1977), S.21

2) Dies bedeutet, daß keine optimale Reformstrategie sondern nur Reformtaktiken entwickelt werden. Vgl. Z.B. Shoup (1970), S. $345 \mathrm{ff}$., Neumark (1971), S. $152 \mathrm{ff}$. Ein typisches und in jüngster Zeit viel diskutiertes Beispiel für solche Zwänge sind die "tax revolts" in den Vereinigten Staaten. Vgl. Folkers (1983a) und die dort angegebene Literatur. Von gröBter Bedeutung ist aber auch der heimliche, illegale Steuerprotest, nämlich das Ausweichen der Steuerbürger in die Schattenwirtschaft. Vgl. Z.B. Neumark (1981), S. 385 
verleihen ${ }^{1)}$. Erst in diesem Zusammenhang kann geklärt werden, welche der konstitutionellen Steuerregeln für den Leviathan und gleichermaßen für den demokratischen Steuerstaat Guiltigkeit besitzen.

Die positive Analyse von Steuerreformen erfolgt daher im politisch-ökonomischen Rahmen. In Abb. 3 sind alle an der Steuerreform beteiligten Aktoren aufgeführt. Die Beziehung zwischen den einzelnen Gruppen wird mit Pfeilen dargestellt und weist eine wesentlich komplexere Struktur auf als jene im reinen OT-Modell (vgl. Abb. 1). Um den Rahmen dieser Arbeit nicht zu sprengen, werden im folgenden zwei Untersuchungsschwerpunkte gebildet. Im Mittelpunkt des Interesses stehen alternative Hypothesen über Verhaltensweisen der Regierung und der Zensiten, die sukzessive in den folgenden Abschnitten analysiert werden. Dagegen werden die übrigen in Abb. 3 aufgezeigten Einflusse nur insoweit dargestellt, als sie fur die Untersuchung von Bedeutung sind.

Der politisch-ökonomische Ansatz (vgl. Abb.3) dient dazu, prinzipielle Mängel und Zusammenhänge im politisch-ökonomischen Verlauf von Steuerreformprozessen aufzudecken. Die Erklärung oder Prognose bestimmter Steuergesetze sind, wenn überhaupt, nur im konkreten Einzelfall möglich und somit nicht Gegenstand dieser Untersuchung. Nach der Diagnose der politischen Institutionen kann dann eine Therapie vorgeschlagen werden, die nicht nur die Symptome sondern die Ursachen der Fehlentwicklung im steuerpolitischen Bereich bekämpft. ${ }^{2}$ )

Im folgenden soll zunächst die Bedeutung der finanzpolitischen Entscheidungsträger und der Parteienkonkurrenz im steuerpoli-

1) $\mathrm{Vgl.Z}$.B. zu einer ersten Diagnose der Reform zum Einkommensteuertarif für 1979 Folkers (1978), insbesondere S. $343 \mathrm{ff}$. und Folkers (1983b), S. $189 \mathrm{ff}$.

2) Vgl. zu einer ähnlichen Interpretation Folkers (1983c), S. 168 


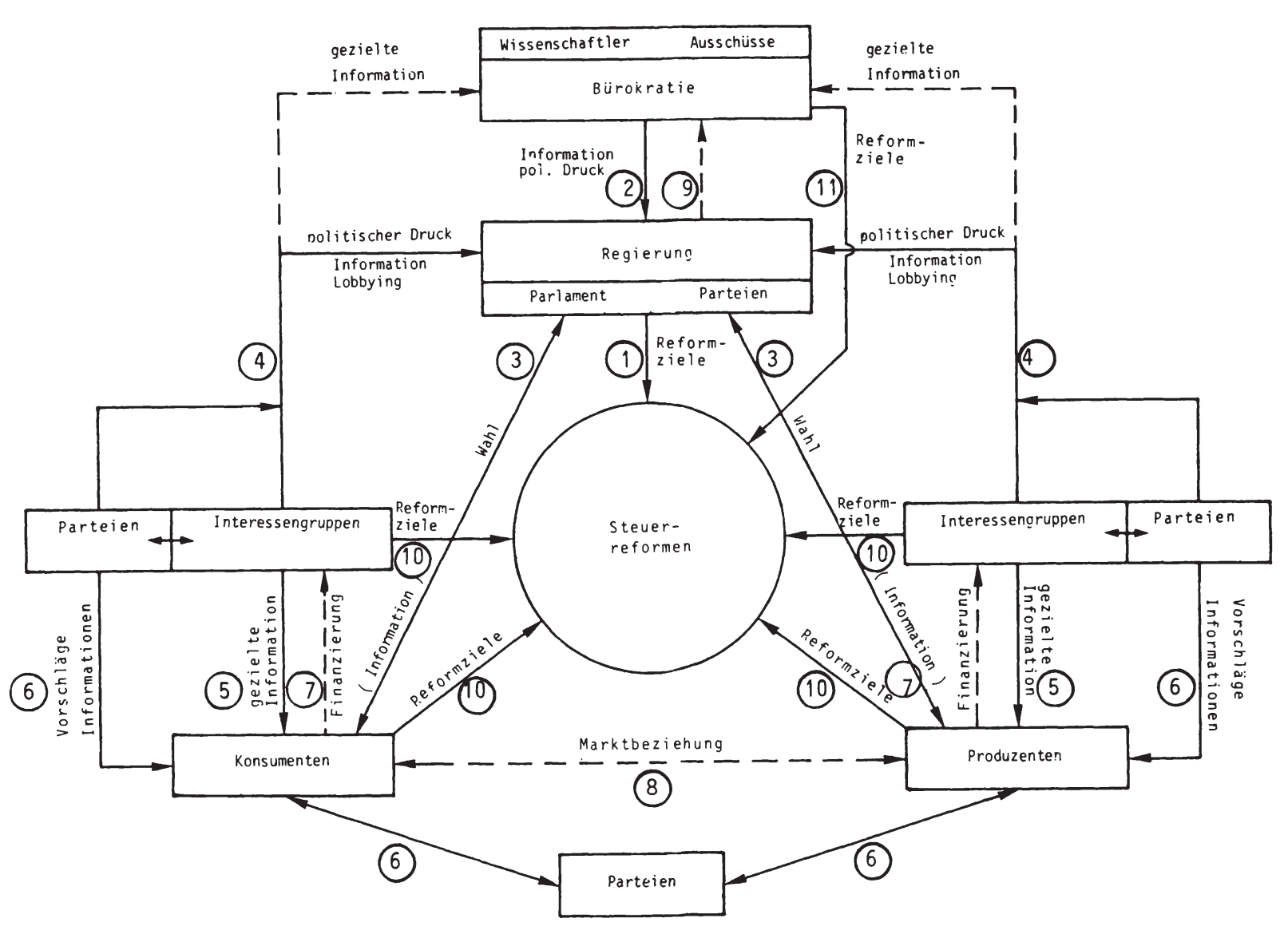


tischen willensbildungsprozeB analysiert werden. Im AnschluB daran rückt die aktive Rolle der Steuerzahler bei der Gestaltung der Steuergesetzgebung in den Mittelpunkt der Untersuchung.

VII.1. Die Bedeutung fiskalischer Restriktionen für den finanzpolitischen EntscheidungsprozeB

Nachdem im vorangegangenen Kapitel nachgewiesen wurde, daB die perfekte Ausbeutung der Zensiten schon aus informationstechnischen Gründen recht unwahrscheinlich anmutet, und auch ein empirischer Beleg für die These nicht erbracht werden konnte ${ }^{1}$, stellt sich die Frage, inwieweit die simplen Verhaltenshypothesen der Leviathan-bzw. OT-Modelle aus politisch-ökonomischer Sicht modifiziert werden müssen, um die Ergebnisse des aktuellen steuerpolitischen Willensbildungsprozesses besser erklären zu können. Zu diesem Zweck wird zunächst untersucht, durch welche Restriktionen die Entscheidung der Politiker eingeengt wird (vgl. Abb. 3, Pfeil 2,3 sowie 4) und welche Möglichkeiten für sie bestehen, dennoch finanzielle Handlungsspielräume ${ }^{2)}$ für ihre Eigeninteressen zu schaffen. In diesem Zusammenhang läßt sich zeigen, daß der demokratische Willensbildungsprozeß weder die Entstehung begrenzter finanzieller Handlungs spielräume für Politiker und Bürokraten noch unsystematische steuerstrukturen verhindern kann.

1) Vgl. Z.B. die Ausführungen in Abschnitt V.6

2) Der Begriff "finanzieller Handlungsspielraum" wird hier und im folgenden in Anlehnung an Folkers (1983b), S. 202 definiert, d.h. "Das unmittelbare instrumentale Ziel der Politiker im Steuerreformprozeß ist die Sicherung einer satisfizierenden Finanzmasse zur Realisierung der unterschiedlichen, ihren Eigeninteressen förderlich erscheinenden Gruppenvorteile im Steuer- und Ausgabenbereich (...). Der politische Sektor ist im Reformprozeß an einem ergiebigen Steuersystem interessiert, das den notwendigen finanziellen Handlungsspielraum gewährt". 
VII.1.1. Fiskalische Restriktionen in der Demokratie

Die Idee einer Finanzverfassung ist nicht neu. Restriktionen der Besteuerung sind in beinahe allen Verfassungen verankert ${ }^{1}$. Sie wurden einaeführt, weil bereits die Verfassungsväter moderner Demokratien die Gefahr eines unkontrollierten Steuerstaates erkannt hatten. Insofern kann die konstitutionelle Theorie der Besteuerung auch als positive Steuertheorie verstanden werden. Beispielsweise enthält die deutsche Finanzverfassung explizit das Gebot der horizontalen Gerechtigkeit ${ }^{2}$, eine Regel, die Brennan/Buchanan auch aus normativer Sicht fordern würden ${ }^{3)}$.

Neben dem geschriebenen Verfassungsrecht gibt es auch einen ungeschriebenen Konsens über die vor allem im fiskalischen Sinne wichtigen Grobstrukturen eines Steuersystems. Beispiele dafür sind progressive Einkommensteuern und proportionale Verbrauchsteuern, die nach Brennan/Buchanan ebenso als Steueraufkommensrestriktionen verstanden werden können ${ }^{4}$ ), ohne daß diese Steuern verfassungsrechtlich verankert wären ${ }^{5}$ ). Für die Beständigkeit verteilungspolitisch und fiskalisch bedeutsamer Steuerstrukturen mögen einerseits verwaltungstechnische Gründe von großer Bedeutung sein, andererseits aber sind mit solchen großen Steuerreformen zwangsläufig erhebliche Anderungen der volkswirtschaftichen Rahmenbedingungen verbunden, welche

1) Vgl. Buchanan (1979), S. 350 f. oder für spezifische Ausführungen zur Bundesrepublik Deutschland Benda/Kreuzer (1973), S. 50, Stern (1980), S. $1105 \mathrm{ff}$.

2) Vgl. Stern (1980), S. 1109

3) Vgl. Brennan/Buchanan (1980), S. 36

4) Vgl. Brennan/Buchanan (1980), S. 47

5) Diese Feststellung gilt natürlich auch für andere wichtige Steuern, wie z.B. Körperschaftsteuer, Vermögensteuer u.a., die hier nicht explizit erwähnt werden. 
$\mathrm{zu}$ wachsenden Risikokosten ${ }^{1)}$ und zur Opposition der Steuerzahler führen können ${ }^{2)}$. Aufgrund der ungewissen Reaktionen der Steuerzahler nach einer großen Reform, kann es sowohl zu unerwünschten Schwankungen des Steueraufkommens als auch zu merklichen, von der Regierung jedoch nicht kalkulierbaren Verschiebungen der Steuerlastverteilung kommen.

Daruber hinaus erschwert die hohe Publizität großer Reformen eine willkürliche Umverteilung von individuellen Steuerlasten zugunsten des Fiskus oder einzelner Bevölkerungsgruppen ${ }^{3}$. Die politische Konkurrenz kann unerwünschte Folgewirkungen der Reform wählerwirksam vermarkten und die Wiederwahlchancen der Regierung schmälern. Infolge der UngewiBheit über die ökonomischen Konsequenzen großer Reformen wird die Regierung das Risiko des Mißerfolges auf möglichst viele Schultern verteilen und deshalb auch die Zustimmung der Opposition einholen wol1 le ${ }^{4)}$. Die Beständigkeit nominaler Grobstrukturen des Steuersystems beruht somit nicht auf ethischen Forderungen, sondern in erster Linie auf einem politischen Rationalkalkiil. Die Unsicherheit über die politischen und ökonomischen Reaktionen der Steuerzahler ist auch hier, wie bereits im letzten Kapitel deutlich wurde, eine der bedeutendsten Restriktionen. Die skizzierten Zusammenhänge belegen ein Konsenspotential in der Steuerpolitik und einen Anwendungsbereich der konstitutionellen Theorie der Besteuerung, der vor allem in den existierenden Finanzverfassungen manifestiert ist.
1) Vgl. Alt (1983), S. 187
2) Vgl. dazu Miner/Chalice (1978), S. 61
3) $\mathrm{Vgl}$. Ben-Porath/Bruno 1977
4) Vgl. Alt (1983), S. 184 


\section{VII.1.2. Geltende Finanzverfassungen}

Konstitutionellen Finanzgesetzen als Barrieren gegen statiche Willkür wurden in einzelnen Ländern unterschiedliches Gewicht be igemessen.

In der Bundesrepublik Deutschland "haben allgemeine Verfassungsnormen, insbesondere Grundrechte und rechtsstatliche Prinzipien für die Beziehungen des steuerzahlenden Bürgers zum Gemeinwesen maßgebliche Bedeutung erlangt" 1 ). Als verfassungsrechtiche Grenzen der Besteuerung sind in der Bundesrepublik Deutschland folgende Grundrechte wichtig:

das Recht auf freie Entfaltung der Persönlichkeit (Art.2,Abs.1). der Gleichheitsgrundsatz (Art.3),

die Glaubens- und Bekenntnisfreiheit (Art.4), der Schutz von Ehe und Familie (Art.6, Abs.1). die Berufsfreiheit (Art.12,Abs.1) und die Eigentumsgarantie $\left.(\operatorname{Art.14})^{2}\right)$.

Die am häufigsten angeführte konstitutionelle Steuerregel ist das Prinzip der Gleichmäßigkeit der Besteuerung auf der Grundlage der individuellen Leistungsfähigkeit, das auf dem Gleichheitssatz des Art.3, Abs. 1 GG aufbaut ${ }^{3)}$. Eine ganz ähnliche fiskalische Begrenzungsregel ist die uniformity rule, die in Article 1, Section 8 der Verfassung der USA verankert ist. Diese Regel fordert: "all Duties. Imposts and Excises shall be uniform throughout the United States"4). Die allgemeine Rechtsnorm "Gleichheit vor dem Gesetz", wird in dieser Hinsicht auch auf die Besteuerung ausgedehnt ${ }^{5}$ ).

1) Benda/Kreuzer (1973), S. 51. Vgl. auch Kirchhof (1973), S. $10 \mathrm{ff}$.

2) Benda/Kreuzer (1973), S. 52. Die Bedeutung der einzelnen Grundrechte für die Begrenzung der Besteuerung werden dort ausführlich dargestellt. $\mathrm{Vgl}$. auch Kirchhof (1973) und Piduch (1964).

3) Vgl. Stern (1980), S. 110 oder Folkers (1984), S. 500 f.

4) Zitiert nach Musgrave/Musarave (1984), S. 33

5) Buchanan (1979), S. 351 
Mit dieser Begrenzung zulässiger Steuerstrukturen ist gleichzeitig eine Begrenzung des Steueraufkommens verknüpft, weil Mehrheiten und Minderheiten in der Bevölkerung stets in gleicher Weise zur Finanzierung öffentlicher Güter beitragen ${ }^{1)}$. Die Ausbeutung von Minderheiten durch Mehrheiten kann damit zumindest eingeschränkt werden. Allerdings erscheint die gegenwärtigguil tige Interpretation des Gleichheitsgrundsatzes nach dem Prinzip der wirtschaftlichen Leistungsfähigkeit recht problematisch, weil das Leistungsfähigkeitsprinzip selbst keine materiellen Gestaltungsvorschriften beinhaltet. Dadurch wird der Gleichheitsgrundsatz unbestimmt und verliert weitgehend seine Funktion als Begrenzungsrege ${ }^{2}$ ).

Neben dem Prinzip der Gleichmäßigkeit der Besteuerung sind weitere fiskalische Regeln "z.T. in der Finanzverfassuna, zum anderen Teil in zahlreichen Einzelgesetzen niedergelegt, die jedoch selten als Ganzes kodifiziert werden" ${ }^{3)}$. In der Finanzverfassung ${ }^{4}$ werden unter anderem auch die Grundlagen der Besteuerung und des Eigentumsrechts teils auf Verfassungsebene, teils in Einzelgesetzen geregelt. In diesem Zusammenhang ist die Abgabenordnung ( $A O$ ) vom 16.3. 1976 von großer Bedeutung, weil sie eine Vielfalt grundsätzlicher Steuerrichtlinien enthält ${ }^{5}$. $0 b-$ wohl die $A O$ als Mantelgesetzkonzipiertwurde und somit als zusätzlicher Schutz gegen staatliche Willkür interpretiert werden kann, fehlt ihr doch der konstitutionelle Charakter. Entfällt aber die Verabschiedung von Steuerregeln mit "relativer Einstim-

1) Brennan/Buchanan (1980), Kap. 3 und 4

2) Folkers (1984), S. 501

3) Hedtkamp (1977), S. 40

4) Zur Finanzverfassung und Gesetzgebung gehören das Haushaltsrecht, im allgemeinen das Schulden- und Vermögensrecht, Grundlagen der Besteuerung, die Eingriffe in den Besitzstand der Staatsbürger (vor allem Enteignung) und die Regelung der rechtlichen, vor allem finanzwissenschaftlichen Beziehungen zwischen den Gebietskörperschaften (Finanzausgleich). Vgl. Hedtkamp (1977), S. 40 und Abgabenordnung (1977).

5) Vgl. dazu Mittelsteiner/Schaumburg (1977) 
migkeit", d.h. einem erhöhten Quorum, so ist nicht mehr gewährleistet, daß mit Anderungen der politischen Machtverhältnisse die Schutzfunktion der fiskalischen Regeln erhalten bleibt. Bei würdigung der bestehenden Vorschriften bedarf es noch gröBerer Anstrengungen, $b$ is in allen Bereichen der Besteuerung tatsächlich optimale konstitutionelle Steuerregeln verfügbar sind. Das existierende Verfassungsrecht erfüllt diese Anforderungen nur unvollkommen. Die angeführten Beispiele demonstrieren, daB die bloBe Existenz einiger Verfassungsregeln noch nicht ausreicht, um den Staat effektiv zu beschränken; hierfür sind sehr viel präzisere Regelungen notwendig, die den Interpretationsspielraum des Fiskus minimieren.

VII.1.3. Weitere Restriktionen staatlicher Steuerreformentscheidungen

Neben den ökonomischen und steuerrechtlichen Restriktionen existieren weitere Hemmnisse und Nebenbedingungen im politisch-ökonomischen Steuerreformproze $B$, die den Handlungsspielraum der Politiker gravierend beschneiden.

Eine wesentliche Beschränkung erfahrendie politischen Akteure durch periodische Wahlen, die eine Minimalkonkurrenz auf der politischen Ebene implizieren und dem Steuerzahler eine Mindestkontrolle garantieren ${ }^{1)}$. Zweitens werden die Entscheidungen der Legislative durch verschiedene Grundrechte und Verfassungsregeln, wie z.B. das Recht auf freie Meinungsäußerung oder Auswanderung, restringiert ${ }^{2)}$. Eine dritte Beschränkung erfährt das staatliche Handein durch den dezentralen Aufbau des Staatsapparates. Die Dezentralisierung erzwingt steuerpolitische Entscheidungen auf konkurrierenden staatlichen Ebenen. Je näher die Entscheidungseinhei-

1) In föderativen Demokratien wird die Kontrollfunktion durch permanente Wahlen auf Bundes-, Länder- und Gemeindeebene noch wesentlich verstärkt.

2) Vgl. hierzu z.B. Bernholz (1972), S. $211 \mathrm{ff}$. 
ten am Bürger sind, um so geringer wird die Gefahr, daß ihm überhöhte Steuerzahiungen für unbekannte Zwecke aufgebürdet werden ${ }^{1}$ ). Viertens verhindert neben einem föderativen Staatsaufbau auch der heterogene Machtapparat das uneingeschränkte Walten der Regierung ${ }^{2}$. Die verantwortlichen Regierungsmitglieder müssen nämlich nicht nur Rücksicht auf die verschiedenen Flügel ihrer eigenen Partei nehmen ${ }^{3)}$, sondern auch auf die Wünsche und Vorarbeiten der sie unterstützenden Bürokratie (vgl. Abb. 3. Pfeile 3 und 4). Darüber hinaus müssen viele Reformgesetze von Bundes- und Länderparlamenten verabschiedet werden. Wenn in diesen Parlamenten verschiedene Parteien die Mehrheit haben, sind nur konsensfähige Reformen möglich. Von größter Bedeutung sind schließlich fünftens die Aktivitäten von organisierten Interessengruppen ${ }^{4}$, die die Entscheidungen der Regierung durch gezielte Informationen und adäquate Strategien zu beeinflussen suchen ${ }^{5)}$. Darüber hinaus sichern sich viele Verbände durch Spenden an Parteien und die Abordnung eigener Interessenvertreter in die Parlamente ein Einflußpotential, das sie vor staticher Steuerausbeutung schuitzt.

\section{VII.1.4. Die staatliche Zielfunktion}

Wenn sich weder Leviathan- noch OT-Regeln, im folgenden auch Optimierungsregeln genannt, mit aktuellen Steuerregeln decken, sind dafür in erster Linie die eben erläuterten Restriktionen verantwortlich. Gleichzeitig mag aber auch die staatliche Zielfunktion nicht korrekt spezifiziert sein, d.h. die Vorstellung

1) Vgl. Bernholz (1972), S. 214 und $226 \mathrm{f}$.

2) Vgl. Z.B. Good (1980), Kap. 3 und 4

3) Die Auswirkungen von Restriktionen der Parteimitglieder und Geldgeber auf die Parteien, die um Regierungsämter konkurrieren, werden bei Bernholz (1975), S. $32 \mathrm{ff}$. untersucht. In diesem Modell wird u.a. auch erklärt, warum die Wahl einer Partei möglich ist, obwohl sie einen Teil der Steuergelder für eigensüchtige Zwecke der Parteimitglieder verausgabt.

4) Zur Entstehung von Verbänden aufgrund politischer Entscheidungen und zur Erlangung von Vorteilen für die Verbandsmitglieder siehe 01 son (1968).

5) Zum Einfluß von Verbänden in einer Demokratie siehe beispielsweise Bernholz (1975), S. $96 \mathrm{ff}$. und insbes. Kap. VIII dieser Arbeit. "A tax law is always a compromise among the views of powerful individuals and groups". Pechman (1977), S. 32 
von ausbeutenden (oder wohlwollenden) Steuerstaten spiegelt die Motivationsstruktur aktueller Staatswesen nur unvollkommen wider. So muB im Gegensatz zum Konzept der OT nicht nur den Konsumenten und Produzenten, sondern konsequenterweise auch den Berufspolitikern und Regierungsmitgliedern, Nutzenmaximierung unterstellt werden ${ }^{1)}$. Das Nutzenniveau selbst kann von den verschiedensten Variablen wie z.B. Einkommen, Macht, Amtsprestige oder ideologischem Sendungshewußtsein abhängen. Diese Argumente der Nutzenfunktion müssen aber nicht prinzipiell positiv mit einem steigenden Steueraufkommen korreliert sein ${ }^{2}$, wie es im Leviathan-Modell implizit unterstellt wird. Auf po1 itischer Ebene sind sie dagegen beinahe ohne Ausnahme mit der Verleihung politischer Amter verbunden, welche durch Wahlen erobert werden müssen ${ }^{3)}$. Das Handeln der Regierung wird deshalb nicht einseitig auf Steueraufkommensmaximierung ${ }^{4}$, soridern vorrangig auf stimmengewinne ausgerichtet sein ${ }^{5) 6}$ ).

1) Vgl. Z.B. Buchanan (1967), S. 12, McKean (1964), oder Bartlett (1973)

2) Vgl. zu weiteren Argumenten Musgrave (1981a), S. 92

3) Vg1. Z.B. Downs (1957), S. 28, Breton (1978), S. 54 ff.

4) Brennan/Buchanan (1977), S. 256

5) Vgl. Downs (1957), S. 31. Für Downs ist Stimmenmaximierung das erste Ziel der Regierung. Bartlett nennt drei Gründe für die Stimmenmaximierungshypothese. Erstens die Unsicherheit der Regierung über die Wählerreaktionen und den Wunsch der Regierung nach Wiederwahl, zweitens wächst mit der Stimmenzahl das Machtpotential der Regierung und drittens steigen die Wiederwahlchancen der Partei, mit der sich viele Wähler bereits in der vergangenen Wahl einmal identifiziert haben. Vgl. auch Herder-Dorneich (1959), S. 52 ff., Frey (1981), S. 136, Bartlett (1973), S. 19. Bartlett geht daher bei seiner Analyse von der Stimmenmaximierungshypothese aus. Bartlett (1973), S. 26

6) Im Zusammenhang mit der zunehmenden Kreditfinanzierung öffentlicher Ausgabenprogramme diskutiert Baum (1982), S. $18 \mathrm{ff}$. und $129 \mathrm{ff}$. die Frage, inwieweit die finanzpolitischen Entscheidungsträger durch die Präferenzen der Wähler in ihrem Bemuihen um höhere Budgetdefizite restringiert werden. Auch Baum kommt zum Schluß, daß vor allem beim Herannahen des Wahltermins die Wählerwünsche in gewissem Umfang berücksichtigt werden müssen. Baum (1982), S. 20 
(Vgl. Abb. 3, Pfeil 3). Stimmenmaximierung wird somit ein eigenständiges Ziel der Regierung darstellen, wenn sie vorrangig ihre politische Macht ${ }^{1}$ ) erhalten will, durch die sie letztlich in die Lage versetzt wird, höhere Steuereinnahmen zu er$z$ wingen. Dieser These kommt vor allem Bedeutung $z u$, wenn anzunehmen ist, daß die Zahl der Stimmen eng mit der wirtschaft1 ichen Situation der wähler und diese wiederum mit den steuerpolitischen Entscheidungen der Regierung verknüpft ist. Die Politiker sind in diesem Falle bis zu einem gewissen Grade auch an die Präferenzen der Wähler gebunden ${ }^{2}$; das gilt selbst dann, wenn ein Trend zu höheren Steuereinnahmen beobachtbar ist ${ }^{3}$ ). (In Abb. 3 impliziert Pfeil 1 die Zielfunktionen bzw. die Wïsche und den Handlungsspielraum der Regierung.)

Das Ringen um einen Wahlsieg (und damit um ein gewisses Stimmenpotential) kann aber auch als Restriktion verstanden werden, die erfüllt sein muß, um in ein bestimmtes Amt zu gelangen. Beispielsweise könnte sich ein Politiker schon mit einer einfachen Mehrheit zufriedengeben, wenn damit das gewüschte Amt gesichert bleibt. Die Stimmenzahl, die ihm die einfache Mehrheit garantiert, ist dann die Nebenbedingung, unter der er seinen Nutzen maximiert.

Im folgenden soll daher überprüft werden, inwieweit das fiskalische Ziel der Regierung durch Wahlrestriktionen tangiert wird.

1) Politische Macht bzw. Handlungsfreiheit der Politiker drückt sich darin aus, einen möglichst großen Teil der eigenen Wünsche und politischen Vorstellungen in soziale Realität transformieren zu können.

2) Vgl. zu einer Analyse der Politiksteuerung durch Wahlen Preusker (1974). Nach seiner Auffassung wird gerade die Steuerpolitik durch Wahlen stark beeinfluBt. Preusker (1979), S. 121. Val. auch Steuerreformkommission (1971), S. 28. Ein Beispiel aus USA im Zusammenhang mit der Abschaffung von "farm-tax-1oopholes", "Truman came out and said: This is a hell of a good paragraph, I wish we could use it. But this is an election year. Te11 John (Snyder) we'11 use it next year". Reese (1980), S. 29

3) Diese Tendenz wird auch durch das immer wieder erklärte Interesse der Politiker am fiskalischen Ziel unterstrichen. Vgl. Z.B. Zimmermann (1977). Vgl. zur Diskussion staatlichen Verhaltens in bezug auf Budgetdefizite Baum (1982) 
VII.1.5. Parteienwettbewerb und wachsender politischer Handlungsspielraum

VII.1.5.1. Das relative Wachstum von Ausgaben und Einnahmen des Staatssektors aus empirischer sicht

Das relative Wachstum der Staatsausgaben und der Steueraufkommen in allen westlichen Demokratien läßt sich empirisch nachweisen. Für die vorliegende Untersuchung sind hauptsächlich die Entwicklungen der vom Staat insgesamt kontrollierten Ausgaben $\left(A_{S T}\right.$ ) und Steuereinnahmen $(T)$ von Bedeutung; sie spiegeln das Ausmaß staatlicher Aktivität und Steuermacht wider. Als MeBziffer für die staatliche Ausgabentätigkeit und Wirtschafts macht steht beispielhaft die Gesamtausgabenquote der Bundesrepublik Deutschland ${ }^{1)}$ in Tab. 1 zur Verfügung, wobei BSP das Bruttosozialprodukt darstellt. Tab. 1 gibt die steigende Tendenz dieses Strukturkoeffizienten wider ${ }^{2}$.

\begin{tabular}{|c|c|c|c|c|c|c|c|c|}
\hline Jah. ${ }^{31}$ \\
\hline A $_{\text {ST }}$ /BSP & 0,325 & 0,368 & 0,379 & 0,477 & 0,471 & 0,497 & 0,498 & 0,489 \\
\hline
\end{tabular}

Entwicklung der Statsausgabenquote in der Bundesrepublik Deutschland

Die Macht der Besteverung kommt durch die Steuerquote T/BSP zum Ausdruck ${ }^{4)}$. Dazubenötigt man ${ }^{5)}$ die steuerlichen Einnahmen imwei teren Sinne, die neben den Steuern im engeren Sinne Gebühren und

1) Vgl. zur Diskussion dieser unechten Quoten z.B. Littmann (1975), S. 66 $\mathrm{ff}$. oder Folkers (1979), S. $457 \mathrm{f}$.

2) Derselbe Trend läßt sich auch für andere Länder nachweisen. Vgl. Z.B. Musgrave/Musgrave (1984), S. 138

3) Vgl. Folkers (1979), S. 460

4) Vgl. Littmann (1975), S. 77 und Folkers (1979), S. 459

5) Zu einer anderen Interpretation siehe Dreißig (1984), S. 173 
Beiträge, einschließlich der Sozialversicherung, beinhalten. Sie entsprechen dem "Anteil des Sozialprodukts, der den Privaten nicht unmittelbar zur Verfügung steht, sondern zunächst durch Zwang vom Staat an sich gezogen wird"1).

Die Entwicklung der Steuerquote in der Bundesrepublik Deutschland, die aus Tab. 2 ersichtlich wird, weist einen steigenden Trend $a u f^{2)} 3$ ).

\begin{tabular}{|c|c|c|c|c|c|c|c|c|}
\hline Tahr & 1960 & 1965 & 1970 & 1975 & 1980 & 1981 & 1982 & 1983 \\
\hline T/BSP & 0,333 & 0,341 & 0,365 & 0,408 & 0,423 & 0,424 & 0,424 & 0,421 \\
\hline
\end{tabular}

Entwicklung der Gesamtsteuerquote in der Bundesrepublik Deutschland.

VII.1.5.2. Ein Leviathán-Reformmodell unter Berücksichtigung von Wählerinteressen

Im folgenden soll untersucht werden, welche Steuerstrukturen von Regierungen angestrebt werden, die das fiskalische Ziel verfolgen, aber gleichzeitig durch Wählerpräferenzen restringiert sind ${ }^{5)}$. Grundlage der folgenden Analyse ist wiederum das OT-Modell in Kap. II, in welchem die Regierung für die schrittweise Erhöhung der Wohlfahrt bei gleichem Steueraufkommen unter ausschließlich ökonomischen Nebenbedingungen zu sorgen hatte. Im Gegensatz zu den institutionellen Implikationen des OT-Modells wird jetzt aber unterstellt, daß der Fiskus vorrangig das fiskalische Ziel im Auge hat und durch die

1) Folkers (1979), S. 459

2) Die Daten in Tab. 2 entsprechen jenen aus Tab. 4 bei Folkers (1979), S. 460. Vgl. zur Gültigkeit dieser Aussage für die Länder der EG bis 1978 Caesar (1980), S. 11, Ubersicht 26

3) Dieser Trend würde bei einer eingeschränkten staatlichen Nettokreditaufnahme sicherlich erheblich verstärkt. Vgl. zur übersicht über die Schuldenentwicklung europäischer Länder die Finanzberichte der Bundesregierung.

4) Die Steuerquoten aus Tabelle 2 wurden gemäß den Daten aus Fachserie 18 Reihe 6 "Der Staat in den volkswirtschaftlichen Gesamtrechnungen", 1960 bis 1983, S. 40 berechnet.

5) Vgl. zu ähnlichen Oberlegungen Folkers (1983b), S. $203 \mathrm{ff}$. 
in den vorangegangenen Abschnitten diskutierten Faktoren des politischen Prozesses restringiert wird. Dabei wird unterstellt, da $\beta$ modellexogene Beschränkungen die im Parteienwettbewerb stehende Regierung zwingen, das Nutzenniveau eines repräsentativen Konsumenten keinesfalls sinken zu lassen, wenn sie ihre Wiederwahl nicht gefährden will. Andererseits ist sie aber bestrebt, ihren Handlungsspielraum durch stetige Steuereinnahmenerhöhungen auszubauen. Obwohl im Rahmen dieses Grundmodells keine weiteren politisch-ökonomischen Restriktionen endogenisiert werden können, wird durch den erläuterten Optimierungskalkül ein realistischeres Bild der politischen Akteure und Restriktionen gezeichnet als im OT-Reformmodell.

Unter den dargestellten Voraussetzungen kann der Optimierungsansatz für das Modell eines repräsentativen Steuerzahlers wie folgt beschrieben werden:

Erhöhe das Steueraufkommen

(VII.1) $d T>0$

unter der Nebenbedingung eines konstanten Nutzenniveaus

(VII.2) $d v=0$.

Das totale Differential von (VII.1) erhält man aus (V.7):

(VII.3) $d T=\sum_{i} \sum_{k} t_{i} \frac{\partial x_{i}}{\partial t_{k}} d t_{k}+\sum_{k} x_{k} d t_{k}+\sum_{i} t_{i} \frac{\partial x_{i}}{\partial m} d m-d m>0$.

Das totale Differential von (VII.2) stammt aus (II.4):

(VII.4) $d v=\sum_{i} \frac{\partial v}{\partial q_{i}} d q_{i}+\frac{\partial v}{\partial m} d m=0$.

Nach Einsetzen von (VII.4) in (VII.3) und einigen Umformungen folgt: ${ }^{1)}$

(VII.5) $d T=\sum_{i} \sum_{k} t_{i} s_{i k} d t_{k}>0$

und damit

1) Vgl. Anhang $E$ 
Leviathanregel $3:$

Gleichung (VII.5) ist identisch mit Gleichung (II.49) und besagt daher, daß zur schrittweisen Erhöhung des Steueraufkommens unter der Nebenbedingung konstanten Nutzens dieselben Steuerregeln wie im Falle einer OT-Steuerreform verfolgt werden sollten.

Das zunächst erstaunliche Ergebnis wird auch intuitiv verständlich, wenn man bedenkt, daß der Fiskus durch eine geschickte Besteuerung die Mehrbelastung der Steuerzahler senken kann und dafür maximal das monetäre Aquivalent des Nutzengewinns als zusätzliches Steueraufkommen abzieht. Voraussetzung dafür ist, daß der Leviathan derart an die Wählerinteressen gebunden ist, daß er keine (merkliche) Ausbeutung der Zensiten veranlassen kann. Ausgehend von einem unsystematisch gewachsenen Steuersystem ist es ihm lediglich möglich, eine Umstrukturierung der Steuerstruktur derart vorzunehmen, daß ein Steueraufkommenszuwachs realisiert wird, ohne die Wähler in ihrem Wohlbefinden zu beeinträchtigen. Betrachtet man den Leviathan als Teil der Gesellschaft, so impliziert seine fiskalische Zielsetzung gleichzeitig eine Wohlfahrtssteigerung nach dem Paretokriterium, weil sein Nutzen durch die zusätzlichen Steuern steigt, aber niemand sonst Nutzeneinbußen erleidet. Unterstellt man weiter, daß den Zensiten ein Teil des zusätzlichen Steueraufkommens aus irgendwelchen Gründen in Form von Gütern und Dienstleistungen wieder zugute kommt, so kann das Nutzenniveau aller Gesellschaftsmit-glieder steigen.

Fiskalisches Ziel und schrittweise Pareto-Verbesserungen müssen sich also nicht grundsätzlich widersorechen, auch wenn das Verteilungsergebnis, nämlich der Steuer-bzw. Nutzengewinn des Leviathan, aus der sicht des Steuerzahlers kaum wünschenswert ersche int ${ }^{1)}$.

1) Die Problematik besteht somit in einer als ungerecht betrachteten Verteilung, nicht in Allokationsverzerrungen. 
Die Modellergebnisse demonstrieren die Gefahr einer von den Wählern nicht kontrollierbaren und von der Ausgabenseite losgelösten Anwendung der OT-Regeln. Denn es bleibt zu fragen, ob man sicher sein kann, daß der Fiskus bei schrittweisen Steuerreformen unter Berücksichtigung der abgeleiteten Optimalregeln auch tatsächlich nur das Wohl des Steuerzahlers im Auge hat ${ }^{1)}$. Sofern das Steueraufkommen durch die Reform ansteigt, kann die Motivation des Fiskus nicht mehr eindeutig aus der optimierungsregel abgeleitet werden. Die Kontrolle $e^{2)}$ der politischen Entscheidungsträger wird dadurch erheblich erschwert. Nur aufkommensneutrale OP-Strukturänderungen deuten darauf hin, daß ausschließlich effizienzsteigernde Maßnahmen intendiert sind. Die neuerdings häufig geforderte konstitutionelle Steueraufkommensbegrenzung wird in diesem Licht verständlich.

Nun wurde aber bereits festgestellt, daB Optimierungsregeln in der Realität nicht beobachtbar sind. Die effiziente schrittweise Ausbeutung der Zensiten kann daher kaum als realistische Perspektive politischen Handelns gewertet werden ${ }^{3}$ ). Offenbar existiert eine ganze Reihe von politischen Restriktionen, die ein einfaches Maximierungskalkül, ganz unabhängig von der Spezifikation der Zielfunktion, unmöglich machen. Die folgenden Abschnitte zeigen einige Eigenheiten des politisch-ökonomischen Willensbildungsprozesses, welche die Reformergebnisse weit mehr beeinflussen, als es das Leviathan-Mode11, das nur ökonomische Beschränkungen kennt, suggeriert ${ }^{4}$ ).

1) So stellt Neumark die Frage: "Was sind die Motive und Ziele von Steuererhöhungen?" Neumark (1982), S. 156. Die Antwort bleibt offen, weil nicht belegt werden kann, ob spezifische Steuererhöhungen nur zur Aufkommenssteigerung oder aber zur Umstrukturierung des Steuersystems dienen. Vgl. Neumark (1982), S. $156 \mathrm{ff}$.

2) Die Notwendigkeit, aber auch die Probleme der Kontrolle zusätzlicher staatlicher Einnahmen, die im Zuge von Steuerreformen erzielt werden, wird in jüngster Vergangenheit immer mehr betont. $\mathrm{Vgl}$. Z.B. die Beiträge zum Deutschen Steuerzahlerkongress (1983).

3) Vgl. Z.B. auch Folkers (1983b), S. 203

4) $\mathrm{Vgl}$. Forte/Peacock (1981), S. 17: [...] the strategies of political parties in capturing votes may have become more complex than the median voter theorem suggest." Vgl. auch Neck (1983), S. 160 
VII.1.5.3. Mehrheitswahlsysteme und wachsender Handlungsspielraum der Politiker

In diesem Abschnitt soll untersucht werden, warum trotz eines funktionierenden Parteienwettbewerbs überhöhte Steuereinnahmen und unsystematische steuerstrukturen entstehen können. ( $\mathrm{Vgl}$. Abb. 3, Pfeil 1). Dabei wird von der Möglichkeit der Kreditfinanzierung abstrahiert und entsprechend den Trends aus Tab. 1 und Tab. 2 in Abschnitt VII.1.3.1. unterstellt, daß wachsende Budgets durch steigende Steueraufkommen finanziert werden.

Zur Illustration des Sachverhaltes dient ein politisch-ökonomisches Modell mit drei Wählern bzw. Steuerzahlern ( $A, B$ und $C$ ), sowie zwei Parteien (I und II). Jede Stimme wird gleich gewichtet. Die Regierung wird von derjenigen Partei gestellt, die bei den Wahlen die meisten Stimmen erhält. Ziel der Parteien ist es, den Anteil des Steueraufkommens, der für eigene Zwecke genützt werden kann, zu maximieren. Das Ziel kann allerdings nur von der. Partei realisiert werden, welche die zu Beginn jeder Legislaturperiode stattfindenden Neuwahlen gewinnt und damit die Regierung übernimmt. Bei der Aufstellung der Parteiprogramme sind die Parteifunktionäre über die Präferenzen der Wähler voll informiert. Die Wähler ihrerseits kennen die Parteiprogramme und geben ihre Stimme der Partei, die ihnen die größten Vorteile verspricht. Da die wahlberechtigten Bürger nur dann eine Partei wiederwählen, wenn diese ihr angekündigtes Programm auch tatsächlich durchführt, sind die Parteien faktisch an ihre Programme gebunden und betrügen nicht. Ferner sei davon ausgegangen, daß ein Parteiprogramm, das zum Wahlsieg führte, von der entsprechenden Partei bei der folgenden Neuwahl beibehalten wird.

Schon in diesem einfachen Modell mit zyklischen Mehrheiten läßt

1) Vgl. Brennan/Buchanan (1980), S. 20 ff. oder Brennan (1981), S. 131. Die Problematik bleibt auch in komplexeren Mehrheitswahlmodellen mit mehreren Parteien, multidimensionalen Entscheidungen oder loyalen Wählern bestehen. Vgi. Boadway/Wildasin (1984), S. $143 \mathrm{ff}$. 
sich zeigen, daß demokratische Wahlen i.d.R. kein optimales $\mathrm{Ni-}$ veau öffentlicher Güter garantieren, bzw. die Möglichkeit zur Ausbeutung der Steuerzahler offen lassen ${ }^{1)}$. Um die Problematik demokratisch legitimierter Steuerreformen deutlich herauszustellen, wird von Manipulationen auf der Ausgabenseite des Staatshaushaltes abstrahiert. Die Regierung möge daher in jeder Periode ein bestimmtes Bündel öffentlicher Güter bereitstellen, das den drei Wählern jeweils gleich hohe Nutzenniveaus garantiert und insgesamt 300 Geldeinheiten kosten möge. Die monetären Aquivalente dieser Nutzenströme lassen sich in einem pay-off-Vektor darstellen, wobei die in Geldeinheiten ausgedrückten Nutzen der Wähler immer in der Reihenfolge $(A, B, C)$ angegeben werden. Der Nutzen aus den öffentlichen Gütern und Dienstleistungen für $A$, $B$ und $C$ kann im vorliegenden Fall z.B. durch den pay-off-Vektor $(200,200,200)$ repräsentiert werden. Für A, B und C ergeben sich die monetären Aquivalente der Nettonutzen aus dem Gesamtbudget durch die Subtraktion ihrer jeweiligen Steuerzahlungen vom Nutzengewinn aus der staatlichen Leistungserstellung.

Im Zweiparteienmodell kann z.B. Partei II eine Struktur der Steuerzahlungen vorschlagen, mit der sie die Wahlen gewinnt, wenn Partei I ein politisch verbindliches Finanzierungsprogramm zuerst preisgeben muß. Gibt Partei I eine Steuerstruktur bekannt, welche die drei Wähler in gleicher Weise belastet, z.B. mit $(100,100,100)$ und daher allen einen gleich hohen positiven Nettonutzen verspricht, $(100,100,100)$, so kann Partei II ein anderes Programm aufstellen, das $A$ und $B$ geringere Steuerzahlungen abverlangt, während $C$ zusätzlich Steuern zahlen muß z.B. (99, 99, 102). Partei II wird die Wahl mit den Stimmen von $A$ und $B$ gewinnen. Wähler $C$ wird jedoch selbst bei geringen Nutzeneinbußen seine Stimme Partei I geben. Aus diesem Grunde wäre es für Partei II überflussig, irgendwelche Nettogewinne für C vorzusehen. Statt dessen könnten Wähler C Steuern in Höhe von maximal 200 Geldeinheiten auferlegt werden. In diesem Fall würde die statliche Bereitstellung von Gütern und Dienstleistungen für C kaum Nutzen abwerfen; die gesamte Konsumentenrente würde ihm entzogen. Der Steuergewinn von 98 Geldeinhei- 
ten stellt den finanziellen Handlungsspielraum der Regierungspartei dar ${ }^{1)}$, der z.B. für Umverteilungsmaßnahmen zugunsten der eigenen Parteimitglieder genutzt werden kann, ohne daB weitere Stimmenverluste zu befürchten wären ${ }^{2}$ ).

In einer dynamischen Betrachtungsweise würde dieses Modell zu einer weiteren Ausbeutung der Wähler führen. Unterstellt man beispielsweise, daß das von Partei II realisierte Regierungsprogramm gleichzeitig auch das Wahlprogramm für die folgende Legislaturperiode darstellt, so kann Partei I durch Reformulierung ihres ursprünglichen Programms die nächsten Wahlen gewinnen und gleichzeitig weitere Ressourcen für eigene Parteimitglieder absorbieren. Das neue Programm müßte für Wähler $C$, der von Partei II völlig ausgebeutet wurde, eine etwas verminderte Steuerbelastung vorsehen, z.B. 199 Geldeinheiten (GE). Ferner könnte z.B. A weniger belastet werden als gegenwärtig von Partei II z.B. mit 98 GE, während B maximal besteuert würde, also mit $200 \mathrm{GE}$. Die darauf folgende Wahl könnte wiederum von Partei II gewonnen werden, indem sie Wähler A 200 GE abverlangt, während C etwas weniger zahlen müBte als vorher, Z.B. $198 \mathrm{GE}$ und B eine geringfügig verminderte Zahllast in Aussicht gestellt wird, z.B. 199 GE. In allen weiteren Folgeperioden werden sich die Parteien durch entsprechende Umstrukturierung der Steuerlast in der Regierungsverantwortung abwechseln, wobei sich die Summe der Nettonutzen aus den jeweiligen Budgets auf einem äußerst niedrigen Niveau einpendeln wird. Die nächste Wahl kann z.B. mit monetär bewerteten Nettonutzen von $(1,2,0)$ gewonnen werden.

1) Vgl. zu diesem Ergebnis Brennan/Buchanan (1980), S. 21

2) Theoretisch wären auch Nettonutzenverluste denkbar. Dies würde jedoch bedeuten, daß die Steuerbelastung für den benachteiligten Wähler so hoch wäre, daß er gänzlich auf Staatsaktivitäten verzichten und die Anarchie befürworten würde. In Analogie zum Leviathan-Modell sei hier und im folgenden unterstelit, daß es keine Regierung riskieren wird, eine Bevölkerungsgruppe derart auszubeuten, daß diese gegen den Staat mobil macht. Ein Nettonutzen von Null scheint daher eine begruindete Grenze für die Ausbeutung einzelner Wählergruppen zu sein. 
Bei der vorliegenden Modellkonzeption muß mit permanent wechselnden Mehrheiten gerechnet werden, so daß ein stabiles politisches Gleichgewicht kaum erreicht wird. Die Summe der payoffs bleibt dabei gleich, lediglich die Verteilung auf die Wăhlergruppen ändert sich. NutznieBer dieses Systems sind die Mitglieder der beiden Parteien; die Wähler dagegen erhalten in wechselnder Folge das jeweilige Nettonutzenminimum, das den Wahlsieg sichert.

Obwohl das einfache Medianwählermodell kein adäquater Spiegel der Realität sein kann, demonstriert es doch die prinzipiellen Möglichkeiten der Parteien, finanzielle Handlungsspielräume für eigene Zwecke zu schaffen. Eine realistische Modellversion müßte allerdings einige zusätzliche Restriktionen bei der Aufstellung von Parteiprogrammen beachten ${ }^{1)}$.

Zyklische Mehrheiten mit einer solch drastischen Umverteilung bzw. Ausbeutung der Zensiten wären in der Realti ät kaum durchsetzbar, weil der gröBte Teil der staatlichen Ausgaben und Einnahmen gesetzlich fixiert ist und deshalb kurzfristig nicht geändert werden kann. Die hohen Gewinne der Parteimitglieder würden Masseneintritte der Wähler und als Reaktion der Parteien Zutrittsverbote erwarten lassen - beide Indizien einer effizienten Wählerausbeutung sind in westlichen Demokratien nicht zu beobachten. Die rigorose Ausbeutung der Steuerzahler hätte sehr wahrscheinlich auch zur Folge, daß neue Parteien gegründet würden, die ihrerseits Steuergewinne anstreben. Oder aber es etablieren sich Parteien, die eine sehr viel geringere Steuerbelastung versprechen. Darüber hinaus bieten die bereits erwähnten institutionellen Restriktionen, aber auch die bekannten Bürgerproteste in den USA, einen ausreichenden Schutz vor den schlimmsten Auswüchsen des Leviathan. Es erscheint somit gerechtfertigt, grundsätzlich nur von inkrementalistischen Umverteilungswirkungen neuer Budgets auszugehen ${ }^{2}$ ).

1) Vgl. dazu die Abschnitte VII.1.1. - VII.1.3.

2) Vgl. zu einem ähnlichen Ergebnis Folkers (1983b), S. $203 \mathrm{ff}$. 
Bei einer schrittweisen Ausbeutung wäre zu erwarten, daß Partei I statt eines pay-off-Vektors von $(101,101,0)$ ein Wahlprogramm anbietet, das pay-offs von (101, 101,97) aufweist und lediglich eine Verrechnungseinheit für eigene Zwecke absorbiert. Partei I könnte dann die nächsten Wahlen mit $(102,98,98)$ gewinnen. Dieses Verfahren zögert den Zeitpunkt der maximalen Ausbeutung hinaus und 1 iefert somit einen Erklärungsansatz dafür, daß die Regierungsparteien sukzessiv und kaum spürbar über viele Perioden hinweg einen stets wachsenden Anteil des Budgets für eigene Zwecke miBbrauchen können ${ }^{1)}$. Das Ausmaß dieses MiBbrauchs hängt in diesem Modell vor allem vom ersten Wahlprogramm von Partei I ab und erreicht sein Maximum bei Budgets mit pay-offs von $(0,1,2)$, wobei sich die Reihenfolge von Periode zu Periode ändern kann. Ein stabiles politisches Gleichgewicht würde in diesem Falle nicht erreicht, weil sich die Parteien durch jeweils variierende Programme in der Regierungsverantwortung abwechseln werden ${ }^{2)}$. Die vorliegenden Modellergebnisse sollten allerdings nicht als einzig mögliche Lösungen derartiger dynamischer Wahlprozesse verstanden werden ${ }^{3)}$. Modifiziert man die diesem Modell zugrundeliegenden Annahmen, in dem man z.B. ein aktiveres Wählerverhalten unterstellt, Informationsmängel auf Seiten aller Beteiligten zuläßt u.s.w., so können sich auch die Modellergebnisse ändern. Bei einer entsprechend modifizierten Modellinterpretation können auch politisch stabile Gleichgewichte abgeleitet werden.

Die Chancen, immer mehr Budgetmittel für eigene Interessen zu absorbieren, nehmen mit wachsenden Budgets weiter zu. Es liegt

1) Diese Grundthese wird durch einen alternativen Erklärungsansatz bei Folkers (1983b), S. $203 \mathrm{ff}$. tendenziell bestätigt.

2) Vgl. zur potentiellen Instabilität des politischen Systems bei einer ähnlichen Modellkonzeption Downs (1957), S. $55 \mathrm{ff}$.

3) Zentrales Anliegen dieses Abschnittes war es nicht, den Nachwe is zu führen, daß die Ausbeutung des Steuerzahlers in demokratischen Staatswesen zwangsläufig mit einer politischen Instabilität einhergehen muß, sondern nur, daß auch in Demokratien eine Ausbeutung der Zensiten möglich ist. 
daher im Interesse der politischen Entscheidungsträger, sowohl bei gegebenem Budgetniveau ein Maximum an Ressourcen zu absorbieren als auch den Budgetumfang selbst jährlich zu erhöhen.

VII.1.5.4. Der Parteienwettbewerb und die politisch opportune Umverteilung von Steuerlasten

Die vorliegende positive Analyse demokratischer Steuerreformprozesse demonstriert einerseits die Gefahr einer sukzessiven Ausbeutung aller Steuerzahler und liefert andererseits eine Erklärung für die zunehmende Diskrepanz zwischen Wählerwünschen und den Ergebnissen des finanzwirtschaftlichen Willensbildungsprozesses, der insbesondere durch wahltaktische Manipulationen in bezug auf die Höhe der Steuerlasten einzelner Zensiten gekennzeichnet ist.

Eine erfolgversprechende Reformstrategie der Regierung besteht somit darin, Steuererleichterungen für spezifische, relativ kleine Gruppen zu schaffen ${ }^{1)}$. Dies kann in Form von Transfers, Steuersatzsenkungen, Steuertarifänderungen oder durch sonstige begünstigende Korrekturen am bestehenden Steuersystem geschehen. Daraus werden sich für die Regierung bei den nächsten Wahlen Stimmen ergeben, weil die einzelnen Gruppen fühlbare Steuererleichterungen erhalten, die sie der amtierenden Partei verdanken ${ }^{2)}$. Unter der Voraussetzung eines konstanten Steueraufkommens müssen diese Steuerausfälle jedoch durch höhere Steuern bei anderen Bevölkerungsgruppen kompensiert werden. Um den Widerstand gegen die Maßnahmen zu reduzieren, muß die Regierung versuchen, die Nachteile (Steuererhöhungen) auf möglichst viele unorganisierte Wähler zu verteilen ${ }^{3)}$.

1) Vgl. Borcherding (1977), S. $58 \mathrm{f}$.

2) $\mathrm{Vgl}$. Miner/Chalice (1978), S. 63

3) Vgl. Folkers (1983b), S. 196, Hettich/Winer (1984), S. 71 formulieren diese These wie folgt: "The government will use available opportunities to lower the effective tax-prices of voters most likely to offer political opposition." 
Deren Steuerlast wird kaum merklich erhöht, so daß das Risiko von Stimmenverlusten gemindert wird. Diese oberlegungen machen erneut auf die Bedeutung der Informationsproblematik aufmerksam. ( $\mathrm{Vgl}$. Abb. 3, Pfeil 7). Je mehr es der Regierung gelingt, das Ausmaß der Umverteilung zu bagatellisieren, und je höher die Informationskosten für die Benachteiligten sind, um so wahrscheinlicher wird der Erfolg einer unmerklichen inkrementalistischen Umverteilungsreform der Regierung ${ }^{1)}$. Systematische Entscheidungen werden auch dadurch erschwert, da $B$ die Bürger am Wahltag nicht nur über ein Projekt oder eine Finanzierungsform entscheiden, sondern über komplette Parteiprogramme, die unzählige Absichtserklärungen beinhalten. Diese "sachliche Undifferenziertheit der Wahlen"2) ermöglicht der siegreichen Partei einen erheblichen monopolistischen Handlungsspielraum ${ }^{3}$ ) in Einzelfragen, die die Wähler bei isolierter Wahl anders entschieden hätten ${ }^{4}$ ).

Informationsdefizite sind jedoch nicht nur auf die gezielte Nachrichtenpolitik der Regierung zurückzuführen, sondern auch auf den rationalen Informationsverzicht ${ }^{5}$ ) seitens der Wähler, die die Kosten zusätzlicher Informationen gegen die daraus erwachsenden Nutzen abwägen. Aufgrund lückenhafter Kenntnisse werden die Steverzahlungen bzw. die Kosten für öffentliche Dienstleistungen möglicherweise unterschätzt und eine zu hohe

1) $\mathrm{Vgl}$. Folkers (1983b), S. $197 \mathrm{ff}$.

2) Roppel (1982), S. 189 und vgl. Folkers (1983b), S. 201

3) Dies gilt insbesondere kurz nach den Wahlen. Je näher der nächste Wahltermin rückt, umso eher wird die Regierung durch Verbesserung der wirtschaftlichen Lage ihre Wiederwahlchancen zu erhöhen trachten. Vgl. zur Theorie der Konjunkturzyklen Frey (1978), S. 205 f.

4) Eine Budgetuiberexpansion ist aber auch dann denkbar, wenn Entscheidungen über unabhängige Ausgabenpläne zeitlich versetzt erfolgen. $\mathrm{Vgl}$. dazu Buchanan/Flowers (1980), S. 173 ff. oder Wildavsky (1980), S. 63.

5) $\mathrm{Vgl}$. zum Problem der Informationskosten bei der Entscheidungsfindung Downs (1957), S. $207 \mathrm{ff}$. 
Nachfrage der Wähler erzeugt. Diese sogenannte Steuerillusion ${ }^{1}$ ) wird insbesondere dann eintreten, wenn Steuern unmerklich erhoben werden, wie es $z . B$. bei indirekten steuern der Fall ist. Dem Trend zu überhöhten Budgets könnten mit politischen Restriktionen, durch eine verbesserte Informationspolitik der opposition, durch die gleichzeitige Veröffentlichung von Programmen anderer Parteien und durch ähnliche Maßnahmen Grenzen gesetzt werden.

VII.1.5.5. Bürokratietheorie und wachsende Budgets

Möglichkeiten zur Schaffung finanzieller Handlungsspielräume bestehen nicht nur auf politischer Ebene. Sie finden sich ebenso in der Bürokratie, die ihre Eigeninteressen wegen ihrer Monopolstellung bis zu einem gewissen Grade realisieren kann ${ }^{2}$. Faktisch trägt die Bürokratie in vielen Bereichen zur Problemformulierung oder -lösung bei, weil nur sie die Informationen und das Fachwissen besitzt, um die "Machbarkeit" politischer Vorstellungen zu beurteilen ${ }^{3)}$. Sie kann daher Vorgaben der exekutierenden Politiker selektieren, modifizieren oder zurückweisen. Die Unabhängigkeit von Wahlen und die Unkündbarkeit der meisten Bürokraten festigt ihre Position zusätzlich.

Anders als in einer institutionell leeren Theorie wird hier unterstellt, daß die Bürokraten ihren monopolistischen Handlungsspielraum für das Streben nach administrativer Macht und Sicherheit einsetzen ${ }^{4}$, die sich z.B. in der GröBe ihrer Büros und der Wachstumsrate ihres Budgets ausdrücken.

1) Vgl. Z.B. Buchanan (1977b), S. 11, Folkers (1983b), S. 201 und zur Gegenposition Musgrave (1981a), S. $98 \mathrm{ff}$.

2) Restringiert wird die Bürokratie dort, wo sie die Position der Regierung gefährdet. "It is only when the recommendations of the bureaucracy present obvious possibility of damaging vote positions they will be rigorously checked by government", Bartlett (1973), S. 63

3) Vgl. dazu Z.B. Roppel (1979), S. 17 oder Boskin (1978), S. 25

4) Vgl. z.B. Bartlett (1973), S. $21 \mathrm{f}$. 
Das Streben nach einem angemessenen Wachstum des Büros bzw. nach Budgetmaximierung ${ }^{1)}$ wäre daher auch eine empirisch gehaltvolle Verhaltenshypothese ${ }^{2}$ ).

Um die Maximierungsstrategie der Bürokraten herausarbeiten zu können, wird im folgenden ein ideales Wahlmodell unterstellt, in welchem die Partei, die die Wahlen gewinnen will, sich möglichst nahe an das vom Medianwähler gewünschte Budget herantasten muB. Unter den Voraussetzungen einer vorgegebenen Steuerstruktur, vollkommener Information der politischen Entscheidungsträger, beliebiger Teilbarkeit der Güterbündel, gleichzeitiger Bekanntgabe der Parteiprogramme und eingipfeliger Präferenzen werden die Parteien jenes Budget vorschlagen, das sich der Medianwähler wünscht. Dieses Referenzmodell bringt Wählerpräferenzen und Parteiprogramme in Einklang und führt zu einem politischen Gleichgewicht, das durchaus pareto-optimal sein kann ${ }^{3}$ ). Medianwähler und politische parteien können nun als Einheit betrachtet werden, die einer budgetmaximierenden Bürokratie gegenüberstehen. In dieser Situation werden die Parteien von der Bürokratie verlangen, daß ihre Parteiprogramme, die gleichzeitig den Wünschen des Medianwählers entsprechen, erfuilt werden.

Die Eigenart bürokratischer Leistungserstellung besteht aber darin, daß die Politiker bzw. die Wähler, nicht eine beliebige Budgetgröße, sondern lediglich zwischen zwei Optionen wählen ${ }^{4}$ )

1) Vgl. Niskanen (1971), S. $30 \mathrm{ff}$. und S. 42

2) Vgl. dazu Borcherding (1977), S. 57 ff., Buchanan (1977), S. 4 ff. oder Roppel (1979), S. 86

3) Vgl. zu dieser Modellkonzeption MacKay/Weaver (1978), S. 146 ff. oder Boadway/Wildas in (1984), S. $143 \mathrm{ff}$.

4) Vgl. Romer/Rosenthal (1979). Die folgende Analyse beruht weitgehend auf dem dort vorgestellten Modell der Monopolbürokratie. 
können. Das Machtpotential der Bürokratie, das es ihr erlaubt, als optionsfixierer aufzutreten, beruht erstens auf ihrem Informations- und Angebotsmonopol, das sie bei gegebener Nachfrage zur Erweiterung ihrer Budgets nutzen kann ${ }^{1}$. Zweitens werden die Budgetverhandlungen zwischen Politikern und Bürokraten nicht durch Grenznutzen-Grenzkostenvergleiche einzelner Leistungseinheiten gekennzeichnet, sondern durch Vereinbarungen über ganze Leistungspakete ${ }^{2}$ ). Dies erhöht zum einen die Informationskosten und führt dazu, daß der Medianwähler auch solchen Gütern und Dienstleistungen zustimmt, die er bei Einzelabstimmungen ablehnen würde. Ferner wird unterstellt, daß die Bürokraten die Präferenzen der Wähler für die von der öffentlichen Hand bereitgestellten Güterbündel bei alternatigen Finanzierungsstrukturen bzw. Budgetniveaus kennen ${ }^{3)}$. Es geht daher um die Frage, inwieweit es den Bürokraten gelingt, höhere Budgets durchzusetzen als sie der Medianwähler in seinem optimum zu realisieren wünscht.

Im einfachsten Fall werden die Bürokraten bei Einführung neuer Dienstleistungsprogramme die Politiker vor eine Alles-oderNichts-Alternative stellen $\left.{ }^{4}\right)$. (Vgl. Abb. 3, Pfeil 2) Das maximale Budget, das vom Medianwähler akzeptiert würde, muß gegenuber der Situation ohne Budgets wenigstens eine geringe Nutzenverbesserung aufweisen.

Ein solches Budget würde nahezu die gesamte Konsumentenrente des Medianwählers aus der Leistungsbereitstellung abschöpfen; d.h. die Bürokratie würde ähnlich dem Niskanen-Modell die maximale Zahlungsbereitschaft der Politiker bzw. des Medianwählers absorbieren ${ }^{5}$ ).

1) Vgl. Roppel (1979), S. 103

2) Vgl. Roppel (1979), S. $106 \mathrm{ff}$.

3) Vgl. Romer/Rosenthal (1979), S. 564

4) Vgl. MacKay/Weaver (1978), S. 147

5) Vgl. Roppel (1979), S. 107. Er geht bei den Verhandlungen von einem bilateralen Monopol aus. 
Die maximale Ausbeutungsstrategiewird bei realistischer Betrachtungsweise jedochnicht zu erwarten sein, weil die Politiker aus Furcht vor einer Wahlniederlage ihre hierarchische Vorrangstellung gegenüber der Bürokratie einsetzen werden (Pfeil 9). So ist es denkbar, daß den Bürokraten anfangs nur ein kleines Budget zugestanden wird, weil die Politiker zunächst die Wählerreaktionen testen wollen. Aber auch in diesem Falle besteht die Gefahr überhöhter Budgets. Wenn beispielsweise das neue Programm bei den Bürgern positiv aufgenommen wurde, können die Bürokraten be $i$ den nächsten Verhandlungen wiederum ein Maximalbudget zur Disposition stellen, das einen höheren Nettonutzen für den Medianwähler gewährleistet als das ursprüngliche Minimalbudget, ("revision expenditure")"). Da der Medianwähler durch das gröBere Budget besser gestellt würde, wird auch in diesem Fall ein zu großes Budget akzeptiert werden.

In einer Ausgangssituation, in der das ursprüngliche Budget gröBer als das 0ptimalbudget war, wird es für die Bürokraten erheblich schwieriger werden, eine Budgetausweitung zu erwirken. In solchen Fällen werden sie in der Regel nur inkrementalistische Budgeterhöhungen durchsetzen können ${ }^{2}$ ). Je schwieriger und komplexer die Steuergesetzgebung wird ${ }^{3)}$, um so notwendiger wird eine besondere administrative Auslegung und um so mehr wächst die Bedeutung der Bürokratie 4 ). Die Steuerbürokratie hat daher keinen Anreiz, ein einfaches, normkonsistentes Steuersystem zu konzipieren (Pfeil 11).

1) Vgl. Romer/Rosenthal (1979), S. 564

2) Vgl. Davis/Dempster/Wildavsky (1979), S. $372 \mathrm{ff}$.

3) Diese Entwicklung wird von vielen Fachleuten (erfolalos) beklagt. Vgl. Z.B. Rose (1983), S. 100 , Schneider (1983), S. 64 ff., Schmölders/Schelle (1978), S. 21, Neumark (1982), S. 156 oder Pechman (1977), S. 46 f. Immerhin haben diese Erkenntnisse mittlerweile zu Maßnahmen der Entbürokratisierung und zum Entwurf des Steuerbereinigungsgesetzes 1985 geführt. Ironischerweise müssen aber zur Prüfung der Maßnahmen zunächst Kommissionen gebildet werden. $\mathrm{Vg} 1$. hierzu die Finanznachrichten des Bundesministeriums der Finanzen $14(1984)$, S. 2 und das Bulletin des Presseund Informationsamtes der Bundesregierung 133 (1983), S. 1211

4) Einige interessante empirische Belege für diese These finden sich bei Riedel (1982), Radian (1979) oder Wassermayer (1983) 
VII.2. Die Rolle der Steuerzahler im SteuerreformprozeB

Der politisch-ökonomische Rahmen des Steuerreformmodells (Abb. 3) wird im folgenden durch die Berücksichtigung der Einflußmöglichkeiten der Steuerzahler auf den steuerpolitischen Willensbild gsprozeß vervollständigt (vgl. Abb. 3 , Pfeile 3 , $4,5,6$ und ). Das Fehlen dieser Beziehungen ist ein Mangel in den OT- und Leviathan-Modellen, die übereinstimmend keine politischen Reaktionen der Steuerzahler auf Anderungen des steuerlichen Instrumenteneinsatzes berücksichtigen ${ }^{1)}$.

Insbesondere bei Brennan/Buchanan ${ }^{2}$ ) wird der Eindruck erweckt, als gäbe es ausschließlich wehrlose Opfer staatlicher Steuerwillkür, die sich nur durch das Einstellen sämtlicher Wirtschaftsaktivitäten oder durch eine Verfassung dem fiskalischen Zugriff entziehen könnten. Diese Vorstellung muß in einer positiven Theorie des Steuerzahlerverhaltens korrigiert werden.

VII.2.1. Das Konsumentenverhalten

Den Konsumenten wird nach wie vor Nutzenmaximierung unterstellt. Dies impliz rt, daß die Steuerzahler primär ihre Gesamtsteuerlast zu mini ieren suchen, ohne Rücksicht auf irgendwelche übergeordneten (Effizienz- oder Gerechtigkeits-) Ziele der Besteue-
1) Vgl. Kap. II und IV
2) Vgl. Brennan/Buchanan (1980) 
rung ${ }^{1)}$. Sie werden also durchaus eine höhere Mehrbelastung in Kauf nehmen, wenn sie dadurch ihre Gesamtbelastung (Zahllast + Mehrbelastung) mindern können ${ }^{2)}$. Obwohl die These der Gesamtlastminimierung durchaus kritikfähig ist, stellt sie jedoch eine wesentlich realistischere Verhaltenshypothese dar, als die implizite Anpassungshypothese der oben diskutierten Leviathan-oder OT-Modelle.

Ferner muß eine positive Steuerreformtheorie davon ausgehen, daß sich die Steuerzahler aktiv in den politischen Reformprozeß einschalten ${ }^{3)}$. Dazu stehen ihnen grundsätzlich zwei

1) Vgl. zu dieser Verhaltenhypothese Alt (1983), S. $182 \mathrm{ff}$. oder Folkers (1984), S. 2. Stützel druickt es pointiert wie folgt aus: "Steuerzahler sind geborene Steuervermeider", Stuitzel (1982). Als empirischen Beleg dieser Hypothese können die Steuerrevolten in den USA gewertet werden. Als Ursachen für die Revolten werden immer wieder Steuerlastsenkungen angeführt. Vgl. Z.B. Oakland (1981), S. 33, Rabushka/Ryan (1982), S. 7. Gegner der Proposition 13 waren interessanterweise Politiker und Lehrer, weil die Schulausgaben vorwiegend über die pronerty tax finanziert wurden. $\mathrm{Vgl}$. auch Folkers (1983a)

2) $\mathrm{Vgl}$. Folkers (1983b), S. 191. Auch hier sei wieder der Hinweis erlaubt, daß die Zielsetzung der Konsumenten allein noch nichts über den Grad der Zielerreichung aussagt. Inwieweit die Ziele einzelner Steuerreformteilnehmer realisiert werden können, hängt vielmehr von den gegebenen Restriktionen ab. In der Realität sind daher die individuellen Zielsetzungen von Regierung, Konsumenten oder Interessengruppen außerordentlich schwer zu identifizieren, weil ihr Verhalten durch vielfältige ökonomische und politische Restriktionen erheblich beeinträchtigt und modifiziert wird. So könnte man dem einzelnen Steuerzahler auch unterstellen, daß er die Steuervorteile nicht nur wegen des finanziellen Gewinns anstrebt, sondern auch deshalb, weil er sich tatsächlich durch das aktuelle Steuersystem ungerecht behandelt fühlt. Vgl. Keene (1982), S. $371 \mathrm{ff}$. oder Musgrave (1981b), S. 30. Diese Zielvariante verstärkt jedoch nur die hier unterstel1te Grundhaltung der Individuen, so daß sie im folgenden nicht weiter erwähnt werden muß.

3) Vgl. Folkers (1983b), S. 190. Obwohl das Eigeninteresse der Steuerzahler das zentrale Motiv für ihre Bereitschaft zum aktiven Eingreifen in den politischen Prozeß charakterisiert, erklärt es allein noch nicht, warum verschiedene Wirtschaftssubjekte zu bestimmten Zeiten besonders aktiv werden. Das Engagement bestimmter Steuerzahler während der Steuerrevolte in den USA wird deshalb mit bestimmten "Katalysatoren", wie der Höhe der Steuerlast und der Fristigkeit der Steuerlaständerung in Verbindung gebracht, deren Bedeutung auch empirisch nachzuweisen versucht wurde. Vgl. Ste in, Hamm, Freeman (1983), S. $187 \mathrm{ff}$. 
Wege offen: Erstens können sie bei Wahlen derjenigen Partei ihre Stimme geben, von der sie glauben, daß sie ihre Interessen möglichst weitgehend verfolgt (vgl. Abb. 3, Pfeil 6); zweitens haben sie die Möglichkeit, ihre Forderungen durch Interessengruppen, Verbände oder Bürgerinitiativen in politischen Entscheidungsprozessen geltend zu machen $\left.{ }^{1}\right)$. (Vgl. Abb. 3, Pfeil 7) Diese Alternative zur Wahlbeeinflussung hat den Vorteil, daß die gruppenspezifischen Steuerreformwünsche ohne Abstriche und sehr gezielt zur Geltung kommen können ${ }^{2}$ ). Weiterhin steht es dem Bürger offen, auch mehreren Gruppierungen beizutreten (oder sie zu gründen), so daB er eine Interessenvertretung auswählen kann, die seine Wünsche möglichst umfassend repräsentiert. Da Forderungen eines einzelnen Steuerzahlers normalerweise keine Bedeutung für politische Entscheidungsabläufe haben, wird im folgenden grundsätzlich davon ausgegangen, daß sich die Steuerzahler von Interessengruppen vertreten lassen.

\section{VII.2.2. Das Produzentenverhalten}

Die Produzenten seien Gewinnmaximierer. Ebenso wie die Konsumenten können sie aktiv in den politischen ProzeB eingreifen. Da wirtschaftliche Macht bei der Stimmabgabe ohne Gewicht ist,

1) Häufig wird vermutet, daß der wesentliche Einfluß auf die detaillierte Ausgestaltung der Steuergesetzgebung nicht von Wahlen herrührt, sondern aus der Intervention von Interessengruppen. Vgl. z.B. Bartlett (1973), S. 64. Ein interessantes Beispiel liefert Reese (1980), S. 29: "As a senator and then as president, Johnson opposed tax reform because it had become identified with cutting the oil deplation allowance, which was important to both, Texans and presidential campa in financiers". Ahnliche Zusammenhänge zwischen finanzkräftigen Interessengruppen und Politikern werden auch in der Bundesrepublik Deutschland im Zusammenhang mit dem Fall Flick untersucht.

2) Vgl. Folkers (1983b), S. $198 \mathrm{f}$. 
stellen die Formierung relativ kleiner, wohlorganisierter Interessengruppen ${ }^{\uparrow}$ ) und eine gezielte Informationspolitik ihre wichtigsten Instrumente zur Beeinflussung der politischen Akteure dar (vgl. Abb. 3, Pfeile 4 und 5). Von der Beeinflussung der Konsumenten z.B. durch Werbung wird im folgenden abstrahiert, weil sie für die hier betrachteten Zusammenhänge ohne Bedeutung ist (Pfeil 8 ).

Wenngleich sich die Interessen von Produzenten und Konsumenten in bestimmten Bereichen unterscheiden mögen, haben sie sicherlich auch gleichgerichtete Interessen, die sie durch dieselben Verbände vertreten lassen können, so daß im weiteren Verlauf der Untersuchung auf eine spezifische Produzenten-KonsumentenDifferenzierung verzichtet werden kann, ohne die Gïltigkeit der Ergebnisse zu beeinträchtigen.

\section{VII.2.3. Die Rolle von Interessengruppen im Steuerreformprozeß}

Viele Phänomene des Steuerreformprozesses können nur durch das spezifische Verhalten von Interessengruppen erklärt werden ${ }^{2}$ ). Interessenverbände werden von Bürgern geschaffen und unterhalten, damit sie deren Interessen gegenüber den politischen Entscheidungsträgern vertreten ${ }^{3}$ ).

1) Vgl. Becker (1983), S. 385, 01 son (1968) oder Folkers (1983b). Zum Einfluß von Interessengruppen im politischen Proze $\beta$ siehe z.B. Bernholz/ Breyer (1984), S. $349 \mathrm{ff}$.

2) Dies gilt nicht nur für die Analyse von Steuerreformen. So versucht 01 son (1982) eine Beziehung zwischen gesellschaftlicher Wohlfahrt und dem Organisationsgrad von Interessengruppen zu belegen. Natürlich bedeutet dies nicht, daß das bloße Bestehen von Verbänden bereits einen politischen Einfluß belegt. Vgl. Wagner (1966), S. $161 \mathrm{ff}$.

3) Vgl. 01 son (1982), S. 18. Effekte, die auf unterschiedliche Gruppengrößen zurückzuführen sind, werden im folgenden vernachlässigt. Siehe 0lson (1982), S. 41. Damit bleibt auch unberüicksichtigt, daß der Druck auf ganz spezifische Grupoenvorteile mit zunehmender Verbandsgröße tendenziell abnimmt. $\mathrm{Vgl}$. 01 sen (1982). So läßt sich auch beobachten, daß große Dachverbände regelmäßig sehr viel moderatere Ansprüche erheben als Einzelorganisationen. 
Es darf jedoch nicht erwartet werden, daß die führenden Interessenvertreter lediglich ein verlängerter Arm der Konsumentenbzw. Produzentenwïnsche sind. Obwohl sie bei Wahlen und ihrer Finanzierung (Pfeil 7) bis zu einem gewissen Grade von ihrer Basis abhängig sind, verfolgen sie auch Eigeninteressen, indem sie, wie Bürokraten, ihre Macht und Budgets auszuweiten versuchen. Es wird daher angenommen, daß Verbandsfunktionäre ihren Nutzen dadurch maximieren, daß sie möglichst einflußreiche und ertragreiche Positionen erwerben, unter der Nebenbedingung, daß sie in ihrem Amt bestätigt werden.

Zu diesem Zweck müssen sie ihre Bedeutung durch eine gezielte Informationspolitik gegenüber der Basis herausstellen (Pfeil 5) und sich so einen Handlungsspielraum schaffen. Ihre Existenzberechtigung erhalten sie aus den Bemühungen um politische Vorteile, hier insbesondere um Steuervorteile ${ }^{1}$ für Verbandsmitglieder ${ }^{2}$ ) und $\mathrm{K} 1$ ientel.

Dies kann am Beispiel der Steuersachverständigenberufe verdeutlicht werden. Die Leistungen der Steuerberater werden nachgefragt, weil man annimmt, mit ihrer Hilfe Steuereinsparungen zu erzielen, die über den aufgewendeten Honoraren liegen. Je länger aber ein Steuersystem unverändert bleibt, um so eher werden die Zensiten in der Lage sein, ihre Steuervor-

1) Der Einfluß der Interessengruppen besteht natürlich auch auf der Ausgabenseite. Verbände die relativ geringe Steuersätze durchsetzen können, werden für höhere Staatsausgaben plädieren, weil diese Ausgaben relativ billig angeboten werden. Dieser Zusammenhang würde auch eine Erklärung für das relative Wachstum der Staatstätigkeit liefern. Vgl. Mueller/Murrell (1983)

2) Ein anschaulicher Beleg für diese These findet sich bei Heilmann (1975), S. $289 \mathrm{ff}$. Von 760 an den Bundestag gerichteten Eingaben und Anträgen zur Umsatzsteuerreform, d.h. während der Entstehung des Umsatzsteuergesetzes von 1967 , zielten 504 oder $66,3 \%$ auf eine totale oder partielle Umsatzsteuerbefreiung ab. 
teile selbst wahrzunehmen. Viele Interessengruppen haben daher ein existenzielles Interesse an einem komplizierten steuersystem und an permanenten Steuergesetzänderungen. Hier findet sich eine weitere Erklärung für den permanenten Reformdruck, der nicht von den Zielsetzungen der Steuerzahler, sondern vorrangig von der Motivation der Interessenvertreter ausgeht.

\section{VII.2.4. Individuelle Verteilungsvorteile als Steuerreformziel}

Im Zentrum der individuellen Reformstrategien ${ }^{1)}$ stehen die Erhaltung und Schaffung spezifischer Verteilungsvorteile. (Vgl. Abb. 3, Pfeil 10). Dementsprechend werden Steuerreformen im politischen Alltag meist im Sinne von Russell Lang definiert: "A change in the tax law that I favor"2). Das kann auf vielfältige Weise geschehen; z.B. durch geeignete Steuertarif-und Strukturänderungen, durch die Erosion der Steuerbemessungsgrundlage oder die Realisierung individueller Steuervergünstigungen. Für die einzelnen Interessengruppen sind jedoch nicht alle Bemühungen um steuerliche Verteilungsvorteile gleichermaßen attraktiv ${ }^{3)}$. Die Intensität der Reformbemühungen hängt unter anderem von der Form und der Höhe der erwarteten Steuervorteile ab. Umfassende Reformen, die allgemeine Steuersenkungen zur Folge haben, wie z.B. die Reduktion des Mehrwertsteuersatzes oder die Milderung der nominellen Progression des Einkommensteuertarifs,

1) Shoup (1970), S. 252 spricht von Reformtaktiken, Tullock (1983), S. 2: "The major motive for government income transfer in the modern world and in fact throughout history, is simply that the recipients of the money would like to get it, and they have the political power, or in some cases the luck to inplement the ir desires." $\mathrm{Vgl}$. auch McCormick/Tollison (1981), Kap. 1

2) $\mathrm{Vgl}$. Reese (1980), S. 6

3) Vgl. Folkers (1983b), S. $196 \mathrm{ff}$. 
werden von einzelnen Interessengruppen im laufenden Steuerreformprozeß kaum angestrebt werden. Dafür gibt es mehrere Gründe: zunächst ist festzustellen, daß bei derartigen Steuerreformaktivitäten hohe Kosten anfallen. Denn gerade eine mit allgemeinen Verteilungsvorteilen verbundene Minderung des Steueraufkommens müßte gegen die vitalen Interessen der Regierung und Bürokraten durchgesetzt werden - ein Bestreben, das auch mit Blick auf die empirischen Daten bisher keinen dauerhaften Erfolg hatte ${ }^{1)}$. Dies bedeutet jedoch nicht, daß es keine politisch-ökonomischen Konstellationen geben könnte, welche es den politischen Entscheidungsträgern ratsam erscheinen lassen, allgemeine Steuersenkungen anzustreben ${ }^{2}$. Denkbar wäre eine solche Situation unter der Voraussetzung, daß der Druck der Wählermehrheit aufgrund allgemein sinkender Realeinkommen bei steigender Steuerbelastung so groß wird, daB allgemeine Steuersenkungen mehr Stimmengewinne einbringen als die Vergabe spezifischer Steuervergünstigungen. Derartige Steuerreformbemühungen sollen jedoch im folgenden nicht eingehender untersucht werden.

Darüber hinaus bergen allgemeine Steueraufkommenssenkungen die Gefahr einer verringerten Bereitstellung von öffentlichen Gütern und Dienstleistungen, so daß die Unterstützung der Wählermehrheit nicht gesichert erscheint ${ }^{3)}$. Das Potential allgemeiner Steuertarifermäßigungen oder der Verkürzung der Einkommensteuerbemessungsgrundlage muß deshalb gering eingeschätzt werden. Den hohen Reformkosten der aktiven, organisierten Interessengruppen würden somit nur geringe potentielle Vorteile der eigenen Klientel gegenüberstehen. Daraus resultiert ein verminderter Reformdruck schlagkräftiger Organisationen, der zusätzlich durch das Free-rider-Verhalten der begüstigten Allgemeinheit weiter abgeschwächt wird. Da allgemeine Steuer-

1) Vgl. die Entwicklung der STeuerquote in Tab. 2, Abschnitt VII.1.3.1.

2) Ein Beispiel hierfür wäre die in jüngster Zeit vorgeschlagene Senkung und Vere infachung des Einkommensteuertarifes in den USA.

3) Vgl. zu einigen Erfahrung44en in den USA Folkers (1383a), S. $34 \mathrm{ff}$. 
vergünstigungen den Charakter öffentlicher Güter haben, werden sich die einzelnen Wirtschaftssubjekte kaum veranlaßt sehen, die hohen Reformkosten einzelner Initiatoren mitzutragen. "Tatsächlich resultiert jedoch keine generelle Verminderung des Reformdrucks, sondern eine Verminderung von Reformvorhaben mit generellen Wirkungen; d.h. die Dispersion der einzelnen Steuervergünstigungen wird sich jeweils nur auf relativ kleine Gruppen beziehen, da die Organisations- und dadurch die Vermeidungskosten des Free-rider-Verhaltens hier gering sind"1). Es ist daher zu erwarten, daB von den Betretern der Interessengruppen nur solche Steuerprivilegien angestrebt werden, die speziell ihren Mitgliedern zugutekommen und gleichzeitig ihren Erfolg dokumentieren. Dieses Ergebnis ist mit dem Verhaltensmuster der finanzpolitischen Entscheidungsträger kompatibel, die ihren Wahlerfolg mit Hilfe gruppenspezifischer Steuergeschenke, d.h. einer sukzessiven Steuerlastumverteilung sichern wollen. Aus diesen sich perpetuierenden Reaktionen resultiert die nahezu unübersehbare Vielfalt steuerrechtlicher Ausnahmeregelungen, insbesondere von Steuervergünstigungen (loopholes), die für das $\operatorname{chaos}^{2}$ des aktuellen steuersystems großteils verantwortlich sind und seine effektive Belastungswirkung weitgehend mitbestimmen.

\section{VII.2.5. Die Allokations- und Verteilungswirkungen von Steuer-} vergünstigungen

Im vorangegangenen Abschnitt wurde festgehalten, daß das wesentliche Ziel von Interessengruppen im SteuerreformprozeB darin besteht, möglichst viele und hohe individuelle Verteilungsvorteile bzw. Steuervergünstigungen für ihre Mitglieder zu erreichen. Der wesentliche Grund für diese Absicht ist die Senkung der individuellen Gesamtsteuerlast. Dabei verstellt die einsei-

1) Folkers (1983b), S. 196

2) Vgl. Tipke (1976), S. $293 \mathrm{ff}$. 
tige Verfolgung von Partikularinteressen ${ }^{1)}$ den Blick sowohl für gravierende gesamtwirtschaftliche Allokationsstörungen als auch für die Verteilungsungerechtigkeit. Die folgende Analyse zeigt, daß die Vergabe von Steuervorteilen nach politischer Opportunität in der Regel zu erheblichen Ressourcenverlusten führen wird. Bevor diese Behauptung belegt werden kann, bedarf es zunächst einer Definition "gruppenspezifischer Steuerprivilegien".

\section{VII.2.5.1. Zur Definition individueller Steuerprivilegien}

Zum Zwecke dieser Untersuchung scheint es sinnvoll, eine möglichst umfassende Begriffsbestimmung zu wählen ${ }^{2}$ ). Daher werden im folgenden unter den Begriffen Steuerprivilegien oder Steuervorteilen nicht nur alle Subventionen, Finanzhilfen, Steuervergünstigungen. Transferzahlungen und sonstige direkte und indirekte finanzielle Unterstützungen des States an bestimmte Interessengruppen innerhalb und außerhalb des statichen Sektors subsumiert ${ }^{3}$ ), sondern auch jede Manipulation der Steuersätze, Steuertarife oder Steuerbemessungsgrundlagen zugunsten einzelner Produzenten- und Konsumentengruppen ${ }^{4}$ in der Absicht, deren Steuerlast zu senken. Auch wenn die verschiedenen Steuerprivilegien gleichen Aufkommens keine identischen Allokations- und Verteilungswirkungen erwarten lassen ${ }^{5)}$, führen sie im Sinne dieser sehr weiten Definition zu prinzipiell glei-

1) Shoup (1979), S. 252 spricht von Reformtaktiken.

2) In der Literatur findet sich keine einheitliche Definition. Vgl. Z.B. die unterschiedliche Begriffssystematik in den Ministerien: Siebter Subventionsbericht (1979), S. 4 ff., unter der Verantwortung des Bundesministers der Finanzen und dem Zwischenbericht der Transfer-Enquête-Kommission (1979), S. $36 \mathrm{ff}$., unter der Verantwortung des Bundesministers für Wirtschaft und des Bundesministers für Arbeit und Sozialordnung.

3) Entgegen dem Siebten Subventionsbericht (1979), S. 5, fallen somit auch die Zuwe isungen und Zuschüsse an die Deutsche Bundesbahn und Deutsche Bundespost unter den Subventionsbegriff.

4) Von der folgenden Diskussion werden lediglich jene Transferzahlungen ausgenommen, die zur Sicherung des Existenzminimums dienen. $\mathrm{Vgl}$. auch Kap. IX.

5) $\mathrm{Vgl}$. Feldstein (1980), S. $99 \mathrm{ff}$. 
chen Reaktionen der Wirtschaftssubjekte, so daß die Begriffe im folgenden synonym verwendet werden können.

\section{VII.2.5.2. Die Allokationswirkungen}

Der exakte theoretische Nachweis von Effizienzverlusten aufgrund einer Steuerprivilegvergabe kann nur im konkreten Einzelfall gelingen. Aus der Theorie des Zweitbesten weiB man, daB einzelne Steuervergünstigungen durchaus positive Allokationseffekte haben können, und das selbst dann, wenn sie verteilungspolitisch motiviert sind. Von solchen wohlfahrtssteigernden Zufälligkeiten politisch erkämpfter Steuerprivilegien wird im Partialmodell abstrahiert, auch wenn sie theoretisch nicht auszuschließen sind. Diese Problematik wird in vielen Literaturbeiträgen, insbesondere aber in der aktuellen Subventionsdiskussion weitgehend ignoriert. Häufig werden die aus Subventionen resultierenden Allokationsverluste einfach postuliert, auch wenn die folgenden Argumente zeigen, daB diese Behauptung durchaus gerechtfertigt und belegt werden kann. In der politischen Realität wird die Unsystematik des Willensbildungsprozesses ${ }^{1}$ ) durch viele kleine Reformen und die sukzessive Vergabe von Steuervergünstigungen ohne Berücksichti-

1) "We will also see [...] that the choice among all the possible tax institutions will not be made on the basis of normative considerations but will arise from the political environment this behavior creates". Bartlett (1973), S. 89 
gung gesamtwirtschaftlicher steuerziele offenkundig ${ }^{1)}$. Die unsystematische Vergabe von Subventionen wird vor allem dadurch gefördert, daß z.B. "in der Bundesrepublik nicht nur Gesetze sondern auch einfache Parlamentsbeschlüsse oder Mittelzuweisungen im Haushalt als ausreichende Rechtsgrundlage angesehen werden, ferner der Umstand, daß die Subventionsvergabe teilweise ohne publizierte Verwaltungsvorschriften erfolgt"2).

Im Rahmen der positiven Steuertheorie wird deshalb nur dann von Steuerprivilegien gesprochen, wenn steuerliche Ausnahmetatbestände ohne Berücksichtigung des umfassenden Steuersystems und zur Begünstigung irgendwelcher Gruppen, Sektoren oder wirtschaftlicher Aktivitäten geschaffen werden sollen ${ }^{3)}$. In der gegenwärtigen politischen Subven-

1) So beklagt Surrey (1973), S. $30 \mathrm{ff}$., daß die Einkommensteuergesetzgebung der USA eigentlich aus zwei verschiedenen Steuerstrukturen zusammengesetzt ist. Einmal existiert diese aus "the normative income tax model and its necessary auxiliary provisions. The second structure is that reflecting the Tax Expenditures Budget and contains the provisions carrying out financial assistance set forth in that budget". Die verschiedenen Systeme mit verschiedenen Funktionen machen die Steuergesetzgebung äußerst komplex und erfordern verschiedene Experten, so daß letztlich weder eine effiziente noch eine gerechte Besteuerung erfolgen kann. Vgl. zu dieser Kritik Z.B. auch Steuerreformkommission (1971), S. $241 \mathrm{ff}$. Ganz ähnlich muß die Situation in der Bundesrepublik Deutschland eingeschätzt werden, wenn auch "große" Einkommensteuerreformen ohne Bezug auf das bestehende Transfersystem diskutiert werden.

2) Vgl. Andel (1977), S. 503 oder für die USA: Surrey/Mc Daniel (1980), S. 1431

3) Dieser Hinweis ist deshalb wichtig, weil eine Regierung beispielsweise durch eine Senkung der Verbrauchsteuersätze bei allen freizeitkomplementären Gütern und einer Erhöhung der Steuersätze bei freizeitsubstitutiven Gütern bei gleichem Steueraufkommen eine Wohlfahrtssteigerung erreichen kann. Derartige Steuersatzsenkungen, die unter Berücksichtigung des gesamten Steuersystems vorgenommen werden, um ein Second-BestOptimum zu erreichen, fallen hier nicht unter den Begriff Steuervergünstigungen, weil sie Resultat eines umfassenden Optimierungskalkuils bei vollkommener Information sind. $\mathrm{Vgl}$. Kap. II. Vgl. auch Folkers (1985). 
tionspraxis sind diese Voraussetzungen üblicherweise erfüllt. Die Allokationswirkungen von Steuerprivilegien werden allenfalls noch auf streng komplementären oder substitutiven Märkten mitbetrachtet, sofern verlässliche Informationen überhaupt zur Verfügung stehen und das zur offiziellen Rechtfertigung dienende Allokationsziel auch tatsächlich eine Entscheidungskomponente darstel1 $1 t^{1}$. Geht es dagegen um Steuerverguinstigungen mit distributiver Zielsetzung, so werden ihre Allokationswirkungen erst gar nicht in der politischen Diskussion debattiert. Infolgedessen können Allokationsverbesserungen auch nur rein zufällig entstehen ${ }^{2}$ ).

Doch selbst Steuereingriffe, die zum Zwecke einer effizienteren Allokation eingesetzt werden, sind nur dann unzweifelhaft wohlfahrtserhöhend, wenn sie bei ausreichender Information über Angebots- und Nachfragebeziehungen bzw. unter Berücksichtigung des bisherigen Gesamtsteuersystems eingefügt werden. Ohne Kenntnis der Effekte aufgrund wirtschaftlicher Interdependenzen sollte im allgemeinen von steuerlichen Eingriffen in den marktwirtschaftlichen Allokationsmechanismus abgesehen werden ${ }^{3}$ ). Die Empfehlung beruht auf

1) Ahnliche Bedenken äußert Z.B. Zimmermann, (1977). S. $12 \mathrm{ff}$. Dies gilt insbesondere für Steuervergünstigungen (d.h. für Steuerprivilegien in Form niedrigerer Steuersätze). Vgl. Surrey (1973), S. 33

2) Etwas drastischer verurteilt Issing die gegenwärtige Subventionspraxis. "Dies schließt selbstverständlich nicht aus, daß im Einzelfall Subventionen einen vernünftigen Zweck erfüllen, doch kann mit solch zufälligen 'Treffern' nicht der theoretische Anspruch begründet werden", Issing (1983), S. 56 oder Molitor (1984), S. 117 ff. Einige gravierende Beispiele für unsystematische Steuervergünstigungen 1 iefert Surrey (1973), $\mathrm{S}$. $92 \mathrm{ff}$. Surrey macht bei seinen uberlequngen allerdings einen klaren Unterschied zwischen Steuervergünstiaungen im Sinne niedrigerer Steuersätze und Subventionen im Sinne direkter Obertragungen. Speziel1 Steuervergünstigungen führen zu folgenden Nachteilen: Mitnahmegewinne, Verteilungsungerechtigkeiten, we $i l$ hauptsächlich höher Verdienende davon profitieren; Marktverzerrungen und hohe Steuersätze, weil die Steuerbemessungsgrundlage eingeengt wird.

3) In der Konsequenz sollte bei größerer Unsicherheit über die Wirkungen von Steuerprivilegien auf ihren allokativen Einsatz verzichtet werden. Vgl. Brennan/McGuire (1975) und Folkers (1985) 
der Oberlegung, daß der Erwartungswert der Nutzenverluste aus einer fehlgerichteten Abweichung von der einheitlichen Besteuerung größer ist als der Erwartungswert der Nutzengewinne ${ }^{1}$ ). Dies resultiert aus der Tatsache, daB die Wohlfahrtsverluste einer fehlgerichteten Besteuerung im Quadrat des Steuersatzes ansteigen ${ }^{2)}$. In der Realität muß aufgrund von Monopolen, externen Effekten, Steuern und anderen statlichen Regulierungen mit erheblichen Informationsdefiziten gerechnet werden. Ein begründetes Wahrscheinlichkeitsurteil über die Richtung und das Außmaß erwünschter Korrekturmaßnahmen für angebliche und tatsächlich existierende Abweichungen vom Konkurrenzoptimum ist daher kaum möglich. Erstens stehen der Informationsgewinnung technische Probleme entgegen, die bereits in Kap. III diskutiert wurden, und zweitens ist die Informationsbeschaffung aller relevanten Daten mit prohibitiven Kosten verbunden ${ }^{3)}$. Eine steuerliche Sonderbehandlung, also auch die Subventionierung einzelner Teile einer Steuerbemessungsgrundlage, wird daher bei Ungewißheit und Risikoneutralität ${ }^{4)}$ nur zufällig zu Allokationsverbesserungen führen können. Daraus folgt aber auch, daß mit zunehmendem Informations-

1) Vgl. Brennan/McGuire (1975). Viele Bereiche der gegenwärtigen Transfersysteme scheinen exakt durch diesen Tatbestand gekennzeichnet zu sein. Vgl. dazu das Gutachten der Transfer-Enquête-Kommission (1981) und die kritische Würdigung von Lampert (1982), der konstatiert: "[...], daB zweitens das empirische Fundament für die Aufgabenerfüllung - u.a. die Ermitt]ung der Verteilungswirkungen [der Transfers (vom Verfasser eingefügt)] unzulänglich - genau genommen nicht vorhanden war..." S. 502

2) $\mathrm{Vgl}$. Anhang $B$

3) Vgl. Ng (1977), S. 1, Sohmen (1976), S. $436 \mathrm{f}$.

4) Sind dagegen mit bestimmten Aktivitäten unterschiedliche Erwartungswerte verbunden und müssen Risikofreude bzw. Risikoscheu mitberücksichtigt werden, so müssen die Optimierungsergebnisse von Brennan/ McGuire (1975) modifiziert werden. Vgl. dazu die Ergebnisse in Kap.VI. Die Risikoneigung der Entscheidungsträger spielt hier eine nicht unerhebliche Rolle. 
stand gezieltere Steuereingriffe möglich werden ${ }^{1}$ ). Eine ausreichende Informationsbasis zur Vergabe rationaler Subventionen scheint jedoch eher die Ausnahme als die wuinschenswerte Regel zu sein ${ }^{2}$ - unter anderem auch deshalb, weil auf der politischen Ebene oft gar kein Interesse an einer exakten Wirkungsanalyse besteht ${ }^{3)}$. Unter diesen Umständen kann man erwarten, daß Steuerprivilegien regelmäßig zu Nettonutzenverlusten führen werden ${ }^{4}$ ).

Schließlich kann gegen das Second-Best-Argument selbst eingewandt werden, daß es keine Angaben darüber macht, welche der vorgegebenen Restriktionen tatsächlich "unabänderbar" sind oder welche aus Grüden der politischen Opportunität bestehen bleiben. Hier ist also eine politisch-institutionelle Diagnose unabänderbarer ökonomischer Störungen gefordert. Wie bereits erörtert, ist der politische Prozeß jedoch kein unbestechlicher Ratgeber, und es erscheint geradezu verblüffend naiv, zu glauben, "die offizielle Anerkennung eines Tatbestandes als politisches Tabu könnte ohne Rückwirkungen bleiben" 5 )". Gerade die tabuisierten Steuervergünstigungen sind die Ursache

1) $\mathrm{Vgl} . \mathrm{Ng}(1977), \mathrm{S} .4 \mathrm{ff}$.

2) Vgl. Z.B. Lampert (1982), S. 475

3) "The less that is known the greater the incentive to ignore the effects and to take the tax decisions on the basis of other criteria - forgone revenues, effects on the budgets stance or perceptions about public reaction". Good (1980), Kap. 7, S. XVii. "Second little is known because there is little incentive to for actors, either insiders or outsiders, to know". Good (1980), Kap. 7, S. XVi

4) Vgl. zu einer umfassenderen Analyse Ng (1977), insbesondere S. 11

5) Sohmen (1976), S. 437. Mit "Rückwirkungen" sind hier vor allem neue Forderungen nach Steuervergünstigungen gemeint. Issing bemerkt hierzu: "Subventionen sind aufgrund ihres speziellen Charakters geradezu ein Einfallstor für mächtige Sonderinteressen, der Gesamtaufwand an derartigen Mitteln kann danach als ein Indikator dafür gelten, inwieweit der Staat bereits diesem Druck erlegen ist". Issing (1983), S. 56 
für weitere Subventionsforderungen bisher unberücksichtigter Gruppen.

Das Argument kann durch folgendes Beispiel illustriert werden: Es sei unterstellt, daB es bei der Einführung einer Subvention aus allokativen Aspekten heraus tatsächlich sinnvoll war, eine Subvention zu gewähren, daB aber im Laufe der Zeit die Allokationsstörungen verschwunden sind, so da $B$ ein Subventionsabbau sinnvoll wäre. Da die Abschaffung oder Reduktion der Subventionen zu Verteilungsverlusten bei den Betroffenen führt, werden sie sich gegen die beabsichtigten Maßnahmen wehren ${ }^{1)}$. Wenn die betroffene Gruppe einflußreich genug ist, wird es ihr gelingen, die Pläne der Regierung zu verhindern. Die bestehenden Subventionen werden nun aber selbst Quelle von Allokationsstörungen, da die ursprünglichen Allokationsverzerrungen nicht mehr existieren.Akzeptiert man die inzwischen ineffiziente Subvention als Datum, so müßten konsequenterweise "optimale" neue Steuern oder Subventionen zur Kompensation der Störwirkung alter Subventionen eingesetzt werden, um das bestmögliche Allokationsergebnis zu erzielen. Diese auch in der Realität beobachtbaren Folgereaktionen kann man als Subventionsspirale bezeichnen. Das Beispiel einer Subventionsspirale ${ }^{2)}$ demonstriert, daß eine optimale Steuer- und Subventionspolitik nicht nur

1) Beispielsweise bemerkt Gluch zu den Bausubventionen: "Diese staatlichen Subventionen sind nämlich, insbesondere be $i$ den hohen Wohnungspreisen in den Ballungsräumen, mittlerweile für viele Bauinteressenten notwendig, um die monatliche Belastung bei Erwerb der eigenen vier Wände auf ein tragbares Niveau zu senken". Gluch (1983), S. 74

2) Eine typische Kettenreaktion dieser Art kann man sich auch auf dem Wohnungsmarkt vorstellen, wo Mietpreisregulierung, Kündigungsschutz u.ä. den Marktmechanismus außer Kraft setzen und damit selbst Ursache für Subventionsforderungen werden. 
rein ökonomische Aspekte berücksichtigen darf, sondern auch die politisch-ökonomischen Reaktionen der Wirtschaftssubjekte beachten muB. Geschieht dies nicht, dann besteht die Gefahr, daB mit Allokationsargumenten immer neue Steuern und Subventionen eingeführt werden, die im Vergleich mit einer umfassenden politisch-ökonomischen Lösung eindeutig suboptimal wären. Aus diesem Grund, insbesondere aber wegen der allgegenwärtigen Informationsmängel, wurde auch versucht, die einfacher zu beurteilenden Allokationswirkungen der aggregierten Steuervergünstigungen $z u$ beschreiben und $z u$ messen ${ }^{1)}$. Dabei wurden die Wohlfahrtsverluste der aggregierten Steuervergünstigungen des aktuellen Steuersystems mit jenen einer "comprehensively based tax of equal yield and lower marginal rates" 2 ) verglichen. Die Wohlfahrtskosten der bestehenden Steuervergünstigungen werden dabei grob auf ca. $6,7 \%$ des steueraufkommens neschätzt ${ }^{3}$ ).

Die Wohlfahrtsverluste solch unsystematischer Steuervergünstigungen resultieren jedoch nicht nur aus der Verzerrung der relativen Preise durch die Steuervergiunstigung selbst ${ }^{4}$, sondern auch durch Steuererhöhungen in anderen Bereichen ${ }^{5}$, die den Steuerausfall kompensieren müssen und somit weitere Verzerrungen der relativen Preise nach sich zieien 6). Bedenkt man, daß die Allokationsverluste mit dem Quadrat der Subven-

1) Vgl. Browning (1979)

2) Browning (1979), S. 202. Implizit findet sich die Vorgehensweise auch bei Buchanan/Brennan (1980b), S. $43 \mathrm{ff}$.

3) Browning (1979), S. 200

4) Diese Problematik wird in den folgenden Abschnitten noch näher erläutert.

5) Vgl. Folkers (1985)

6) Beispielsweise sind hohe Grenzsteuerstätze bei der Einkommensteuer auch für die wachsende Steuerhinterzieinung und Schattenwirtschaft verantwortlich; gleichzeitig muß aber ihre Umverteilungswirkung angezweifelt werden, weil die effektive Progressivität weit unter der formalen liegt. $\mathrm{Vgl}$. Ben-Porath/Bruno (1977), S. 290 
tions-bzw. Steuersätze steigen ${ }^{1}$ ), so werden die Forderungen nach Subventionsabbau und Steuersenkung verständlich. Die einander kumulierenden Steuervorteile, nicht die einzelne Subvention, sind die Ursache starker Marktverzerrungen und Ineffizienz. "If capital gains and all other income sources were taxed at the same rates and unnecessary personal deductions were eliminated, it would be possible to bring the tax rates down to a maximum of 50 percent and still increase the progressivity of the individual income tax moderately. Tax reform along these lines improve horizontal as well as vertical equity, increase incentives to earn additional income through greater work effort and risk-taking, promote a better allocation of economic resources, and simplify tax administration and compliance"2).

Die vorgetragenen Argumente, insbesondere der politisch-ökonomische ProzeB der Subventionsvergabe ${ }^{3)}$, liefern eine ausreichende Basis für die Arbeitshypothese, daß zusätzliche Steuervergünstigungen, die in der Regel aus Verteilungsgründen gefordert und aus politischer opportunität gewährt werden, normalerweise die Ursache von Allokationsstörungen sind ${ }^{4}$ ).

1) Vgl. Anhang $B$

2) Break/Pechman (1975), S. 135 und Tab. 6-4, S. 131

3) Vgl. dazu auch Folkers (1985)

4) Siehe Buchanan/Brennan (1980), S. $47 \mathrm{ff}$. 
VII.2.5.3. Kapitalisierunaseffekte und Steuerreformen

Der Vorgang der Kapitalisierung von Steuerorivilegien ist ein Spezialfall des wesentlich allgemeineren Phänomens, daß eine Tendenz zur Kapitalisierung (bzw. Amortisation) ${ }^{1}$ ) von diskriminierenden staatlichen Eingriffen besteht. Diskriminierung in diesem Sinne bedeutet, daß nur bestimmten Personen (oder wirtschaftlichen Aktivitäten) Vergünstigungen gewährt bzw. Nachteile zugefügt werden, so daß ex ante gleichgestellte Wirtschaftssubjekte durch den Eingriff in ökonomisch ungleiche Positionen gelangen. Die daraus resultierenden ökonomischen und politischen Systemeffekte wirken dieser Ungleichbehandlung jedoch entgegen und können sie kompensieren oder gar überkompensieren.

\section{VII.2.5.3.1. Der Marktkapitalisierungseffekt}

Als Beispiel für die Kapitalisierung einer Subvention ${ }^{2}$ auf der Marktnachfrageseite kann die Förderung des Erwerbs von selbstgenutztem Wohneigentum herangezogen werden ${ }^{3)}$. Bei steigender Nachfrage und relativ konstantem Wohnungsangebot, d.h. bei einer unelastischen Angebotskurve ${ }^{4}$ steigen die Preise für Immobilien ${ }^{5)}$. Im Idealfall erhöhen sich die Preise so

1) Musgrave (1974), S. 348. Musgrave bezeichnet den Vorgang der Steueramortisation als "Neubewertung der relativen Kapitalwerte, die dann erfolgt, wenn ertragbringendes Vermögen einer diskriminierenden Steuer auf diese Einkünfte unterliegt".

2) Die tendenziell gleichen Effekte erhält man auch für Stücksubventionen.

3) Vgl. Leitlinien zur Neuregelung der steuerlichen Förderung des selbstgenutzten Wohneigentums, Bundesministerium der Finanzen 25 (1984), S. 4

4) Ein unelastisches Angebot besteht zumindest kurzfristig.

5) Die hier unterstellte Nachfrageerhöhung wird häufig durch Abwarten oder zeitliches Vorziehen der Nachfrage wegen günstiger Finanzierungsmöglichkeiten, nicht aber durch eine insgesamt höhere Nachfrage erzeugt. Vgl. Gluch (1983), S. 74 oder Härtel (1984), S. $235 \mathrm{ff}$. 
lange, bis die gewährten Steuervorteile kompensiert bzw. kapitalisiert sind ${ }^{1)}$. Die Gewinner dieser Subventionspolitik sind die früheren Wohnungseigentümer. Dasselbe Problem ergibt sich bei der subventionierten Wohnbauförderung, die einer kurzfristig konstanten Baukapazität der Bauunternehmen gegenübersteht. Die anlaufende Baukonjunktur führt auch hier nur zu Preiserhöhungen, ohne daß Struktureffekte erzielt werden, so daß es sich c.p. um Umverteilungseffekte hande $1 t^{2}$ ). Begünstigt werden in diesem Falle nicht die Bauwilligen, sondern die Bauunternehmer und die Alteigentümer der Grundstiicke, die entsprechend hohe Mitnahmegewinne realisieren können. Der Erfolg der Allokations-bzw. Verteilungspolitik hängt daher entscheidend von den Steigungen der Angebots-und Nachfragekurven auf den jeweiligen

1) Am Beispiel einer Bausubvention weist Gluch implizit auf dieses Phänomen hin: "In Immobilienteilen der überregionalen Zeitungen stellten die Bauträger in den zurückliegenden Wochen des vergangenen Jahres insbesondere die Vorteile heraus, die sich beim schnellen Kauf von Objekten bis etwa 300.000 Mark ergeben. Hier entfiel beim Erwerb im Jahr 1982 die gesamte Grunderwerbsteuer oder lag beträchtlich niedriger als nach der neuen Regelung. Etliche Anbieter erhöhen gerade im Dezember die Preise für diese Objekte nahezu exakt um den errechneten Vorteil". Gluch (1983), S. 74

2) Vgl. im Gegensatz dazu Goetz (1980), S. 61 ff., der mit der vollkommenen Kapitalisierung eines diskriminierenden Steuereingriffs grundsätzlich Struktureffekte und damit Allokationsverluste auf dem betreffenden Markt unterstellt.

3) Vgl. zu einem sehr illustrativen Beispiel antizipierter Steuervergünstigungen Bittker (1975), S. $416 \mathrm{ff}$. 
Märkten $a b^{1}$ ).

VII.2.5.3.2. Der institutionelle Kapitalisierungseffekt

Der institutionelle Kapitalisierungsprozeß ist ebenfalls eine Folge der diskriminierenden Steuerprivilegpolitik des States. Im Unterschied zum Marktkapitalisierungseffekt werden die Steuervorteile dabei jedoch nicht durch Marktprozesse aufgezehrt, sondern durch den Ressourceneinsatz politisch aktiver Wirtschaftssubjekte und Interessengruppen. Im politischen Kampf um Steuervorteile müssen von allen Interessengruppen Ressourcen eingesetzt werden ${ }^{2}$ ), um Verteilungsvorteile für die eigene Gruppe zu erringen. Dieses Verhalten ist in der Literatur als rent-seeking ${ }^{3)}$ bekannt. Je höher c.p.

1) Die bekanntesten Untersuchungen über Kapitalisierungseffekte finden sich im Zusammenhang mit der Tiebout-Hypothese. Val. Tiebout (1956), S. 416- 424. Oates (1969) argumentiert, daß sich die qualität und Quantität der öffentlichen Güter auf lokaler Ebene auf das Niveau der Vermögenswerte niederschlagen. Unter den Voraussetzungen des TieboutModells werden die Individuen in die Gemeinden ziehen, die die beste öffentliche Güterversorgung be $i$ sonst identischen Voraussetzungen aufweisen. Die Kapitalisierung der lokalen Renten hängt also von der Mobilität der Individuen (Nachfrage) und der Angebotsseite ab. Bei steigender Nachfrage und relativ konstantem Immobilienangebot erhöhen sich die Grundstücks- und Häuserpreise im Idealfall so lange, bis die gemeindespezifischen Ausgaben- und Steuervorteile kapitalisiert sind. Oates und die meisten empirisch arbeitenden Autoren haben die Kapitalisierungshypothese in der Tendenz bestätigt. Vgl. Pollakowski (1973), Hyman/ Pasour (1973), Oates (1973), Edel/Sclar (1974), King (1977) u.a. Manche Autoren ermittelten sogar eine Steuerkapitalisierung über $100 \%$. Vgl. dazu Church (1974).

2) Vgl. Z.B. Folkers (1983b), S. $196 \mathrm{ff}$. und Folkers (1985)

3) Buchanan definiert rent-seeking als "behavior in institutional settings where individuals efforts to maximize value generate social waste rather than social surplus". Buchanan (1980b), S. 4. Rent-seeking existiert in allen Gesellschaften und in vielen Spielarten. $\mathrm{Vgl}$. zu einigen Beispielen Krueger (1974), Posner (1975), Tullock (1975). 
die Reformkosten sind, um so geringer werden die verbleibenden Verteilungsvorteile aufgrund dieses Kapitalisierungseffektes.

"Reformkosten und eine höhere Mehrbelastung werden bis zu dem Punkt eingegangen, an dem ihr Zuwachs dem erwarteten Verteilungsvorteil gleich wird"1).

Beim insti.tutionellen Kapital isierungseffekt werden Transferzahlungen (z.B. Parteispenden) und Ressourcen nur zum Zweck der Umverteilung, nicht aber zu erhöhter Produktion verwendet. Ressourcen, die im Wettbewerb um Verteilungsvorteile verschwendet werden, sind aber volkswirtschaftliche Kosten. Die gesamtwirtschaftlichen Kosten individueller Steuerprivilegien würden also nicht wie in der traditionellen Sichtweise lediglich aus der Mehrbelastung bestehen; sie sind wesentlich höher einzuschätzen als häufig vermutet wird ${ }^{2}$ ).

Gleichzeitig resultiert aus der Kapitalisierung von Steuerprivilegien eine Ursache für ihr Beharrungsvermögen ${ }^{3)}$. Denn die Abschaffung von Steuervergünstigungen führt ganz offensichtlich zu Einkommens-bzw. Nutzeneinbußen für die jeweilige Gruppe. Die betroffenen Mitglieder der einzelnen Interessengruppen werden Einkommensminderungen den Funktionären als Versagen anlasten und möglicherweise finanzielle Kürzungen oder eine straffere organisation fordern. Verbandsfunktionäre haben

1) Folkers (1983b), S. 195

2) "Expenditures on the production of pressure are not Pareto optimal because all groups could be made better off by reduced expenditures". Becker (1983), S. 387. Um die Kosten des politischen Wettbewerbs zu vermeiden, sind Verhandlungen zwischen den Gruppen notwendig. Warum es häufig dennoch nicht zur Kooperation zwischen Interessengruppen kommt, erklärt Folkers (1985) in einem spieltheoretischen Ansatz.

3) Vgl. Folkers (1985) 
deshalb ein existentielles Interesse an der Aufrechterhaltung alter Steuervergiunstigungen. Sie werden deshalb gezielte Informationen und adäquate Drohstrategien gegenüber der Regierung und Bürokratie einsetzen, um ihre formalen Steuerprivilegien zu verteidigen. Dies gilt um so mehr, wenn die Verteilungsvorteile, die zur Disposition stehen, bereits kapitalisiert sind. In diesem Fall erleiden die betroffenen Steuerzahler echte Verteilungsverluste gegenüber der Ausgangssituation ohne staatliche Eingriffe ${ }^{1}$ ).

Dieses Faktum markiert auch einen Ursachenkomplex für den permanenten Reformdruck der Interessengruppen auf die politischen Akteure ${ }^{2)}$. Der Wunsch nach Steuerprivilegien resultiert einerseits aus dem nutzenmaximierenden Verhalten der Individuen selbst und wird andererseits durch die Kapitalisierungseffekte verstärkt. Die Kapitalisierung von Steuerprivilegien erodiert Einkommensvorteile und ist eine wesentliche Erklärung für den dynamischen Anreiz zum Kampf um immer neue Steuervorteile ${ }^{3 \text { ). }}$.

\section{VII.2.6. Zur Dynamik von Steuerreformen}

Die konkreten Reformaktivitäten und der Zeitpunkt des Eingreifens von Interessengruppen hängen von vielfältigen Faktoren ab ${ }^{4}$,

1) Vgl. Folkers (1985)

2) Vgl. zu einer detaillierten empirischen Analyse der Entwicklung von Steuersystemen und insbesondere von Steuervergünstigungen Alt (1983), S. $187 \mathrm{ff}$.

3) Vgl. Folkers (1985)

4) Bereits in Kap. VI wurde gezeigt, daß Entscheidungen bei Unsicherheit auch von persönlichen Faktoren (wie z.B. der Risikobereitschaft einzelner Interessenvertreter) abhängen können. Die Vielzahl von Einflußfaktoren spricht auch gegen ein formales, positives Reformmodel1, weil die Beschreibung des unsystematischen Willensbildungsprozesses nur durch die Abstraktion und damit durch Abstriche von seinen typischen Charakteristika erkauft werden kann. Vgl. zu dieser Einschätzung Folkers (1985). 
die für die Höhe der entstehenden Reformkosten maßgeblich sind: ${ }^{1}$

a) dem Informationsstand in der offentlichkeit und innerhalb der Verbände,

b) der Komplexität der bestehenden Steuergesetzgebung,

c) der Anzahl der Reformbeteiligten,

d) dem Organisationsgrad anderer Interessengruppen und der eigenen Organisation ${ }^{2)}$,

e) dem vermuteten Einfluß potentieller Reformgegner und

g) dem budgetären Spielraum der Entscheidungsträger.

Diese Auflistung erhebt keinen Anspruch auf Vollständigkeit, sie dürte jedoch entscheidende Faktoren enthalten, die das Verhalten der Interessengruppen, die Dynamik und das Ergebnis im Kampf um Steuervorteile determinieren. Sie kennzeichnen das nahezu unerschöpfliche Spektrum gruppenspezifischer Aktivitäten im politischen willensbildungsproze ${ }^{3}{ }^{3}$. Insbesondere die Informationsdefizite der Steuergesetzgebung, ihre Komplexität ${ }^{4}$ )

1) Vgl. zu einer ähnlichen Analyse Folkers (1983b), S. $197 \mathrm{ff}$.

2) Vgl. z.B. Tullock (1983), S. 2

3) Vgl. Z.B. Becker (1983) oder zu einer spieltheoretischen Analyse potentieller Verhaltensweisen von Interessengruppen im Steuerreformprozeß, Folkers (1985), oder Johanson (1979), S. $506 \mathrm{ff}$.

4) Der Kreis der Informierten wird immer exklusiver und die Entscheidungen werden somit bei immer geringeren Kontrollmöglichkeiten der Allgemeinheit gefällt. Gleichzeitig fehlt allerdings auch den Insidern immer mehr der Oberblick. Auf jeden Fall aber führt die Komplexität dazu, daß es auch theoretisch besser wäre, Entscheidungen in kleinen Zirkeln zu fällen. Vgl. Bernholz (1972). S. 226. 01son kommt in einer umfassenderen Analyse des Verhaltens von Interessengruppen zu dem Ergebnis: "The accumulation of distributional coalitions increases the complexity of regulation, the role of government, and the complexity of unterstandings, and changes the direction of social evolution.", 0lson (1982), S. 74. $\mathrm{Vgl}$. zu einer umfassenden Analyse dieses Problems Miner/Charlice (1978), Becker (1983), S. 385 und 395 oder Folkers (1983b), S. 198. Zu einem politisch aktuellen Urteil siehe z.B. Weilepp (1932). 
sowie die Vielzahl der Interessengruppen stellen eine güstige Bedingungskonstellation für permanente Forderungen nach steuervergünstigungen dar ${ }^{1}$ ). Denn aufgrund der hohen Informationskosten werden normalerweise nur Allokations- und Verteilungswirkungen von Steuervergünstigungen wahrgenommen, die die eigene Gruppe betreffen ${ }^{2}$. Die Opposition anderer Verbände bleibt daher gering. Stattdessen werden sich die benachteiligten Interessengruppen um eigene Steuervorteile bemuihen ${ }^{3)}$ und tragen so zur "Akzeleration" des Reformprozesses be ${ }^{4}$ ).

Die Dynamik kleiner Reformen wird vor allem durch eine "Reformillusion" seitens der Zensiten bzw. Interessengruppen in Gang gehalten ${ }^{5}$ ). Das Schlagwort "Reformillusion" beschreibt die trügerische Hoffnung der organisierten Steuerzahler, durch die ihnen gewährten Steuerveraunstigungen auf Dauer Nettoverteilungsvorteile erhalten zu können. Bei gleichem steueraufkommen kann dann die sukzessive Vergabe von Steuerprivilegien an alle

1) Vgl. Folkers (1985)

2) Vgl. dazu Folkers (1983b), S. 198

3) Dies kann offiziell durch speziell eingesetzte Kommissionen oder inoffiziell in persönlichen Gesprächen, legal in Form von Stellungnahmen der betroffenen Interessengruppen oder illegal durch Bestechung geschehen. Vgl. dazu Riedel (1982). Für die Reform des Körperschaftsteuergesetzes von 1977 wurde die Erhard-Kommission eingesetzt, die auch mit Vertretern verschiedener Interessengruppen besetzt war. Ebenda, S. $188 \mathrm{f}$. Ferner existieren Stellungnahmen der betroffenen Interessengruppen. Ebenda S. $84 \mathrm{ff}$. Die Bedeutung des persönlichen Gespräches wird deutlich bei Reese (1980), S. 21: "[...] if Surrey [vg]. Surrey (1973)] had taken 10 percent of the time he spent on rewriting correspondence and spent it drinking with congressmen, he would have seen much better off" [with his tax reform ideas (der Autor)].

4) Vgl. Good (1980), Kap. 2, Folkers (1978)

5) Vgl. Folkers (1983a) 
Interessengruppen und Wählerschichten nur durch höhere Steuern in anderen Bereichen kompensiert werden. Die heimlichen Steuererhöhungen (insbesondere im Bereich der Lohnsteuer) und die jüngsten Mehrwertsteuererhöhungen mögen als Beispiele dienen. Unter den genannten Voraussetzungen führt eine solche Subventionspolitik bei der Mehrheit der Steuerzahler im besten Falle lediglich zu einer Umstrukturierung ihrer Steuerzahllast, jedoch bei wesentlich höherer Mehrbelastung, so daß die Gesamtbelastung steigt ${ }^{1}$ ).

Zur Dynamik des Steuerreformprozesses trägt weiterhin bei, daB es für manche Interessengruppen selbst bei Kenntnis der skizzierten Zusammenhänge rational wäre, weitere Steuerprivilegien anzustreben, weil sie trotz des allgemeinen Subventionswettbewerbes Nettogewinner bleiben. Andere müssen nachziehen, um nicht völlig leer auszugehen, d.h. um die unvermeidlichen Reform- und Verteilungsverluste zu minimieren. Die Wahrscheinlichkeit, daß es zu Verhandlungslösungen kommt, die den po1 it ischen Subventionswettbewerb verhindern oder zumindest einschränken, erscheint daher sehr gering, zumal die Zahl der Vergünstigungen und Gruppen zu groß ist, um freiwillige, kooperative Lösungen anzustreben ${ }^{2}$ ).

1) Neben zunehmenden Allokationsverlusten entstehen aber auch erhebliche Verteilungsungerechtigkeiten, weil die Verteilungswirkungen der kumulierenden Steuervergünstigungen nicht einmal mehr tendenziell eingeschätzt werden können. Vgi. Z.B. Lampert (1982), S. 480 f.

2) $\mathrm{Vgl}$. die spieltheoretische Analyse von Folkers (1985). Dort wird die Problematik asymmetrischer Spiele eingehend analysiert. 
Was fehlt, sind Institutionen, die den Kampf um Subventionen aus der Sicht der Reformteilnehmer sinnlos erscheinen lassen und die Politiker vor ihrem eigenen ineffizienten Handeln schützen ${ }^{1)}$.

VII.3. Konzeptionelle Mängel des politisch-ökonomischen Steuerreformprozesses

Im steuerpolitischen Willensbildungsprozeß muß damit gerechnet werden, daß wesentliche Teile der Steuergesetzgebung nicht aufgrund von sozialen Steuernormen, sondern durch individuelle, eigennützige Interessen der Reformbeteiligten entstehen. Der Wunsch nach spezifischen (Verteilungs-) Ergebnissen stellt allerdings die Konzeption eines rationalen Steuersystems auf den Kopf. Zielsysteme sollten vorgegeben und die Ergebnisse der (optimalen) Besteuerung unter genau spezifizierten (realitätsnahen) Annahmen abgeleitet werden ${ }^{2}$ ). Die Umkehrung dieses Rationalitätsprinzips wird einen iterativen ProzeB zur Folge haben, in dessen Verlauf die Politiker jene Zielvorgaben wählen, die eine politisch opportune Instrumentenwahl und Ver-

1) Bezeichnend hierfür ist die Subventionspolitik der Bundesregierung $1983 / 84$, die trotz vieler Versprechungen bisher keinen Subventionsabbau bewirken konnte.

2) Vgl. dazu Haller (1980) 
teilungsstruktur zulassen ${ }^{1)}$. Der normative Anspruch der OT wird auf diese Weise durch eine positive "wenn-dann" Beziehung ersetzt, selbst wenn man theoretisch ermitteln könnte, auf welchem Verteilungsziel die gewüschte Ergebnisstruktur basiert. (Vgl. Kapitel IV dieser Arbeit).

Auch der Vorschlag, Normen dann zu akzeptieren, wenn sie und ihre Implikationen bzw. Verteilungsergebnisse vernüntig und konsensfähig seien ${ }^{2}$, ändert an den prinzipiellen Mängeln aktueller Steuerreformprozesse nichts. Das methodische Prinzip beruht nämlich darauf, die Norm mittels ihrer Verteilungsergebnisse zu überprüfen. Diese Auffassung einer Normakzeptanz bringt im konkreten Fall folgende Schwierigkeit: Woher weiB man, daB die aus einer als gerecht vermuteten Norm abgeleitete Steuerlastverteilung ungerecht ist ${ }^{3)}$ ? Und wer stel1t dies fest?

1) Diese Vermutung kann durch ein Beispiel während des Willensbildungsprozesses der Körperschaftsteuerreform 1977 verdeutlicht werden: "In der offiziellen Begründung des Regierungsentwurfs vom Oktober 1973 findet sich zwar eine steuersystematische Argumentation für die Verweigerung des Vollzugs der Emissionskosten, der empirische Teil der Studie läßt jedoch den Schluß zu, daß die volle Abzugsfähigkeit aus fiskalischen Gründen verweigert und die steuersystematische Begriindung nachgeschoben wurde", Riedel (1982), S. 264. Kennzeichnend sind auch die Außerungen von Littmann, daß die Finanzpolitik während der Konsolidierungs - und Reformmaßnahmen dort aktiv war, "wo es politisch durchsetzbar war, und nicht immer da, wo es vernünftig gewesen wäre" . Littmann (1984), S. 70. Interessant ist auch, daß aufgrund von Steuerillusionen nicht die materielle sondern die formale Inzidenz Grundlage steuerpolitischer Debatten ist. Sicherlich erklären sich somit viele Impulse in der Reformdiskussion aus dem Bestreben nach "politisch ertragreichen formalen Inzidenzbeziehungen". Folkers (1983b), S. 201

2) Vgl. Pfähler (1978), S. 33 ff.

3) Diese Frage stellt sich auch Pfähler (1978), S. 35 
Der Vorstellung, man könne durch ein Trial-oder Error-Verfahren ein allgemein guiltiges Gerechtigkeitspostulat ermitteln, muß entgegengehalten werden, daß es in einer konkreten situation eben keinen Konsens über Gerechtigkeitsideale gibt ${ }^{1)}$. Dies liegt in der Natur konfligierender Interessen eigennitziger Individuen. Je nachdem, welche Position der Einzelne in einer Gesellschaft einnimmt, wird er allein schon aufgrund seines Eigeninteresses (bewuBt oder unbewuBt) andere Situationen als gerecht betrachten.

Außerdem stellt sich die Frage, warum man überhaupt Normen ableiten will, wenn sie nur dann akzeptiert werden, wenn ihre Steuerlastverteilung allgemein als gerecht akzeptiert wird. In diesem Fall würden Normen durch die konkrete Ergebnisstruktur restringiert. Ihre Erfiullung ist an sich nicht mehr erstrebenswert. Der Wunsch nach einer bestimmten Ergebnisstruktur ist dann aber auch nicht mehr logisch ableitbar, sondern nur noch durch Willkür gekennzeichnet. Willkür jedoch ist in höchstem Maße inevident und konsensunfähig. Gerechtigkeit muß, um Konsens zu erreichen, daher auf der Normebene definiert sein, nicht auf der Ergebnisebene.

Wird Konsens im Sinne der Einstimmigkeit interpretiert, so erscheint eine Lösung im laufenden politischen ProzeB nicht möglich. Nur auf der konstitutionellen Ebene besteht ein "ehrliches Interesse" an einer Einigung aller Beteiligten. Dies gilt sogar trotz (bzw. wegen) des Eigeninteresses der

1) Gerade wegen dieses Problems hat Rawls die Idee des "veil of ignorance" entwickelt, die auch als Grundlage für die institutionelle Steuertheorie dient. Vgl. Rawls (1971), Kap. 3 und Buchanan (1978) 
Individuen hinter dem Schleier der Unwissenheit ${ }^{1}$ ). Die Notwendiakeit fiskalischer Regelungen konstitutioneller Art wird damit erneut beleqt.

\section{VII.4. Zusammenfassung}

Im vorliegenden Kapitel wurde der Versuch unternommen, ein realitätsbezogenes Bild des steuerpolitischen Willensbildungsprozesses zu entwerfen. In diesem Zusammenhang konnte festgestellt werden, daß der finanzpolitische Entscheidungsspielraum der Politiker trotz der bestehenden politischen und fiskalischen Restriktionen nur unzureichend begrenzt wird, so daB eine unerwünschte Ausweitung statlicher Aktivitäten und die zunehmende Unsystematik des Steuerreformprozesses kaum verhindert werden können. Die Oberlegung wird auch dadurch bekräftigt, daß man in der Realität keinesfalls von einer eindimensionalen, eindeutig spezifizierten Zielfunktion des Fiskus ausgehen kann, wie es in den OT-Modellen bzw. Leviathan-Modellen unterstellt wird. Statt dessen muß man damit rechnen, daß sich Politiker und Bürokraten die Entscheidungsbefugnis miteinander teilen müssen, und daß die verschiedenen Zielvorstellungen und Interessen beider Gruppen häufig miteinander konfligieren. Wenngleich der Wunsch nach zunehmenden Steuereinnahmen durchaus mit einem Mehrheitswahlsystem und der Monopolmacht der Bürokratie kompatibel ist, kann diese systemimmanente Tendenz keinesfalls eindeutig der systematischen Durchsetzung statlicher Leviathanzielsetzungen zugeschrieben werden ${ }^{2}$ ).

1) "At the netrinate constitutional level of decision, the implied requirement of consensus prevents the partisan struggle among group interests that characterizes operational decision" Buchanan/Tullock (1962), S. 285/286. Vgl. in diesem Zusammenhang auch Frey (1980), S. 52

2) Denn relativ zunehmende Steuereinnahmen oder das Wachstum der Staatsausgaben könnten auch Ausdruck einer preisunelastischen Nachfrage nach arbeits intensiven staatlichen Dienstleistungen der Steuerzahler sein. Vg1. Borcherding (1981), Blankart (1977), Baumol (1967), Felderer (1979) und ergänzend Windisch (1980 I und II), sowie Bernholz (1981) 
Wegen der zumindest partiellen Wählerabhängigkeit der Politiker entsteht jedoch das Dilemma, daß steuerpolitische Entscheidungen mehrheitenopportun und daher in aller Regel auch norminkonsistent gefällt werden. Die Befriedigung gruppenspezifischer Steuerinteressen, gepaart mit politischem Mehrheitsdenken und bürokratischen Budgetwünschen sind eine wesentliche Ursache für eine unsystematische und ungerechte Steuerstruktur, die als Vehikel zur Verfolgung von eigensüchtigen Zielen dient. Darüber hinaus beruhen viele Zufälligkeiten im Steuerreformprozeß auf Informationsdefiziten seitens aller Reformbeteiligten. Es müssen daher institutionelle Regeln geschaffen und in den Verfassungsrang erhoben werden, die den steuerpolitischen Handlungsspielraum von Politikern und Bürokraten einengen und sie zu normkonsistentem Handeln zwingen. Eine konsensfähige Steuerverfassung ${ }^{1}$ sollte daher das Ziel einer umfassenden Steuerreform sein.

Bie Tendenz zu inkonsistenten Steuerreformentscheidungen wird aber auch durch Konsumenten und Produzenten im SteuerreformprozeB verstärkt. Ausgehend von der Nutzenmaximierungshypothese wurde unterstellt, daß das zentrale Interesse von Steuerzahlern darin besteht, ihre Gesamtsteuerlast zu minimieren. Dieses individuelle Steuerreformziel wird an Interessengruppen delegiert, die die Wünche ihrer klientel im Steuerreformprozeß durchzusetzen suchen. Zu diesem Zweck könnten sie versuchen, allgemeine Steuertarifänderungen, Begrenzungen der Steuerbemessungsgrundlagen u.ä. anzustreben. Da die Reformkosten für eine allgemeine Steuerlastsenkung für einzelne Gruppen relativ hoch, und das Nettoergebnis einer Reform daher ungewiB sein werden, rückt die individuelle Steuerprivilegvergabe des States zur Erzielung von

1) Eine Steuerverfassung wäre ideal, weil der isolierte Einsatz einzelner Verfassungsregeln häufig nicht die gewünschten Erfolge zeitigt. Vgl. dazu Kap. IX. 
gruppenspezifischen Verteilungsvorteilen in den Mittelpunkt der Reformdiskussion.

Dies bedeutet jedoch nicht, daß keine wirtschaftspolitischen Situationen vorstellbar wären, die es den Finanzpolitikern ratsam erscheinen lassen könnten, allgemeine Steuersenkungen anzustreben. Derartige Vorhaben sind beispielsweise in Zeiten tiefer Wirtschaftskrisen denkbar, wenn sich die Regierung durch allgemeine Steuersenkungen die Unterstützung der relevanten Gesellschaftsgruppen verschaffen will. In dieser Lage kann es für die Regierung von Vorteil sein, auch die Opposition in die Verantwortung für die weitere Wirtschaftsentwicklung miteinzubeziehen, so daß eine gemeinsame Steuerpolitik und somit allgemeine Steuerentlastungen wahrscheinlicher werden. Ein Blick auf die steuerpolitische Realität zeigt jedoch, daß Reformen, die zu einer allgemeinen Steuerlastminderung führen - auch unter Berücksichtigung der gegenwärtigen Reformbemühungen in der Bundesrepublik Deutschland und in den USA - nur in Ausnahmefällen zu erwarten sind.

Die Regierung wird in der überwiegenden Zahl der Fälle im Rahmen ihres finanziellen Handlungsspielraumes solche (kleinen) Steuerreformen anstreben, die eine sukzessive Steuerentlastung jener Gruppen bewirken, von deren Unterstützung sie sich den Wahlsieg verspricht. Der entscheidende Nachteil dieses Reformkonzepts besteht darin, daß steuervorteile, die aus egoistischen Motiven heraus angestrebt werden, nur zufällig in den Rahmen einer normativ begründeten Steuerpolitik passen können.

Darüber hinaus verursachen Steuervergünstigungen gemessen an einer umfassenden Steuerbemessungsgrundlage mit niedrigeren Steuersätzen bei gleichem Steueraufkommen Allokationsstörungen. Aber auch die Verteilungswirkungen der Steuervergünstigungen sind nicht so eindeutig, wie es auf den ersten Blick erscheinen mag. Beispielsweise kann sich aufgrund der Steuervergünstigungen die Nachfrage nach dem subventionierten Gut erhöhen, so daB auch der Marktpreis steigt, und die Steuer- 
vorteile somit kapitalisiert werden könnten. Darüber hinaus verschlingt der Kampf um Verteilungsvorteile auf der politischen Ebene Ressourcen. Im AusmaB des Ressourceneinsatzes im Wettbewerb um Steuervorteile ergibt sich ein institutioneller Kapitalisierungseffekt, der die Verteilungsvorteile der spezifischen Gruppen weiter schmälert. Das bedeutet, daB die Gesamtkosten der Subventionspolitik weit höher als bisher eingeschätzt werden muissen.

Bei gegebenem Steueraufkommen muß damit gerechnet werden, daß die Verteilungsgewinne der vielfältigen Subventionen für alle Bevölkerungsgruppen durch Nutzenverluste in Form allgemeiner Steuererhöhungen in anderen Bereichen kompensiert werden. Diese Tendenz wird durch die Kapitalisierung der Steuerprivilegien verschärft. Die Erosion der Einkommensvorteile liefert außerdem Argumente für das Beharrungsvermögen alter Steuerprivilegien und für die Dynamik im Kampf um neue Vorteile. Diesem ineffizienten und ungerechten Verteilungskampf kann nur durch konstitutionelle Regeln Einhalt geboten werden ${ }^{1)}$. Nur sie reduzieren die Attraktivität des Ressourceneinsatzes in Wettbewerb um Steuerprivilegien und zwingen gleichzeitig die finanzpolitischen Entscheidungsträger normkonsistentem Handeln.

1) Vgl. Folkers (1985) 


\section{STEUERVERFASSUNG ALS STEUERREFORMPROGRAMM}

Mit der vorliegenden positiven Analyse des steuerpolitischen Willensbildungsprozesses wurde die Notwendiakeit fiskalischer Regeln belegt. Offensichtlich werden auch in Demokratien die Präferenzen der Bürger nur insoweit respektiert, als dies für den Wahlsieg und somit für eigene Ziele der politischen Aktoren förderlich ist ${ }^{1)}$; dies gilt trotz demokratischer Wahlen und anderer verfassungsrechtlicher Restriktionen, die die fortschreitende Unsystematik des Steuersystems jedoch nicht verhindern können. Je besser es gelingt, die systematischen Mängel des demokratischen Steuerreformprozesses zu analysieren, um so gezielter können die Konstruktionsfehler der fiskalischen Restriktionen behoben und neue Verfassungsregeln abgeleitet werden ${ }^{2}$ ). Die Bedeutung positiver Reformmodelle wird besonders deutlich, wenn man sich vor Augen hält, daß im Falle eines wohlwollenden Planers mit vollkommenen Informationen keinerlei fiskalische Verfassungsregeln notwendig und wünschenswert sind, während sie im Falle eines Leviathan die letzte Rettung vor totaler Ausbeutung darstellen.

Nun hat die vorliegende Analyse auch die These vom Leviathan nicht bestätigen können; das Plädoyer für konstitutionelle Steuerregeln entspringt daher nicht primär dieser Maximierungshypothese, wie es bei Brennan/Buchanan geschieht ${ }^{3}$ ), sondern resultiert aus der Erkenntnis eines unsystematischen und inkonsistenten steuerpolitischen Willensbildungsprozesses, der dringend geeignete "Spielregeln" für die Verfolgung von Effizienz- und Verteilungszielen benötigt. Gleichwohl entspricht die
1) Vgl. Folkers (1984), S. 501
2) Vgl. Folkers (1984), S. 502
3) Vgl. Brennan/Buchanan (1980), insbesondere Kap. 2 
Logik von fiskalischen Verfassungsregeln in der Demokratie in weiten Bereichen den Begründunaszusammenhängen im Leviathan-Modell. Wie aus den existierenden Finanzverfassungen ersichtlich wird, gilt auch im demokratischen Staatswesen, daß die an die Regierung übertragene Macht der Besteuerung durch geeignete Steuerinstitutionen zum Wohle der Bürger beschränkt werden muB. Allerdings sind die in Kapitel $V$ behandelten ergebnisbezogenen fiskalischen Begrenzungen in einer Demokratie von etwas geringerem Interesse, weil erstens ihre starren und z.T. willkürlichen Grenzen relativ geringe Anpassungsmöglichkeiten des Staates an sich ändernde ökonomische Daten bewirken, und weil sie zweitens keine Garartie fur ein effizientes steuersystem sein müssen"1). Bei einem unbegründeten Einsatz dieser Regeln besteht eher die "Gefahr, daß damit auch neue Fehlentwicklungen initiiert werden" 2 ). Aufgrund dieser potentiellen Opportunitätskosten müssen fiskalische Verfassungsregeln bezüglich ihrer politisch ökonomischen Wirkungen in den Folgeperioden untersucht werden. Je restriktiver sie formuliert werden, um so größere Wohlfahrtsverluste können aus der verminderten Flexibilität wohlwollenden staatlichen Handelns erwachsen ${ }^{3)}$. Daher müssen die erwarteten Wohlfahrtsverluste mit den erwarteten Wohlfahrtsgewinnen aus der Beschränkung des demokratischen Handlungsspielraums verglichen werden. Bei einem erwarteten Nettogewinn ist die jeweils vorgeschlagene Begrenzungsregel (vgl. z.B. Abschnitt VIII.2.) anzuweriden.

1) $\mathrm{Vgl}$. Kapitel $\mathrm{V}$

2) Folkers (1983c), S. 169. Als Beipiel für diese Behauptung kann die Analyse der Wirkungen von Proposition 13 dienen, siehe Folkers (1983a), Kap. IV.

3) Buchanan sieht dieses Problem, hält es aber offensichtlich für unbedeutend. Vgl. Buchanan (1983), S. 10 f. und Buchanan/Brennan (1981), S. $4 \mathrm{f}$. 
Zur Oberprüfung des Spektrums potentieller Effizienz- und Verteilungsstrukturen variierender ökonomischer Rahmenbedingungen kann die OT einen nützlichen Beitrag leisten ${ }^{1)}$. Ist auf konstitutioneller Ebene ein zu breites Spektrum potentieller Effizienzverluste oder Verteilungsergebnisse in der Zukunft zu befürchten, so werden risikoscheue Individuen nicht mehr bereit sein, eine derartige konstitutionelle Steuerregel zu akzeptieren. Im Gegensatz zu Brennan/Buchanan kann nicht erwartet werden, daß Verfassungsregeln völlig losgelöst von ihren Ergebnis strukturen bestimmt werden ${ }^{2}$ ). Die einstimmige Verabschiedung von Steuerregeln ist dann zu erwarten, wenn die Auswirkungen dieser Regeln im laufenden politischen Prozeß einigermaßen überschaubar sind ${ }^{3)}$. Denn Verfassungsregeln sind nicht als solche wünschenswert, sondern nur deshalb, weil sie eine möglichst positive Ergebnisstruktur in späteren Perioden erwarten lassen bzw. Weil sie die schlimmsten Auswüchse eines unkontrollierten Leviathan verhindern sollen.

VIII.1. Die Funktion einer Steuerverfassung oder die Verfassungsregeln als institutionelle Second-Best-Regeln

Auf der konstitutionellen Ebene bietet sich die Chance, ökonomische Störungen, Marktverzerrungen und vermeintliche Restriktionen, die durch politische Institutionen erzeugt werden, aus dem Tabukatalog des laufenden finanzpolitischen Entscheidungsprozesses herauszunehmen und (die politischen Institutionen selbst) auf ihre finanzpolitischen Wirkungen hin zu untersuchen. Dieser Versuch wurde mit der vorliegenden positiven Steuerreformanalyse unternommen. Dabei wurde gezeigt,

1) Vgl. Z.B. die Rechenexempel von Ricketts (1981) oder die Sensitivitätsanalyse von Harris/McKinnon (1979)

2) Tatsächlich wird diese Sichtweise explizit bei Buchanan (1983), S. 10 bestätigt.

3) Dies bedeutet nicht, daß man die Position einzelner Individuen in den Folgeperioden kennen müßte, sondern nur, caij die Streuung der Ergebnisse absehbar sein müßten. $\mathrm{Vgl}$. dazu z.B. Kap. IV, wo die Ergebnisstrukturen einzelner Wohlfahrtsfunktionen diskutiert werden. 
daß viele Aktivitäten und Entscheidungen der demokratischen Steuerinstitutionen keinesfalls im Sinne eines wohlwollenden Planers durchgeführt werden. Diesem "Staatsversagen"1) sollen, in Analogie zum "Marktversagen"2), konstitutionelle Steuerregeln gegenübergestellt werden, die als Korrekturmechanismen der unsystematischen politischen Entscheidungsprozesse zu verstehen sind und die schlimmste Ineffizienz und Ungerechtigkeit zielinadäquater Steuerpoi itik verhindern sollen. Je größer die tatsächlichen oder die in Zukunft erwarteten Abweichungen von einer Feasible Best-Lösung eingeschätzt werden, d.h. je ineffizienter die Steuerpolitik wird, um so restriktiver werden die Verfassungsregeln ausfallen. Schlimmstenfalls müßten daher Vorkehrungen gegen einen Leviathan eingeführt werden.

Nun wären die strengsten konstitutionellen Steuerregeln als Schutz vor staatlicher Willkür für freiheitliche Gesellschaftsordnungen uneingeschränkt wünschenswert, wenn sie nicht gleichzeitig eine flexible optimierung steuerpolitischer Entscheidungen, wie sie z.B. von der OT angestrebt werden, erschweren könnten. Gemessen an der idealen (und daher nicht realen) Institution des wohlwollenden Diktators, sind fiskalische Verfassungsregeln nur zweitbeste (aber notwendige) Institutionen. In Anbetracht der positiven Analyse des steuerpolitischen Willensbildungsprozesses erscheint jedoch der hypothetische Vergleich zwischen einer wohlwollend regierten Welt ohne fiskalische Restriktionen, in der permanent ein Wohlfahrtsmaximum realisierbar wäre, und der ceteris paribus gleichen Welt mit Verfassungsschranken irrelevant. Unterstellt man statt dessen einen unsystematisch bzw. sukzessiv ausbeutenden Leviathan als Referenzmodell, so verkehrt sich das Vergleichsergebnis ins Gegenteil. Auf konstitutioneller Ebene besteht daher die Aufgabe, auf der Basis einer positiven Steuerlehre jene Steuerregein abzuleiten, die den maximalen Erwartungswert der gesellschaftlichen Wohlfahrt garantieren.

1) Vgl. Musgrave (1981a), S. 78 f. und Folkers (1983c), S. 167

2) Vg1. Musgrave $(1981 a)$, S. 78 
Die Kritik an der Inflexibilität konstitutioneller Steuerregeln muB somit relativiert werden. Eines ihrer Ziele besteht gerade darin, den Entscheidungsspielraum einer unsystematischen Steuerpolitik zu begrenzen. Das geschieht auch dadurch, daß die Politiker den auBerparlamentarischen Machtpotentialen nur noch im Rahmen der Verfassungsregeln nachgeben dürfen. Gemäß der Theorie des Zweitbesten wird von den konstitutionellen Steuerregeln erwartet, daß sie die "Verzerrungen" des Mehrheitswahlsystems kompensieren und so das tatsächliche Ergebnis des Gesamtsystems verbessern ${ }^{1}$ ).

\section{VIII.1.1. Einstimmigkeit und Effizienz von Verfassungsregeln}

Das entscheidende Kriterium für eine Steuerverfassung ist ihre einstimmige Verabschiedung. Einstimmig beschlossene Verfassungsregeln sind pareto-optima ${ }^{2}$ ). Die Zustimmung der Individuen hinter einem Schleier der Unwissenheit zur Einführung bestimmter Verfassungsregeln impliziert, daß sie sich von der Durchsetzung dieser Regeln ein höheres Wohlfahrtsniveau versprechen als ohne die Regeln ${ }^{3}$. Das Aushandeln der optimalen konstitutionellen Steuerregeln kann analog zu den Tauschvorgängen am Markt interpretiert werden. Tauschvorgänge werden immer einstimmig beschlossen und genügen bei rationalen Individuen, die ihren Nutzen maximieren, dem Pareto-kriterium. Die einstimmige Verabschiedung einer Steuerverfassung garantiert daher die Effizienz der darin enthaltenen Verfassungsregein.

1) Vgl. Folkers (1984), S. 505

2) Vgl. Buchanan (1983), S. 10

3) "Agreement on a change in the rules within which exchanges are allowed to take place would be a signal that pattern of outcomes reached or predicted under the previousiy existing set of rules are less preferred or valued than the pattern expected to be generated under the rule-aschanged". Buchanan (1983), S. 10 
VIII.1.2. Einstimmigkeit und Gerechtigke it von Verfassungsrege In

Einstimmig verabschiedete konstitutionelle steuerregeln sind nicht nur effizient, sie sind auch gerecht ${ }^{1)}$. Da jedes Individuum ein Vetorecht hat, kann keine Entscheidung gegen sein ethisches oder moralisches Empfinden getroffen werden. Darüber hinaus haben alle Individuen hinter dem Schleier der Unwissenheit ein Interesse, sinnvolle, möglichst gerechte Regeln zu verabschieden, da sie ja selbst nicht wissen, ob sie in den Folgeperioden möglicherweise zu den Benachteiligten zählen ${ }^{2}$.

Eine andere Frage ist allerdings, ob Individuen, die auf konstitutioneller Ebene einer Steuerregei zugestimmt haben, diese Regel auch in den Folgeperioden befürworten werden, wenn sie sich von ihr benachteiligt fühlen. Sinn der Verfassungsregein ist es aber gerade, die subjektiv empfundenen Ungerechtigkeiten ex ante auszuklammern ${ }^{3)}$, um überhaupt eine Basis für einstimmige Entscheidungen zu erreichen. Wie bereits betont, ist es natürlich bei Kenntnis der individuellen situation in den Folgeperioden normalerweise nicht mehr möglich, konsensfähige

1) Dies entspricht auch der Kernaussage bei Rawls (1971), Kap. 3, vgl. Hochmann (1974), S. 322

2) Ein anderes Ergebnis mag sich unter Berücksichtigung risikofreudiger Individuen ergeben. Bei entsprechender Risikofreudigkeit könnten diese Individuen einen gewissen Grad der Ausbeutung von Minderheiten durch Mehrheiten befürworten. Im folgenden soll jedoch von dieser Möglichkeit abstrahiert werden. Vgl. dazu West/Corke (1980)

3) Nach Hayek besteht das Grundprinzip des ökonomischen Spiels darin, daß nur das Verhalten der Spieler, nicht aber das Ergebnis gerecht sein kann. Hayek (1981), S. $102 \mathrm{ff} . \mathrm{Vgl}$. im Gegensatz dazu die Verteilungskriterien der OT, durch die bestimmte, als gerecht bezeichnete Verteilungsergebnisse angestrebt werden. 
Steuerreformvorschläge zu unterbreiten ${ }^{1}$ ).

VIII.1.3. Verfassungsregeln und individuelle Freiheit

Verfassungen sollten unter normativen Gesichtspunkten durch einen breiten Konsens oder durch Einstimmigkeit gekennzeichnet sein. Sie genügen deshalb im Zeitpunkt ihrer Verabschiedung auch dem Postulat individueller Freiheit ${ }^{2}$. Die Konzeption einer Steuerverfassung hat somit eine breitere philosophische Basis. Es sollen fiskalische Regeln festgelegt werden, die allgemeine Zustimmung finden, daher individuell gerecht sind und im laufenden politischen ProzeB freiheitswidrige obergriffe von Institutionen oder anderen Machtpotentialen verhindern.

Potentielle WohlfahrtseinbuBen im laufenden politischen ProzeB können somit auch als Kosten für zusätzliche Freiheitsgarantien interpretiert werden.

\section{VIII.2. Konstitutionelle Steuerregeln für die Demokratie}

Im folgenden sollen einige der bedeutendsten Verfassungsregeln diskutiert werden, die die Unsystematik des steuerpolitischen Willensbildungsprozesses in der Demokratie zumindest in Teilbereichen verhindern sollen. Mit ihrer Integration in eine Steuerverfassung erhofft man sich primär, daß die finanzpolitischen Entscheidungsträger zur konsequenten Verfolgung von Effizienz- und Verteilungszielen gezwungen werden.

1) $\mathrm{Zu}$ bedenken ist allerdings, daß auch schon auf der konstitutionellen Ebene die Risikoeinstellung sowie die moralischen und ethischen Werte der Individuen so stark divergieren können, daß keine einstimmigen Beschlüsse möglich werden. Vgl. dazu die divergierenden Gerechtigkeitspostulate bei Rawls (1971) und Brennan/Buchanan (1980), die auf der konstitutionellen Ebene abgeleitet werden.

2) Vgl. Buchanan (1975), S. 17 f. Die individuelle Freiheit ist gewährleistet, weil analog zum idealen Tauschprozeß am Markt niemand gezwungen wird, einer Regel zuzustimmen. 
Gleichzeitig wirken sie als Begrenzungsregeln gegen zu hohe Staatsaktivitäten.

VIII.2.1. Die Wahl der Steuerbemessungsgrundlage

Die Wirkung der Begrenzung der Steuerbemessungsgrundlage auf das maximale Steueraufkommen wurde bereits in Abschnitt $V$ diskutiert. Auch unter Berücksichtigung der Unvollkommenheiten des demokratischen Willensbildungsprozesses wäre die verfassungsrechtliche Fixierung der Steuerbemessungsgrundlage wünschenswert. Nur sie bietet einen Schutz vor wahltaktischen Manipulationen, welche dazu dienen sollen, die Steuerbemessungsgrundlage der bevorzugten Wählergruppen einzuschränken und diejenigen der benachteiligten Gruppen auszuweiten. Im Gegensatz zur Analyse des Leviathan-Modells kann eine quantitative Begrenzung der Steuerbemessungsgrundlage in der Demokratie nicht befürwortet werden, da der Fiskus nur in Ausnahmefällen maximale Steuersätze erhebt. Aus diesem Grunde kann auch die Aussage von Brennan/Buchanan, die Steuerbemessungsgrundlage könne ohne zusätzliche Mehrbelastung eingeschränkt werden, nicht mehr aufrechterhalten werden. Tatsächlich würde eine Begrenzung der Steuerbemessungsgrundlage in der Demokratie, im Gegensatz zum Leviathan-Mode11, nicht zu einem niedrigeren Steueraufkommen führen, sondern zwangsläufig zu höheren Steuersätzen in den besteuerbaren Bereichen. Damit wäre jedoch eine überproportional steigende Mehrbelastung der Zensiten verbunden ${ }^{1)}$. Begrenzungen der Steuerbemessungsgrundlage sind daher nur insoweit zu empfehlen, als nicht marktmäßig bewertetes potentielles Einkommen ${ }^{2}$ ) von der Besteuerung

1) Vg1. dazu auch Folkers (1983a), S. 54. Abgesehen von der wachsenden Ineffizienz würden auch auf konstitutioneiller Ebene nur solche Begrenzungen der Steuerbemessungsgrundlage zu erwarten sein, die den Rahmen traditioneller Gerechtigkeitskriterien nicht sprengen und somit keinen sozialen Zuindstoff in den Folgeperioden liefern.

2) Vgl. Brennan/Buchanan (1980), S. $39 \mathrm{ff}$. 
ausgenommen werden sollte. Im Zusammenhang mit der Einkommensbesteuerung impliziert dies beispielsweise, daB die Bemessungsgrundlage entsprechend den Ideen von Simons/Schanz ${ }^{1}$ ausgestaltet werden könnte ${ }^{2)}$ und durch eine allgemeine Verbrauchsteuer zu ergänzen wäre ${ }^{3)}$.

In Anbetracht demokratischer Staatswesen, die keine konsequente Maximierungsstrategie verfolgen, sollte prozeduralen Verfassungsregeln der Vorzug gewährt werden.

\section{VIII.2.2. Restriktionen für Steuersätze und Steueraufkommen}

Die Wirkung von Steuersatz-bzw. Steueraufkommensrestriktionen im Leviathan-Modell wurde bereits in Abschnitt $V$ diskutiert. Angesichts der Unsystematik der aktuellen Steuerpoltik sind einzelne Steuersatzrestriktionen jedoch nur bedingt hilfreich. Denn innerhalb ihrer Grenzen sind wahltaktische Manipulationen der Steuersätze durchaus erlaubt. Um dieses Problem zu umgehen, müßten nicht Höchstgrenzen, sondern die Steueraufkommen bzw. Steuersätze für die einzelnen Bevölkerungsgruppen fixiert werden. Dieses Verfahren erscheint jedoch zu starr und willkürlich, da Anderungen der ökonomischen Daten in den Folgeperioden (z.B. Nachfrageverhalten) unberücksichtigt bleiben. Aus diesem Grunde sollte allenfalls über Höchstgrenzen für die wichtigsten Steuerarten (z.B. Einkommensteuer, allgeme ine Verbrauchsteuer, Vermögen-oder Körperschaftsteuer) nachgedacht werden, um eine beliebige Steuerlastumverteilung zwischen den Wählergruppen zu verhindern. Jedoch

1) Vgl. zur ausführlichen Interpretation einer derartigen Steuerbemessungsgrundlage Hettich (1979), S. 1 ff.

2) Vgl. Buchanan/Tullock (1974), S. 164

3) Damit ist auch ausgeschlossen, daB Teile des Einkommens Z.B. von Staatsbediensteten aus der Bemessungsgrundlage ausgeklammert werden. Zwar würde dadurch das maximale Steueraufkommen gesenkt werden, aber Individuen, die keine Amter bekleiden, hätten in allen Folgeperioden erhebliche Nachteile. Der Konflikt mit ethisch begründeten traditionellen Postulaten horizontaler und vertikaler Gerechtigkeit ist evident. 
würde "ohne zusätzliche Regeln für die Bemessungsgrundlage [...] eine Senkung des Steueraufkommens wenig wahrscheinlich"1). offenbar sind die einzelnen quantitativen Begrenzungsregeln nur bedingt gegen die Unvollkommenheiten des demokratischen Willensbildungsprozesses wirksam ${ }^{2}$ ). Geeigneter erscheinen die im folgenden diskutierten prozeduralen Beschränkungen zur Regulierung staatlicher Steuermacht.

\section{VIII.2.3. Die Forderung nach horizontaler Gerechtigkeit}

Das Postulat der horizontalen Gerechtigkeit ist in vielen Verfassungen niedergeschrieben ${ }^{3)}$. Durch das Gebot der steuerlichen Gleichbehandlung von Personen in der gleichen ökonomischen Situation wird eine rein wahltaktische Umverteilungspolitik - zumindest zwischen "gleichen" Individuen - ausgeschlossen. Außerdem wird dadurch die theoretische Möglichkeit der perfekten Ausbeutung der Individuen verhindert ${ }^{4}$ ). Das für die finanzpolitische Praxis relevante Problem besteht in der Definition ökonomisch gleicher Situationen ${ }^{5}$ ). Um hier keine Interpretationsspielräume entstehen zu lassen, die politisch ausgenützt werden könnten, sollte jedoch in den Steuerverfassungen zusätzlich festgelegt werden, welche objektiv meßbaren ökonomischen Größen die wirtschaftliche Situ-

1) Folkers (1983a), S. 55. Vgl. auch Buchanan (1979), S. 356

2) Vgl. im Zusammenhang mit Proposition 13 in Kalifornien Z.B. Folkers (1983a), S. $132 \mathrm{ff}$., Shapiro/Sonstelie (1982). S. 190 kommen zu dem Schluß: "Thus not only is Leviathan less constrained than in the past, he is wealthier as wel1". Vgl. auch Bails (1982) and Courant/Rubinfeld (1981), insbesondere S. 114

3) Vgl. dazu Abschnitt VII.1.2. Die Forderung nach horizontaler Gleichmäßigkeit der Besteuerung entspringt z.B. in der Bundesrepublik Deutschland dem Art. 3, Abs. 1 GG. Siehe dazu Stern (1980), S. 1109 f.

4) $\mathrm{Vgl}$. dazu die Analyse in Kap. $V$ dieser Arbeit.

5) Vgl. zur Kritik an der gegenwärtigen Interpretation nach dem wirtschaftlichen Leistungsfähigkeitsprinzip Folkers (1984), S. 501 
ation eines Steuerzahlers definieren. Nichtökonomische Faktoren wie Rasse oder Religion, bleiben dabei außer acht. Aber auch die empirische Erfassung individueller Präferenzen und Fähigkeiten sollte prinzipiell untersagt werden, da diese Daten in den Händen eines Leviathan leicht miBbraucht werden könnten ${ }^{1)}$. Die Frage, ob Einkommen, Ausgaben, Vermögen usw. als Indikatoren für die Bestimmung der ökonomischen Situation der Zensiten herangezogen werden sollten, kann hier nicht im einzelnen erörtert werden ${ }^{2}$ ). Sofern man jedoch entsprechend den traditionellen Gerechtigkeitskriterien fordert, daß die Steuerzahllast gleicher Individuen näherungsweise ihren NutzeneinbuBen entsprechen sol1, wäre bei der konstitutionellen Wahl dieser Indikatoren, die gleichzeitig die Steuerbemessungsgrundlage darstellen, darauf zu achten, daB sie möglichst gut mit den individuellen Nutzenniveaus korreieren.

VIII.2.4. Das Gleichheitsprinzip von Hayek oder konstitutionelle Regeln für Steuerstrukturen

Restriktionen der Steuersatzstrukturen begrenzen nicht nur das Steueraufkommen ${ }^{3)}$, sie verhindern gleichzeitig eine beliebige Umstrukturierung des Steuersystems aus politischer Opportunität, wie es die Analyse des politischen Willensbildungsprozesses befürchten 1 äßt.

Nach Hayek ${ }^{4}$ ) impliziert die Gleichheit vor dem Gesetz auch Gleichheit in der Steuergesetzaebung. Einheitliche Steuern,

1) Vgl. Abschnitt VI.4. dieser Arbeit

2) Vgl. zur Diskussion dieser Problematik Z.B. Musgrave/Musgrave/Kullmer (1979). S. $30 \mathrm{ff}$. und Musgrave (1976)

3) Vgl. dazu Brennan/Buchanan (1980), S. $190 \mathrm{ff}$. und die Analyse Abschnitt V.3.2.

4) Vgl. Hayek (1971), S. $388 \mathrm{ff}$. 
insbesondere eine proportionale Einkommensteuer, entsprechen diesem Grundsatz, weil sie im Gegensatz zu einer progressiven Einkommensteuer keine Diskriminierung von Minderheiten durch Mehrheiten darstellen ${ }^{1}$ ). Nach Hayek sind progressive Steuersätze kennzeichnend für die willkürliche Anwendung politischer Macht, weil Mehrheiten, nur weil sie Mehrheiten sind, Minderheiten eine beliebige Steuerregel (und damit Steuerlasten) auferlegen können, die sie auf sich selbst nicht anwenden wollen ${ }^{2}$. Hayek sieht in dieser Form der Diskriminierung "eine Durchbrechung eines Prinzips, das viel fundamentaler ist als die Demokratie selbst, nämlich ein Prinzip, auf dem die Berechtigung der Demokratie beruht" ${ }^{3)}$. Aufgrund dieser Gefahren ${ }^{4}$ plädiert Hayek für eine proportionale Besteuerung, die als Grundregel (Verfassungsregel) ${ }^{5}$ ) gleicher Besteuerung aller Individuen in demokratischen Systemen zu verankern sei, wenngleich er grundsätzlich von der Funktionsfähigkeit demokratischer systeme überzeugt zu sein scheint ${ }^{6}$ ). Wie gezeigt wurde, erlaubt eine einheitliche Besteuerung in Form von proportionalen Einkommensteuern oder gleichen Steuersätzen

1) Die Forderung nach einem proportionalen Einkommensteuertarif (flat rate tax) mit Freibetrag oder mit negativer Einkommensteuer, Friedman (1971), Kap. 12. Almsick (1981) steht in jüngster Vergangenheit, wenngleich mit z.T. anderen Argumenten immer mehr im Mittelpunkt der Diskussion. Vgl. Wildavsky (1981), Hall/Rabushka (1983). Eine empirische Studie zur Wahl einer optimalen Flat-Rate Income Tax wird von Slemrod/ Yitzhaki (1983) durchgeführt.

2) Vgl. auch Buchanan/Flowers (1980), S. 183

3) Hayek (1971), S. 398

4) $\mathrm{Zu}$ weiteren Argumenten für eine proportionale Einkommensteuer siehe Hayek (1971), S. $387 \mathrm{ff}$.

5) Diese Interpretation steckt implizit in den Ausführungen von Hayek (1971), aber auch in den Erläuterungen zur Bedeutung von Regeln. Vgl. Hayek (1981), S. $23 \mathrm{ff}$.

6) Vg1. dazu Hayek (1971), S. 397 und zur Gegenposition Buchanan (1979), S. 349 
auf alle Güter, kein optimiertes Steuersystem im Sinne eines Leviathan und könnte bei gleichzeitiger Beseitigung der vielfältigen Steuerprivilegien einen wichtigen Beitrag zur systematisierung des steuerpolitischen willensbildungsprozesses leisten ${ }^{1}$ ).

Ferner wird die Möglichkeit der Umstrukturierung von Steuerlasten verringert. In bezug auf eine allgemeine Verbrauchsbesteuerung scheint dieser Vorschlag von besonderem Interesse. Dagegen ist die politische Akzeptanz einer proportionalen Einkommensteuer ${ }^{2}$ ) gering, da ein progressiver Tarif "nach wie vor als grundlegender Bestandteil der Gerechtigkeitsvorstellung einer Mehrheit der Steuerzahler gelten muB" ${ }^{3}$ ).

Aus konstitutioneller Perspektive ist die Ablehnung einer 1 inearen Einkommensteuer unproblematisch, denn wie die vorangegangene Analyse zeigt, sind auch progressive Steuerstrukturen mit der Logik einer Steuerverfassung vereinbar ${ }^{4}$ ). Welcher Progressionstarif für eine Einkommensteuer hinter dem Schleier der Unwissenheit gewählt wirde, bleibt aufgrund des Arrow-Paradoxons ein ungelöstes Problem. Jedoch sollten die in Betracht gezogenen Progressionstypen mit einem verfassungsmäßig geschützten maximalen Grenzsteuersatz und einem Freibetrag ausgestattet werden ${ }^{5)}$, so daB im Einklang mit dem Gebot der horizontalen Gerechtigkeit die Ausbeutung von Minderheiten und die steuerpolitische Willkür minimiert werden. Diese Restriktionen hätten außerdem den Vorteil, daß ein gewisser

1) Vgl. Z.B. Buchanan/Brennan (1980), S. 33 ff. und Abschnitt VII.2.5. dieser Arbeit

.2) Eine solche lineare Einkommensteuer wird gegenwärtig z.B. von Wildavsky (1981), S. 611 f. gefordert.

3) Folkers (1983a), S. 42

4) Vgl. Brennan/Buchanan (1980), Kap. 3, bzw. Abschnitt V.3.2. dieser Arbeit

5) Vgl. dazu auch Abschnitt V.3.4. dieser Arbeit 
Handlungsspielraum der politischen Akteure bestehen bleibt. Die Umsetzung des Mehrheitswillens innerhalb tolerabler Grenzen ist ebenso ein Wesensmerkmal demokratischer Verfassungen wie der Schutz von Minderheiten. Zu diesem Zweck ist aber eine gewisse Flexibilität der Steuergesetzgebung erforderlich.

\section{VIII.2.5. Die Integration von Steuer- und Ausgabenentscheidungen}

Eine der wirkungsvollsten prozeduralen fiskalischen Beschränkungen, die den demokratischen Willensbildungsprozeß möglichst eng mit den Wählerwünschen verknüpfen soll, stammt von Wicksel11). Sein Vorschlag zielt einerseits auf die Transparenz der budgetären Entscheidungsprozesse und andererseits auf den Schutz von Minderheiten ab. Diese Aspekte sind für zielorientierte Steuerreformen von ausschlaggebender Bedeutung. Die Transparenz der budgetären Entscheidungen wird durch die Integration von Steuer- und Ausgabenentscheidungen ${ }^{2}$ ) erreicht. Nur bei gleichzeitiger Betrachtung von spezifischen Steuer- und Ausgabenkategorien werden die Kosten der öffentlichen Leistungserstellung offenkundig, so daß sich ein auf objektiven Daten basierendes Wählervotum herausbilden kann.

Wicksell fordert, daß die Erhöhung spezifischer Budgetanteile mit der Anhebung spezifischer Steuern und die Einführung neuer Ausgaben mit neuen Steuern verknüpft werden sollten. Im Idealfall werden nur diejenigen Steuer-Ausgaben-Pakete gesetzlich verankert, die von der Bevölkerung einstimmig beschlossen wurden.

Der Schutz der Minderheiten wird also dadurch gewährleistet, daB Budgetänderungen nur einstimmig beschlossen werden dürfen.

1) Vgl. Wicksell (1896), S. 115. Zur Interpretation seiner Vorschläge siehe Musgrave (1974), S. $105 \mathrm{f}$. und Buchanan (1976a), S. $21 \mathrm{ff}$.

2) Vgl. auch Brennan/Buchanan (1930), S. 154 und Folkers (1983a), S. 33 ff. 
Die politische Praktikabilität erzwingt jedoch ein System relativer Einstimmigkeit ${ }^{1}$ ) (drei Viertel, füf Sechstel o.ä.), weshalb der Minderheitenschutz nicht mehr absolut gewährleistet ist. Den Vorzügen der Wicksell'schen Idee steht aber auch der Vorwurf gegenüber, sie führe zu einer Entscheidungsunfähigkeit der Demokratie bzw. zu einer inaktiven Politik2). Sicherlich dürten einer Anderung der Umverteilungspolitik gegen den Willen einer Minderheit enge Grenzen gesetzt sein ${ }^{3}$ ). Die Verfechter konstitutioneller Steuerregeln sehen deren Vorzüge gerade darin, daß bereits auf konstitutioneller Ebene das Aufkommen spezifischer steuern für konkretisierte Verteilungszwecke vorgegeben wird. Dadurch würde auch die Transparenz der staatlichen Umverteilungspolitik erhöht und die Gefahr gesellschaftlicher Konflikte gemindert ${ }^{4}$ ).

Die geringe Resonanz, die der Wicksell'sche Entwurf für budgetäre Entscheidungsprozesse gefunden hat, mag einerseits auf der Furcht der Politiker beruhen, die ihre Handlungspielräume zu sehr eingeengt sehen, andererseits gibt es aber auch erhebliche Zweifel an der Praktikabilität qualifizierter Mehrheitsbeschlüsse im politischen Alltag. Nicht zuletzt aufgrund der hohen Entscheidungskosten, die das Wicksell'sche System relativer Einstimmigkeit im laufenden politischen ProzeB verursacht, scheint es eher ein theoretisch interessantes Konzept als eine operationale Alternative zu den gegenwärtig in der finanzwirtschaftlichen Literatur diskutierten konstitutionellen Grenzen der Besteuerung zu sein.

1) Vgl. Wicksell (1896), S. 116 und 122

2) $\mathrm{Vgl}$. Musgrave (1974), S. 105

3) Im Gegensatz zu den Entscheidungen auf konstitutioneller Ebene ist den einzelnen Steuerzahlern bei diesen Abstimmungsprozessen ihre eigene Position sehr wohl bekannt.

4) Vgl. Buchanan (1963), S. 457 ff., Buchanan/Flowers (1980), S. 109 oder Musgrave/Musgrave (1984), S. 229 
VIII.2.6. Das Aquivalenzprinzip

Zur Zähmung des Leviathansund zur Eindämmung unsystematischer Steuer- und Ausgabenentscheidungen ist das Aquivalenzprinzip, das in seiner idealen Form Marktprozesse zu simulieren sucht, grundsätzlich geeignet.

Es schafft eine Verbindung zwischen Ausgaben und Einnahmen, die zu gröBerer Rationalität der individuellen und staatichen Entscheidungsprozesse führt. Aufgrund seiner individual istischen Ausrichtung verhindert das Aquivalenzprinzip die Ausbeutung einzelner Individuen. Dies gilt insbesondere bei der Bereitstellung privater Güter durch die öffentliche Hand. Probleme bei der Anwendung des Prinzips treten dann auf, wenn der Staat öffentliche Güter bereitstellt, welche von den Zensiten unterschiedlich bewertet werden. Im Idealfall müBten die Steuerpreise für diese Güter entsprechend den differierenden Grenzbewertungen variieren, um die individuellen Haushaltsoptima zu garantieren. Bisher gibt es jedoch keine praktikablen Verfahren zur Präferenzoffenbarung, so daB eine umfassende Anwendung des Aquivalenzprinzips wegen des free-rider-Verhaltens der Steuerzahler und hoher Transaktionskosten äußerst schwierig ist. Außerdem ist es für Verteilungs- und Stabilisierungszwecke wenig geeignet ${ }^{1)}$. Allerdings sollte es überall dort zur Anwendung kommen, wo eine marktähnliche Versorgung der Steuerzahler erfolgt. Der Vorteil einer solchen "Gebuihrenlösung" liegt offensichtlich vor allem in der flexiblen Anpassung staatlicher Güter und Dienste an die Wählerpräferenzen.

1) Zu weiterer Kritik am Aquivalenzprinzip siehe z.B. Neumark (1970), S. 43 ff., Frey (1976) oder Buchanan (1969), S. 25. 
VIII.2.7. Die Zweckbindung öffentlicher Einnahmen

Wenn die direkte Gebührenerhebung nicht möglich ist, kann statt dessen auch eine Steuer auf komplementäre Güter erhoben werden. Ein bekanntes Beisoiel dafür ist die Zweckbindung von Mineralöl-oder Kraftfahrzeugsteuern für den Straßenbau. Durch diese Form der Zweckbindung kann das Aquivalenzprinzip imitiert und, bei geeigneter Wahl des Steuersatzes, eine effiziente Form der Steuererhebung erreicht werden; sie eignet sich unter bestimmten Voraussetzungen auch zur Begrenzung des Steueraufkommens ${ }^{1}$ ).

Eine Zweckbindung öffentlicher Einnahmen ohne Komplementaritätsbeziehungen wirft jedoch größere Probleme auf. ${ }^{2)}$ Ein Beispiel dafür ist die property tax in den USA, die insbesondere für die Finanzierung der lokalen Schulen verwandt wird. Die Zweckbindung führt in diesem Fall nur zufällig zu einer effizienten Bereitstellung, weil die private Güternachfrage keinen Bezug zu der öffentlichen Leistungserstellung erkennen läßt. Wenn sich im Laufe der Zeit das Steueraufkommen unabhängig von den Wählerpräferenzen für die öffentliche Leistungen entwickelt, kann es somit zur Ober-oder Unterversorgung mit den entsprechenden Gütern und Diensten kommen. Ein weiterer Nachteil besteht darin, daß nicht diejenigen zur Finanzierung herangezogen werden, die den Nutzen aus der öffentlichen Leistungserstellung beziehen. In diesen Fällen kann die Zweckbindung öffentlicher Einnahmen kaum zur Verbesserung der finanzwirtschaftlichen Ergebnisse beitragen.

1) Vọl. dazu die Ausführungen in Abschnitt V.3.6.

2) Vgl. dazu Folkers (1983a), S. 34 


\section{VIII.2.8. Der materielle Haushaltsausgleich}

Die Forderung nach materiellem Haushaltsausgleich gehört ebenfalls zu den prozeduralen Begrenzungsregeln ${ }^{1)}$. Durch das Verbot der Kreditfinanzierung ${ }^{2}$ verspricht man sich eine Verminderung der Staatstätigkeit, "da bei gegebenem Zwang zur Steuerfinanzierung von Ausgaben sämtliche Programme sehr viel sorgsamer geprüt und den Bürgern die Kosten statlicher Leistungen transparent gemacht würden, während schuldenfinanzierte Ausgaben die Illusion eines 'free lunch' entstehen ließen und eine demgemäß überhöhte Nachfrage nach öffentlichen Leistungen hervorgerufen würden"3). Derartige Beschränkungen staatlicher Ausgabentätigkeit sind gleichzeitig die Voraussetzung für einen an den Wählerpräferenzen orientierten Willenbildungsprozeß in der Demokratie.

In dieser scharfen Form weist die Forderung nach materiellem Haushaltsausgleich jedoch zwei wesentliche Mängel auf, die bei einem Verfassungsvorschlag berücksichtigt werden müBten. Erstens ist es zur Wahrung der intertemporalen Effizienz und intergenerativen Gerechtigkeit notwendig, eine pay-as-you-use Kreditfinanzierung ${ }^{4}$ ) für Investitionen vorzusehen. Dies impliziert, daß zunächst spezifiziert wird, was unter dem Be-. griff Investitionen subsumiert werden darf, und welche Nutzungsdauer diese Investitionen jeweils besitzen, so "daB bereits bei dem Beschluß über die Investitionsausgaben exakte und verbindliche Verschuldungs - und Tilgungspläne mitbeschlossen werden"5). In diesem Zusammenhang müßte beispielsweise in der Bundesrepublik Deutschland der Passus. des Art. 115 GG verschärft werden, der Ausnahmeregelungen zur Abwehr einer Störung des gesamtwirtschaftlichen Gleichgewichtes zuläßt.

1) Vgl. Brennan/Buchanan (1980), S. 203 und Folkers (1983a), S. $173 \mathrm{ff}$.

2) Vgl. zum Problem wachsender Budgetdefizite Buchanan/Wagner (1977) und Baum (1982)

3) Folkers (1983a), S. 37

4) Vgl. dazu Musgrave (1974), S. 523 f. 
Ein Vorschlag besteht darin, daß nur qualifizierte Mehrheiten ein gesamtwirtschaftliches Ungleichgewicht feststellen und ein verbindliches Verschuldungs- und Tilgungsprogramm verabschieden können ${ }^{1}$ ). Das Beispiel demonstriert, daß es in der Verfassung der Bundesrepublik Deutschland durchaus fiskalische Begrenzungsregeln gibt; ihre Mängel beruhen jedoch meist auf einem zu großen Interpretationsspielraum, der in der finanzpolitischen Praxis zu leicht miBbraucht werden kann. Vorschläge zur Anderung der Finanzverfassung, die diese Schwachstellen ausmerzen sollen, rücken seit geraumer Zeit immer mehr in den Mittelpunkt der finanzpolitischen Diskussion ${ }^{2}$.

\section{VIII.2.9. Föderalismus und Besteuerungsmacht}

In föderativen Staatswesen können eine effektive Begrenzung des Steueraufkommens und ein an den Wählerwünschen ausgerichteter Steuerreformprozeß dadurch erreicht werden, daB Steuer- und Ausgabenentscheidungen nicht nur in der Kompetenz der Zentralregierung liegen, sondern weitgehend dezentralisiert werden ${ }^{3}$ ). Dies impliziert eine "Seperation der unterschiedlichen fiskalischen Funktionen und der einzelnen Ebenen des föderativen Staates"4). In diesem Zusammenhang müßte vor allem der Anziehungskraft größerer Etats entgegengewirkt werden ${ }^{5)}$. Der Wettbewerb zwischen den untergeordneten Gebietskörperschaften verhindert die Ausbeutung der Zensiten und bewirkt eine effizientere Budgetgestaltung, weil den Bürgern durch das Wahlrecht ihres Wohnortes, (voting with the feet) ${ }^{6}$ ), eine permanente Kontrolle

1) Vgl. Folkers (1983a), S. 37/38 und Baum (1982), S. $192 \mathrm{f}$.

2) Vgl. dazu Baum (1982), S. $172 \mathrm{ff}$.

3) $\mathrm{Vgl}$. dazu auch Brennan/Buchanan (1980), S. $173 \mathrm{ff}$. und Folkers (1983a), S. $35 \mathrm{f}$.

4) Folkers (1983a), S. 35

5) Diese Entwicklung ist unter dem Begriff Popitz'sches Gesetz bekannt.

6) Art. 109 GG gebietet die Haushaltstrennung von Bund und Ländern. Welche Aufgaben vom Bund bzw. von den Ländern wahrgenommen werden sollten, wird jedoch nicht festgelegt. 
der föderativen Fiskalgewalten ermöglicht wird ${ }^{1}$.

Eine Verbesserung der steuerpolitischen Willensbildung könnte auch dadurch erreicht werden, daß die Steuergesetzgebungshoheit für alle wichtigen Steuern nur vom Bundesparlament und den Länderparlamenten gleichzeitig wahrgenommen werden kann. Das bedeutet, daß sämtliche Ebenen des föderativen Staatswesens einer Steuerrechtsänderung zustimmen müßten, um so einen Kompromiß sicherzustellen, der keine Region benachteiligt. In der Bundesrepublik Deutschland hätte dies eine entsprechende Änderung des Art. 105 GG zur Folge. Da die Parlamente regelmäßig mit unterschiedlichen Mehrheiten besetzt sind, werden kurzfristige Steuergesetzänderungen im Interesse einzelner Gruppen erschwert und die Gefahr der Ausbeutung von Minderheiten durch Mehrheiten drastisch vermindert. Allerdings kann auch dieser Empfehlung der Vorwurf mangelnder Flexibilität gemacht werden, da sich notwendige Reformmaßnahmen durch überlange Verhandlungen zwischen den Gebietskörperschaften erheblich verzögern können.

\section{VIII.2.10. Sunset Legislation}

Die Sunset Legislation ${ }^{2)}$ wird meist auf der Ausgabenseite des Budgets wirksam. Sie weist einzelnen Ausgabenkategorien bestimmte Laufzeiten zu, nach deren Ablauf das Parlament uiber eine etwaige Verlängerung neu abstimmen muß. Dadurch wird die Volksvertretung immer wieder gezwungen, über die ZweckmäBigkeit alter Programme zu befinden. Das Konzept der "sunset legislation" ist somit geeignet, den demokratischen willensbildungsprozeß transparenter und systematischer zu gestalten. Es sollte deshalb auch vermehrt im Steuerreformprozeß eingesetzt werden, wenn es darum geht, spezifische steuervorteile zu gewähren. Die Abschaffung von Steuervorteilen würde politisch erheblich erleichtert, wenn sie nach einer gewissen Laufzeit (z.B. einer Legislaturperiode) automatisch wegfielen

1) Vgl. zu einer ausführlichen Diskussion dieses Konzeptes Tiebout (1956), S. $416 \mathrm{ff}$. oder Boadway (1979), S. $405 \mathrm{ff}$.

2) Vgl. dazu Z.B. Folkers (1983a), S. 39 f. oder Langner (1983) S. 261 ff. 
und nur durch einen expliziten Parlamentsbeschluß verlängert werden könnten.

\section{VIII.2.11. Zur Notwendigkeit weiterer Verfassungsregeln}

In den vorangegangenen Abschnitten wurde bereits ausgefüht, da $B$ in den meisten Demokratien verfassungsrechtliche Schranken der Fiskalgewalt bestehen. Offensichtlich reichen diese Beschränkungen nicht aus, um langfristig rationale Steuerstrukturen zu gewährleisten. Die vorliegenden fiskalischen Verfassungsregeln bilden die Grundlage für die weitere Diskussion zur Verbesserung des Willensbildungsprozesses. Sie beinhalten keinesfalls die Gesamtheit aller denkbaren Lösungsvorschläge. Vielmehr sollten einige Regeln für die bisher mangelhafte "Ordnungspolitik für den öffentlichen Sektor"1) konkretisiert werden; gleichzeitig sollte ein Anstoß einer weiteren Entwicklung neuer, ergänzender Steuerregeln gegeben werden. Die dringendste Reform ist die Schaffung neuer bzw. die Reform bestehender verfassungsrechtlicher Steuerregeln, nicht die Anderung einiger Steuergesetze ${ }^{2}$ ). Der steuerpolitische WillensbildungsprozeB selbst, d.h. die Regeln, die zur Entscheidungsfindung führen, sollten somit im Mittelpunkt der steuerreformdebatte stehen.

Neben den bisher erörterten Vorschlägen, die in der Literatur bereits seit längerem diskutiert werden, wird im folgenden auf der Basis der positiven Analyse des demokratischen Steuerreformprozesses eine Verfassungsregel entwickelt, die einer der gravierendsten Schwächen der aktuellen Steuerpolitik - nämlich der unkontrollierten Steuerprivilegvergabe - entgegenwirken sol1.

1) Vgl. Folkers (1983a), S. 58

2) Reformvorschläge im Sinne der OT würden ohnehin nur kurzfristig Beachtung finden, sofern sie überhaupt politisch durchsetzbar wären. 
Wie bereits analysiert, stellen die Dynamik, die Vielzahl und das Beharrunqsvermögen ${ }^{1)}$ der bis heute kumulierten steuerprivilegien wesentliche Ursachen für die Unsystematik ${ }^{2}$ aktueller Steuersysteme dar. So stieg der Anteil der Subventionen an der Bruttowertschöpfung, die sogenannte Subventionsrate, von 4,9\% im Jahre 1966 auf 6,5\% im Jahre 19783). Ahn1 iches gilt in den USA, wo in der Einkommensteuergesetzgebung annähernd $111 \mathrm{Mrd}$. Dollar an Steuervergünstigungen gewährt werden, so daß es nicht wundert, daß Steuervergünstigungen im Blickpunkt der wissenschaftlichen und politischen Reformdiskussion stehen ${ }^{4}$ ).

1) Ein gruppenspezifisches Beispiel für das harte Ringen um traditionelle Steuerverguinstigungen liefern die Aktivitäten des Hochschulverbandes gegen die Abschaffung des $\S 34$ Absatz 4 Einkommensteuergesetz. Vgl. dazu Mitteilungen des Hochschulverbandes 6 (1980), S. $287 \mathrm{ff}$. Weitere Beispiele dieser Art lassen sich in den permanenten Debatten um den Subventionsabbau ohne weiteres finden. $\mathrm{Vgl}$. den Neunten Subventionsbericht der Bundesregierung vom 6. September 1984 und die Bulletins des Presse- und Informationsamtes der Bundesregierung 85 (1983), S. 782 und 86 (1983), S. 798.

2) Für Subventionen ist ein hohes Maß an Willkür und an fehlender Regelgebundenheit charakteristisch, vgl. Andel (1977), S. 503

3) Die Zahlen beruhen auf Berechnungen des HWWA-Institutes (1980), Zitat nach Weilepp (1982), S. 329. Die offiziellen Zahlen der Bundesregierung liegen niedriger (ca. $2 \%$ am BSP). Das tatsächliche Ausmaß der Subventionen wird in den offiziellen Subventionsberichten jedoch nicht ausgewiesen, weil wesentliche Hilfen an staatliche Unternehmen (Bundesbahn, Bundespost), ERP-Darlehen oder EG-Hilfen dort nicht aufgeführt werden. Alle Sozialleistungen, Steuerermäßigungen und Sachleistungen addieren sich sogar zu 31 \% am BSP. Vgl. Lampert (1982), S. 475

4) Die "tax expenditures" insgesamt sind in den USA von ca. 50 Mrd. Dollar 1968 auf 170 Mrd. Dollar 1980 gestiegen. Siehe Alt (1983), S. 211. Ebenso wird die Bedeutung von Steuervergünstigungen in der Bundesrepublik Deutschland in vielen Publikationen verdeutlicht. $\mathrm{Vgl}$. dazu die Subventionsberichte, den Bericht der Transfer-Enquête-Kommission (1981) und die Finanzberichte des BMF. 
In Abschnitt VIII.2.5. wurde gezeigt, daB die volkswirtschaftlichen Verluste aus dem Kampf um Subventionen normalerweise viel zu gering eingeschätzt werden, und die Verteilungsgewinne aus den erkämpften Steuervergünstigungen oft nur von kurzer Dauer sein werden. Daher muB dieser ProzeB im Interesse der Wähler umstrukturiert und unabhängig von zyklischen Mehrheiten kontrollierbar sein.

VIII.3. Die Forderung nach konstitutionellen Grenzen für die Steuerprivilegpolitik

Die Vorzüge konstitutioneller Regeln lassen sich anhand der Steuerprivilegpolitik gut illustrieren. Um eine rationale Steuerprivilegpolitik zu gewährleisten, sollte die Vergabe öffentlicher Hilfen an die Verfassungsregel gebunden werden.

Die folgende Verfassungsregel sollte lediglich als verbesserungsfähige Fallstudie verstanden werden. Ihre Aufgabe besteht vorrangig darin, im Einklang mit anderen Steuergesetzen sowie nichtfiskalischen Restriktionen und den zuvor erörterten fiskalischen Verfassungsregeln eine normkonsistente Steuerpolitik zu forcieren. Sie kann nur eine Ergänzung, kein Ersatz für einen funktionierenden Parteienwettbewerb oder sonstige Kontrollinstrumente staticher Macht sein ${ }^{1)}$. Die folgende Diskussion der Steuerprivilegregel soll im Zusammenhang mit

1) Vgl. dazu Brennan/Buchanan (1980), Kap. 2 
einem integrierten Einsatz weiterer Verfassungsregeln unter Berücksichtigung der positiven Analyse demokratischer Steuerreformprozesse erfolgen.

\section{VIII.3.1. Eine Verfassungsregel für Steuerprivilegien}

Um die bisherigen Ausführungen zu konkretisieren, wird auf der Basis einer umfassenden Steuerbemessungsgrundlage und des Prinzips der GleichmäBigkeit der Besteuerung ${ }^{1)}$ folgende Verfassungsregel für sinnvoll erachtet. Sie möge vier Jahre nach ihrer Bekanntgabe in Kraft treten.

(1) Die Vergabe von Steuerprivilegien wird nur noch dann rechtskräftig, wenn die Parlamente mit zwei Dritteln ihrer Mitglieder zustimmen. Nach Inkrafttreten dieser Verfassungsregel ist über alle Steuerprivilegien spätestens nach jeweils vier Jahren erneut abzustimmen. Wird in dieser Abstimmung die Zweidrittelmehrheit verfehlt, sind die Steuerprivilegien nach einer obergangszeit von maximal zwei Jahren zu streichen ${ }^{2}$ ).

(2) Ausnahmen von dieser Regelung sind in einem exakt spezifizierten, operationalen Steuerprivilegkatalog ${ }^{3)}$ niederzulegen. Anderungen des Kataloges sind nur mit Zustimmung von zwei Dritteln der Parlamentsmitglieder gültig. Der Katalog enthält die mit einfacher Mehrheit zulässigen Steuerprivilegformen, regelt deren Zuordnung zu bestimmten Allokations- sowie Verteilungs-

1) Vgl. dazu die Analyse der Allokations- und Verteilungswirkungen von Steuerprivilegien in Abschnitt VII.2.5.

2) Vgl. dazu Abschnitt VIII.2.10. sunset legislation

3) Vgl. dazu die ersten Bemühungen um einen Subventionskodex zwischen den Bundesländern oder den Subventionskodex Stahl der Kommission der Europäischen Geme inschaft vom 7. August 1981; siehe Neunter Subventionsbericht der Bundesregierung, $\mathrm{S}$. 313. Vgl. zu einigen aktuellen politischen Vorschlägen Breuel (1983). 
zielen und listet jene Kriterien auf, die vor der Steuerprivilegvergabe erfüllt sein müssen. Alle hiernach gewährten Steuerprivilegien sind jeweils nach spätestens vier Jahren von der Exekutive sowie von einem Sachverständigengremium zu uiberprüfen. Werden die Voraussetzungen nicht mehr erfüllt, d.h. stimmen nicht mindestens jeweils zwei Drittel der Mitglieder beider Prüfungsinstanzen zu, sind die Steuerprivilegien nach einer ubergangszeit von maximal zwei Jahren zu streichen ${ }^{1}$ ).

\section{(3) Das Nähere regelt ein Bundesgesetz}

Dieser Verfassungsvorschlag ist als mittel-bis langfristiges Steuerreformprogramm $z u$ interpretieren. Bei seiner Realisierung muß mit einigen politischen und sachlichen Schwierigkeiten gerechnet werden. Erstere ergeben sich insbesondere aus den Widerständen der Interessengruppen gegen den zu erwartenden Abbau bestehender Steuerprivilegien. Darüber hinaus werden aber auch die Politiker nicht ohne weiteres für diese Regel stimmen, da sie ihren Handlungsspielraum einengt.

Die inhaltichen Probleme resultieren aus der Definition einer umfassenden Steuerbemessungsgrundlage und einer allgemeinen Steuerstruktur (z.B. allgemeine Verbrauchsteuer in Verbindung mit einer progressiven Einkommensteuer) bzw. aus der Abgrenzung von Steuerprivilegien. Die Scheu vor einer exakten Begriffsbestimmung ist jedoch selbst eine wesentliche Ursache für die unsystematische Steuerprivilegvergabe. Die Verschleierung konkreter Verteilungsvorstellungen und die Undurchschaubarkeit der gegenwärtigen Subventionsvergabe erschwert die Verfolgung und Kontrolle der Verteilungspolitik oder macht sie gar un-

1) Damit ist auch dieser Teil der Vergünstigungen mit einem SunsetElement versehen. 
möglich. Die Vorzüge dieser Regelung, welche im folgenden näher erläutert werden, sollten daher überzeugen können.

VIII.3.2. Vorteile einer konstitutionellen Steuerprivilegregel

Absatz (1) der Steuerprivilegregel nutzt die in diesem Kapitel erörterten Vorzüge konstitutioneller Steuerregeln im laufenden politischen Prozeß. In bezug auf Steuerprivilegien lassen sie sich wie folgt zusammenfassen:

1. Aus konstitutioneller Sicht sind gewährte Steuerprivilegien effizient und gerecht, weil sie mit relativer Einstimmigkeit verabschiedet werden. Die willkürliche Bedienung von Partikularinteressen ist nicht mehr möglich ${ }^{1}$ ), weil eine Zweidrittelmehrheit über die Subventionsvergabe befindet. Damit verschwindet auch die Dynamik der Subventionsspirale. Steuerprivilegien werden zu langfristig kalkulierbaren Rahmenbedingungen, so daB die Informations- und Anpassungskosten gegenüber der gegenwärtigen Situation erheblich gesenkt werden können.

2. Mit der Beseitigung der Komplexität des gegenwärtigen Steuersystems wird auch der verbesserten Kontrolle staatlicher Aktivitäten Rechnung getragen. Die verminderte Anzahl von Steuerprivilegien erhöht die Chance für die Beurteilung ihrer Verteilungswirkungen und macht daher eine effizientere Verfolgung des Verteilungszieles möglich.

1) Theoretisch könnte die Subventionsvergabe auch mittels Logrolling erfolgen. Jedoch würde in den Verhandlungen mit beinahe allen Anwärtern auf Steuerprivilegien klar, daß die Vergabe von Verteilungsvorteilen an alle Gruppen nur durch höhere allgemeine Steuern finanzierbar wäre und daher letztlich kaum einer Gruppe Vorteile erbringen würde. 
3. Ausnahmeregelungen von der Steuerprivilegregel können vorgenommen werden. So könnten jene Einkommenstransfers von einer permanenten oberprüfung ausgenommen werden, die zur Existenzsicherung der Individuen notwendig sind ${ }^{1)}$. Diese Fragen sollten statt dessen im Zusammenhang mit einer negativen Einkommensteuer gelöst werden ${ }^{2)}$. Ebenso könnte man sich auf konstitutioneller Ebene darauf einigen, Transfers, die zur Kompensation von psychischen und physischen Behinderungen dienen, aus einer permanenten oberprüfung herauszunehmen. Die dauerhafte Gewährung solcher Transfers wäre auch deshalb unproblematisch, weil sie an objektiv meßbaren Erscheinungen anknüpfen und aus einem konstitutionellen Versicherungsmotiv ${ }^{3}$ ) heraus individualistisch begründbar sind.

Absatz (2) der Verfassungsregel betrifft Steuerprivilegien, die mit einer Mehrheit von $50 \%$ der Parlamente verabschiedet werden können. Vor ihrer Vergabe sind sie jedoch einer strengen Prüfung zu unterwerfen, die ebenfalls von dritter seite, z.B. einem Sachverständigengremium nachvollzogen werden kann. Auch wenn die Subventionsvergabe aus Gründen der Flexibilität mit einfacher Mehrheit zu bewerkstelligen ist, sind die Prüfkriterien selbst verfassungsrechtlich verankert. Die Vorteile liegen in folgendem:

1) Vgl. Z.B. Hayek (1981), S. 122

2) In den Bereich der negativen Einkommensteuer fallen vor allem Transfers, die zur Sicherung des Existenzminimums notwendig sind. Diese Transfers werden von der Steuerprivilegregel explizit ausgenommen. Ob eine negative Einkommensteuer einer persönlichen Ausgabensteuer vorzuziehen ist, soll hier jedoch nicht diskutiert werden. Vgl. zu einem Oberblick über diese konträren Positionen Z.B. Friedman (1971), Kap. 12, Almsick (1981), Kaldor (1955) und Zumste in (1977)

3) Vgl. dazu Buchanan/Tullock (1962), S. $189 \mathrm{ff}$. oder Buchanan/Flowers $(1980)$, S. $95 \mathrm{f}$. 
1. Die Definition und Erfassung aller der Regierung zur Verfügung stehenden Steuerprivilegien führt zu einer effizienten Kontrolle der Steuerprivilegpolitik. Die insbesondere von Surrey angeprangerte Trennung von allgemeinem Steuersystem und Steuerprivilegien könnte zugunsten eines einheitlichen, rationalen Konzepts der Besteuerung überwunden werden.

2. Ein weiterer Vorteil der Steuerprivilegregel liegt darin, daß Paretoverbesserungen ohne aktuelle Kompensationszahlungen möglich sind ${ }^{1}$ ). Der Grund liegt darin, daB mit der Abschaffung von Steuerprivilegien die Steuerbemessungsgrundlage ausgeweitet wird und gleichzeitig eine Senkung der übrigen Steuersätze möglich ist ${ }^{2}$. Da von diesen MaBnahmen praktisch alle Individuen der Gesellschaft betroffen sein durften und andererseits die Allokationsverluste proportional zum Quadrat der Steuersatzsenkung abnehmen, ist zu vermuten, daß sich der überwiegende Teil der Bevölkerung besser stellen wird ${ }^{3)}$.

3. Die Existenz eines operationalen Kriterienkatalogs für die Subventionsvergabe würde den Handlungsspielraum für unsystematische Steuerprivilegien weiter einengen. In diesem Zusammenhang müBte $z . B$. fixiert werden, welche Art von Marktstörungen ( $z . B$. Wettbewerbsverzerrungen, externe Effekte, Monopole und ähnliches) und welches Ausmaß dieser Störungen gegeben sein müssen, um allokative Eingriffe zu rechtfertigen. Solange keine meBbaren, konsensfähigen Indikatoren für vermutete Allokationsverzerrungen vorliegen, durfen keine staatlichen Hilfen gewährt werden. Weiterhin wäre zu regeln,

1) Dies ist besonders wichtig, weil Steuerreformen mit schwerwiegenden Verteilungsänderungen kaum eine politische Realisierungschance haben. $\mathrm{Vgl}$. Ben-Porath/Bruno (1977), S. 303

2) Val. dazu Break/Pechman (1975), S. 135

3) Vgl. Buchanan/Brennan (1980), S. $47 \mathrm{ff}$. und Abschnitt VII.2.5. dieser Arbeit 
nach welchen einheitlichen Kriterien eine Erfolgskontrolle der gegenwärtigen Subventionen vorzunehmen ist. Die Vergabe öffentlicher Mittel muB untersagt werden, wenn keine operationalen Indikatoren existieren. Ein Beispiel für meBbare Indikatoren ist die Verminderung von Schadstoffemissionen, die aufgrund von Subventionsversprechen erfolgen ${ }^{1}$ ).

4. Mit dieser Regel bleibt die Flexibilität bei der Vergabe konsensfähiger Subventionen erhalten, weil im Einklang mit dem Subventionskatalog eine zügige Mittelvergabe erfolgen kann. Ober strittige Fragen entscheidet eine mit unabhängigen Fachleuten besetzte Schiedsstelle mit Zweidrittelmehrheit ihrer Mitglieder. Ein weiterer Vorteil der Steuerprivilegregel besteht darin, daß dem Kampf um individuelle Verteilungsvorteile der Boden weitgehend entzogen wird, so daß die erheblichen Reformkosten der Reformbeteiligten sinken.

5. Eine permanente Öberprüfung bestehender Steuerprivilegien soll der dauerhaften Existenz unbegründeter Steuergeschenke entgegenwirken. Die Regel gewährleistet, daB sinnlos gewordene Steuerprivilegien nach einer ubergangszeit von maximal zwei Jahren zwangsläufig abgeschafft werden ${ }^{2}$ ).

Da konstitutionelle Steuerregeln zukunftsorientiert sind, haben sie den Vorteil, daB sie lange vor ihrem Inkrafttreten bekannt sind und deshalb von den Individuen antizipiert wer-

1) Dies bedeutet, da $B$ die Beweislast für die Sinnhaftigkeit von Subventionen beim Empfänger liegt. Vgl. zu dieser Forderunq Z.B. Lambsdorff (1984). S. 778

2) Nach Auffassung der meisten Steuerexperten sollten die Reduktion bzw. Abschaffung von Steuerprivilegien das Hauptanliegen kommender Steuerreformen sein. Vgl. Z.B. Break/Pechman (1975), insbesondere Tabelle $6-3$, S. 128 
den können ${ }^{1)}$. Je flexibler die Antizipation, um so qeringer werden die Anpassungskosten für die Wirtschaftssubjekte sein ${ }^{2}$ ). Wichtiger aber ist, daB die einzelnen Individuen ihre zukünftige Situation nicht eindeutig kennen und daher zumindest einen gewissen Anreiz haben, effizienten und gerechten Verfassungsregeln zuzustimmen ${ }^{3 \text { ) }}$.

Unter Berücksichtigung dieser Vorzüge können die Chancen einer qualifizierten Mehrheit für die konstitutionelle Steuerprivilegregel generell positiv beurteilt werden.

1) "[...] enacting a tax change with a delayed effective date can reduce the arbitrary losses incurred by individuals who have acted in reliance upon the current laws". Feldste in (1976a), S. 99. Durch die Vorankuindigung wirkt die Reform bei den Betroffenen nicht willkürlich. Hochman (1974), S. $324 \mathrm{f}$.

2) Vgl. Feldstein (1976a), S. $98 \mathrm{f}$.

3) Ahnlich Hochman (1974), S. 322 


\section{ZUSAMMENFASSUNG}

Die Steuersysteme der entwickelten westlichen Volkswirtschaften sind in den letzten Jahrzehnten trotz der gewaltigen Fortschritte in der normativen Steuerlehre nach Meinung von Experten, Politikern und Steuerzahlern immer komplizierter, ineffizienter und ungerechter geworden. Wie in Kapitel I ausgefüht wird, sucht die Arbeit eine Antwort auf die Frage, warum eine so tiefe Kluft zwischen theoretischer Erkenntnis und steuerpolitischer Realität besteht und wie sie verringert werden kann.

Zu diesem Zweck werden in Kapitel II der Arbeit das Konzept und einige Ergebnisse der Theorie der optimalen Besteuerung (OT) vorgestellt. Im Mittelpunkt der Betrachtung stehen die OT-Regeln für eine effiziente Besteuerung, die gleichzeitig den Referenzrahmen für die weitere Untersuchung darstellen. Die Analvse zeigt, daß die spezifischen Steuerreformempfehlungen der OT im Falle vollkommener Information, vollkommenen Wettbewerbs und konstanter Produzentenpreise von der Art der Steuerbemessungsgrundlage, dem Steuerinstrumentarium und den individuellen Präferenzen abhängen. Jede Variation dieses Datenkranzes impliziert im allgemeinen Anderungen der Optimalregeln. Realistischerweise muß aber mit vielfältigen Marktverzerrungen und laufenden Anderungen der ökonomischen und politischen Rahmenbedinaungen gerechnet werden, so daß eine permanente Reform des optimalen Steuersystems erfolgen müßte. Das Streben nach optimalen Steuersystemen ist daher naturgemäB kurzfristig und, finanzpolitisch betrachtet, kaum operational.

Diese Einschätzung wird auch durch die Analyse in Kapitel III bestätigt, die zeigt, daß in der okonometrie noch viele Schätzprobleme gelöst werden müssen, bevor verläßliche 
Elastizitätswerte ermittelt werden können, die als empirische Basis für OT-Regeln brauchbar sind. Gegenwärtig ist zu befürchten, daß die Struktur der Steuersätze nicht auf ökonometrischen Parameterschätzungen beruhen, sondern durch ungetestete, willkürliche ökonometrische Hypothesen determiniert werden. Tatsächlich kann in den aktuellen Steuerreformdebatten beobachtet werden, daß die jeweiligen Reformentscheidungen oder -absichten meist nur von Vermutungen über die ökonomischen Reaktionen der Steuerzahler getragen werden.

In Kapitel IV wird diskutiert, welche Auswirkungen die Berücksichtigung von Verteilungszielen auf die Effizienzrege In hat. Die Einführung von Verteilungsnormen in Form einer sozialen Wohlfahrtsfunktion (SWF) führt, je nach Wahl der Verteilungsgewichte, zu erheblichen Modifikationen der bisher abgeleiteten Effizienzreaeln. Der Vorteil der OT besteht darin, ökonomische Wirkunaen von Steuern zu analysieren und den Konflikt zwischen Effizienz- und Gerechtiakeitszielen zu präzisieren, ohne jedoch verbindliche Lösungen für allokative und distributiv optimale steuerstrukturen begründen zu können.

Aus finanzwissenschaftlicher Sicht scheint es jedoch bedenklich, daß in der OT das Problem der politischen Entscheidungsfindung von Effizienz- und Gerechtigkeitsnormen ebenso ausgeklammert wird wie die Frage, welche staatlichen Institutionen normkonsistente Regeln durchsetzen sollen. Bleibt diese Kritik unbeantwortet, so reduziert sich die OT auf ein reines optimierungsverfahren ohne Beziehung zur finanzpolitischen Realität. Damit sind wesentliche Ursachen für die Diskrepanz zwischen OT-Regeln und aktuellem Steuersystem genannt. Davon unberührt bleibt die Bedeutung der OT als "Grammatik" für die theoretischen Implikationen alternativer steuerstrukturen. 
Anderungen von Hypothesen über die Verhaltensweise der finanzpolitischen Entscheidungsträger machen das Dilemma der OT deutlich. Unterstellt man, wie in Kapitel V, einen Leviathanstaat, d.h. einen Fiskus, der das Steueraufkommen auf Kosten der Bürger zu maximieren trachtet, so kommt man zu dem Ergebnis, daB die Struktur der Leviathanregeln mit jener eines wohlwollenden Planers im Ein-Konsumenten-Modell übereinstimmt. Brennan/Buchanan haben daraus den Schluß gezogen, daß OT-Regeln wegen der potentiellen Ausbeutungsgefahr abgelehnt werden sollten. Statt dessen seien konstitutionelle Steuerregeln zu entwickeln, die die staatliche steuergewalt derart beschränken, daß lediglich ein an den Wählerwünschen orientiertes Bündel öffentlicher Güter bereitgestellt werden kann. Am Beispiel von Begrenzungen der Steuerbemessungsgrundlagen, der Steuersätze und des Steueraufkommens werden zunächst die Funktions- und Wirkungsweise ergebnisbezogener, fiskalischer Verfassungsregeln demonstriert. Die Wahl der geeigneten Restriktionen erfolgt durch einstimmigen Beschluß der Steuerzahler auf konstitutioneller Ebene. Nach Brennan/Buchanan bedarf es somit in erster Linie der Verbesserung der bestehenden Finanzverfassungen bzw. der konstitutionellen Rahmenbedingungen, nicht der Reform einzelner Steuergesetze. Die Diskussion um Implikationen und Kritik an der konstitutionellen Steuertheorie macht deutlich, daß die Loqik und die Empfehlung quantitativer konstitutioneller Steuergrenzen entscheidend auf der Leviathanthese beruhen.

Auf den ersten Blick scheint der in den meisten Demokratien wachsende Staatsanteil die These zu erhärten; andererseits aber zeigt ein Vergleich der gegenwärtig existierenden Verbrauch- und Einkommensteuergesetze mit den Leviathan- (bzw. OT-) Regeln, die sich bei vollkommener Information und ohne politische Restriktionen des Fiskus ergeben würden, daß diese Modellspezifikation keine ausreichende Erklärung für den Stand der aktuellen Steuerstrukturen liefert. Somit stellt sich die Frage, inwieweit die Modellprämissen modifiziert werden müssen, damit die in der Realität beobachtbaren Ab- 
weichungen von aktuellen und optimierten Steuerstrukturen begründet werden können.

Zu diesem Zweck wird in Kapitel VI ein Leviathanmodell entwickelt, mit dessen Hilfe die Auswirkungen von Informationsmängeln auf die Entscheidungen der Finanzpolitiker untersucht werden. Die Reformentscheidungen hängen in diesem Falle der UngewiBheit, von dem Erwartungswert des Entscheidungsträgers und seinen Risikopräferenzen ab. Das bedeutet, daß c.p. mit der Anderung der Risikopräferenzen des Entscheidungsträgers auch die optimalen Steuerstrukturen variieren werden. Weiche Optimierungsergebnisse von staatlicher seite zu erwarten sind, hängt daher vom Informationsstand und den Präferenzen des Entscheidungsträgers ab. Diese Erkenntnis liefert gleichzeitig eine Erklärung für die vielfach als unsystematisch bezeichneten Abweichungen der aktuellen von den optimierten Steuersystemen bei vollkommener Information. Im vorliegenden Modell $1 \ddot{B}$ t sich außerdem nachweisen, daß ein risikoscheuer Leviathan normalerweise steuersätze erheben wird, die unterhalb der das Steueraufkommen maximierenden Steuersätze liegen. In diesem Zusammenhang sind Informationsdefizite seitens des Leviathan durchaus positiv zu beurteilen. Außerdem belegen die Ergebnisse, daß Datenschutzmaßnahmen als konstitutionelle Restriktion zur Begrenzung der perfekten Ausbeutungsmöglichkeiten des Leviathan notwendig und sinnvoll sind. Dennoch lassen sich in diesem Modell nicht alle unsystematischen Erscheinungen des aktuellen Steuersystems erklären. Beispielsweise sind Freibeträge in der Einkommensteuer oder sehr niedrige Steuersätze auf Grundnahrungsmittel nur unter Berücksichtigung politisch-ökonomischer Restriktionen zu erklären.

In Kapitel VII wird daher eine politisch-ökonomische Analyse gegenwärtiger Steuerreformprozesse durchgeführt. Dabei kann zunächst festgehalten werden, daß die staatlichen Entschei- 
dungsträger durch vielfältige fiskalische und nichtfiskalische konstitutionelle Restriktionen in ihren Aktivitäten begrenzt werden. Beispielsweise verhindern die Forderungen nach horizontaler Gleichbehandlung und der demokratische Parteienwettbewerb die effiziente Ausbeutung der Steuerzahler. AuBerdem scheint auch die Vorstellung von einer monolithischen Aufkommensmaximierungsfunktion des Fiskus unrealistisch, weil sich die an den steuerpolitischen Entscheidungen beteiligten Bürokraten und Politiker durch unterschiedliche Zielsetzungen leiten lassen, wobei die Interessen beider Gruppen teilweise miteinander konfligieren. Auch wenn sich die Leviathan-Hypothese nicht uneingeschränkt bestätigen läßt, kann das streben von Politikern und Bürokraten nach finanziellen Handlungsspielräumen auch empirisch als gesichert gelten. Im OT-Modell kann man zeigen, daB Steuererhöhungen ohne Minderung der Nutzenposition der Steuerzahler möglich sind. Selbst in funktionsfähigen Demokratien mit Mehrheitswahlrecht kann durch eine entsprechende Reformstrategie eine begrenzte Aus beutung von Minderheiten durch die Regierungspartei erfolgen. Der Trend zu einem systematisch überhöhten Steueraufkommen (bzw. Budget) wird weiterhin durch das Informationsmonopol der Bürokratie verstärkt. Aufgrund der bürokratischen 0ptionsfixierungsmacht können die Politiker (bzw. Wähler) nur zwischen überhöhten Budgets wählen. In welchem Umfang das aktuelle von demjenigen Steueraufkommen abweicht, das den Wählerwüschen entsprechen würde, kann empirisch jedoch nicht bestimmt werden.

Sieht man vom fiskalischen Ziel aktueller Steuerreformpolitik $a b$, so liegt offenbar eine wesentliche Schwäche in der vielfach wahltaktisch orientierten Steuergesetzgebung, die nur zufällig mit vorgegebenen Effizienz- und Verteilungszielen kompatibel sein wird. Dieser systemimmanente Mangel wird dadurch verstärkt, daß die Interessengruppen innerhalb der Legislaturperiode aktiv in den politischen willensbildungsprozeB eingreifen. 
Ausgehend von der Nutzenmaximierungshyothese wird unterstellt, daß das zentrale Interesse von Steuerzahlern darin besteht, ihre Gesamtsteuerlast zu minimieren. Die Durchsetzung dieses individuellen Steuerreformziels wird an Interessengruppen delegiert. Diese werden nicht versuchen, allgemeine Steuertarifänderungen, Begrenzungen der Steuerbemessungsgrundlagen u.ä. anzustreben, da die Reformkosten für eine allgemeine Steuerlastsenkung relativ hoch sind und das Nettoergebnis einer Reform ungewiB ist. Daher rückt die individuelle Steuerprivilegvergabe des Staates zur Erzielung von gruppenspezifischen Verteilungsvorteilen in den Mittelpunkt der Reformdiskussion. Die Regierung wird im Rahmen ihres finanziellen Handlungsspielraumes solche (kleinen) Steuerreformen anstreben, die eine sukzessive Steuerentlastung jener Gruppen bewirken, von deren Unterstützung sie sich den Wahlsieg verspricht. Der Wunsch nach spezifischen (Verteilungs-) Ergebnissen stellt allerdings das Konzept eines rationalen Steuersystems auf den Kopf. Zielsysteme sollten vorgegeben und die Ergebnisse der Besteuerung unter genau spezifizierten Annahmen abgeleitet werden. Die Umkehrung des Rationalitätsprinzips ersetzt den normativen Anspruch der OT durch eine positive "wenn-dann"-Beziehung. Der entscheidende Nachteil dieses Reformkonzepts besteht darin, daß Steuervorteile, die aus egoistischen Motiven heraus angestrebt und aus politischer opportunität bewilligt werden, nur zufällig in den Rahmen einer normativ begründeten Steuerpolitik passen werden.

Der Prozeß erfährt weitere Dynamik, weil die alten Steuervorteile in der Regel kapitalisiert werden, weshalb die ehemals vorhandenen Einkommensvorteile im Laufe der Zeit verschwinden. Gegenuber der Situation ohne Steuervergünstigungen führt die Abschaffung von alten Steuerprivilegien zu Einkommensverlusten 
jener Individuen, die ihre Entscheidungen auf der Bas is bestehender Steuergesetze getroffen haben. Da immer neue Interessengruppen Steuervergünstigungen wünschen, während bestehende hartnäckig verteidigt werden, kommt eine Subventionsspirale in Gang, die nur durch eine sukzessive Erhöhung der Steuersätze in anderen Bereichen der Volkswirtschaft finanziert werden kann.

Dieses System immanenter Fehlentwicklungen des steuerpolitischen Willensbildungsprozesses legt eine Reform der fiskalischen Rahmenbedingungen nahe, die in Kapitel VIII untersucht wird. Statt der Korrektur einzelner Steuergesetze muß diesem steuerpolitischen Staatsversagen eine Steuerverfassung gegenübergestellt werden, die gemäß der Theorie des Zweitbesten die Verzerrungen des Mehrheitswahlsystems kompensiert und somit zur systematisierung demokratischer Steuerreformprozesse beiträgt. Die einstimmig verabschiedeten konstitutionellen Steuerregeln haben den Vorzug, daß sie effizient, gerecht und der individuellen Freiheit förderlich sind. Anders als die für den Leviathan konzipierten ergebnisbezogenen fiskalischen Restriktionen, die die Flexibilität der Steuerpolitik in der Regel sehr begrenzen, erscheinen verfahrensmäßige Steuerregeln, wie z.B. das Kquivalenzprinzip, das Gebot der horizontalen Gerechtigkeit oder das Gleichheitsprinzip von Hayek, besser geeignet, das Steuermonopol des Staates in kontrollierbaren Bahnen zu halten.

Neben den bekannteren Verfahrensregeln wird eine Steuerregel begründet, die einerseits eine verfassungsrechti iche Begrenzung von Steuerprivilegien vorsieht und andererseits einen konstitutionell beschlossenen Kriterienkatalog aufstellt, an den die Regierung bei der Vergabe von Steuervorteilen gebunden ist. Der Vorzug der Regel wird offenkundig, wenn man bedenkt, daß mit der Reduktion der Steuerprivilegien gleichzeitig auch die Einkommensteuersätze gesenkt werden können. Da sich die Allokationsverluste proportional zum Quadrat der Steuersätze ver- 
mindern, dürften mit einer derartigen Maßnahme ausreichende Allokationsgewinne verbunden sein, um die potentiellen Verlierer von Steuerprivilegien durch Senkung der Steuersätze kompensieren zu können. Die Arqumente belegen, daB mit Hilfe geeigneter Verfassungsregeln dauerhafte Wohlfahrtsgewinne erzielt werden können. Die wesentliche Aufgabe der Steuerpolitik besteht somit nicht in der Korrektur einzelner steuergesetze, sondern in der Konzeption einer detailierten Finanzverfassung, die sowoh? die Gefahr der Ausbeutung der Zensiten als auch der Unsystematik demokratischer Steuerreformen entgegenwirkt. 


\section{MATHEMATISCHER ANHANG}

\section{Anhang A: Zur Interpretation der Lagrange-Multiplikatoren}

Bevor man Gleichung (II.34) zu einer Interpretation von Steuerregeln nutzen kann, muß das Vorzeichen von $k$ bestimmt werden. Dazu nutzt man die Symmetrieeigenschaft der Substitutionseffekte

$$
\text { (A.1) } \quad s_{i k}=s_{k i} \text {, }
$$

multipliziert dann (II.34) mit $t_{k}$ und summiert schlieBlich über alle $k$, so da $B$ folgt:

$$
\text { (A.2) } \quad \sum_{i k} t_{i} s_{k i} t_{k}=\sum_{k} t_{k} x_{k}^{c}=k \bar{T}^{c} \text {. }
$$

Da die quadratische Form auf der linken Seite von (II.41) negativ definit ist, d.h.

$$
\text { (A.3) } \quad \sum_{i k} t_{i} s_{k i} t_{k}<0
$$

und das Steueraufkommen $\bar{T}^{C}$ positiv, muß $k$ negativ sein.Gleichung (II.35) kann schlieBlich mit der Steuerformel verglichen werden, die aus dem üblichen Optimierungsansatz mit Hilfe der indirekten Nutzenfunktion abgeleitet wird ${ }^{1)}$.

Aus dem alternativen Lagrange-Ansatz

$$
\text { (A.4) } L=v(q, m)+\alpha m+\sum_{i=2} t_{i} x_{i}(q, m)+\sum_{i=1} p_{i} y_{i}-p_{z} z
$$

folgt unter Berücksichtigung der Bedingungen erster Ordnung ${ }^{2}$ :

1) $\mathrm{Vgl}$. zur Ableitung und Interpretation optimaler Steuerregeln mit Hilfe der indirekten Nutzenfunktion z.B. Sandmo (1976). Atkinson/Stiglitz (1980), Kap. 12 und 14.

2) Vgl. Atkinson/Stiglitz (1980), S. 429 
(A.5) $\quad \sum_{i k} t_{i} s_{k i} t_{k}=\nu \bar{T}$

mit $v=-\frac{(\alpha-\lambda)}{\alpha}+t_{i} \frac{\partial x_{i}}{\partial m}$ und $\bar{T}=-\sum_{i=1}^{n} p_{i} y_{i}+p_{z} z$.

Da die rechte Seite von (A.5) unabhängig von $\nu$ ist, müssen beide Steuerregeln gleich interpretiert werden. Die wohlwollende Regierung wird daher dieselbe optimale Steuerstruktur wählen. Die Niveaus ihrer Kontrollvariablen, d.h. der Steuersätze bzw. Konsumentenpreise (mit $m=0$ ) werden sich daher im optimum entsprechen. Bei vorgegebenen Marktpreisen und Einkommen wird der Konsument sowohl im primalen als auch im dualen Optimierungsansatz denselben Konsumgütervektor realisieren. Dies impliziert,daß die Regierung in jedem Fall ihr Steueraufkommen $\overline{\mathrm{T}}^{\mathrm{C}}=\overline{\mathrm{T}}$ realisiert und ihre Budgetrestriktion im optimum jeweils gleiche Werte aufweist, so daß $\alpha=\theta$ gilt.

Setzt man die rechte Seite von (A.2) und (A.5) gleich:

(A.6) $\quad-\frac{\theta-\mu}{\theta}=-\frac{\theta-\lambda}{\theta}+\sum_{i} t_{i} \frac{\partial x_{i}}{\partial m}$,

so folgt:

(A.7) $\mu=\lambda+\underset{i}{\theta \Sigma_{i}} t_{i} \frac{\partial x_{i}}{\partial m}$.

Der Lagrange-Multiplikator $\mu$ definiert den sozialen Grenznutzen des Einkommens für den Konsumenten; dieser besteht einerseits aus seinem Grenznutzen des Einkommens $(\lambda)$ und andererseits aus dem gesellschaftlichen Wert der zusätzlichen Steuern, die über den Einkommenseffekt erzielt werden können ${ }^{1)}$ :

1) Vgl. Diamond (1975), S. 338 oder Munk (1978), S. 11 
$\sum_{i} t_{i} \frac{\partial x_{i}}{\partial m} \quad$ gewichtet mit $\theta$.

Unter der Voraussetzung, daB die Regierung eine optimale Menge des öffentlichen Gutes $\bar{z}$ anbietet und die demprivaten Sektor entzogenen Ressourcen $\sum p_{i} y_{i}^{g}$ optimal einsetzt, gilt:

(A.8)

$$
\frac{\partial L}{\partial\left(\sum_{i} p_{i} y_{i}^{g}+p_{z} \bar{z}\right)}=\frac{\partial u}{\partial T}=\theta
$$

weil jede zusätzliche Steuererhebung eine Mehrbelastung verursacht, die über die NutzeneinbuBen hinausgeht, die zur Finanzierung des optimalen Ausgabenniveaus notwendig sind. $\theta$ kann als Grenznutzen des zusätzlichen Steueraufkommens interpretiert werden. Im Optimum muß von $\theta<0$ ausgegangen werden. Da $k$ negativ ist, gilt $-(\theta-\mu)>0$; bzw. wegen $(I I .27)$ und des Theorems für Lagrange-Multiplikatoren ${ }^{1)} \partial L / \partial m=\partial u / \partial m$ :

$$
\text { (A.9) } \frac{\partial L}{\partial m}=\frac{\partial u}{\partial m}=\mu-\theta>0 \text {. }
$$

Somit gilt:

OT Regel $A^{2)}$ :

Wenn das geplante Steueraufkommen $\bar{T}$ nicht völlig durch Pauschalsteuern gedeckt wird und be $i$ jedem Pauschalsteueraufkommensniveau eine optimale Second-best-Besteuerungerfolgt, dann steigert jede kleine Erhöhung der Pauschalsteuern die Wohlfahrt des repräsentativen Konsumenten.

1) Siehe Z.B. Green (1975), S. 100

2) Dixit/Munk (1977), S. 106 leiten ein entsprechendes Ergebnis unter Berücksichtigung positiver Unternehmergewinne $a b$. Dasselbe Ergebnis resultiert, wenn man Pauschalgewinneinkommen der Haushalte besteuert. 
Anhang B: Definition und Messung der Mehrbelastung ${ }^{1}$ )

Anhand von Abb. B sollen sowohl die Definition als auch die MeBkonzeption der Mehrbelastung demonstriert werden. Es wird beispielhaft nur das Konzept der equivalent variation (EV) ${ }^{2}$ ) betrachtet. Die EV definiert die Mehrbelastung als den maximalen Betrag, den der Besteuerte über das Gütersteueraufkommen hinaus zu zahlen bereit wäre, wenn statt der Steuer auf $x$ eine Pauschalsteuer gleicher Höhe abzuführen wäre. Abb. B zeigt nun c.p. die Einführung einer Steuer $t$ auf Gut $x$, weshalb sich die urspruing1 iche Budgetgerade $M_{0} L$ nach $M_{0} K$ dreht. Der daraus resultierende gesamte Wohlfahrtsverlust für den Konsumenten beträgt:

$$
\begin{aligned}
M_{0} M^{\prime}= & M_{0} R+R T-M^{\prime} T=q^{1} x_{2}+x_{2} B C x_{3}-x_{3} q^{1}= \\
& O q^{1} B x_{2}+x_{2} B C x_{3}-O q^{0} C x_{3}=q^{1} B C q^{0}
\end{aligned}
$$

bzw.

$$
\text { (B.2) } \quad M_{0} M^{\prime}=e\left(q^{0}, u_{0}\right)-e\left(q^{0}, u_{1}\right)=e\left(q^{1}, u_{1}\right)-e\left(q^{0}, u_{1}\right) \text {. }
$$

Da die EV das neue Nutzenniveau $u_{1}$ als Referenzsituation aufweist, wird vom gesamten Wohlfahrtsverlust die Steuerzahlung $M_{0} M^{\prime \prime}$ in Punkt II subtrahiert. Die Mehrbelastung entspricht daher:

$$
\text { (B.3) } \quad \Delta W_{E V}=M_{0} M^{1}-t x_{2}=q^{1} B C q^{0}-q^{1} B D q^{0}=B C D
$$

bzw.

$$
\Delta W_{E V}=M_{0} M^{\prime}-t x_{2}=e\left(q^{1}, u_{1}\right)-e\left(q^{0}, u_{1}\right)-t x^{*}\left(q^{1}, u_{1}\right),
$$

1) Die Analyse der Nutzenmessung geht auf Dupuit (1969), Marshall (1961) und Hotelling (1938) zurück. Die Definition des EV-Konzeptes und weitere Maße stammt von Hicks (1943). Vgl. zur neueren Diskussion von Mehrbelastungen Harberger (1979), Green/Sheskinski (1979). Silberberg (1972), Hausman (1981). Zur Messung der Mehrbelastung mit Hilfe der Ausgabenfunktion siehe Diamond/McFadden (1974), Kay (1980) und zur graphischen Darstellung alternativer Meßkonzepte Mann (1982)

2) $\mathrm{Vgl}$. dazu auch Hicks (1943), S. $31 \mathrm{ff}$. und zu einem Oberblick über we itere Mehrbelastungsmaße z.B. Silberberg (1972) 


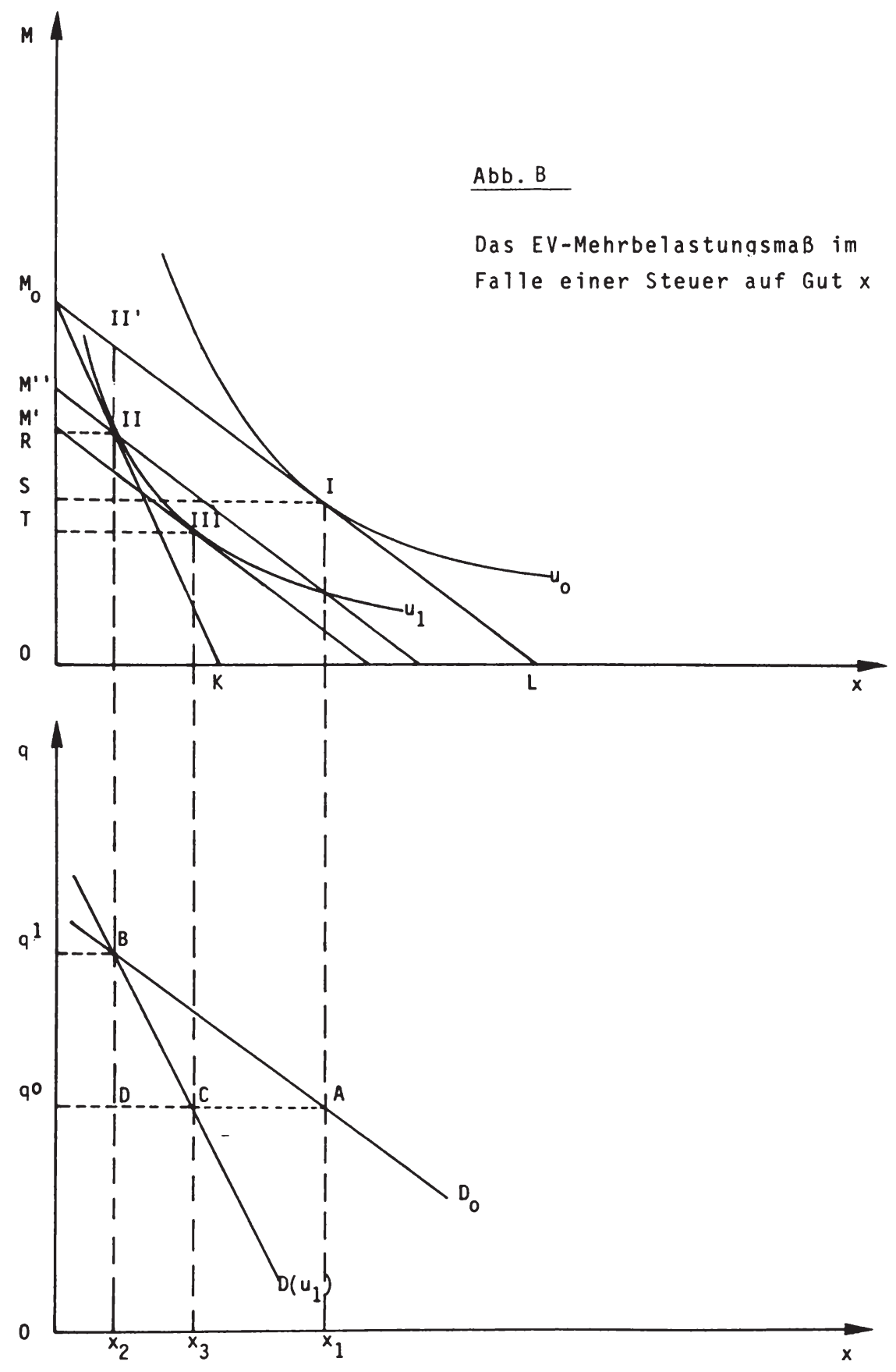

Hugo Mann - 978-3-631-75532-7

Downloaded from PubFactory at 01/11/2019 03:48:02AM

via free access 
d.h. die Mehrbelastung entspricht genau dem Dreieck BCD unterhalb der kompensierten Nachfragefunktion $D\left(u_{q}\right)$. Daher kannman imFalle einer linearen kompensierten Nachfragefunktion auf dem Markt für Gut $x$ als Maß für die Mehrbelastung näherungsweise auch schreiben:

(B. 5$) \quad \Delta W=\frac{1}{2} \Delta q \Delta x=\frac{1}{2} t^{2} \eta q x$,

wobei $\Delta q$ die Marktpreisänderung und $\Delta x$ die Mengenänderung von $x$ darstellen. Der Steuersatz $t$ entspricht $\Delta q_{x} / q$ und $n$ bezeichnet die Nachfrageelastizität entlang der Nachfragekurve mit konstantem Aufkammen ${ }^{1)}$. Gleichung (B.5) belegt, daB der Wohlfahrtsverlust im Quadrat zum Steuersatz ansteigt.

In $A b b$. B wird ferner deutlich, daB aufgrund der Subtraktion des Steueraufkommens $t x_{2}$ der Mehrbelastungseffekt M'M" ausschlieBlich durch den Substitutionseffekt entlang der Indifferenzkurve $u_{1}$ erzeugt wird, d.h. die Einkommenseffekte, die in der normalen Nachfragefunktion berücksichtigt werden, entfallen.

Das als $\triangle W_{E V}$ definierte EV-Mehrbelastungskonzept hat den Vorteil, daB die Minimierung der Mehrbelastung, d.h. Minimierung unwiderbringlicher Nutzenverluste, unter der Nebenbedingung eines gegebenen steueraufkommens

min.: $\quad e\left(q^{1}, u_{1}\right)-e\left(q^{0}, u_{1}\right)-t x *\left(q^{1}, u_{1}\right)$

u.d.N.: $\quad T=t x_{2}=t x *\left(q^{1}, u_{1}\right)$

zu denselben Ergebnissen führt, wie der Optimierungsansatz $(\text { II. 27 })^{2)}$.

1) Vgl. Boadway/Wildasin (1984), S. 388

2) Vgl. Kay (1980) oder Mann (1982) oder Rose/Wiegard (1983), S. 27 ff. 
Anhang C: Pauschalsteuern als Kennzeichen der direkten Besteuerung

Die Behauptung Pauschalsteuern seien ein Kennzeichen der direkten Besteuerung kann dadurch veranschaulicht werden, daB man die Ausgabenfunktion (II.12) wie folgt schreibt:

(c.1) $\sum_{i=2}^{n}\left(p_{i}+t_{i}\right) x_{i}^{c}=x_{1}^{c}\left(1-t_{1}\right)+m$,

wobe $i x_{1}^{c}$ für das kompensierte Arbeitsangebot und $p_{1}=1$ für den Lohnsatz stehen.

Dividiert man die Summanden in Gleichung (C.1) durch $\left(1-t_{q}\right)$, erhält man bei konstanten Produzentenpreisen höhere Konsumentenpreise bzw. Steuerbeträge pro Mengeneinheit

$$
\text { (c.2) } \frac{p_{i}+t_{i}}{1-t_{1}}=\frac{q_{i}}{1-t_{1}} \text {, }
$$

die den Steuerausfall aus nicht-versteuertem Lohneinkommen kompensieren. Setzt man

(c.3) $\frac{p_{i}+t_{i}}{1-t_{1}}=p_{i}+t_{i}^{\prime}$, mit $t_{i}^{\prime}=\frac{t_{i}+p_{i} t_{1}}{1-t_{1}}$,

so ändert sich die Ausgabenfunktion zu:

(c.4) $\sum_{i=2}^{n}\left(p_{i}+t_{i}^{\prime}\right) x_{i}^{c}=x_{1}^{c} p_{1}+\frac{m}{1-t_{1}}=x_{1}^{c} p_{1}+m^{\prime}$,

so daB $\mathrm{m}^{\prime}$ Ausdruck individueller Besteuerung ist. 
Anhang D: Zur Ableitung der "Optimierungsergebnisse" (IV.14) mit Hilfe des dualen Maximierungsansatzes

Statt (II.27) resultiert dann der Optimierungsansatz

$$
\begin{aligned}
& \text { (D.1) } \quad \max : T=\sum_{i=2}^{n} t_{i} x_{i}(q, u)+\sum_{i=1}^{n} p_{i} y_{i}^{g}-m-p_{z} z \\
& \text { u.d.N.: } m-e(q, u)=0
\end{aligned}
$$

bzw.

(D.2) $L=\sum_{i} t_{i} x_{i}(q, u)+\sum_{i} p_{i} y_{i}^{g}-m-p_{z} z+\psi[m-e(q, u)]$

Die Bedingungen erster Ordnung für ein Maximum lauten ${ }^{1)}$ :

(D.3) $\quad \frac{\partial L}{\partial q_{k}}=\sum_{i} t_{i} \frac{\partial x_{i}^{c}}{\partial q_{k}}+x_{k}^{c}-\psi \frac{\partial e(q, u)}{\partial q_{k}}=0$, mit $k=2, \ldots, n$

(D.4) $\quad \frac{\partial L}{\partial m}=-1+\psi=0$

Unter Berücksichtigung von $\partial x_{i}^{c} / \partial q_{k}=s_{i k}, \partial e(q, u) / \partial q_{k}=x_{k}^{c}$ und nach Einsetzen von (D.4) in (D.3) folgt unmittelbar:

(D.5) $\quad \sum_{i} t_{i} s_{i k}=0$

1) Die Bedingungen zweiter Ordnung werden als erfüllt unterstellt. Außerdem wird auf die hier irrelevanten partiellen Ableitungen $\partial \mathrm{L} / \partial \mathrm{u}$ und $\partial \mathrm{L} / \partial \psi$ verzichtet. $\mathrm{Vgl}$. dazu Gleichung (II.27). 
Anhang E: Die Berechnung eines Kriteriums zur schrittweisen Erhöhung des Steueraufkommens

Aus (VII.4) folgt unter Berücksichtigung der Roy'schen Identität (E.1) $\quad d v=\sum_{i=1}^{n} \frac{\partial v}{\partial m} x_{i} d q_{i}+\frac{\partial v}{\partial m} d m=0$ mit $i=1, \ldots, n$.

Nach Division von (E. 1) durch $\partial v / \partial m$ und unter Berücksichtigung von $d q_{i}=d t_{i}$ folgt:

(E.2) $\quad \frac{d v}{\partial v / \partial m}=-\sum_{i} x_{i} d q_{i}+d m=0$.

Einsetzen der slutsky-Gleichung $\partial x_{i} / \partial q_{k}=s_{i k}-x_{k}\left(\partial x_{i} / \partial m\right)$ und (E.2) in (VI.3) ergibt:

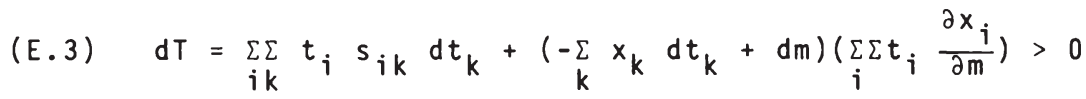
$i, k=2, \ldots, n$

und unter Berücksichtigung von (E.2) in (E.3) folgt:

(E.4) $\quad d T=\sum_{i k} t_{i} s_{i k} d t_{k}>0$. 
Anhang F: Zur Ableitung von Gleichung (VII.20)

Differenziert man (VII.15) und (VII.16) nach $q_{j}$

(F.1) $\quad \frac{\left.\partial \mu_{(j} T_{i}\right)}{\partial q_{i}}=\frac{\partial\left[\sum_{i} \mu_{T}\right]}{\partial q_{i}}=\frac{\partial \mu_{T}}{\partial q_{i}}$

(F.2) $\frac{\partial \sigma_{\left(\sum T_{i}\right)}}{\partial q_{i}}=\frac{\sigma_{T_{i}}}{\underbrace{}_{i}\left(\sum_{i}\right)} \frac{\partial \sigma_{T_{i}}}{\partial q_{i}}+\frac{\partial \operatorname{cov} v_{T}}{\partial q_{i}}$

und setzt (F.1) und (F.2) in (VII.18) e in

(F.3) $\quad \frac{\partial V}{\partial q_{i}}=\frac{\partial V}{\partial \mu_{T_{i}}} \frac{\partial \mu_{T_{i}}}{\partial q_{i}}+\frac{\partial V}{\left.\partial \sigma_{(i} T_{i}\right)} \cdot\left[\frac{1}{\partial \sigma_{\left(\sum_{i} T_{i}\right)}}\left(\sigma_{T_{i}} \frac{\partial \sigma_{T_{i}}}{\partial q_{i}}\right)+\frac{\partial \operatorname{cov} V_{T}}{\partial q_{i}}\right]=0$

und berücksichtigt in (F.3) das RisikomaB

$(F .4) \quad R=R(q)=-\frac{\partial V / \partial \sigma\left(\Sigma_{i} T_{i}\right)}{\partial V / \partial \mu\left(\Sigma_{i} T_{i}\right)}=-\frac{\frac{\partial V}{\partial \mu}}{\frac{\partial V}{\partial \sigma}}$

mit $\mu^{*}=\mu_{\left(\sum_{i} T_{i}\right)}$ und $\sigma^{*}=\sigma_{\left(\sum_{i} T_{i}\right)}$, so folgt:

(F.5) $\quad \frac{\partial \mu_{T}}{\partial q_{i}}-R\left[\frac{1}{\sigma\left(\sum_{i} T_{i}\right)}\left(\sigma_{T_{i}} \frac{\partial \sigma_{T_{i}}}{\partial q_{i}}\right)+\frac{\partial c o v_{T}}{\partial q_{i}}\right]=0$.

Differenziert man (VII.12) und (VII.13) nach $q_{j}$ 
(F.6) $\quad \frac{\partial \mu_{T_{i}}}{\partial q_{i}}=\frac{\partial \mu_{T_{i}}}{\partial t_{i}}=\mu_{x_{i}}+t_{i} \frac{\partial \mu_{x_{i}}}{\partial t_{i}}$

(F.7) $\quad \frac{\partial \sigma_{T_{i}}}{\partial q_{i}}=\sigma_{x_{i}}+t_{i} \frac{\partial \sigma_{x_{i}}}{\partial t_{i}}$

und setzt $(F .6)$ sowie $(F .7)$ in $(F .5)$ ein

(F.8) $\quad t_{i}-\frac{\partial \mu_{x_{i}}}{\partial q_{i}}=-\mu_{x_{i}}+R\left[\frac{1}{\sigma\left(\sum_{i} T_{i}\right)}\left(\sigma_{x_{i}} t_{i}\left(\sigma_{x_{i}}+t_{i} \frac{\partial \sigma_{x_{i}}}{\partial q_{i}}\right)+\frac{\partial \operatorname{cov} v_{T}}{\partial q_{i}}\right)\right]$,

erhält man durch Auflösen nach den optimalen Steuersätzen $t_{j}$ :

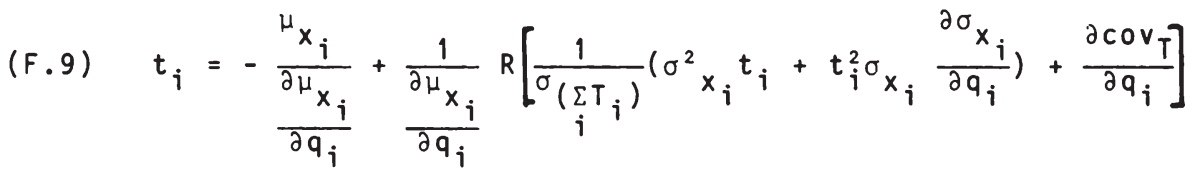

Multipliziert man Gleichung (F.g) mit $\frac{1}{q_{i}}$, so folgt:

$$
\begin{aligned}
(F .10) \quad \tau_{i}=\frac{t_{i}}{q_{i}}= & -\frac{1}{\varepsilon\left(\mu_{\left.x_{i}, q_{i}\right)}\right.}+\frac{1}{\varepsilon\left(\mu_{\left.x_{i}, q_{i}\right)}\right)} \frac{1}{\mu_{x_{i}}} \\
& {\left[\frac{1}{\sigma\left(\sum_{i} T_{i}\right)}\left(\sigma^{2} x_{i} t_{i}+t_{i}^{2} \sigma_{x_{i}} \frac{\partial \sigma_{x_{i}}}{\partial q_{i}}\right)+\frac{\partial \operatorname{cov} v_{T}}{\partial q_{i}}\right] . }
\end{aligned}
$$




\section{LITERATUR}

Abbott, M., Ashenfelter, 0. (1976) Labour supply, commodity demand and the allocation of time, in: Review of Economic Studies 43 , S. $389-412$

Abgabenordnung (1977), in: Beck'sche Textausgaben, München 1977

Agrarberichte (1971 - 1984) Bundesministerium für Ernährung, Landwirtschaft und Forsten (Hrsg.)

Albers, W. (1977) Ziele und Bestimmungsgründe der Finanzpolitik, in: Andel, N., Haller, H., Neumark, F. (Hrsg.), Handbuch

der Finanzwissenschaft, Bd. I, Tübingen, S. $123-163$

Allingham, M.G., Sandmo, A. (1972) Income tax evasion: A theoretical analysis, in: Journal of Public Economics 1 , S. $323-338$

Almsick v., J. (1981) Die negative Einkommensteuer, Berlin

Alt, J.E. (1983) The evolution of tax structures, in: Public Choice 41, S. $181-222$

Andel, N. (1977) Subventionen, in: Albers, W. u.a. (Hrsg.), Handwörterbuch der Wirtschaftswissenschaft, Bd. 7, Stuttgart u.a.0., S. $491-510$

Arrow, K.J. (1963) Social Choice and Individual Values, 1 st ed. New York 1951, 2nd ed. New York 1963

Atkinson, A.B. (1973) How Progressive Should Income Tax Be?, in: Parkin, M. (Ed.), Essays in Modern Economics, London, S. $90-109$

Atkinson, A.B. (1977) Optimal Taxation and the direct versus indirect tax controversy, in: Canadian Journal of Economics 10 , S. $590-606$

Atkinson, A.B., Stiglitz, J.E. (1972) The Structure of Indirect Taxation and Economic Efficiency, in: Journal of Public Economics 1, S. $97-119$

Atkinson, A.B., Stiglitz, J.E. (1976) The Design of Tax Structure: Direct versus Indirect Taxation, in: Journal of Public Economics 6, S. $55-76$

Atkinson, A.B., Stiglitz, J.E. (1980) Lectures on Public Economics, Maidenhead, Berkshire

Bails, D. (1982) A critique on the effectiveness of taxexpenditure limitations, in: Public Choice 38, S. $129-138$ 
Baldry, J.C. (1979) Tax evasion and labour supply, in: Economic Letters 3, S. $53-56$

Bamberg, G., Baur, F. (1980) Statistik, München, Wien

Barro, R.J. (1984) Macroeconomics, New York u.a.0.

Bartlett, R. (1973) Economic Foundations of Political Power, New York, London

Baum, Th.M. (1982) Staatsverschuldung und Stabilisierungspolitik in der Demokratie, Frankfurt a.M., Bern

Baumol, W.J. (1967) Macroeconomics of Unbalanced Growth: The Anatomy of Urban Crisis, in: American Economic Review 57 , S. $415-426$

Becker, G.S. (1983) A theory of competition among pressure groups for political influence, in: The ouarterly Journal of Economics 99, S. $371-400$

Benda, E., Kreuzer, K. (1973) Verfassungsrechtliche Grenzen der Besteuerung, in: Deutsche Steuer-Zeitung 61 , S. $49-57$

Benkert, W. (1983) Die Steuerrevolten in den USA - Ablauf, Ursachen und Auswirkungen, in: Wirtschaftsstudium 10, S. $471-476$

Ben-Porath, Y., Bruno, M. (1977) The Political Economy of a Tax Reform, in: Journal of Public Economics 7, S. $285-307$

Bentham, J. (1907) An Introduction to the Principles of Morals and Legislation, Oxford

Bergson, A. (1938) A reformulation of certain aspects of welfare economics, in: The Quarterly Journal of Economics 52, S. $314-344$

Bernholz, P. (1972) Grundlagen der Politischen ökonomie, 1. Bd., Tübingen

Bernholz, P. (1975) Grundlagen der Politischen ökonomie, 2. Bd., Tübingen

Bernholz, P. (1981) Die Zunahme der Staatstätigkeit: Ursachen und Konsequenzen für den freiheitlichen Rechtsstaat, in: IHS-Journal 5, S. $87-108$

Bernholz, P., Breyer, F. (1984) Grundlagen der Politischen ökonomie, 2. Aufl., Tübingen

Bittker, B. (1975) Tax Shelters and Tax Capitalization or Does the Early Bird Get a Free Lunch, in: National Tax Journal 28 , S. $416-419$ 
Bitz, M. (1981) Entscheidungstheorie, München

Blackorby, Ch., Diewert, W.E. (1979) Expenditure Functions, Local Duality, and Second Order Approximations, in: Econometrica 47 , S. $579-601$

Blankart, Ch.B. (1977) Neuere Ansätze zur Erklärung des Wachstums der Statstätigkeit - Ein interpretierender überblick, in: Hamburger Jahrbuch für Wirtschafts- und Gesellschaftspolitik, Tübingen, $S .73 \mathrm{ff}$.

Blankart, Ch.B. Bongaerts, J.C. (1981) Institutional Aspects of Optimal Taxation, Diskussionspapier Juni 1981

Boadway, R.W. (1975) Optimal Taxes with Untaxed Goods and Factors, in: Public Finance Quarterly 3, S. $275-290$

Boadway, R.W. (1979) Public Sector Economics, Cambridge, Mass.

Boadway, R.W., Bruce, N. (1984) Welfare Economics, Oxford

Boadway, R.W., Wildasin, D.E. (1984) Public Sector Economics, 2nd ed., Boston, Toronto

Bös, D., Tillmann, G. (1983) Neid und progressive Besteuerung, in: Hansmeyer, K.-H. (Hrsg.), Staatsfinanzierung im Wandel, Berlin, S. $637-660$

Borcherding, T.E. (1977) One Hundred Years of Public Spending, 1870 - 1970, in: Borcherding, T.E. (Ed.), Budgets and Bureaucrats: The Sources of Government Growth, Durham, S. $3-18$

Borcherding, T.E. (1981) The Growth of US. Public Spending: Another Look, Working Paper prepared for the Conference of Constraining Federal Taxing and Spending, The Hoover Institution Stanford University, October $21-23,1981$

Boskin, M.J. (1978) Federal Tax Reform: Myths and Realities, San Francisco

Bradford, D.F., Rosen, H.S. (1976) New Developments in Public Finance, The Optimal Taxation of Commodities and Income, in: American Economic Review 66, S. 94 - 101

Break, G., Pechman, J. (1975) Federal Tax Reform The Impossible Dream?, Washington D.C.

Brennan, G. (1981) Tax Limits and The Logic of Constitutional Restrictions, in: Ladd, H.F., Tidemann, N.T. (Eds.), Tax and Expenditure Limitations, Washington D.C., S. $121-138$ 
Brennan, G., Buchanan, J.M. (1977) Towards a Tax Constitution for Leviathan, in: Journal of Public Economics 8 , S. $255-273$

Brennan, G., Buchanan, J.M. (1978) Tax Instruments as Constraints on the Disposition of Public Revenues, in: Journal of Public Economics 9, S. $301-318$

Brennan, G., Buchanan, J.M. (1979) The Logic of Tax Limits: Alternative Constitutional Constraints on the Power to Tax, in: National Tax Journal $32, \mathrm{~S} .11-22$

Brennan, G., Buchanan, J.M. (1980) The Power to Tax, Cambridge u.a.0.

Brennan, G., McGuire, T. (1975) Optimal Policy Choice under Uncertainty, in: Journal of Public Economics 4, S. $205-209$

Breton, A. (1978) Economics of Representative Democracy, in: The Institute of Economics Affairs (Ed.), The Economics of Politics, Lancing, S. $51-64$

Breuel, B. (1983) Eine Wirtschaft fürs Museum, in: Die Zeit, $\mathrm{Nr} .43,21$. Oktober, S. $36 \mathrm{f}$.

Broome, J. (1975) An important theorem on income tax, in: Review of Economic Studies 42, S. $649-652$

Brown, J., Deaton, A. (1972) Surveys in applied economics: models of consumer behaviour, in: Economic Journal 82 , S. $1145-1236$

Browning, J. (1979) Estimating the Welfare Cost of Tax Preferences, in: Public Finance Quarterly 7, S. $199-219$

Brunner, J.K. (1984) Zur Funktion mathematischer Modelle in der Nationalökonomie, in: Jahrbuch für Nationalökonomie und Statistik 199, S. $308-320$

Buchanan, J.M. (1963) The Economics of Earmarked Taxes, in: Journal of Political Economy 71, S. $457-469$

Buchanan, J.M. (1967) Public Finance in Democratic Process, Chapel Hill

Buchanan, J.M. (1975) The Limits of Liberty, Between Anarchy and Leviathan, Chicago, London

Buchanan, J.M. (1976a) Taxation in Fiscal Exchange, in: Journal of Public Economics $6,5.17-29$

Buchanan, J.M. (1976b) A Hobbesian interpretation of the Rawls difference principle, in: Kyklos 29, S. $5-25$ 
Buchanan, J.M. (1977a) Freedom in Constitutional Contract, Texas

Buchanan, J.M. (1977b) Why does Government grow?, in: Borcherding, T.E. (Ed.), Budgets and Bureaucrats: The Sources of Government Growth, Durham, S. 3 - 18

Buchanan, J.M. (1978) From Private Preferences to Public Philosophy: The Development of Public Choice, in: The Institute of Economic Affairs (Ed.), Lancing, S. $1-20$

Buchanan, J.M. (1979) Constitutional Constraints on Governmental Taxing Power, in: Ordo, S. $349-359$

Buchanan, J.M. (1980a) Procedural and Quantitative Constitutional Constraints of Fiscal Authority, in: Moore, W.S., Penner, R.G. (Eds.), The Constitution and the Budget, Washington D.C.

Buchanan, J.M. (1980b) Rent Seeking and Profit Seeking, in: Buchanan, J.M., Tollison, R.B., Tullock, G. (Eds.), Towards a Theory of the Rent-Seeking-Society, Texas, S. $3-15$

Buchanan, J.M. (1983) Rights, Efficiency, and Exchange: The Irrelevance of Transactions Cost, Paper prepared for presentation at Verein für Socialpolitik, Conference on Property Rights, Basel

Buchanan, J.M., Brennan, G. (1980) Tax Reform without Tears, in: Aaron, H.J., Boskin, M.J. (Eds.), The Economics of Taxation, Washington D.C., S. $33-53$

Buchanan, J.M., Brennan, G. (1981) Tax Specification and Tax Limitation, Manuscript, Conference on Constraining Federal Taxing and Spending, Hoover Institution, Stanford

Buchanan, J.M., Flowers, M.R. (1980) The Public Finances. An Introductory Textbook, 5 th ed, Homewood, 111 .

Buchanan, J.M., Tollison, R.D., Tullock, G. (Eds.) (1980) Towards a Theory of the Rent-Seeking Society, Texas

Buchanan, J.M., Tullock, G. (1962) The calculus of consent, 5 th ed. 1974, Ann Arbor

Buchanan, J.M., Wagner, R.E. (1977) Democracy in Deficit. The Political Legacy of Lord Keynes, New York u.a.0.

Bund, D. (1984) Die okonomische Theorie der Verfassung, BadenBaden

Bund der Steuerzahler, Präsidium (Hrsg.) (1983) Grenzen für den Steuerstaat, Referate und Protokolle vom Deutschen Steuerzahlerkongreß 1983 in Düsseldorf, Bonn 
Bundesministerium der Finanzen (Hrsg.) (1971) Gutachten der Steuerreformkomm ission, Bonn

Bundesministerium der Finanzen (1984) Leitlinien zur Neuregelung der steuerlichen Förderung des selbstgeschützten Wohneigentums , 25

Bundesministerium der Finanzen (1984) Finanznachrichten des Bundesministeriums der Finanzen 14, S. 2

Bundesregierung (1983) Bulletin des Presse- und Informationsam tes der Bundesregierung 133, S. 1211

Bundesregierung (1983) Bulletin des Presse- und Informationsamtes der Bundesregierung 85 , S. 782 und 86, S. 798

Caesar, R. (1980) Steuerquoten, Steuerstrukturen und Steuerharmonisierung in der Europäischen Gemeinschaft, Finanzwissenschaftliches Forschungsinstitut an der Universität Köln, Sonderveröffentli chung Nr. 3, Köln

Christiansen, V. (1980) Two Comments on Tax Evasion, in: Journal of Public Economics 13, S. $389-393$

Church, A.M. (1974) Capitalization of the Effective Property Tax Rate on Single Family Residences, in: National Tax Journal 27, S. $133-142$

Citrin, J. (1978) The Alienated Voter, in: Taxing and Spending 1

Corlett, W.J., Hague, D.C. $(1953 / 54)$ Complementarity and the Excess Burden of Taxation, in: Review of Economic Studies 21, S. $21-30$

Courant, P., Rubinfeld, D. (1981) On the welfare effects of tax limitations, in: Journal of Public Economics 16, S. $289-316$

Darrough, M.N., Southey, C. (1977) Duality in consumer theory made simple: The revealing of Roy's identity, in: Canadian Journal of Economics 10, S. $307-317$

Dasgupta, P., Stiglitz, J. (1972) On Optimal Taxation and Public Production, in: Review of Economic Studies 39, S. $87-103$

Davis, 0.A., Dempster, M.A., Wildavsky, A. (1979) Zu einer prädikativen Theorie der Regierungsausgaben. Die inländischen Budgetanweisungen in den USA, in: Pommerehne, W.W., Frey, B.S. (Hrsg.), Ökonomische Theorie der Politik, Berlin, Heidelberg, New York, S. $369-377$

Deaton, A.S. (1975) The measurement of income and price elasticities, in: European Economic Review 6, S. $261-274$ 
Deaton, A.S. (1977) Equity, efficiency and the structure of indirect taxation, in: Journal of Public Economics 8, S. $299-312$

Deaton, A.S. (1978) Specification and testing in applied demand analysis, in: Economic Journal 88, S. $524-536$

Deaton, A.S. (1979) The distance function in consumer behavior with application to index numbers and optimal taxation, in: The Review of Economic Studies 46, S. $391-405$

Deaton, A.S. (1981) optimal Taxes and the Structure of Preferences, in: Econometrica 49, S. $1245-1260$

Deaton, A.S., Muellbauer, J. (1980) Economics and consumer behavior, Cambridge

Diamond, P.A. (1975) A many-person Ramsey tax rule, in: Journal of Public Economics 4, S. $335-342$

Di amond, P.S., Helms, L.J., Mirrlees, J.A. (1980) Optimal Taxation in a Stochastic Economy. A Cobb-Douglas Example, in: Journal of Public Economics 14, S. $1-29$

Di amond, P.A., McFadden, D.L. (1974) Some Uses of the Expenditure Function in Public Finance, in: Journal of Public Economics 3, S. 3- 21

Diamond, P.A., Mirrlees, J.A. (1971a) Optimal Taxation and Public Production. Production Efficiency, in: American Economic Review 61 , S. $8-27$

Diamond, P.A., Mirrlees, J.A. (1971b) Optimal Taxation and Public Production. Tax Rules, in: American Economic Review 61, S. $261-278$

Diewert, $W$. (1978) Optimal tax perturbations, in: Journal of Public Economics 10, S. $139-177$

Diewert, W. (1982) Duality Approaches to Microeconomic Theory, in: Arrow, K.J., Intriligator, M.D., in: Handbook of Mathematical Economics, Vol. II, Amsterdam, New York, oxford, S. $535-599$

Dixit, A.K. (1970) On the Optimum Structure of Commodity Taxes, in: The American Economic Review 6, S. 295 - 301

Dixit, A.K. (1975) Welfare Effects of Tax and Price Changes, in: Journal of Public Economics 4, S. $103-123$

Dixit, A.K. (1979) Price Changes and Optimum Taxation in a Many-Consumer Economy, in: Journal of Public Economics 11 , S. $143-157$ 
Dixit, A.K., Munk, K.J. (1977) Welfare effects of tax and price changes: a correction, in: Journal of Public Economics 8 , S. $103-107$

Downs, A. (1957) An Economic Theory of Democracy, New York

Downs, A. (1960) Why the government budget is too small in a democracy, in: World Politics 13, S. $541-563$

Dreißig, W. (1984) Zur Konsolidierung der öffentlichen Haushalte, in: Finanzarchiv NF 42, S. $167-174$

Due, J. (1977) Personal Deductions, in: Pechman, J. (Ed.), Comprehensive Income Taxation, Washington D.C.

Dupuit, J. (1969) On the Measurement of the Utility of Public Works, in: Arrow, K., Scitovsky, T. (Eds.), Readings in Welfare Economics, Homewood, Ill., S. $255-283$

Eaton, J., Rosen, H.S. (1980a) Optimal Redistributive Taxation and Uncertainty, in: Quarterly Journal of Economics 95 , S. $357-364$

Eaton, J., Rosen, H.S. (1980b) Labor supply, uncertainty, and efficient taxation, in: Journal of Public Economics 14, S. $365-374$

Edel, M., Sclar, E. (1974) Taxes, spending and property values: supply adjustments in a Tiebout-0ates model, in: Journal of Political Economy 82 , S. 941 - 954

Felderer, B. (1979) Inflation, Wagnersches Gesetz und Stagnation, in: Finanzarchiv 37 , S. $223-269$

Feldstein, M.S. (1972) Distributional Equity and the Optimal Structure of Public Prices, in: American Economic Review 52 , S. $32-36$

Feldstein, M.S. (1973) On the Optimal Progressivity of the Income Tax, in: Journal of Political Economy 2, S. $357-376$

Feldstein, M.S. (1976a) On the theory of tax reform, in: Journal of Public Economics 6, S. $77-104$

Feldstein, M.S. (1976b) Compensation in Tax Reform, in: National Tax Journal 29, S. $123-130$

Feldstein, M.S. (1980) A Contribution to the Theory of Tax Expenditures: The Case of Charitable Giving, in: Aaron, H.J., Boskin, M.J. (Eds.), The Economics of Taxation, Washington D.C., S. $99-122$

Finanzberichte (1985) Bundesministerium der Finanzen (Hrsg.), Bonn, 1983 und 1985 
Finanznachrichten (1984) Bundesministerium der Finanzen (Hrsg.), $14 / 85$ vom 29.03.1984, Referat Presse und Information

Folkers, C. (1978) Der Reformakzelerator. Bemerkungen zu einer Reform des Einkommensteuertarifs, in: Wirtschaftsdienst 7 , S. $340-345$

Folkers, C. (1979) Staatswirtschaftliche Quoten und Beziehungszahlen, in: Das Wirtschaftsstudium 8, S. $405-409$ und 9 , S. $457-463$

Folkers, C. (1983a) Begrenzungen von Steuern und Staatsausgaben in den USA, Baden-Baden

Folkers, C. (1983b) Zu einer positiven Theorie der Steuerreformen, in: Hansmeyer, K.H. (Hrsg.), Staatsfinanzierung im Wandel, Berlin, S. $189-211$

Folkers, C. (1983c) Konzepte zur Begrenzung von Steuern und Staatsausgaben, in: Arnim v., H.H., Littmann, K., (Hrsg.), Finanzpolitik im Umbruch: Zur Konsolidierung öffentlicher Haushalte, Berlin, S. $163-179$

Folkers, C. (1984) Fiskalische Begrenzungen, in: Das Wirtschaftsstudium 13 , S. $500-506$

Folkers, C. (1985) Steuerrevorminteressen und Steuervergünstigungen in positiver und institutioneller Perspektive, in: Jahrbuch für Sozialwissenschaft 36, S. $274-301$

Folkers, D. (1975) Monopolistische Preispolitik bei stochastischer Nachfrage, Göttingen

Forte, F., Peacock, A. (1981) Tax planning, tax analysis and tax policy, in: Peacock, A., Forte, F. (Eds.), Oxford, S. $3-28$

Frey, B.S. (1976) Taxation in Fiscal Exchange, a comment, in: Journal of Public Economics $6,5.31-35$

Frey, B.S. (1978) Politico-Economic Models and Cycles, in: Journal of Public Economics 9, S. $203-220$

Frey, B.S. (1980) Politische ökonomie und Wirtschaftspolitik, in: Das Wirtschaftsstudium 9, S. $529-534$

Frey, B.S. (1981) Theorie demokratischer Wirtschaftspolitik, München

Friedland, N., Maital, S., Rutenberg, A. (1978) A Simulation Study of Income Evasion, in: Journal of Public Economics 10 , S. $107-116$

Friedman, M. (1971) Kapitalismus und Freiheit, Stuttgart 
Fromm, G., Taubman, P. (1973) Public Economic Theory and Policy, New York u.a.o.

Fullerton, D. (1982) On the Possibility of an Inverse Relationship Between Tax Rates und Government Revenues, in: Journal of Public Economics 19, S. $3-22$

Gandenberger, 0. (1966) Die Verbrauchsbesteuerung eines Angebotsmonopolisten, dargestellt als Variante zur Theorie des bilateralen Monopols, in: Jahrbücher für Nationalökonomie und Statistik 179 , S. $1-24$

Gandenberger, 0. (1975/76) Zur Integration von Preistheorie und Verbrauchsteuer, in: Finanzarchiv NF 34, S. $405-423$

Geary, R.C. (1950/51) A Note on 'A Constant Utility Index of the cost of Living', in: Review of Economic Studies 17, S. $65-66$

Gerloff, W. (1926) Steuerwirtschaftslehre, in: Gerloff, W., Meisel, F. (Hrsg.), Handbuch der Finanzwissenschaft, 1. Aufl., Bd. I, Tübingen, S. $436-489$

Glaeser, R. (1964) Finanzpolitische Willensbildung in der Bundesrepublik Deutschland. Versuch einer Analyse der Grundstruktur und Problematik, Berlin

Gluch, E. (1983) Die nächste Krise schon in Sicht, in: Wirtschaftswoche 6, S. 74

Goetz, C. (1974) Fiscal illusion in state and local finance, in: Borcherding, T.E. (Ed.), Budgets und bureaucrats: The sources of public sector growth, Durham, S. $178-187$

Goetz, M.L. (1978) Tax Avoidance, Horizontal Equity and Tax Reform: A Proposed Synthesis, in: Southern Economic Journal 44 , S. $798-812$

Goetz, M.L. (1980) The Normative Bases of Tax Reform: A Constitutional Perspective, in: Samuels, W.J., Wade, L.L. (Eds.), Taxing and Spending Policy, Lexington, Mass., Toronto, S. $61-66$

Goldman, S.M., Uzawa, H. (1964) A Note on Separability in Demand Analysis, in: Econometrica 32 , S. $387-389$

Good, D. (1980) The Politics of Anticipation, Making Canadian Federal Tax Policy, Ottawa

Gordon, S.H. (1976) The New Constracterians, in: Journal of Political Economy 84 , S. $573-590$

Green, H.A. (1975) Two Models of Optimal Pricing and Taxation, in: 0xford Economic Papers 27, S. 352 - 382 
Green, J., Sheshinski, E. (1979) Approximating the Gains from Welfare Reforms, in: Journal of Public Economics 11 , S. $179-194$

Grossekettler, H. (1981) Der Brennan-Buchanan-Plan zur Eindämmung der Staatstätigkeit, oder: Brauchen wir eine neue Finanzwissenschaft?, in: Finanzarchiv NF 39, S. $495-508$

Grossmann, E. (1948) Gedanken in der Finanzpolitik in der reinen Demokratie, Bern

Guesnerie, R. (1977) On the Direction of a Tax Reform, in: Journal of Public Economics 7, S. $179-202$

Härtel, H.-H. (1984) Wohnbau - nützlich, aber nicht produktiv, in: Wirtschaftsdienst 64, S. $234-236$

Hahn, F.H. (1973) On Optimum Taxation, in: Journal of Economic Theory 7, S. $96-106$

Hall, R.E., Rabushka, A. (1983) Low Tax, Simple Tax, Flat Tax, New York

Haller, H. (1971) Die Steuern, Tübingen

Haller, H. (1973/74) Ideale und weniger ideale Bedingungen für eine Steuerreform, in: Finanzarchiv NF $32,5.21-34$

Haller, H. (1980) Rationale Steuersysteme und Bestimmungsgründe empirischer Steuerverfassungen, in: Andel, N., Haller, H., Neumark, F. (Hrsg.), Handbuch der Finanzwissenschaft, 3. Aufl., Bd. II, Tübingen, S. $173-201$

Harberger, A.C. (1979) Taxation and Welfare, Chicago, London

Harris, R.G., Mackinnon, J.G. (1979) Computing Optimal Tax Equilibria, in: Journal of Public Economics 11 , S. $197-212$

Harsanyi, J.C. (1955) Cardinal Welfare, Individualistic Ethics, and Interpersonal Comparisons of Utility, in: Journal of Political Economy 63, S. $309-321$

Hartle, D.G. (1976) A Theory of the Expenditure Budgetary Process, Toronto, Buffalo

Hatta, T. (1977) A Theory of Piecemeal Policy Recommendations, in: The Review of Economic Studies $44, \mathrm{~S}, 1-21$

Hausman, J.A. (1981) Exact Consumer's Surplus and Deadweight Loss, in: The American Economic Review 71, S. $662-676$

Havemann, R.H. (1965) Water Resource Investment and the Public Interest, Nashville, Tennessee 
Hayek V., F.A. (1971) Die Verfassung der Freiheit, Tübingen, Engl. Fassung: The Constitution of Liberty, Chicago 1960

Hayek v., F.A. (1981) Recht, Gesetzgebung und Freiheit, Band 2: Die Illusion der sozialen Gerechtigkeit, Landsberg am Lech, Engl. Fassung: Law, Legislation and Liberty, Vol. II: The Mirage of Social Justice, London, Henley

Hedtkamp, G. (1977) Lehrbuch der Finanzwissenschaft, Darmstadt

Heilmann, M. (1975) Die Umsatzsteuerreform in der Bundesrepublik Deutschland. Die Entstehung des Umsatzsteuergesetzes von 1967 (Nettosatzsteuer). Eine Fallstudie zur steuerpolitischen Willensbildung in einer parlamentarischen Demokratie, Bern, Frankfurt/M.

Henderson, J.M., Quandt, R.E. (1983) Mikroökonom ische Theorie, 5. Aufl., München

Herder-Dorneich, Ph. (1959) Politisches Modell zur Wirtschaftstheorie. Theorie der Bestimmungsaktoren finanzwirtschaftlicher Staatstätigkeit, Freiburg

Hettich, W. (1979) Henry Simons on Taxation and the Economic System, in: National Tax Journal $32, S .1-9$

Hettich, W., Winer, S. (1984) A Positive Model of Tax Structure, in: Journal of Public Economics $24, S .67-87$

Hicks, J.R. (1939) Value and Capital, London

Hicks, J.R. (1943) The Four Consumer's Surpluses, in: Review of Economic Studies 11 , S. $31-41$

Hirschman, A.0. (1980) Leidenschaften und Interessen, Frankfurt/Main

Hochman, H.M. (1984) Rule Change and Transitional Equity, in:

Hochman, H.M., Peterson, G.E. (Eds.), Redistribution

through Public Choice, New York, London, S. 320 - 341

Hochman, H.M., Rodgers, J.D. (1969) Pareto optimal redistribution, in: American Economic Review 59, S. $542-557$

Hochschulverband (1980) Mitteilungen des Hochschulverbandes, 28. Jahrgang, Heft 6, S. $287-289$

Hotelling, H. (1935) Demand functions with limited budgets, in: Econometrica 3, S. $66-78$

Hotelling, H. (1938) The General Welfare in Relation to Problems of Taxation and of Railway und Utility Rates, in:

Econometrica 6, S. $242-269$ 
Hyman, D., Pasour, E. (1973) Property Tax Differentials and Residental Rents in North Carolina, in: National Tax Journal 26 , S. $303-307$

Issing, 0. (1983) Alibi für Fehlverhalten, in: Wirtschaftswoche 26, S. $54-58$

Johanson, L. (1979) The Bargaining Society and the Inefficiency of Bargaining, in: Kyklos 32 , S. $497-522$

Kaldor, N. (1955) An Expenditure Tax, London

Kaufer, E. (1981) Theorie der öffentlichen Regulierung, München

Kay, J.A. (1980) The Deadweight Loss from a Tax System, in: Journal of Public Economics 13, S. $111-119$

Keene, K. (1982) What do we know about the Public's Attitude on Progressivity?, in: National Tax Journal 36, S. $371-376$

Kemp, M., Ng, Y.-K. (1976) On the Existence of Social Welfare Functions, Social Orderings and Social Decision Functions, in: Economica 43, S. $59-66$

King, T.A. (1977) Estimating Property Tax Capitalization: A Critical Comment, in: Journal of Political Economy 85 , S. $425-431$

Kirchhof, P. (1973) Besteuerungsgewalt und Grundgesetz, Frankfurt/M.

Koskela, E. (1983) A note on progression, penalty schemes and tax evasion, in: Journal of Public Economics 22, S. $127-132$

Krause-Junk, G., Oehsen V., J.H. (1981) Besteuerung, optimale, in: Albers, W. u.a. (Hrsg.), Handwörterbuch der Wirtschaftswissenschaft, Bd. 9, S. $706-.723$

Krueger, A.0. (1974) The Political Economy of the Rent-Seeking Society, in: American Economic Review 64, S. $291-303$

Kuttner, R. (1980) Revolt of the Haves. Tax Rebellion and Hard Times, New York

Ladd, H.F., Tideman, T.N. (Eds.) (1981) Tax and Expenditure Limitations, Washington D.C.

Lambsdorff Graf, 0. (1984) Bessere Kapitalausstattung hilft dem Arbeitsamt, in: Der Arbeitgeber 20, S.776 - 779

Lampert, H. (1982) Das Transfersystem in der Bundesrepublik Deutschland, Bericht über das Gutachten der TransferEnquête-Kommission, in: Finanzarchiv NF $40,5.475-504$ 
Langner, P. (1983) Zero-Base-Budgeting und Sunset Legislation, Baden-Baden

Lau, L.J. (1970) Duality and the Structure of Utility Functions, in: Journal of Economic Theory 1, S. $374-396$

Laux, H. (1975) Entscheidungskriterien bei Unsicherheit, in: Das Wirtschaftsstudium 4, S. $159-164$

Lipsey, R.G., Lancaster, K. $(1956 / 57)$ The General Theory of Second Best, in: Review of Economic Studies 24, S. 11-32

Little, I.M.D. (1951) Direct versus Indirect Taxes, in: Economic Journal 61 , S. $577-584$

Little, I.M.D. (1952) Social Choice and Individual Values, in: Journal of Political Economy 60 , S. $422-432$

Littmann, K. (1970) Ein Valet dem Leistungsfähigkeitsprinzip, in: Haller, H., u.a. (Hrsg.), Theorie und Praxis des finanzpolitischen Interventionismus, Tübingen, S. 113-154

Littmann, K. (1975) Definition und Entwicklung der Staatsquote, Göttingen

Littmann, K. (1977) Problemstellung und Methoden der heutigen Finanzwissenschaft, in: Andel, N. U.a. (Hrsg.), Handbuch der Finanzwissenschaft, 3. Aufl., Bd. I, Tübingen, S. $99-120$

Littmann, K. (1984) Nur politisch motiviert, in: Wirtschaftswoche 17, S. $70-73$

Mackay, R.J., Weaver, C.L. (1978) Monopoly Bureaus and Fiscal Outcomes: Deductive Models and Implications for Reform, in: Tullock, G., Wagner, R.E. (Eds.), Policy Analys is and Deductive Reasoning, Lexington, Mass., Toronto, S. $141-165$

Mackay, R.J., Weaver, C.L. (1981) Agenda control by budget maximizers in a multi-bureau setting, in: Public Choice 37 , S. $447-472$

Magnusson, G. (1969) Production under Risk, Uppsala

Mann, F.K. (1937) Steuerpolitische Ideale, Vergleichende Studie zur Geschichte der ökonomischen Meinung 1600 - 1935, Jena

Mann, H. (1982) Dualität, Konsumentenrente und Messung der Mehrbelastung, in: Diskussionsbeiträge aus dem Institut für Volkswirtschaftslehre der Universität Hohenheim 10 
Mann, H. (1983) Der Dualitätsansatz in Modellen der optimalen Steuertheorie, in: Diskussionsbeiträge aus dem Institut für Volkswirtschaftslehre der Universität Hohenheim 17

Marglin, S.A. (1967) Public Investment Criteria, London

Markowitz, H. (1970) Portfolio Selection: Efficient diversification of investments, 2. Aufl., New Haven, London

Marshall, A. (1961) Principles of Economics, New York

McCormick, R., Tollison, R. (1981) Politicians, Legislation and the Economy. An Inquiry into the Interest-Group Theory of Government, Boston, The Hague, London

McGuire, T.G. (1981) Budget-Maximation Governmental Agencies:

An Empirical Test, in: Public Choice 36, S. $313-322$

McGuire, T.G., Garn, H.A. (1969) The Integration of Equity and Efficiency Criteria in Public Project Selection, in: The Economic Journal 79, S. $883-893$

Mckean, R.N. (1964) Divergences between individual and total costs within government, in: The American Economic Review 54 , S. $243-257$

Mckelvey, R.D. (1976) Intrasitivities in multidimensional voting models and some implications for agenda control, in: Journal of Economic Theory 12, S. $472-482$

Kckelvey, R.D. (1979) General conditions for global intransitivities in formal voting models, in: Econometrica 47 , S. $1085-1112$

Mckenzie, L.W. (1957) Demand theory without a utility index, in: Review of Economic Studies 24, S. $185-189$

Mill, J.S. (1921) in: Ashley, W. (Ed.), Principles of political economy, London

Miner, R.E., Chalice, D.R. (1978) Tax Reforms and Legislative Behavior, in: Tullock, G., Wagner, R. (Eds.), Policy Analysis and Deductive Reasoning, Lexington, Mass., Toronto, S. $61-76$

Mirrlees, J.A. (1971) An exploration in the theory of optimum income taxation, in: Review of Economic Studies 38, S. $175-208$

Mirrlees, J.A. (1972) On Producer Taxation, in: Review of Economic Studies 39, S. $105-111$

Mirrlees, J.A. (1975) Optimal Commodity Taxation in a Two Class Economy, in: Journal of Public Economics $4,5.27-33$ 
Mirrlees, J.A. (1977) Labour supply behaviour and optimal taxes, in: Fiscal Policy and Labour Supply. Institute for Fiscal Studies, London

Mittelsteiner, K.-H., Schaumburg, H. (1977) Abgabenordnung 1977 , 2. Aufl., Köln

Molitor, B. (1984) Ärgernis in der Marktwirtschaft, in: Wirtschaftswoche 3, S. $117-121$

Mueller, D.C. (1979) Public Choice, Cambridge, u.a.o.

Mueller, D.C., Murrell, P. (1983) Interest Groups and the Size of Government, in: discussion papers IIMV/Strukturpolitik IIM/Industrial Policy aus dem Wissenschaftszentrum Berlin. IIM/IP - $83-1$, Berlin

Munk, K.J. (1978) Optimal Taxation and Pure Profit, in:

Scandinavian Journal of Economics, S. $1-19$

Munk, K.J. (1980) Optimal Taxation with Some Non-Taxable Commodities, in: Review of Economic Studies 47 , S. $755-765$

Musgrave, A. (1981) 'Unreal Assumptions' in Economic Theory, in: Kyklos 34 , S. $377-387$

Musgrave, R.A. (1959) Theory of Public Finance, New York

Musgrave, R.A. (1974) Finanztheorie, Tübingen

Musgrave, R.A. (1976) ET, OT und SBT, in: Journal of Public Economics 6, S. $3-16$

Musgrave, R.A. (1981a) Leviathan Cometh - or Does He?, in: Ladd, H., Tideman, N. (Eds.), Tax and Expenditure Limitations, Washington D.C., S. $77-120$

Musgrave, R.A. (1981b) Der gegenwärtige Stand der Theorie der Besteuerung, in: Finanzarchiv NF 39, S. $29-42$

Musgrave, R.A. (1983a) Public Finance, now and then, in: Finanzarchiv NF $41, S .1-13$

Musgrave, R.A. (1983b) über den 'richtigen' Umfang des öffentlichen Sektors und die überexpansionshypothese, in:

Koslowski, P., Kreuzer, P., Löw, R. (Hrsg.), Chancen und Grenzen des Sozialstaats, Tübingen, S. $90-116$

Musgrave, R.A., Musgrave, P.B. (1984) Public Finance in Theory and Practice, New York u.a.0.14th ed,

Musgrave, R.A., Musgrave, P.B., Kullmer, L. (1979) Die öffentlichen Finanzen in Theorie und Praxis, 2. Auf1., Bd. 2, Tübingen 
Musgrave, R.A., Shoup, C.S. (1959) Readings in the Economics of Taxation. London

Neck, R. (1983) Zur politischen ökonomie von Steuerreformen, in: Hansmeyer, K.-H. (Hrsg.), Staatsfinanzierung im Wandel, Berlin, S. $141-169$

Neumann v., J., Morgenstern, 0. (1947) Theory of Games and Econom ic Behavior, Princeton

Neumark, F. (1970) Grundsätze gerechter und ökonomisch rationaler Steuerpolitik, Tübingen

Neumark, F. (1971) Zur Problematik von Steuerreformen in unserer Zeit, in: Geiger v., W. U.a. (Hrsg.), Der wirtschaftende Staat, Theorie und Praxis, Festschrift für Theo Keller, Bern, Stuttgart

Neumark, F. (1981) Steuersenkungen oder Steuererhöhungen, in: Wirtschaftsdienst 8 , S. $382-388$

Neumark, F. (1982) überstürzte Änderungen, in: Wirtschaftswoche 43 , S. $156-165$

Neumark, F. (1983) Wo bleibt die Wende?, in: Wirtschaftswoche 39, S. $74-84$

Niskanen, W.A. (1971) Bureaucracy and Representative Government, Chicago - New York

$\mathrm{Ng}$, Y. - K. (1977) Towards a theory of third best, in: Public Finance 32, S. $1-15$

$\mathrm{Ng}$, Y.-K. (1982) Beyond Pareto Optimality: The Necessity of Interpersonal Cardinal Utilities in Distributional Judgements of Social Choice, in: Zeitschrift für Nationalökonomie 42 , S. $207-233$

Nozick, R. (1974) Anarchy, State and Utopia, 0xford

Oakland, W.H. (1979) Proposition 13 - Genesis and Consequences, in: National Tax Journal 32 , S. $387-409$

Oakland, W.H. (1981) Proposition 13: Genesis and Consequences, in: Kaufman, G.G., Rosen, K.T. (Eds.), The Property Tax Revolt, Cambridge, Mass., S. $31-63$

0ates, W. (1969) The effects of property taxes and local public spending on property values: an empirical study of tax capitalization and the Tiebout hypothesis, in: Journal of Political Economy 77 , S. 957 - 971 
0ates, W. (1973) The effects of property taxes and local public spending on property values: a reply and yet further results, in: Journal of Political Economy $81, \mathrm{~S} .1004-1008$

Oehsen V., J.H. (1982) Optimale Besteuerung, Frankfurt

Oehsen V., J.H. (1983) Zum allokativen Spielraum einer Steuerreform. Referat für das Symposium "Steuerreform in Theorie und Praxis" in Brixen vom 08. - 12.06. 1983

0lson, M. (1968) Die Logik des kollektiven Handelns: Kollektivgüter und die Theorie der Gruppen, Tübingen

$0 l$ son, M. (1982) The Rise and Decline of Nations, New Haven, London

Parks, R. (1969) Systems of Demand Equations: an Empirical Comparison of Alternative Financial Forms, in:

Econometrica 37 , S. $629-650$

Parks, R. (1976) An Impossibility Theorem for Fixed Preferences: A Dictatorial Bergson-Samuelson Welfare Function, in: Review of Economic Studies 43, S. $447-450$

Pawlowski, Z. (1971) Einführung in die mathematische Statistik, Berlin

Pazner, E., Sadka, E. (1981) Welfare Criteria for Tax Reforms, in: Journal of Public Economics 16, S. $113-122$

Peacock, A. (1978) The Economics of Bureaucracy, An Inside View, in: The Institute of Economic Affairs (Ed.), The Economics of Politics, Lancing, S. $117-127$

Pechman, J.A. (1977) Federal Tax Policy, Washington

Pfähler, W. (1978) Normative Theorie der fiskalischen Besteuerung, Frankfurt/M., Bern, Las Vegas

Pfohl, H.-Chr., Braun, G.E. (1981) Entscheidungstheorie - Normative und deskriptive Grundlage der Entscheidung, Landsberg am Lech

Phlips, L. (1974) Applied Consumption Analysis, Amsterdam, 0xford

Piduch, E.A. (1964) Finanzverfassung und Steuerreform, Köln

Pohmer, D. (1970) Leistungsfähigkeitsprinzip und Einkommensumverteilung, in: Haller, H. U.a. (Hrsg.), Theorie und Praxis des finanzolitischen Interventionismus, Tübingen, S. $135-167$

Polinsky, A., Shavell, S. (1982) Pigouvian Taxation with Administrative Cost, in: Journal of Public Economics 19, S. $385-394$ 
Pollakowski, H. (1973) The Effects of Property Taxes and Local Public Spending on Property Values: A Comment and Further Results, in: Journal of Political Economy 81 , S. $994-1003$

Posner, R. (1975) The Social Costs of Monopoly and Regulation, in: Journal of Political Economy 83, S. $807-827$

Preusker, U.K. (1979) Politiksteuerung durch allgemeine Wahlen. Untersucht anhand einer Fallstudie über das Zustandekommen des Einkommensteuerreformgesetzes vom 5. August 1974, Studien zur Politik Bd. 5, Köln u.a.0.

Rabushka, A., Ryan, P. (1982) The Tax Revolt, Stanford, California

Radian, A. (1979) On the Differences between the Political Economy on Introducing and Implementing Tax Reforms - Israel 1975 - 1978, in: Journal of Public Economics 11 , S. $261-271$

Ramsey, F.P. (1927) A Contribution to the Theory of Taxation, in: Economic Journal 37 , S. $47-61$

Rawls, J. (1971) A Theory of Justice, Cambridge, Mass.

Rawls, J. (1974) Concepts of Distributional Equity: Some Reasons for the Maximum Criterion, in: American Economic Review, Papers and Proceedings 64, S. $141-146$

Reese, T. (1980) The Politics of Taxation, Westport, London

Ricketts, M. (1981) Tax theory and tax policy, in: Peacock, A., Forte, F. (Eds.), The Political Economy of Taxation, oxford, S. $29-46$

Riedel, W. (1982) Die steuerpolitische Willensbildung bei der Körperschaftsteuerreform 1977, Frankfurt/M.

Ritschl, H. (1925) Theorie der Statswirtschaft und Besteuerung, Bonn - Leipzig

Romer, T., Rosenthal, H. (1979) Bureaucrats Versus Voters: On the Political Economy of Resource Allocation by Direct

Democracy, in: Quarterly Journal of Economics 93 , S. $563-587$

Roppel, U. (1979) ökonomische Theorie der Bürokratie: Beiträge zu einer Theorie des Angebotsverhaltens staatlicher Bürokratien in Demokratien, Freiburg i.B.

Roppel, U. (1982) Wettbewerbs-versus Monopolmodell des öffentlichen Sektors, in: Jahrbuch für Neue Politische ökonomie, Bd. 1, Tübingen, S. $178-212$ 
Rose, G. (1983) Betriebswirtschaftlich bedeutsame Eigenschaften des Steuersystems, in: Hansmeyer, K.-H. (Hrsg.), Staatsfinanzierung im Wandel, Berlin, S. $81-108$

Rose, M., Wenzel, H.D., Wiegard, W. (1981) 0ptimale Finanzpolitik, Stuttgart, New York

Rose, M., Wiegard, W. (1983) Zur optimalen Struktur öffentlicher Einnahmen unter Effizienz- und Distributionsaspekten, in: Pohmer, D. (Hrsg.), Zur optimalen Besteuerung, Berlin, S. $9-162$

Rosen, K.T. (1982) The Impact of Proposition 13 on House Prices in Northern California: A Test of the Interjurisdictional Capitalization Hypothesis, in: Journal of Political Economy 90, S. $191-200$

Roy, R. (1947) La distribution du revenue entre les divers biens, in: Econometrica 15, S. $205-255$

Sah, R.K. (1983) How Much Redistribution is Possible Through Commodity Taxes?, in: Journal of Public Economics 20 , S. $90-101$

Salinger, E. (1981) Betriebswirtschaftliche Entscheidungstheorie, München

Samuelson, P.A. (1947) Foundations of Economic Analysis, Cambridge, Mass.

Samuelson, P.A. (1947/48) Some Implications of Linearity, in: Review of Economic Studies 15, S. $88-90$

Samuelson, P.A. (1.954) The Pure Theory of Public Expenditure, in: Review of Economics and Statistics 36, S. $387-389$

Samuelson, P.A. (1958) Review of I.d.V. Graaf, Theoretical Welfare Economics, in: The Economic Journal 68, S. 540

Samuelson, P.A. (1967) Arrow's Mathematical Politics, in: Hook, S. (Ed.), Human Values and Economic Policy: A Symposium, New York, S. 41 - 51

Samuelson, P.A. (1970) The Fundamental Approximation Theorem of Portfolio Analysis in Terms of Means, Variances and Higher Moments, in: Review of Economic Studies 37, S. $537-542$

Sandmo, A. (1974) A Note on the Structure of 0ptimal Taxation, in: American Economic Review 64, S. $701-706$

Sandmo, A. (1976) optimal Taxation. An Introduction to the Literature, in: Journal of Public Economics 6, S. $37-54$ 
Schäfer, H. (1977) Kontrolle der öffentlichen Finanzwirtschaft, in: Andel, N., Haller, H., Neumark, F. (Hrsg.), Handbuch der Finanzwissenschaft, 3. Aufl., Bd. I, Tübingen, S. $519-550$

Schäffle, A. (1895) Die Steuern, Allgemeiner Teil, Leipzig

Schanz, G. (1896) Der Einkommensbegriff und die Einkommensteuergesetze, in: Finanzarchiv, Bd. XIII

Schmidt, K. (1980) Grundprobleme der Besteuerung, in: Andel, N., Haller, H., Neumark, F. (Hrsg.), Handbuch der Finanzwissenschaft, 3. Aufl., Bd. II, Tübingen, S. $120-171$

Schmölders, G. (1949) Um ein rationales Steuersystem, in: Finanzarchiv 11 , S. $479 \mathrm{ff}$.

Schmölders, G., Hansmeyer, K.-H. (1980) Allgemeine Steuerlehre, 5. Aufl., Berlin

Schmölders, G., Schelle, K. (1978) Der manipulierte Steuerzahler, in: Karl-Bräuer-Institut des Bundes der Steuerzahler 39

Schneeweiß, H. (1967) Entscheidungskriterien bei Risiko, Berlin, Heidelberg, New York

Schneeweiß, H. (1978) ökonometrie, 3. Aufl., Würzburg, Wien

Schneider, D. (1983) öffentliche Einnahmen und Unternehmensrisiko, in: Hansmeyer, K. - H. (Hrsg.), Statsfinanzierung im Wandel, Berlin, S. $63-79$

Seidel, C. (1984) Optimalbesteuerung bei alternativen sozialen Wohlfahrtsfunktionen, in: Bös, D., Rose, M., Seidl, Chr. (Hrsg.), Beiträge zur neueren Steuertheorie, Berlin u.a.o., S. $37-64$

Sen, A.K. (1970) Interpersonal Aggregation and Partial Comparability, in: Econometrica 38, S. 393 - 409

Sen, A.K. (1977) Social Choice Theory: A Reexamination, in: Econometrica 45 , S. $53-89$

Sen, A.K. (1979) Personal Utilities and Public Judgements: or What's Wrong with Welfare Economics, in: Economic Journal 89 , S. $537-558$

Shapiro, P., Sonstelie, J. (1982) Did Proposition 13 Slay Leviathan?, in: The American Economic Review 72,

S. $184-190$

Shoup, C.S. (1970) Tax Reform, in: Haller, H. U.a. (Hrsg.), Theorie und Praxis des finanzpolitischen Interventionismus, Tübingen, S. $245-252$ 
Silberberg, E. (1972) Duality and the Many Consumer's Surpluses, in: The American Economic Review 62, S. 942 - 952

Simons, H. (1938) Personal Income Taxation, On the Definition of Income as a Problem of Fiscal Policy, Chicago

Sinn, H.-W. (1980) ökonomische Entscheidungen bei Ungewissheit, Tübingen

Slemrod, J., Yitzhaki, S. (1983) On Choosing a Flat-Rate Income Tax System, in: National Tax Journal 36, S. $31-44$

Smith, A. (1776) An Inquiry into the Nature and Causes of the Wealth of Nations, in: Cambell, R., Skinner, A. (Eds.), oxford 1976

Sohmen, E. (1976) Allokationstheorie und Wirtschaftspolitik, Tüb ingen

Stein, R.M., Hamm, E., Freeman, P.K. (1983) An Analysis of Support for Tax Limitation Referanda, in: Public Choice 40 , S. $187-194$

Stern, K. (1980) Das Staatsrecht der Bundesrepublik Deutschland, Bd. II, München

Stern, N.H. (1976) On the Specification of Models of Optimum Income Taxation, in: Journal of Public Economics 6,

S. $123-162$

Stern, N.H. (1982) Optimum Taxation with Errors in Administration, in: Journal of Public Economics 17, S. $181-211$

Stiglitz, J.E. (1982) Utilitarism and Horizontal Equity, in: Journal of Public Eonomics 18, S. $1-33$

Stiglitz, J.E., Boskin, M.J. (1977) Impact of Recent Development in Public Finance Theory on Public Policy Decisions. Some Lessons from the New Public Finance, in: American Economic Review 67 , S. $295-302$

Stiglitz, J.E., Dasgupta, P. (1971) Differential Taxation, Public Goods and Economic Efficiency, in: Review of Economic Studies 38 , S. $151-174$

Stoltenberg, G. (1983) Interview mit der Wirtschaftswoche, in Wirtschaftswoche vom 23. Dezember 1983. Wiederabgedruckt in: Finanznachrichten des Bundesministerium der Finanzen 36/83, Bonn, 21. Dezember 1983, S. $2-5$

Stone, J.R. (1954) Linear Expenditure Systems and Demand Analysis: an Application to the Pattern of British Demand, in: Economic Journal 64, S. $511-527$ 
Stuart, C.E. (1981) Swedish Tax Rates, Labour Supply and Tax Revenues, in: Journal of Political Economy 89, S. $1020-1038$

Stubblebine, C. (1981) Senate Joint Resolution 58, A Balanced Budget and Tax Limitation Amendment to the Constitution of the United States, Manuscript, Conference on Constraining Federal Taxing, Hoover Institution, Stanford

Stützel, W. (1982) Die aktuelle Steuerpolitik im Spannungsfeld zwischen Wirtschafts- und Gesellschaftspolitik, Vortrag auf dem 36. Deutschen Betriebswirtschafter-Tag, Berlin, 27. September 1982

Subventionsberichte (siebter und neunter) (1979, 1983) Bundesminister der Finanzen (Hrsg.), Sachgebiet 707, Drucksache $8 / 3097$ vom 08.08.1979 und Drucksache 10/352 vom 06.09.1983, Bonn

Surrey, S.S. (1973) Pathways to Tax Reform, Cambridge, Mass.

Surrey, S.S., McDaniel, P.R. (1980) The Tax Expenditure Concept and the Legislative Process, in: Aaron, H., Boskin, M.J. (Eds.), The Economics of Taxation, Washington D.C., S. $123-144$

Tiebout, C.(1956) A pure theory of local expenditures, in: Journal of Political Economy 64 , S. $416-424$

Tipke, K. (1976) Die Steuergesetzgebung in der Bundesrepublik Deutschland aus der Sicht des Steuerrechtswissenschaftlers - Kritik und Verbesserungsvorschläge, in: Steuer und Wirtschaft 53 , S. $293-310$

Tirole, J., Guesnerie, R. (1981) Tax Reform from the Gradient Projection Viewpoint, in: Journal of Public Economics 15 , S. $275-279$ und $292-293$

Transfer-Enquête-Kommission (1979) Zur Einkommenslage der Rentner, Zwischenbericht der Kommission, veröffentlicht durch die Bundesregierung, Stuttgart u.a.0.

Transfer-Enquete-Komm ission (1981) Das Transfersystem in der Bundesrepublik Deutschland, veröffentlicht durch die Bundesregierung, Stuttgart u.a.0.

Tresch, R.W. (1981) Public finance: A normative theory, Plano, Texas, Georgetown, Ontario

Tsiang, S.L. (1972) "The Rational of Mean-Standard Debiation Analysis, Skewness Preference, and the Demand of Money", in: The American Economic Review 62, S. 354 - 371 
Tullock, G. (1975) The Transitional Gains Trap, in: The Bell Journal of Economics 6, S. $671-678$

Tullock, G. (1983) Economics of Income Redistribution, Boston, The Hague, London

Varian, H.R. (1981) Mikroökonomie, München, Wien

Varian, H.R. (1983) Non-parametric Tests of Consumer Behavior, in: Review of Economic Studies 1, S. $99-110$

Waffenschmidt, H. (1984) Entbürokratisierung als Schwerpunkt der Bundespolitik, Rede anläßlich des Fachkongresses "Neues Städtebaurecht" der Konrad-Adenauer-Stiftung am 17. Mai 1984, in: Bulletin des Presse- und Informationsamtes der Bundesregierung 58, S. 521 - 524

Wagner, A. (1890) Finanzwissenschaft, 2. Theil: Theorie der Besteuerung, Gebührenlehre und allgemeine Steuerlehre, Leipzig

Wagner, R.E. (1976) Revenue structure, fiscal illusion and budgetary choice, in: Public Choice $24-28$, S. $45-61$

Wagner, R.E. (1966) Pressure Groups and Political Entrepreneurs, in: Papers on Non-Market Decision Making, Charlottesville, Va., S. $161-170$

Wassermayer, F. (1983) Erfahrungen mit dem Außensteuergesetz von 1972, in: Hansmeyer, K.-H. (Hrsg.), Staatsfinanzierung im Wandel, Berlin, S. $573-591$

Weber, J. (1977) Die Interessengruppen im politischen System der Bundesrepublik Deutschland, Stuttgart u.a.o.

Weilepp, M. (1982) Subventionsabbau tut not - aber wie?, in: Wirtschaftsdienst 9 , S. $329-333$

Weisbrod, B.A. (1968) Income Redistribution Effects and BenefitCost Analysis, in: Chase, S. (Ed.), Problems in Public Expenditure Analysis, Washington D.C., S. $177-213$

West, E.G., Corke, G. (1980) Tax Constraints on Leviathan, in: Journal of Public Economics 13, S. $395-401$

Weymark, J.A. (1978) On Pareto-Improving Price Changes, in: Journal of Economic Theory 19, S. $338-346$

Weymark, J.A. (1979) A Reconciliation of Recent Results in Optimal Taxation Theory, in: Journal of Public Economics 12 , S. $171-189$

Weymark, J.A. (1980) Duality Results in Demand Theory, in: European Economic Review 14, S. $377-395$ 
Weymark, J.A. (1981) Undom inated directions of tax reform, in: Journal of Public Economics 16, S. 343-347 und $366-367$

Wicksell, K. (1896) Finanztheoretische Untersuchungen, Jena

Wiegard, W. (1976) Zur Theorie optimaler indirekter Steuern, in: Finanzarchiv NF 35, S. $195-217$

Wiegard, W. (1978) Optimale Schattenpreise und Produktionsprogramme für öffentliche Unternehmen. Second-Best-Modelle im finanzwirtschaftlichen Staatsbereich, Frankfurt am Main

Wiegard, W. (1980) Theoretische überlegungen zu einer schrittweisen Reform der indirekten Steuern, in: Jahrbuch für Sozialwissenschaft 31 , S. $1-20$

Wiegard, W. (1982) Erkenntnisgegenstand und -inhalt der "Neuen Finanztheorie", in: Finanzarchiv NF 40, S. $155-166$

Wildavsky, A. (1975) Budgeting, a comparative theory of budgetary process, Boston, Toronto

Wildavsky, A. (1980) How to Limit Government Spending, Berkeley, Los Angeles, London

Wildavsky, A. (1981) A Uniform Income Tax, in: Tax Notes 12, S. $611-612$

Windisch, R. (1980) Die Baumolsche "Kostenkrankheit" öffentlicher Dienste, in: Wirtschaftsstudium 11, S. $559-563$, u. 12, S. $613-615$

Woll, A. (1984) Wirtschaftspolitik, München

Zimmermann, H. (1977) Instrumente der Finanzpolitik, in:

Andel, N., Haller, H., Neumark, F. (Hrsg.), Handbuch der

Finanzwissenschaft, 3. Aufl., Band I, Tübingen, S. 165-192

Zumstein, P. (1977) Die Ausgabensteuer, Volkswirtschaftliche Begründung und praktische Durchführbarkeit, Diessenhofen 


\section{HOHENHEIMER VOLKSWIRTSCHAFTLICHE SCHRIFTEN}

Band 1 Walter Deffaa: Anonymisierte Befragungen mit zufallsverschlüsselten Antworten. Die Randomized-Response-Technik (RRT). Methodische Grundlagen, Modelle und Anwendungen. 1982.

Band 2 Thomas Michael Baum: Staatsverschuldung und Stabilisierungspolitik in der Demokratie. Zur neoinstitutionalistischen Kritik der keynesianischen Fiskalpolitik. 1982.

Band 3 Klaus Schröter: Die wettbewerbspolitische Behandlung der leitungsgebundenen Energiewirtschaft. Dargestellt am Beispiel der Fernwärmewirtschaft der Bundesrepublik Deutschland. 1986.

Band 4 Hugo Mann: Theorie und Politik der Steuerreform in der Demokratie. 1987. 
Hugo Mann - 978-3-631-75532-7

Downloaded from PubFactory at 01/11/2019 03:48:02AM

via free access 